\title{
On the Geometry of the Hamilton-Jacobi Equation
}

Miguel Vaquero Vallina
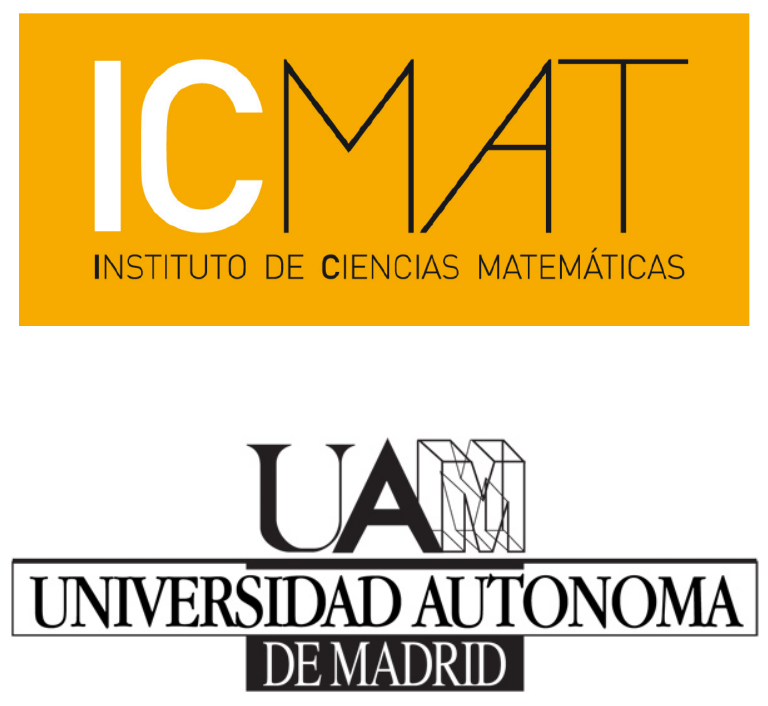

Madrid, 2015 



\title{
On the Geometry of the Hamilton-Jacobi Equation
}

\author{
Miguel Vaquero Vallina
}

Advisor: Manuel de León Rodríguez

Instituto de Ciencias Matemáticas, ICMAT

Universidad Autónoma de Madrid, UAM

Madrid, Spain 
Lector: Marco Zambon

Lector: Frans Cantrijn

Lector: Hernán Cendra

Tutor: Rafael Orive 


\section{Contents}

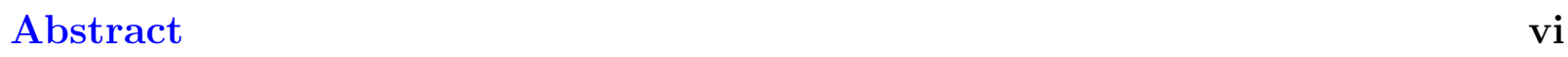

Resumen viii

Acknowledgements $\quad$ x

1 Introduction $\quad 1$

1.1 Historical Notes . . . . . . . . . . . . . . . . . . . . . . . . 1

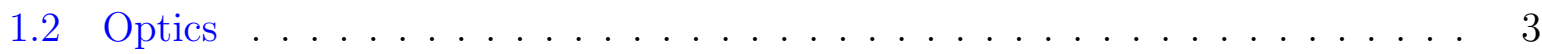

1.3 The Mechanical Analogy . . . . . . . . . . . . . . . . . . 4

1.4 Classical Mechanics and Symplectic Geometry . . . . . . . . . . . . . . 6

1.4.1 Hamiton-Jacobi Theory: Time-independent case . . . . . . . . . . . . 9

1.4.2 Hamilton-Jacobi Theory: Time-dependent case . . . . . . . . . . . . . 10

1.4.3 Hamilton-Jacobi Theory: Complete Solutions . . . . . . . . . . . . . . 11

1.4 .4 Generating Functions . . . . . . . . . . . . . . . . 13

1.5 Numerical Methods based on H-J Theory . . . . . . . . . . . . . . . . . . . 14

1.6 Topics related to H-J theory and Generating Functions . . . . . . . . . . . 15

$\begin{array}{ll}\text { Bibliography } & 17\end{array}$

Scientific articles presented in this dissertation 23

1.7 Hamiton-Jacobi theory, Symmetries and Coisotropic Reduction . . . . . . . 23

1.8 On the Geometry of Hamilton-Jacobi theory . . . . . . . . . . . . . . . . . . 24

1.9 A Hamilton-Jacobi theory on almost-Poisson Manifolds . . . . . . . . . . . . 25

1.10 Hamilton-Jacobi for Singular Lagrangians . . . . . . . . . . . . . . . . . . . . . . . . . . . . . . . . .

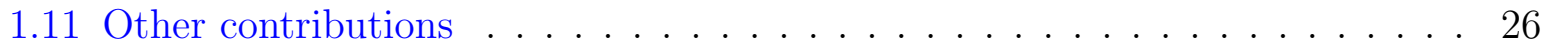

$\begin{array}{lr}\text { Conclusions and Future Work } & 187\end{array}$

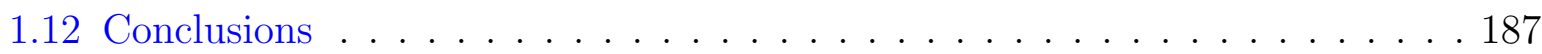

1.13 Future Work . . . . . . . . . . . . . . . . . . 188

Conclusiones y Trabajo Futuro 191

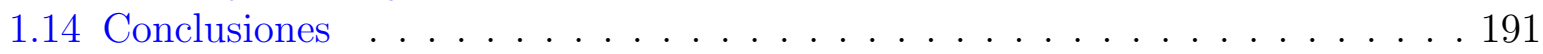

1.15 Trabajo Futuro . . . . . . . . . . . . . . . . 192 


\section{Abstract}

The classical Hamilton-Jacobi theory is well-understood from the symplectic geometry viewpoint. By Hamilton-Jacobi theory we mean the relation between certain PDE, the Hamilton-Jacobi equation, and Hamilton's equations, an ODE. These relations provide means to integrate the Hamilton's equations (or approximate them through canonical transformations). The main goal of this work is to extend the Hamilton-Jacobi theory to different geometric frameworks (reduction, Poisson, almost-Poisson, presymplectic...) and obtain new ways, analytic and numeric, to integrate Hamilton's equations in the corresponding geometric settings. Furthermore, one of the main points of this work is to develop a geometric setting where new numerical methods can be built on.

In Chapter 1 we sketch the historical development of the Hamilton-Jacobi theory. We briefly introduce some of the connections of the theory with optics and other analytical, geometrical and dynamical issues. In this chapter, we introduce several viewpoints and sketch their connections: analytic, geometric and dynamic. We emphasize the role of lagrangian submanifolds, because lagrangian submanifolds will be the keystone to achieve our goals.

In Chapter 2 we develop a reduction theory for the Hamilton-Jacobi equation. Reduction is one of the oldest and most useful techniques in geometric mechanics, so it is natural to wonder if that theory can be extended to the Hamilton-Jacobi theory, and take advantage of it. Ge and Marsden attempted to solve that question in [33]. We propose a new approach, general enough to include the previous ones and to give new insights to develop more applications.

In Chapter 3, based on symplectic groupoids, we construct a Hamilton-Jacobi theory for linear Poisson structures (duals of Lie algebroids). This framework is very interesting in order to integrate analytically and numerically Hamilton's equations, and it solves some previous questions of the area. We review and complete some previous works by Channell, Ge, Marsden, Scovel and Weinstein.

In Chapter 4 we present a Hamilton-Jacobi theory for almost-Poisson manifolds. The main objective is to understand from a purely geometric way the Hamilton-Jacobi theory for non-holonomic systems in [18, 40, 48].

Chapter 5 includes some extensions of the Hamilton-Jacobi theory, in order to deal with singular lagrangians. Singular lagrangians are common in classical field theory, and so understanding them in the classical mechanics context seems to be a natural step.

Finally, some conclusions and future work are analyzed in the last part of this thesis. 


\section{Resumen}

La teoría clásica de Hamilton-Jacobi es, hoy en día, bien conocida desde el punto de vista de la geometría simpléctica. A lo largo de esta memoria por teoría de Hamilton-Jacobi se entiende la relación entre cierta EDP, la ecuación de Hamilton-Jacobi, y las ecuaciones de Hamilton (EDO). Ello proporciona medios para integrar las ecuaciones de Hamilton, o aproximarlas a través de transformaciones canónicas. La meta principal de este trabajo es extender esa teoría a otros contextos (reducción, Poisson, almost-Poisson, presimpléctico...) y obtener nuevas formas, analíticas y numéricas, de integrar las ecuaciones de Hamilton en otros marcos geométricos. Más aún, uno de los puntos principales tratados aquí es el desarrollo de herramientas geométricas para la implementación de nuevos métodos numéricos.

En el Capítulo 1 esbozamos el desarrollo histórico de la teoría de Hamilton-Jacobi. Introducimos brevemente algunas de las conexiones de la teoría con la óptica y otros temas analíticos y dinámicos. Enfatizamos el papel de las subvariedades lagrangianas, porque dichas subvariedades serán la piedra angular para alcanzar nuestros objetivos.

En el Capítulo 2 presentamos una teoría de reducción de la ecuación de HamiltonJacobi. La teoría de reducción es una de las más antiguas y útiles técnicas de la mecánica geométrica, por lo que es muy natural preguntarse si dicha teoría puede ser combinada con la teoría de Hamilton-Jacobi y así obtener nuevos resultados basándose en ella. Ge y Marsden dieron los primeros pasos en esta dirección en [33]. Nosotros proponemos una nueva aproximación, suficientemente general como para incluir los resultados previos, pero al mismo tiempo damos una nueva visión y más aplicaciones.

En el Capítulo 3, usando grupoides simplécticos construimos una teoría de HamiltonJacobi para estructuras de Poisson linales (en el dual de un algebroide de Lie). Esta visión es muy interesante de cara a la integración analítica y numérica de las ecuaciones de Hamilton y resuelve algunas de las cuestiones del área. Revisamos y completamos trabajos previos de Channell, Ge, Marsden, Scovel y Weinstein.

En el Capítulo 4 presentamos una teoría de Hamilton-Jacobi para estructuras almostPoisson. La meta principal es entender desde un punto de vista enteramente geométrico la teoría de Hamilton-Jacobi para sistemas no-holónomos desarrollada en [18, 40, 48].

El Capítulo 5 incluye algunas extensiones de Hamilton-Jacobi para tratar con lagrangianos singulares. Los lagrangianos singulares son comunes en la teoría clásica de campos y por ello entenderlos en el ámbito de la mecánica clásica parece el primer paso a tomar.

Finalmente, discutimos las conclusiones y una perspectiva de trabajo futuro. 


\section{Agradecimientos}

Me gustaría empezar agradeciendo a mi director, Manuel de León. En primer lugar por darme la oportunidad de unirme a este proyecto, hace ya cuatro años . En segundo lugar por ser capaz de sacar siempre un rato para hablar de matemáticas, la vida académica y mi futuro, incluso cuando los innumerables quehaceres como director del ICMAT, miembro de IMU, ICSU (y otras tantas cosas y siglas que ahora no recuerdo) aprietan. Por último, quiero darle las gracias por enseñarme que cuando vienen turbulencias, hay que abrocharse el cinturón y seguir peleando. Quiero agradecer a David Martín de Diego, colaborador estrella y una de las mejores personas que he conocido, por su constante apoyo, sus enseñanzas (matemáticas y no matemáticas), por tener siempre la puerta abierta y diván preparado para él que lo necesite, y finalmente, por ser siempre capaz de crear el buen ambiente que lo caracteriza. Quiero agradecer a Daniel Peralta-Salas, porque desde aquellos tiempos en los que su despacho estaba al lado del mío, siempre ha tenido un rato para escuchar mis tonterías y mis preguntas triviales. Por darme una palmada en la espalda cuando la he necesitado y sobre todo, por compartir su amplia visión de las matemáticas y de su elaboración conmigo. Quiero agradecer a mis grandes amigos canarios, David Iglesias Ponte y Juan Carlos Marrero, ya que con ellos empecé a hacer investigación, porque gracias a ellos he desarrollado mi "guanche interior", y por su constante ayuda, ya sea discutiendo sobre algebroides o compartiendo unos momentos de relax acompañados de cerveza fria. Finalmente quiero agradecer a Alberto Enciso y Antonio Córdoba, aunque mi trato con ellos ha sido mucho menor, he aprendido mucho de sus comentarios y reflexiones durante cafés y comidas, o incluso encuentros fugaces en los pasillos, y a Manuel Pedreira Pérez y Modesto Salgado por haber sido los impulsores de que yo viniera al ICMAT a hacer mi doctorado, desde un Santiago que ya se me hace lejano.

De entre los post-doc que he conocido durante estos años, hay dos que han sido prácticamente hermanos mayores (collejas incluidas): Cédric M. Campos y Ángel Castro. Quiero agradecer a Cédric las innumerables veces que se ha preocupado por mi, que me ha ayudado y aconsejado. Por su energía y saber hacer. Por haberme hecho "marianico", motero, bailarín, cocinero, experto en Linux Mint..., en fin, sin él estos años habrían sido muchísimo más duros. Quiero agradecer a Ángel por sus continuas enseñanzas y reflexiones, su amistad sincera y sus ánimos interminables. También quiero agradecerle su guía en el mundo de la docencia. Me ha convencido de que los Rubio de Francia del 2013 son excepcionales.

Si en algo he tenido suerte, es en los amigos que me han tocado. Pero de entre todos ellos 
hay gente que lleva muchos años conmigo, que hacen que Pamplona, Alemania o Princeton esté cerca. Quiero destacar a Brais, Burky, Javi Gómez, Juan Carlos y Sandro. Por todo lo que he aprendido de ellos, porque a ellos recurro cuando las cosas van bien y cuando las cosas van mal. Quiero destacar también a Daniel Lear, David Fernández, Enrico, Irati, Jeza, María Barbero, Miguel Domínguez, Omar y Viviana, compañeros incansables de esta tesis y sobre todo, de esta etapa. Finalmente, Jano, Fani, Gonzalo, Graña, Isidro y Katín, que han estado conmigo desde "principio de los tiempos".

También envío un sincero agradecimiento a Mark Gotay y Rui Loja Fernandes, con quienes he realizado correspondientes estancias de doctorado en Vancouver y Urbana Champaign. Su trato ha sido más que bueno desde el primer momento y su puerta ha estado siempre abierta para todo lo que he necesitado. Espero seguir colaborando y aprendiendo de ellos muchos años.

No quiero olvidarme tampoco de Rafael Orive y Marco Zambon, tutor y lector de esta tesis. Así como Frans Cantrijn y Hernán Cendra, quienes han hecho una magnífica labor leyéndose esta memoria. Sebastián Ferraro, colaborador de uno de los pilares de esta tesis, ha detectado varios errores tipográficos y ha implementado los métodos numéricos tratados en esta disertación, contribuyendo significativamente a los resultados finales que aquí se exponen.

Por último, quiero agradecer y dedicar esta tesis a mi familia. Por su apoyo incondicional, su guía y su cariño. Pero sobre todo, porque entre mis recuerdos más felices están las vueltas del colegio con mi abuelo, las andainas con mi padre, los cafés con mi madre, y las charlas con mi hermana.

Sois todos lo que estáis, pero ni mucho menos estáis todos los que sois. En fin, a todos vosotros, que estáis aquí o que me he olvidado de nombrar,

iiiMUCHAS GRACIAS!!! 


\section{Chapter 1}

\section{Introduction}

The aim of this section is to introduce the reader into the Hamilton-Jacobi theory. We start by giving some brief historical review and introducing the classical Hamilton-Jacobi theory. The purpose is neither to be rigorous, neither to be complete, just to sketch some of the basic, classical ideas behind the Hamilton-Jacobi theory. Those ideas motivated the work done in this dissertation to a great extent. We introduce the time-independent and the time-dependent Hamilton-Jacobi equation and its complete solutions. We also give a brief introduction to numerical methods based on the Hamilton-Jacobi equation.

\subsection{Historical Notes}

The fundamentals of the Hamilton-Jacobi theory were developed by W.R. Hamilton (18051865 ) in the 1820's for problems in wave and geometric optics. His goal was to bring the theory of optics to the same state of perfection that Lagrange had brought dynamics some years before. Hamilton carried out one of the first studies of geometrical optics in an arbitrary medium with varying index of refraction. By the age of 22 his work on optics was complete, unifying the particle and wave concepts of light into one elegant, comprehensive theory. Following an analogy between rays and trajectories of a mechanical system, Hamilton extended his concepts to mechanics. In his essay On a General Method in Dynamics (1834), he reduced the study of the motions of all free systems of attracting or repelling points to the search and differentiation of one central relation, or characteristic function. The characteristic function is the optical path length of a ray, regarded as a function of initial and final positions and times of the ray in geometric optics. In his Second Essay on a General Method in Dynamics (1835), he derived both what we now call the Hamilton-Jacobi equation and Hamilton's canonical equations. With that theory a branch of classical variational calculus and analytical mechanics in which the task of finding extremals or the task of integrating a Hamiltonian system of equations, is reduced to the integration of a first-order partial differential equation, the Hamilton-Jacobi equation. Despite the fact that the integration of partial differential equations is usually more difficult than solving ordinary equations, the Hamilton-Jacobi theory proved to be a powerful tool 
in the study of problems of optics, mechanics and geometry.

With an emphasis on mechanics, Carl Gustav Jacob Jacobi (1804-1851) deepened Hamilton's formulation, by clarifying some mathematical issues, and developing significant applications. These contributions were given in a series of lectures in 1842 and 1843, which were not published until 1866. The theory embodies a wave-particle duality, which figured in the advent of the de Broglie-Schrödinger wave mechanics. Hamilton-Jacobi theory also played an important role in the development of the theory of first order partial differential equations and the calculus of variations, like the method of characteristics.

In a view broader than that of the original work, a solution of the Hamilton-Jacobi equation is the generator of a canonical transformation, a symplectic change of variables intended to simplify the equations of motion. In this direction, the most important result of the Hamilton-Jacobi theory is Jacobi's theorem, which states that a complete integral of the equation

$$
\frac{\partial S}{\partial t}\left(t, x^{i}, \alpha^{i}\right)+H\left(t, x, \frac{\partial S}{\partial x}\left(t, x^{i}, \alpha^{i}\right)\right)=0,
$$

i.e. the solution of this equation, which will depend on the parameters $\alpha=\left(\alpha^{1}, \ldots, \alpha^{n}\right)$, provided that

$$
\operatorname{det}\left(\frac{\partial^{2} S}{\partial x^{i} \partial \alpha^{j}}\right) \neq 0
$$

makes it possible to obtain the complete integral of the Hamiltonian system by the formulas

$$
\frac{\partial S}{\partial x^{i}}(t, x, \alpha)=p_{i}, \quad \frac{\partial S}{\partial \alpha^{i}}(t, x, \alpha)=\beta_{i}
$$

The application of Jacobi's theorem to the integration of Hamiltonian systems is usually based on the method of separation of variables in special coordinates. This is a very powerful tool. In this regard, we quote V.I. Arnol'd

"The technique of generating functions for canonical transformations, developed by Hamilton and Jacobi, is the most powerful method available for integrating the differential equations of dynamics."

-V.I. Arnol'd, [3], p. 233.

In the mechanics' framework, there are solutions of a type different from that of Hamilton, which determine not only orbits but also invariant tori in phase space on which the orbits lie. These solutions, which are known to exist only under special circumstances, are the subject of the celebrated work of Kolmogorov, Arnol'd, and Moser. Even approximate invariants, constructed by approximate solutions of the Hamilton-Jacobi equation, have implications for stability of motion over finite times. Various forms and generalizations of the Hamilton-Jacobi equation occur widely in contemporary applied mathematics, for instance in optimal control theory. 


\subsection{Optics}

As outlined in the previous section, the Hamilton-Jacobi theory was motivated by some previous works in optics. Moreover, the roots of geometric mechanics go back to optics. Let us quote D.D. Holm and V.I. Arnol'd in this regard:

"The ideas underlying geometric mechanics first emerged in the principles of optics formulated by Galileo, Descartes, Fermat and Huygens. These underlying ideas were developed in optics and particle mechanics by Newton, Euler, Lagrange and Hamilton, with added contributions from Gauss, Poisson, Jacobi, Riemann, Maxwell and Lie, for example, then later by Poincaré, Noether, Cartan and others. In many of these contributions, optics and mechanics held equal sway."

-D.D. Holm, [37], p. xvii.

"The fundamental notions of hamiltonian mechanics (momenta, the hamiltonian function $H$, the form $p \cdot d q-H \cdot d t$ and the Hamilton-Jacobi equations) arose by the transforming of several very simple and natural notions of geometric optics, guided by a particular variational principle that of Fermat, into general variational principles (and in particular into Hamilton's principle of stationary action, $\left.\delta \int L \cdot d t=0\right)$.

-V.I. Arnold, [3], p. 248.

Because of that, it is natural to start this work sketching some basic concepts from optics, in order to motivate the Hamilton-Jacobi theory. We would not be rigorous here.

\section{Some Basic Geometric Optics}

Fermat's and Huygens' principles are the starting point for geometric optics. Here we briefly introduce them following $[3,37,38]$. For the sake of simplicity we will assume that the medium is isotropic.

Definition 1 (Optical Length) The optical length of the path $q(t)$ from $q_{0}$ to $q_{1}$ is the time of propagation of light from $q_{0}$ to $q_{1}$.

Under our assumptions, i.e., we are dealing wight an isotopic medium the time of propagation of light through the path $q(s),\left(q:[a, b] \rightarrow \mathbb{R}^{3}\right)$, is

$$
\text { time }=\int_{[a, b]} n(q(s))\langle\dot{q}(s), \dot{q}(s)\rangle d s
$$

where $n(q)$ is the index of refraction of the medium at the spatial point $q \in \mathbb{R}^{3}$, and $\langle\cdot, \cdot\rangle$ is the standard scalar product in $\mathbb{R}^{3}$.

Fermat's Principle: Fermat's principle states that the path between two points taken by a ray of light leaves the optical length stationary under variations in a family of nearby paths (see [37]). 
Remark 1 Fermat's principle defines a light ray and provides an example that guided people in recognising the principles of geometric mechanics and the Hamilton-Jacobi equation. The mechanics analog of Fermat's principle is Hamilton's principle (see next section), which says that the trajectories followed by mechanical systems are extremals of the functional $\int L d t$.

Definition 2 (Wave Front) Let $t$ be a positive real number, we look at the set of all points $q$ to which light from a given point $q_{0}$ can travel in time less than or equal to $t$. The boundary of this set, $\Phi_{q_{0}}(t)$, is called the wave front of the point $q_{0}$ after time $t$ and consists of points to which light can travel in time $t$ and not faster, that is, in less time. This definition is taken from [3].

Huygens' Principle: Let $\Phi_{q_{0}}(t)$ be the wave front of the point $q_{0}$ after time $t$. For every point $q$ of this front, consider the wave front after time $s, \Phi_{q}(s)$ Then the wave front of the point $q_{0}$ after time $s+t, \Phi_{q_{0}}(s+t)$, will be the envelope of the fronts $\Phi_{q}(s), q \in \Phi_{q_{0}}(t)$.

Remark 2 One could use this idea to motivate the construction of new solutions of first order PDE's from families of solutions through envelopes in [26], p. 94.

Definition 3 (Characteristic Function) Fix a point $q_{0}$, the characteristic function is the function that associates to any point $q$, the optical length from $q_{0}$ to $q$. That is $S_{q_{0}}$ is the optical length from $q_{0}$ to $q$.

Remark 3 The level sets of the characteristic function are nothing other than the wave fronts.

\subsection{The Mechanical Analogy}

We return now to classical mechanics. Here the trajectories of motion are also extremals of a variational principle, and one can construct mechanics as the geometric optics of a many-dimensional space, as Hamilton did. We will not develop this construction in full detail here, but will only enumerate those optical concepts which led Hamilton to basic mechanical concepts.

In classical mechanics, one usually starts with generalized coordinates $\left(q^{i}\right)$, which give the state (position) of the system under study. From the differential geometry viewpoint, generalized coordinates are just local coordinates on the configuration manifold. From Newton's laws one can achieve Euler-Lagrange equation, using D'Alembert's principle, which is the dynamic analogue to the principle of virtual work for applied forces in a static system, see [3, 35]. The Euler-Lagrange equations are

$$
\frac{d}{d t}\left(\frac{\partial L}{\partial \dot{q}_{i}}\right)-\frac{\partial L}{\partial q_{i}}=0
$$


Euler-Lagrange equations determine the fate of the system given an initial position $\left(q_{i}\right)$ and velocity $\left(\dot{q}_{i}\right)$. These equations are also obtained considering a variational principle, Hamilton's principle. The aforementioned principle is the analogous of Fermat's.

Hamilton's principle: Given a path in the configuration space $\gamma=q:[a, b] \rightarrow Q$, it satisfies Hamilton's equations if and only if it is an extremal for the functional

$$
\gamma=q(t) \rightarrow \int_{\gamma} L \cdot d t,
$$

where variations are taken among all curves $q(t)$ which satisfy the fixed end-point condition.

The similarities between both variational principles should now be clear. In the same way, the Huygen's principle gives the Hamilton-Jacobi equation by analogy. We must require that the extremals going from the point $\left(q_{0}, t_{0}\right)$ do not intersect elsewhere. Assume that we are working on a "central field of extremals'. More precisely, we associate to every pair $\left(\dot{q}_{0}, t\right)$ a point $(q, t)$ which is the end of the extremal with initial condition $q(0)=q_{0}$, $\dot{q}(0)=\dot{q}_{0}$. We say that an extremal $\gamma$ is contained in a central field if that mapping is nondegenerate. Then, we can define

$$
S_{q_{0}, t_{0}}(q, t)=\int_{\gamma} L d t,
$$

and look at the differential of this function.

Proposition 1 The differential of the function $S$ defined above is

$$
d S=p_{i} \cdot d q^{i}+H \cdot d t,
$$

where $p_{i}=\frac{\partial L}{\partial \dot{q}^{i}}, H=p_{i} \cdot q^{i}-L$.

Proof: See [3], p. 254.

Since the aim of this section is just to, briefly, motivate the Hamilton-Jacobi equation from the optical-mechanics analogy, we will stop here and develop the symplectic viewpoint in the next section. The hamiltonian formalism is already apparent in the previous theorems. But before ending we would like to stress that this connection goes further, one could continue motivating a complete solution of the Hamilton-Jacobi equation from the characteristic function, allowing the subscript $S_{q_{0}}$ to vary. We also would like to quote E. Schrödinger, regarding the importance of this relations.

"Hamilton's variation principle can be shown to correspond to Fermat's Principle for a wave propagation in configuration space (q-space), and the HamiltonJacobi equation expresses Huygens Principle for this wave propagation. Unfortunately this powerful and momentous conception of Hamilton is deprived, in most modern reproductions, of its beautiful raiment as a superfluous accessory, in favour of a more colourless representation of the analytical correspondence."

-E. Schrödinger, [49], Quantisation as a problem of proper values. Part II. 


\subsection{Classical Mechanics and Symplectic Geometry}

Nowadays it is widely believed that symplectic geometry is the natural language to describe problems in classical mechanics. Symplectic geometry is also believed to be a link between analysis and geometry. For instance, the Fourier transform and the pseudo-differential operators can be understood in a symplectic-geometric fashion (see [39]). In this section we will interpret the Hamilton-Jacobi equation form the symplectic geometric viewpoint.

Recall that Hamilton's equations, for a hamiltonian $H\left(q^{i}, p_{i}\right)$, in the phase space $\left(q^{i}, p_{i}\right)$ are given by

$$
\begin{aligned}
& \frac{d q}{d t}(t)=\frac{\partial H}{\partial p}\left(q^{i}(t), p_{i}(t)\right), \\
& \frac{d p}{d t}(t)=-\frac{\partial H}{\partial q}\left(q^{i}(t), p_{i}(t)\right) .
\end{aligned}
$$

If we are working with a time-dependent hamiltonian function $H\left(t, q^{i}, p_{i}\right)$ then we need to use the extended-phase space $\left(t, q^{i}, p_{i}\right)$, but Hamilton's equations are the same

$$
\begin{aligned}
\frac{d q}{d t}(t) & =\frac{\partial H}{\partial p}\left(t, q^{i}(t), p_{i}(t)\right) \\
\frac{d p}{d t}(t) & =-\frac{\partial H}{\partial q}\left(t, q^{i}(t), p_{i}(t)\right) .
\end{aligned}
$$

Along this section we will replace the phase space by a manifold $M$, with symplectic structure $\Omega$, obtaining the symplectic manifold $(M, \Omega)$. The product manifold $\mathbb{R} \times M$ will play the role of the extended phase.

Definition 4 (Symplectic Manifold) A symplectic manifold is a smooth manifold, $M$, equipped with a closed non-degenerate differential 2-form, $\Omega$, called the symplectic form.

Example 1 (Cotangent Bundles and $\mathbb{R}^{2 n}$ ) 1 . Given a manifold $Q$, its cotangent bundle $T^{*} Q$ (with natural projection $\pi_{Q}: T^{*} Q \rightarrow Q$ ) has a canonical symplectic structure. In order to show that, we introduce the Liouville 1-form, $\Theta$. Given a tangent vector $X \in T_{\alpha_{q}} T^{*} Q$, we define

$$
\Theta(X)=\alpha_{q}\left(T \pi_{Q}(X)\right)
$$

An easy computation shows that $\Omega=-d \Theta$ is a symplectic form on $T^{*} Q$. The "odd" minus sign in the definition is a convention.

2. It is easy to see that if we consider on $\mathbb{R}^{2 n}$ the usual global coordinates $q^{i}$ for the "first $n$ coordinates" and $p_{i}$ for the "second $n$ coordinates", and the form $d q^{i} \wedge d p_{i}$, then $\left(\mathbb{R}^{2 n}, d q^{i} \wedge d p_{i}\right)$ is a symplectic manifold. Moreover, this is the local model for all symplectic manifolds, as it will be seen later on. 
Given a manifold $M$, a $k$-form $\alpha$ and a vector field $X$ over $M$ the contraction of the vector field $X$ and the form $\alpha$ is the $k-1$-form $i_{x} \alpha$ on $M$ defined by

$$
i_{X} \alpha\left(Y_{1}, \ldots, Y_{k-1}\right)=\alpha\left(X, Y_{1}, \ldots, Y_{k-1}\right),
$$

where $Y_{1}, \ldots, Y_{k-1}$ are also vector fields on $M$.

Definition 5 (Hamiltonian Vector Field) Given a real-valued function, $H$, that we will call hamiltonian, on the symplectic manifold $(M, \Omega)$, the corresponding hamiltonian vector field is defined as the unique vector field satisfying the equation

$$
i_{X_{H}} \Omega=d H .
$$

Definition 6 (Darboux Coordinates) Given a symplectic manifold $M, \Omega$, we call a local coordinate chart $\left(q^{i}, p_{i}\right)$ a Darboux coordinate chart if the symplectic form $\Omega$ reads on that chart

$$
d q^{i} \wedge d p_{i}
$$

where Einstein's summation holds.

Remark 4 1. Darboux coordinates always exist, due to a famous theorem named after Darboux.

2. In Darboux coordinates the integral curves of the hamiltonian vector field defined by (1.3) are the solutions of Hamilton's equations (1.1).

3. For cotangent bundles, (Example 1), natural bundle coordinates provide Darboux coordinates.

On the other hand, on the product manifold $\mathbb{R} \times M$ we can consider the form given by the pullback of $\Omega$ by the natural projection $p: \mathbb{R} \times M \rightarrow M$. Making some abuse of notation we will denote $p^{*} \Omega$ by $\Omega$ as well, when there is no room for confusion. With this constructions at hand, given a time-dependent hamiltonian $H: \mathbb{R} \times M \rightarrow \mathbb{R}$ we define the corresponding time dependent hamiltonian vector field by

$$
\frac{\partial}{\partial t}+X_{H}
$$

where $X_{H}$ is defined by

$$
i_{X_{H}} \Omega=d H-\frac{\partial H}{\partial t} \cdot d t .
$$

Remark 5 Considering coordinates on $\mathbb{R} \times M$ given by the usual global coordinates on $\mathbb{R}$ and Darboux coordinates on $M$, the integral curves of the time-dependent vector field defined by (1.4) correspond to curves verifying (time-dependent) Hamilton's equations (1.2). 
Remark 6 Although we are not going to deepen into them, there are other structures useful to define the hamiltonian vector fields. For instance, the cosymplectic structures. In Chapter 3 we will define analogous vector fields using Poisson structures.

The final ingredient to introduce the symplectic viewpoint of the Hamilton-Jacobi equation are lagrangian submanifolds.

Definition 7 (Lagrangian Submanifolds) Let $(M, \Omega)$ be a $2 n$-dimensional, symplectic manifold and $i: L \rightarrow M$ an immersed submanifold of dimension $n$ (half the dimension of $M)$. We say that $L$ is a lagrangian submanifold if $i^{\star} \Omega=0$, i.e., if the symplectic structure is equal to 0 when restricted to $L$.

Remark 7 Lagrangian submanifolds are some of the central objects in symplectic geometry. There is a very famous creed by A.D. Weinstein, one of the fathers of symplectic geometry, highlighting their importance

"Everything is a lagrangian submanifold."

A.D. Weinstein

Another, equivalent, definition of lagrangian submanifolds can be given through the concept of symplectic orthogonal.

Definition 8 (Symplectic Orthogonal) Given a submanifold, $i: N \rightarrow M$, of a symplectic manifold $(M, \Omega)$, at any point $p \in N$ the symplectic orthogonal at $p$ is the vector subspace given by $X \in T_{p} M$ such that $\Omega(X, Y)=0$ for all $Y \in T_{p} N$. We denote that space by $T_{p} N^{\perp}$.

Lemma 2 Let $(M, \Omega)$ be a $2 n$-dimensional, symplectic manifold and $L$ an $n$-dimensional immersed submanifold. Then $L$ is a lagrangian submanifold if and only if at every point $p \in L$ we have $T_{p} L=T_{p} L^{\perp}$.

We introduce now different instances of the Hamilton-Jacobi equation from the symplectic viewpoint. The main idea, shared by all the cases below, is that a lagrangian submanifold lives in a level set of a hamiltonian $H$, if and only if it is tangent to the associate hamiltonian vector field, $X_{H}$. The following lemmas are easy to prove.

Lemma 3 Let $(M, \Omega)$ be a symplectic manifold and $i: L \rightarrow M$ a lagrangian submanifold. If $H: M \rightarrow \mathbb{R}$ is a function (a hamiltonian), then $H$ is constant on $L$ if and only if $X_{H}$ is tangent to $L$, i.e., at any $p \in L$ we have $X_{H} \in T_{p} L$.

The proof is trivial, but due to the importance and applications of this result in this thesis, we reproduce it here.

Proof: If $L$ lives in a level set of $H$, that means that $d H \in\left(T_{p} L\right)^{\circ}$ for all $p \in L^{1}$. Then the equation $\Omega\left(X_{H}, Y\right)=d H(Y)$ applied to $Y \in T_{p} L$ for $p \in L$ and Lemma 2 gives the result.

\footnotetext{
$1 \circ$ denotes the annihilator of a vector subspace.
} 


\subsubsection{Hamiton-Jacobi Theory: Time-independent case}

In this subsection we explain the time-independent case of the Hamilton-Jacobi theory, relating PDE's and Hamilton's equations (ODE's). The abstract idea is quite easy to understand and will be repeatedly applied to the following constructions. We will consider the cotangent bundle case, although the theory can be applied locally to any symplectic manifold using Darboux coordinates.

From now on we will consider the cotangent bundle $T^{*} Q$, of an $n$-dimensional manifold $Q$, endowed with the canonical symplectic structure described in Example $1,\left(T^{*} Q, \Omega=\right.$ $-d \Theta)$. Let $H: T^{*} Q \rightarrow \mathbb{R}$ be a hamiltonian and $X_{H}$ the associated hamiltonian vector field. We recall that the cotangent bundle is also endowed with the natural projection $\pi_{Q}: T^{*} Q \rightarrow Q$, and that sections of this (vector) bundle are just 1-forms. Given one of those forms, say $\gamma: Q \rightarrow T^{*} Q$, then we can project the hamiltonian vector field $X_{H}$ through $\gamma$. We call the projected vector field through $\gamma$ and denote it by $X_{H}^{\gamma}$, the vector field on $Q$ defined by

$$
X_{H}^{\gamma}(q)=T \pi_{Q}\left(X_{H}(\gamma(q))\right) \text { for all } q \in Q .
$$

One obvious feature of the projected vector field is that it lives in $Q$, and that has half the dimension of $T^{*} Q$. Because of this reason, it is supposed to be easier to integrate. The Hamilton-Jacobi theory that we are going to introduce now relates the projected vector field, $X_{H}^{\gamma}$ and the hamiltonian vector field $X_{H}$. Before proceeding we introduce one more concept.

Definition 9 ( $f$-related Vector Fields) Consider $N, M$ differentiable manifolds and $f: N \rightarrow M$ a mapping between them. Let $X$ be a vector field on $N$ and $Y$ a vector field on $M$. Then, we say that the vector fields $X$ and $Y$ are $f$-related if for all $p \in N$

$$
T f(p)(X)=Y(f(p))
$$

holds.

Theorem 4 (Hamilton-Jacobi: Time Independent Case) Under the conditions above, if the form $\gamma$ is closed $(d \gamma=0)$ then the following conditions are equivalent:

1. The vector fields $X_{H}^{\gamma}$ and $X_{H}$ are $\gamma$-related.

2. The equation $d(H \circ \gamma)=0$ holds.

Remark 8 The proof is an immediate consequence of Lemma 3. See for instance [1] or [19].

Remark 9 Let us analyse the equation $d(H \circ \gamma)=0$ for a moment. Take $\left(q^{i}\right)$ coordinates on $Q$ and $\left(q^{i}, p_{i}\right)$ the corresponding natural coordinates in the cotangent bundle, $T^{*} Q$. Since the form $\gamma$ is closed, by Poincaré's Lemma (locally) there exists a function $S$, such that $d S=\gamma$. Then $d(H \circ \gamma)=0$ reads $d(H \circ d S)=0$ or assuming that we are in a connected component

$$
H\left(q^{i}, \frac{\partial S}{\partial q^{i}}\left(q^{i}\right)\right)=E,
$$

where $E \in \mathbb{R}$ is a constant. This is one of the usual forms of the Hamilton-Jacobi equation. 


\subsubsection{Hamilton-Jacobi Theory: Time-dependent case}

In the previous subsection we have introduced the equation $H\left(q^{i}, \frac{\partial S}{\partial q^{i}}\left(q^{i}\right)\right)=E$. But when one thinks about the Hamilton-Jacobi equation the expression

$$
\frac{\partial S}{\partial t}+H\left(t, q^{i}, \frac{\partial S}{\partial q^{i}}\left(t, q^{i}\right)\right)=0
$$

comes to mind. In this subsection we will obtain this equation in the same way that we have obtained equation (1.5). It will be seen that both expressions are consequences of the same abstract idea.

In order to achieve our goals, we need to introduce the extended-formalism. This formalism, provides a way to deal with the space $\mathbb{R} \times T^{*} Q$, introduced to deal with timedependent hamiltonians. The idea is quite simple, $\mathbb{R} \times T^{\star} Q$, cannot be a symplectic manifold because it is odd-dimensional. The solution is to carry the dynamics to $\left(\mathbb{R} \times T^{*} Q\right) \times \mathbb{R} \equiv$ $\mathbb{R}^{2} \times T^{\star} Q \equiv T^{\star} \mathbb{R} \times T^{\star} Q \equiv T^{*}(\mathbb{R} \times Q)$, which is a symplectic manifold, and where the introduction of the $\mathbb{R}$-factor is harmless. On the manifold $\mathbb{R} \times Q$ we consider coordinates given by the product of the global coordinate on $\mathbb{R}, t$, and coordinates $\left(q^{i}\right)$ on $Q$. The cotangent bundle, $T^{*}(\mathbb{R} \times Q)$, can be endowed with natural coordinates $\left(t, e, q^{i}, p_{i}\right)$, where $e$ is the $t$-conjugated momentum and $p_{i}$ is the momentum corresponding to $q^{i}$. There is a natural map

$$
\pi: T^{*}(\mathbb{R} \times Q) \rightarrow \mathbb{R} \times T^{*} Q
$$

given in local coordinates by $\pi\left(t, e, q^{i}, p_{i}\right)=\left(t, q^{i}, p_{i}\right)$.

Let $H$ be a time-dependent hamiltonian, so $H: \mathbb{R} \times T^{*} Q \rightarrow \mathbb{R}$. We define the extended hamiltonian as $H^{e}=\pi^{\star} H+e$, so the extended hamiltonian is a real valued function on $T^{*}(\mathbb{R} \times Q)$.

Remark 10 A trivial computation shows that the vector fields $X_{H^{e}}$ and $\frac{\partial}{\partial t}+X_{H}$ are $\pi$-related.

An application of Theorem 4 gives the following result.

Theorem 5 (Hamilton-Jacobi: time dependent case) Under the previous considerations, let $\gamma$ be a 1-form on $\mathbb{R} \times Q$. Then the following statements are equivalent:

1. The vector fields $X_{H^{e}}^{\gamma}$ and $\frac{\partial}{\partial t}+X_{H}$ are $\pi \circ \gamma$-related.

2. The expression $d\left(H^{e} \circ \gamma\right) \in\langle d t\rangle$ holds ${ }^{2}$.

Proof: It is a straightforward application of the time-independent case.

\footnotetext{
${ }^{2}$ The expression means that $d\left(H^{e} \circ \gamma\right)$ is in the differential ideal generated by $d t$, which just means that $d\left(H^{e} \circ \gamma\right)$ is equal to a real function on $\mathbb{R} \times Q$ times $d t$.
} 
Remark 11 Now equation $d\left(H^{e} \circ \gamma\right) \in\langle d t\rangle$ is more general than (1.6). Once more, using Poincaré's Lemma, we can locally write $\gamma=d S$ and write the previous expression in terms of $S$. As particular case, $d\left(H^{e} \circ d S\right) \in\langle d t\rangle$, includes the equations

$$
\begin{aligned}
& \frac{\partial S}{\partial t}+H\left(t, q^{i}, \frac{\partial S}{\partial q^{i}}\left(t, q^{i}\right)\right)=0, \\
& \frac{\partial S}{\partial t}+H\left(t, q^{i}, \frac{\partial S}{\partial q^{i}}\left(t, q^{i}\right)\right)=f(t),
\end{aligned}
$$

where $f(t)$ is an arbitrary real-valued real function. These equations are the HamiltonJacobi equations that one usually finds in the books.

Remark 12 If $H\left(q^{i}, p_{i}\right)$ is a time-independent hamiltonian, then equations can be related. Let $W$ be a solution for the time-independent Hamilton-Jacobi equation (1.5), then $S=$ $E \cdot t+W$ is a solution of the time-dependent Hamilton-Jacobi equation (1.6). Usually the function $W$ is called the characteristic function and $S$ the principal function (see [35]). We will show how to interpret these relations geometrically in the fist article of this dissertation, [22].

\subsubsection{Hamilton-Jacobi Theory: Complete Solutions}

This aspect of the Hamilton-Jacobi theory is maybe the most important one, at least, from our viewpoint. A complete solution of the Hamilton-Jacobi equation is a family of solutions depending on enough parameters. A complete solution allows to completely integrate Hamilton's equations, under the assumption of some regularity conditions. After discussing in some detail these complete solutions we also introduce the theory of generating functions. We briefly describe here the local aspects of these theories, the global counterpart is explained in the paper "On the Geometry of the Hamilton-Jacobi equation and Generating Functions", Chapter 3 of this thesis.

\section{Classical (local) theory:}

Due to its importance here, we sketch in this subsection the classical results. They are local and written in a coordinate dependent way, but we think that the global, geometric aspects of the theory are easier to understand after taking a look at the classical theory. We follow $[3,35]$.

Let $H\left(t, q^{i}, p_{i}\right)$ be a hamiltonian on the phase space $\left(t, q^{i}, p_{i}\right)$. By complete solution of the Hamilton-Jacobi equation for $H$, we mean the following

Definition $10 A$ complete solution of the Hamilton-Jacobi equation for the hamiltonian $H\left(t, q^{i}, p_{i}\right), i=1, \ldots, n$ is a real-valued function $S\left(t, q^{i}, \alpha^{i}\right), i=1, \ldots, n$, such that

1. For every (fixed) value of the parameters $\alpha^{i}, S\left(t, q^{i}, \alpha^{i}\right)$ satisfies the Hamilton-Jacobi equation (1.6),

$$
\frac{\partial S}{\partial t}+H\left(t, q^{i}, \frac{\partial S}{\partial q^{i}}\left(t, q^{i}\right)\right)=0 .
$$


2. The non-degeneracy condition: consider the matrix with component $(i, j)$ given by $\frac{\partial^{2} S}{\partial q^{i} \partial \alpha^{j}}$, that we denote by $\left(\frac{\partial^{2} S}{\partial q^{i} \partial \alpha^{j}}\right)$, then $\operatorname{det}\left(\frac{\partial^{2} S}{\partial q^{i} \partial \alpha^{j}}\right) \neq 0$

Then we can define (at least locally by the implicit function Theorem) the following implicit, time-dependent transformation, from the $\left(t, q^{i}, p_{i}\right)$-space to the $\left(t, \alpha^{i}, \beta_{i}\right)$-space.

$$
\frac{\partial S}{\partial q^{i}}\left(t, q^{i}, \alpha^{i}\right)=p_{i}, \quad-\frac{\partial S}{\partial \alpha^{i}}\left(t, q^{i}, \alpha^{i}\right)=\beta_{i} .
$$

A computation shows that this transformation sends the system to equilibrium, i.e., Hamilton's equations (1.2) become now,

$$
\begin{aligned}
& \frac{d \alpha^{i}}{d t}(t)=0, \\
& \frac{d \beta_{i}}{d t}(t)=0 .
\end{aligned}
$$

see $[1,3]$

Let us call $\hat{S}$ the transformation induced by $S$ through $(1.7)\left(\operatorname{so} \hat{S}:\left(t, q^{i}, p_{i}\right) \rightarrow\left(t, \alpha^{i}, \beta_{i}\right)\right)$. Through the procedure that we are going to describe now, Hamilton's equations can be integrated. Given an initial condition for Hamilton's equations $\left(t_{0}, q_{0}^{i}, p_{i}^{0}\right)$, apply $\hat{S}$, say $\hat{S}\left(t_{0}, q_{0}^{i}, p_{i}^{0}\right)=\left(t_{0}, \alpha_{0}^{i}, \beta_{i}^{0}\right)$. Now Hamilton's equations become trivial, equation (1.8). Then a solution is given by $c: t \rightarrow\left(t_{0}+t, \alpha_{0}^{i}, \beta_{i}^{0}\right)$ and thereby, $\hat{S}^{-1} \circ c$ is a solution of Hamilton's equations (1.2). Of course, in this procedure we are assuming that $\hat{S}$ can be inverted explicitly, which is usually a non-trivial matter. A detailed proof can be seen in $[1,3,35,37]$

\section{Global (symplectic) Theory}

The global, geometric theory associated to the the procedure above is described in [29], which corresponds to Chapter 3 of this thesis. The main idea, is that we are looking for a canonical transformation, because the flow of Hamilton's equations is a canonical transformation for any time $t$, see proposition below.

Definition 11 (Symplectic transformation) If $\left(N, \Omega_{1}\right)$ and $\left(M, \Omega_{2}\right)$ are symplectic manifolds, a mapping $f: N \rightarrow M$ is symplectic or canonical if $f^{*} \Omega_{2}=\Omega_{1}$.

Proposition 6 (Liouville's Theorem) Let $(M, \Omega)$ be a symplectic manifold, and let $H: \mathbb{R} \times M \rightarrow \mathbb{R}$ be a Hamiltonian. Assume that the flow is given by $\phi: \mathbb{R} \times M \rightarrow M$ and $\phi_{t}(p)=\phi(t, m)$ for $p \in M$. Then, if the flow is defined for $t$, the mapping $\phi_{t}$ is symplectic, that is, $\phi_{t}^{*} \Omega=\Omega$.

Locally, canonical transformations are encoded in functions, the generating functions, and a computation shows that the generating functions of the flow satisfy the HamiltonJacobi equation. Instead of starting with a symplectic transformation and compute its generating function, we can go the other way around and start with a function $S\left(t, q^{i}, \alpha^{i}\right)$ and induce a symplectic mapping by (1.7). If we look what condition has to satisfy $S$ to give us the flow, we obtain the Hamilton-Jacobi equation. 


\subsubsection{Generating Functions}

The theory of generating functions is related to Hamilton-Jacobi equation, as we said above. Moreover, complete solutions of the Hamilton-Jacobi equation (Section 1.4.3) described above can be considered as a particular case of the generating function theory. A complete solution of the Hamilton-Jacobi equation is just the generating function which gives the flow of Hamilton's equations (1.2).

We motivate the forthcoming constructions through the following observations. Let $(M, \Omega)$ be a symplectic manifold and $f: M \rightarrow M$ a symplectic transformation (also called canonical map). The following proposition is central in sympletic geometry.

Proposition 7 The graph of a symplectic mapping $f$, graph $(f)$, is a lagrangian submanifold of the symplectic manifold $(M \times M, \Omega \ominus \Omega)^{3}$.

Assume now that $f\left(\mathbf{p}_{1}\right)=\mathbf{p}_{2}, \mathbf{p}_{i} \in M, i=1$, 2. If $\left(q^{i}, p_{i}\right)$ and $\left(\alpha^{i}, \beta_{i}\right)$ are Darboux coordinates around $\mathbf{p}_{1}$ and $\mathbf{p}_{2}$ respectively, and so $\left(q^{i}, p_{i}, \alpha^{i}, \beta_{i}\right)$ are (except for a sign) Darboux coordinates on $M \times M$. Let $\pi\left(q^{i}, p_{i}, \alpha^{i}, \beta_{i}\right)=\left(q^{i}, \alpha^{i}\right)$ (for the time being, this mapping is only locally defined) and assume that $\pi_{\mid \operatorname{graph}(f)}$ is a diffeomorphis, at least around $\mathbf{p}_{1}$ (from $\operatorname{graph}(f)$ to $\left.\left(q^{i}, \alpha^{i}\right)\right)$. Then, in the coordinates $\left(q^{i}, p_{i}, \alpha^{i}, \beta_{i}\right)$ there exist a function $S\left(q^{i}, \alpha^{i}\right)$, such that $\operatorname{graph}(f)$ is given by the relations

$$
\operatorname{graph}(f)=\left(q^{i}, \frac{\partial S}{\partial q^{i}}\left(q^{i}, \alpha^{i}\right), \alpha^{i},-\frac{\partial S}{\partial \alpha^{i}}\left(q^{i}, \alpha^{i}\right)\right) .
$$

These results are just a consequence in local coordinates of the lemma below.

Lemma 8 Let $Q$ be a differentiable manifolds and $\left(T^{*} Q, \Omega\right)$ its cotangent bundle with its canonical symplectic structure. Let $i: L \rightarrow T^{*} Q$ be a lagrangian submanifold such that $\pi_{Q}$ restricted to $L$ is a diffeomorphism from $L$ to $Q$, then $L=\operatorname{Im}(\gamma)$ where $\gamma$ is a closed 1 -form.

Remark 13 By Poincaré's Lemma, locally $\gamma=d S$ which gives the function mentioned before.

We could say that generating functions are real-valued functions which specify canonical transformations through its partial derivatives.

Remark 14 We assumed that $\pi_{\mid \operatorname{graph}(f)}$ is a (local) diffeomorphism, that is, we can "project" the lagrangian submanifold $\operatorname{graph}(f)$ onto the $\left(q^{i}, \alpha^{i}\right)$-space. There is a result (see [3], p. 268 ) which says that one can always chose $n$ coordinates among the $\left(\alpha^{i}, \beta_{i}\right)$, say $\left(\alpha^{l}, \beta^{k}\right)$ such that $\operatorname{graph}(f)$ projects onto the $\left(q^{i}, \alpha^{l}, \beta^{k}\right)$-space. The election of one or other space corresponds to the classical free and non-free generating functions, which will be explained in the paper "On the Geometry of the Hamilton-Jacobi equation", included in this thesis. For instance, if the lagrangian submanifold $\operatorname{graph}(f)$ is projected onto the $\left(q^{i}, \beta_{i}\right)$-space, the resulting generating function $S\left(q^{i}, \beta_{i}\right)$ is called a generating function of type 2 .

\footnotetext{
${ }^{3}$ If $\pi_{i}: M \times M \rightarrow M$ is the projection over th $i$-factor, then $\Omega \ominus \Omega=\pi_{1}^{*} \Omega-\pi_{2}^{*} \Omega$.
} 
One of the main points of symplectic transformations is that Hamilton's equations transform under that change into Hamilton's equations for a different hamiltonian. One could take advantage of this to simplify Hamilton's equations. Instead of starting with a symplectic transformation and computing its generating function we can provide a function (a time-dependent generating function) $S\left(t, q^{i}, \alpha^{i}\right)$ and study the canonical transformation given by

$$
\frac{\partial S}{\partial q^{i}}\left(t, q^{i}, \alpha^{i}\right)=p_{i}, \quad \frac{\partial S}{\partial \alpha^{i}}\left(t, q^{i}, \alpha^{i}\right)=\beta_{i} .
$$

If we have a Hamiltonian $H\left(t, q^{i}, p_{i}\right)$, then the mapping defined above transforms Hamilton's equations (1.2) into Hamilton's equations for a new hamiltonian $K$ obtained bye

$$
\frac{\partial S}{\partial t}+H\left(t, q^{i}, \frac{\partial S}{\partial q^{i}}\right)=K
$$

If, for example, the new hamiltonian $K$ turns out to depend only on the $\alpha^{i}$ coordinates, $K\left(t, \alpha^{i}\right)$, Hamilton's equations become trivially integrable.

$$
\begin{aligned}
& \frac{d \alpha^{i}}{d t}(t)=0 \\
& \frac{d \beta_{i}}{d t}(t)=-\frac{\partial K}{\partial \alpha^{i}} .
\end{aligned}
$$

Once more, all this results are interpreted in this thesis in a neat way, in Chapter 3. Again, lagrangian submanifolds play a fundamental role.

\subsection{Numerical Methods based on H-J Theory}

The development of a geometric structure to provide new numerical methods which conserve Poisson structures is one of the main achievements of this thesis. So we believe that it is worth to shortly introduce their predecessors, the symplectic integrators based on the Hamilton-Jacobi equation.

The Hamilton-Jacobi equation plays an important role in the development of numerical integrators that preserve the symplectic structure. There are numerous examples illustrating the superior preservation of phase-space structures and qualitative dynamics by symplectic integrators. A philosophy has emerged of attempting to preserve as much geometric structure as possible in numerical treatments [27, 36, 42]. 
"We enter here the second heaven of Hamiltonian theory, the realm of partial differential equations and generating functions. The starting point of this theory was the discovery of Hamilton that the motion of the system is completely described by a characteristic function $S$, and that $S$ is the solution of a partial differential equation, now called the Hamilton-Jacobi differential equation."

- E. Hairer, E. Lubich and G. Wanner [36], p. 197.

By the constructions in the previous sections, it should be clear that there is a correspondence, at least locally, between generating functions and symplectic mappings. The basic idea of geometric integrators based on the Hamilton-Jacobi theory is to approximate a complete solution of the Hamilton-Jacobi equation. Although the solution is only approximate, it will always provide a symplectic transformation through equation (1.7). The fact that one is approximating a hamiltonian flow through canonical transformation makes a big difference between geometric integrators and other algorithms. For instance, attractors can not appear. The natural question is

If symplectic methods based on Hamilton-Jacobi equation are so powerful, can we extend those ideas to the Poisson framework?

That is one of the main questions that we address in this thesis, with a positive answer, at least for the cases of interest.

\subsection{Topics related to H-J theory and Generating Func- tions}

The main goal of this chapter, was to motivate and introduce some of the classical results about the Hamilton-Jacobi theory. Nowadays, Hamilton-Jacobi theory is a vast theory with very different applications to a huge variety of settings. We would like to end this section mentioning some of them and including some remarks.

1. From the PDE's viewpoint, Hamilton-Jacobi equation and Hamiton's equations are related by the method of characterists. A general method to relate PDE's and ODE's. In general, there is a lack of solutions of the Hamilton-Jacobi equation; motivated by this fact P.L. Lions and M.G. Grandall, (see [15]) introduced the called viscosity solutions.

2. Viscosity solutions Hamilton-Jacobi equations are related to Weak KAM theory (Aubry and Mather), so there is beautiful relation between analysis and dynamical systems (see [24, 25]).

3. The called variational solution of the Hamilton-Jacobi equation, introduced by Chaperon ([13]), are also very interesting. They coincide with viscosity solutions for convex hamiltonians. 
4. Hamilton-Jacobi equation is related to quantization (see $[1,6]$ ). Moreover, HamiltonJacobi plays a fundamental role between optics, quantum mechanics and PDE.

5. In optimal control theory the Hamilton-Jacobi-Bellman theory is of fundamental importance. The HJB method can be generalized to stochastic systems as well. For instance, see [26], p. 594.

6. Important discoveries in symplectic topology are related to Hamilton-Jacobi theory and generating functions $[54,55]$. For instance, Viterbo thinks about the action functional as a generating function on an infinite-dimensional vector bundle. That interpretation allows him to conclude very interesting results. 


\section{Bibliography}

[1] Abraham, R., And Marsden, J. E. Foundations of mechanics. Benjamin/Cummings Publishing Co., Inc., Advanced Book Program, Reading, Mass., 1978. Second edition, revised and enlarged, With the assistance of Tudor Raţiu and Richard Cushman.

[2] Abraham, R., Marsden, J. E., And Ratiu, T. Manifolds, tensor analysis, and applications, second ed., vol. 75 of Applied Mathematical Sciences. Springer-Verlag, New York, 1988.

[3] Arnol'D, V. I. Mathematical methods of classical mechanics, vol. 60 of Graduate Texts in Mathematics. Springer-Verlag, New York, 1993. Translated from the 1974 Russian original by K. Vogtmann and A. Weinstein, Corrected reprint of the second (1989) edition.

[4] Barbero-Liñán, M., de León, M., Martín de Diego, D., Marrero, J. C., And MuÑOZ-LeCAndA, M. C. Kinematic reduction and the Hamilton-Jacobi equation. J. Geom. Mech. 4, 3 (2012), 207-237.

[5] Bates, S., Fasso, F., And Sansonetto, N. The Hamilton-Jacobi equation, integrability and nonholonomic systems. J. Geom. Mech. 6, 4 (2014), 441-449.

[6] Bates, S., And Weinstein, A. Lectures on the geometry of quantization, vol. 8 of Berkeley Mathematics Lecture Notes. American Mathematical Society, Providence, RI; Berkeley Center for Pure and Applied Mathematics, Berkeley, CA, 1997.

[7] Benzel, S., Ge, Z., And Scovel, C. Elementary construction of higher-order Lie-Poisson integrators. Phys. Lett. A 174, 3 (1993), 229-232.

[8] Born, M., And Wolf, E. Principles of optics: Electromagnetic theory of propagation, interference and diffraction of light. With contributions by A. B. Bhatia, P. C. Clemmow, D. Gabor, A. R. Stokes, A. M. Taylor, P. A. Wayman and W. L. Wilcock. Third revised edition. Pergamon Press, Oxford-New York-Paris, 1965.

[9] Cariñena, J. F., Gràcia, X., Marmo, G., Martínez, E., Muñoz-Lecanda, M. G., And Román-Roy, N. Geometric Hamilton-Jacobi theory for nonholonomic dynamical systems. Int. J. Geom. Methods Mod. Phys. 7, 3 (2010), 431-454. 
[10] Cendra, H., Marsden, J. E., and Ratiu, T. S. Lagrangian reduction by stages. Mem. Amer. Math. Soc. 152, 722 (2001), x+108.

[11] Channell, P. J., And Scovel, C. Symplectic integration of Hamiltonian systems. Nonlinearity 3, 2 (1990), 231-259.

[12] Channell, P. J., And Scovel, J. C. Integrators for Lie-Poisson dynamical systems. Phys. D 50, 1 (1991), 80-88.

[13] Chaperon, M. On generating families. In The Floer memorial volume, vol. 133 of Progr. Math. Birkhäuser, Basel, 1995, pp. 283-296.

[14] Coste, A., Dazord, P., And Weinstein, A. Groupoïdes symplectiques. In Publications du Département de Mathématiques. Nouvelle Série. A, Vol. 2, vol. 87 of Publ. Dép. Math. Nouvelle Sér. A. Univ. Claude-Bernard, Lyon, 1987, pp. i-ii, 1-62.

[15] Crandall, M. G., And Lions, P.-L. Viscosity solutions of Hamilton-Jacobi equations. Trans. Amer. Math. Soc. 277, 1 (1983), 1-42.

[16] de León, M., Marrero, J. C., And de Diego, D. M. A geometric HamiltonJacobi theory for classical field theories. In Variations, geometry and physics. Nova Sci. Publ., New York, 2009, pp. 129-140.

[17] de León, M., Marrero, J. C., and Martín de Diego, D. Preface: New trends in the Hamilton-Jacobi theory. J. Geom. Mech. 1, 3 (2009), i.

[18] de León, M., Marrero, J. C., And Martín de Diego, D. Linear almost Poisson structures and Hamilton-Jacobi equation. Applications to nonholonomic mechanics. J. Geom. Mech. 2, 2 (2010), 159-198.

[19] de León, M., Marrero, J. C., Martín de Diego, D., and Vaquero, M. On the Hamilton-Jacobi theory for singular Lagrangian systems. J. Math. Phys. 54, 3 (2013), 032902, 32 .

[20] de León, M., Martín de Diego, D., And Vaquero, M. A Hamilton-Jacobi theory for singular Lagrangian systems in the Skinner and Rusk setting. Int. J. Geom. Methods Mod. Phys. 9, 8 (2012), 1250074, 24.

[21] de León, M., Martín de Diego, D., And Vaquero, M. A Hamilton-Jacobi theory on Poisson manifolds. J. Geom. Mech. 6, 1 (2014), 121-140.

[22] de León, M., Martín de Diego, D., And Vaquero, M. Hamiton-Jacobi theory, Symmetries and Coisotropic reduction. Preprint. Part of this thesis, 2015.

[23] Eliashberg, Y., And Gromov, M. Lagrangian intersection theory: finitedimensional approach. In Geometry of differential equations, vol. 186 of Amer. Math. Soc. Transl. Ser. 2. Amer. Math. Soc., Providence, RI, 1998, pp. 27-118. 
[24] Evans, L. C. Weak KAM theory and partial differential equations. In Calculus of variations and nonlinear partial differential equations, vol. 1927 of Lecture Notes in Math. Springer, Berlin, 2008, pp. 123-154.

[25] Evans, L. C. Further PDE methods for weak KAM theory. Calc. Var. Partial Differential Equations 35, 4 (2009), 435-462.

[26] Evans, L. C. Partial differential equations, second ed., vol. 19 of Graduate Studies in Mathematics. American Mathematical Society, Providence, RI, 2010.

[27] Feng, K., And QIn, M. Symplectic geometric algorithms for Hamiltonian systems. Zhejiang Science and Technology Publishing House, Hangzhou; Springer, Heidelberg, 2010. Translated and revised from the Chinese original, With a foreword by Feng Duan.

[28] Fernandes, R. L., Ortega, J.-P., and Ratiu, T. S. The momentum map in Poisson geometry. Amer. J. Math. 131, 5 (2009), 1261-1310.

[29] Ferraro, S., de León, M., Marrero, J. C., Martín de Diego, D., and Vaquero, M. On the geometry of the Hamilton-Jacobi equation. Preprint. Part of this thesis, 2015.

[30] GE, Z. Generating functions, Hamilton-Jacobi equations and symplectic groupoids on Poisson manifolds. Indiana Univ. Math. J. 39, 3 (1990), 859-876.

[31] Ge, Z. Equivariant symplectic difference schemes and generating functions. Phys. D 49, 3 (1991), 376-386.

[32] Ge, Z., Kruse, H. P., Marsden, J. E., and Scovel, C. The convergence of Hamiltonian structures in the shallow water approximation. Canad. Appl. Math. Quart. 3, 3 (1995), 277-302.

[33] Ge, Z., and Marsden, J. E. Lie-Poisson Hamilton-Jacobi theory and Lie-Poisson integrators. Phys. Lett. A 133, 3 (1988), 134-139.

[34] Ge, Z., AND Scovel, C. Hamiltonian truncation of the shallow water equation. Lett. Math. Phys. 31, 1 (1994), 1-13.

[35] Goldstein, H. Classical mechanics, second ed. Addison-Wesley Publishing Co., Reading, Mass., 1980. Addison-Wesley Series in Physics.

[36] Hairer, E., Lubich, C., And Wanner, G. Geometric numerical integration, vol. 31 of Springer Series in Computational Mathematics. Springer, Heidelberg, 2010. Structure-preserving algorithms for ordinary differential equations, Reprint of the second (2006) edition. 
[37] Holm, D. D. Geometric mechanics. Part I, second ed. Imperial College Press, London, 2011. Dynamics and symmetry.

[38] Holm, D. D. Geometric mechanics. Part II. Rotating, translating and rolling, second ed. Imperial College Press, London, 2011.

[39] Hörmander, L. Fourier integral operators. I. Acta Math. 127, 1-2 (1971), 79-183.

[40] Iglesias-Ponte, D., De León, M., and Martín de Diego, D. Towards a Hamilton-Jacobi theory for nonholonomic mechanical systems. J. Phys. A 41, 1 (2008), 015205, 14.

[41] Marsden, J. E., And Ratiu, T. S. Introduction to mechanics and symmetry, second ed., vol. 17 of Texts in Applied Mathematics. Springer-Verlag, New York, 1999. A basic exposition of classical mechanical systems.

[42] McLachlan, R. I. Explicit Lie-Poisson integration and the Euler equations. Phys. Rev. Lett. 71, 19 (1993), 3043-3046.

[43] McLachlan, R. I., and Scovel, C. Equivariant constrained symplectic integration. J. Nonlinear Sci. 5, 3 (1995), 233-256.

[44] McLachlan, R. I., And Scovel, C. A survey of open problems in symplectic integration. In Integration algorithms and classical mechanics (Toronto, ON, 1993), vol. 10 of Fields Inst. Commun. Amer. Math. Soc., Providence, RI, 1996, pp. 151-180.

[45] Meyer, K. R. Generic bifurcation of periodic points. Trans. Amer. Math. Soc. 149 (1970), 95-107.

[46] Meyer, K. R. Equivariant generating functions and periodic points. In New trends for Hamiltonian systems and celestial mechanics (Cocoyoc, 1994), vol. 8 of Adv. Ser. Nonlinear Dynam. World Sci. Publ., River Edge, NJ, 1996, pp. 289-299.

[47] Moser, J., And Veselov, A. P. Discrete versions of some classical integrable systems and factorization of matrix polynomials. Comm. Math. Phys. 139, 2 (1991), $217-243$.

[48] Ohsawa, T., and Bloch, A. M. Nonholonomic Hamilton-Jacobi equation and integrability. J. Geom. Mech. 1, 4 (2009), 461-481.

[49] Schrödinger, E. Collected papers on wave mechanics. Chelsea Publishing Co., New York, 1982. Translated from the second German edition by J. F. Shearer and W. M. Deans, Including it Four lectures on wave mechanics.

[50] Scovel, C. On symplectic lattice maps. Phys. Lett. A 159, 8-9 (1991), 396-400. 
[51] Scovel, C. Symplectic numerical integration of Hamiltonian systems. In The geometry of Hamiltonian systems (Berkeley, CA, 1989), vol. 22 of Math. Sci. Res. Inst. Publ. Springer, New York, 1991, pp. 463-496.

[52] Scovel, C., And Weinstein, A. Finite-dimensional Lie-Poisson approximations to Vlasov-Poisson equations. Comm. Pure Appl. Math. 47, 5 (1994), 683-709.

[53] Vaisman, I. Lectures on the geometry of Poisson manifolds, vol. 118 of Progress in Mathematics. Birkhäuser Verlag, Basel, 1994.

[54] Viterbo, C. Symplectic topology as the geometry of generating functions. Math. Ann. 292, 4 (1992), 685-710.

[55] Viterbo, C. Solutions of Hamilton-Jacobi equations and symplectic geometry. Addendum to: Séminaire sur les Équations aux Dérivées Partielles. 1994-1995 [École Polytech., Palaiseau, 1995; MR1362548 (96g:35001)]. In Séminaire sur les Équations aux Dérivées Partielles, 1995-1996, Sémin. Équ. Dériv. Partielles. École Polytech., Palaiseau, 1996, p. 8.

[56] Weinstein, A. Lagrangian mechanics and groupoids. In Mechanics day (Waterloo, ON, 1992), vol. 7 of Fields Inst. Commun. Amer. Math. Soc., Providence, RI, 1996, pp. 207-231. 


\section{Scientific articles presented in this dissertation}

The scientific articles presented in this dissertation in partial fulfillment of the requirements for the degree of Doctor of Philosophy.

- Hamiton-Jacobi theory, Symmetries and Coisotropic Reduction, [22], arxiv.

- On the Geometry of the Hamilton-Jacobi equation and Generating Functions, [29], preprint.

- A Hamilton-Jacobi theory on Poisson manifolds ([21]). J. Geom. Mech. 6 (2014), no. 1, 121-140. article

- On the Hamilton-Jacobi theory for Singular Lagrangian Systems ([19]). J. Math. Phys. 54 (2013), no. 3, 032902, 32 pp. article

- A Hamilton-Jacobi theory for Singular Lagrangian Systems in the Skinner and Rusk setting ([20]). Int. J. Geom. Methods Mod. Phys. 9 (2012), no. 8, 1250074, 24 pp. article

Below, we give a brief introduction to the papers presented in this memory. Afterwards, the corresponding preprints are included.

\subsection{Hamiton-Jacobi theory, Symmetries and Coisotropic Reduction}

This chapter constitutes the initial point of this thesis. The results are contained in the paper [22]. As it was said in the abstract of this thesis, reduction theory is one of the oldest and most useful techniques in geometric mechanics, so it is natural to wonder if that theory can be extended to the Hamilton-Jacobi setting. The fist attempts in that direction were made by Ge and Marsden, who tried to solve that question in [33]. In that paper the authors use the invariance of the generating function, which is a complete solution of the Hamilton-Jacobi equation to obtain a reduced solution. An important observation there is that such reduced solution gives a Poisson transformation in the dual of the Lie algebra of the Lie group under consideration. 
This work had a profound impact to develop numerical methods for Lie algebras. Those methods are the analogous of the symplectic methods developed before using HamiltonJacobi theory. The importance of those methods is manifest by the vast number of papers based on this construction, [7, 11, 22, 34, 41, 42, 43, 44, 52]. That is justified by the utility of Lie-Poisson systems to model physical systems. The most well-known examples are the rigid body and the heavy top; more elaborated examples include infinite-dimensional systems like Euler or Poisson-Vlassov equations. Although the latter fall out of the scope of the theory, discretization of those equations can be carried out sometimes (see [52]) using Lie algrebras, and then the procedure can be applied to the discretized system. Moreover, one of the future work issues that we present is to discretize infinite-dimensional hamiltonian systems using a more general structure than Lie algebras, the Lie algebroids.

We propose a new approach, general enough to include the previous ones and to give new insight to develop more applications. The basic idea is to recall that if $S$ is a generating function, then $d S$ is a lagrangian submanifold and that there is a coistropic procedure for those submanifolds. With that idea in mind, it is easy to imagine how can be provide coisotropic submanifolds: just using momentum maps.

\subsection{On the Geometry of Hamilton-Jacobi theory}

This is perhaps the most important contribution of this thesis, the results are contained in [29]. There, motivated by the classical Hamilton-Jacobi theory and the Hamilton-Jacobi theory developed in the paper [22] (previous chapter), we introduce a general HamiltonJacobi theory for integrable Lie algebroids. These results provide a Hamilton-Jacobi theory that covers the main examples:

- Lie algebras, to deal with Lie-Poisson systems, think about the rigid body as a classical example.

- Action Lie algebroids, which are nice to deal with some kind of symmetries, like in the heavy-top case.

- Atiyah algebroids, to deal with symmetries in cotangent bundles, like the Toda lattice and others.

Shortly after working ot this topic, we found out that Scovel and Weinstein had similar ideas in [52]. The main ideas come from symplectic groupoids and Lie algebroids, as it can be concluded from this text taken from that paper

"Our first derivation used the theory of Lie groupoids and Lie algebroids, and the Poisson structures on the duals of Lie algebroids. We were then able to eliminate the oid theory in favor of more well-known ideas on Poisson reduction. [...] The groupoid aspect of the theory also provides natural Poisson maps, useful in the application of Ruth type integration techniques, which do not seem easily derivable from the general theory of Poisson reduction". 


\section{Scovel and A. Weinstein [52], p. 683.}

However, they did not go further in the development of the Poisson integrators. Their work focuses on the truncation of the Poisson-Vlasov system. It is worth mentioning to quote them again

"Given a Poisson manifold $P$, there exists a (local) groupoid $G$ over $P$, with a symplectic structure on $G$ for which $\alpha$ and $\beta$ are Poisson and anti-Poisson maps respectively. A generating function for a Poisson map is a lagrangian submanifold of $G$ which projects diffeomorphically under both $\alpha$ and $\beta$ near the point of interest in $P$. The Poisson map is then $\beta \circ \alpha^{-1}$ restricted to the lagrangian submanifold. The determination of the lagrangian submanifold from the Hamiltonian follows from the appropriate Hamilton-Jacobi theory; see [30]."

\section{Scovel and A.D. Weinstein [52], p. 707.}

So it seems that A.D. Weinstein had already the idea in mind about how to extend the classical theory to the Poisson setting. Although in [30] some first steps are given, the work there is far from complete. In [29] we develop the whole theory. We were also able to introduce generating functions for Poisson mappings. Our generating functions solve affirmatively an open problem of the area:

"Is there a generating function for Lie-Poisson maps which generates the identity map via the coadjoint action of the identity group element?"

R.I. McLachlan and C. Scovel. [44], pag 157.

Those generating functions are analogues of the classical non free generating functions. The theory is based on previous works by Channell, Ge, Marsden, Scovel, and Weinstein among others $([7,11,33,42,52])$. Our framework has a very high applicability, we can show not only that we can obtain some beautiful theoretical results from the geometric viewpoint, but that we can provide accurate Poisson integrators. The fact is that even when the Hamilton-Jacobi equation cannot be analytically solved, it can always be approximated and you can obtain a Poisson integrator out of it. All these results and the previously mentioned applications will appear in [29]. This also makes tools, before restricted to symplectic geometry, now available for Poisson systems.

\subsection{A Hamilton-Jacobi theory on almost-Poisson Man- ifolds}

The final objective of Chapter 2 and Chapter 3 was to develop a reduction theory and extend the classical Hamilton-Jacobi theory to the Poisson setting. In this chapter we extend the results sketched in the Chapter 1, (Introduction, Section 1.4.1 and Section 1.4.2) to the almost-Poisson framework. The results are contained in the paper [17]. The 
main application of that extensions is to non-holonomic mechanics. Moreover, as a byproduct of this work we can recover the results in [18, 40, 48, 9], from a very neat geometric viewpoint. The bottom line is that the classical symplectic interpretation of the HamiltonJacobi equation holds, mutatis mutandis, to the almost-Poisson systems. The lagrangian manifolds in the almost-Poisson case are defined in a natural way (taken from [53]), but a proposition analogous to 4 can be formulated in this setting. This new result, not only provides the aforementioned extension, it can be exploited to add forces to the system, among other structures.

\subsection{Hamilton-Jacobi for Singular Lagrangians}

In this section we introduce the last extension of the Hamilton-Jacobi theory of this dissertation. The goal is to get a Hamilton-Jacobi theory for singular lagrangian systems. Singular lagrangian systems and the Gotay-Nester-Hinds algorithm of constraints are very important in classical field theory. Due to that importance, it seems natural to try such extension first in the classical mechanics setting.

In the papers $[19,20]$ we develop such theory. We introduce the Gotay-Nester-Hinds algorithm in full detail and solve some simple examples. Comparisons with Hinds algorithm are given. Moreover, the lagrangian and hamiltonian counterparts are analyzed in [20] and even the Skinner and Rusk setting is included in [19]. With these results we deepen in some previous works, like [9].

In the last part of this memory we shall include a list of conclusions and some future work.

\subsection{Other contributions}

Besides working on the Hamilton-Jacobi theory, during my PhD I had the chance to work on other topics non related directly to my thesis. Here I list some of them

1. Classical field theory: During my stay at PIMS (Vancouver) I learnt some classical field theory from the geometric viewpoint. In particular, I related the multimomentum mapping with the stress-energy-momentum tensor for second order classical field theories, obtaining also a neat description of the Komar mass in general relativity. Those results will appear soon in arxiv, in a joint paper with Mark J. Gotay.

2. Stablity of leaves: During my stay at UIUC I could learn from R. Loja-Fernandes, one of the leader mathematicians in the field of Lie algebroids and Poisson geometry, some topics related to the permanence of certain compact leaves of the characteristic distribution of a Lie algebroid. That led to an ongoing project, that will be developed during the next years. 
3. Lagrangian intersection: As one of the organizers of the group of Geometric Mechanics Seminar at ICMAT I gave several talks about some work by A. Weinstein and relations between lagrangian intersection and dynamical systems. That led to some problems in dynamical systems, that will be treated during the next years. 


\title{
Hamilton-Jacobi theory, Symmetries and Coisotropic Reduction
}

\author{
Manuel de León, David Martín de Diego \\ Instituto de Ciencias Matemáticas, ICMAT, \\ c) Nicolás Cabrera, no 13-15, Campus Cantoblanco,UAM, \\ 28049 Madrid, Spain
}

\begin{abstract}
Reduction theory has played a major role in the study of Hamiltonian systems. On the other hand, the Hamilton-Jacobi theory is one of the main tools to integrate the dynamics of certain hamiltonian problems and a topic of research on its own. Moreover, the construction of several symplectic integrators relies on approximations of a complete solution of the Hamilton-Jacobi equation. The natural question that we address in this paper is how these two topics (reduction and Hamilton-Jacobi theory) fit together. We obtain a reduction and reconstruction procedure for the Hamilton-Jacobi equation with symmetries, even in a generalized sense to be clarified below. Several applications and relations to other reduction of the Hamilton-Jacobi theory are shown in the last section of the paper. It is remarkable that as by-product we obtain a generalization of the Ge-Marsden reduction procedure ([18]) and the results in [17]. Quite surprinsingly, the classical ansätze available in the literature to solve the Hamilton-Jacobi equation (see $[2,19]$ ) are also particular instances of our framework.
\end{abstract}

Keywords: Hamilton-Jacobi theory, reduction, momentum mapping, symmetries, lagrangian submanifolds.

\footnotetext{
*mdeleon@icmat.es

†david.martin@icmat.es

‡miguel.vaquero@icmat.es
} 


\section{Contents}

1 Introduction $\quad 2$

2 Preliminaries 4

2.1 Lifted actions to $T Q$ and $T^{*} Q \ldots \ldots \ldots \ldots$

2.2 Momentum Mapping. . . . . . . . . . . . . . . 5

3 The Hamilton-Jacobi Equation 9

3.1 Generalized Solutions . . . . . . . . . . . . . . . . . . . . 9

3.2 Invariant $G$-solutions . . . . . . . . . . . . . . . . . 10

3.3 Reduction and Reconstruction . . . . . . . . . . . . . . . 11

4 Reduction of H-J equation and reduction of dynamics 15

5 Examples $\quad 17$

5.1 Lie groups . . . . . . . . . . . . . . . . . . . 17

5.2 The trivial case: $Q=M \times G \ldots \ldots \ldots 17$

5.2.1 Time-dependent H-J solution for time-independent systems 18

5.3 Complete Solutions _. . . . . . . . . . . . . . . 18

5.3.1 Other types of generating function . . . . . . . . . . . . 22

5.4 Calogero-Moser system . . . . . . . . . . . . . 24

6 Conclusions and Future Research 26

$\begin{array}{ll}\text { Appendix A Principal bundles and adjoint bundles } & 26\end{array}$

$\begin{array}{ll}\text { Appendix B Magnetic Terms } & 27\end{array}$

\section{Introduction}

The Hamilton-Jacobi theory is today a well-known theory by mathematicians and physicist. The equations

$$
\begin{aligned}
& \text { 1. } H\left(q^{i}, \frac{\partial S}{\partial q^{i}}\left(q^{i}\right)\right)=E, \\
& \text { 2. } \frac{\partial S}{\partial t}+H\left(t, q^{i}, \frac{\partial S}{\partial q^{i}}\left(t, q^{i}\right)\right)=E
\end{aligned}
$$

appear in any classical mechanics book, like [1, 19]. The Hamilton-Jacobi theory is connected to geometric optics and to classical and quantum mechanics in several intriguing ways. In geometric optics it establishes the link between particles and waves through the characteristic function, [21]. Hamilton and Jacobi extended this duality (wave-particle) to classical mechanics, where a solution of the Hamilton-Jacobi equation allows the reduction of the number of equations of motion by half, and a complete solution of the Hamilton-Jacobi equation allows us to make a change of variables that makes the integration of 
Hamilton's equations trivial (usually called "a transformation to equilibrium"). A detailed account of these topics can be found in [1,3]. Recently the HamiltonJacobi theory has also been extended to the non-holonomic setting, $[6,11,10$, 22].

The Hamilton-Jacobi theory and the theory of generating functions also gave rise to families of symplectic numerical integrators which over long times are clearly superior to other methods (see $[7,12,18]$ ). Extending those integrators to the Lie-Poisson setting motivated the beginning of the reduction of the Hamilton-Jacobi theory in [17, 18], by Z. Ge and J.E. Marsden. After their approach, several works appeared along the same lines, $[4,8,26,27,30]$. Although we are not dealing with numerical methods, getting a deeper understanding of those results motivated this work to some extent. Moreover, a general setting to develop numerical methods based on the Hamilton-Jacobi theory for (integrable) Poisson manifolds will appear elsewhere [14]. The importance of the development of such geometric-Poisson integrators is beyond any doubt, taking into account the success of their symplectic analogues.

On the other hand, reduction theory is still nowadays an important topic of research. Since Jacobi's elimination of the node, and its formalization through the Meyer-Marsden-Weinstein reduction, the usefulness of the theory is widely known. A complete reference for Hamiltonian reduction is [25].

The present paper studies how to apply reduction theory to simplify the Hamilton-Jacobi equation via the coisotropic reduction of lagrangian submanifolds (see [33]). We combine the aforementioned coisotropic reduction and cotangent bundle reduction to obtain the reduced Hamilton-Jacobi equation, which turns out to be an algebraic-PDE equation. As mentioned above, previous attempts to obtain a reduction of the (complete solutions of the) HamiltonJacobi equation were carried out by Ge and Marsden in [18] in order to provide a setting to develop Lie-Poisson integrators. Nonetheless, they only work out the details in the case where the configuration manifold is a Lie group, although they claim that the general procedure can be obtained. The main difference between their and our approach is that while Ge and Marsden reduce the generating function, say $S$, we focus on the corresponding lagrangian submanifold, say $\operatorname{Im}(d S)$, that allows us to obtain a more general setting of wide applicability. For instance, generating functions which are not of type $I$, in the language of [19] can be treated using our approach, while this seems not to be the case for the previous settings. Of course, Ge's framework can be obtained from our results in a straightforward fashion as will be shown in the last section, where we also deal with some examples, like a two particles Calogero-Moser system. Finally, although we did not include it here, the results by H. Wang in [32] are a particular case of our framework as well. The use of generating families to obtain lagrangian submanifolds ([9]) is another interesting topic not treated here that fits into our work.

The paper is organized as follows. In Section 2 we provide the necessary preliminaries and we establish the notation and conventions that we follow during the rest of the paper. In Section 3 we introduce the Hamilton-Jacobi equation and the announced reduction and reconstruction procedure. In Section 4 we 
show that the "two reduced dynamics" are related in the expected way. Section 5 is devoted to applications and examples. We also include two appendices about adjoint bundles and magnetc terms to make the paper self-contained.

\section{Preliminaries}

In this paper all manifolds and mappings are supposed to be infinitely differentiable $\left(C^{\infty}\right)$. Given a map $f: M \rightarrow N$ between manifolds $M$ and $N$, we will use the notation $T f$ to denote the tangent map $(T f: T M \rightarrow T N)$, and $T f(p)$, where $p$ is a point on $M$, to denote the tangent map at that point $T f(p): T_{p} M \rightarrow T_{f(p)} N$. Given a vector field on the manifold $M$, say $X$, the evaluation of that vector field at a point $p \in M$ will read $X(p)$. The flow of the vector fields under consideration will be assumed to be defined globally, although our results hold for locally defined flows with the obvious modifications. Along this paper $G$ will be a connected Lie group and $\mathfrak{g}$ the corresponding Lie algebra. We will make use of $A d^{*}$ to represent the Coadjoint action on the dual of $\mathfrak{g}$ given by

$$
\begin{aligned}
A d^{*}: G \times \mathfrak{g}^{*} & \longrightarrow \mathfrak{g}^{*} \\
(g, \mu) & \rightarrow A d_{g}^{*}(\mu)=\mu \circ T R_{g} \circ T L_{g^{-1}},
\end{aligned}
$$

where $L_{g}(h)=g \cdot h$ and $R_{g}(h)=h \cdot g$ are the left and right multiplication on the group $G$. Notice that the Coadjoint action is a left action. Given $\mu \in \mathfrak{g}^{*}$, $\operatorname{Orb}^{A d^{*}}(\mu)$ denotes the orbit by the Coadjoint action through $\mu$.

\subsection{Lifted actions to $T Q$ and $T^{*} Q$}

Let $G$ be a connected Lie group acting freely and properly on a manifold $Q$ by a left action $\Phi$

$$
\begin{aligned}
\Phi: G \times Q & \rightarrow Q \\
(g, q) & \rightarrow \Phi(g, p)=g \cdot p
\end{aligned}
$$

Given $g \in G$, we denote by $\Phi_{g}: Q \rightarrow Q$ the diffeomorphism defined by $\Phi_{g}(q)=$ $\Phi(g, q)=g \cdot q$. Recall that under these conditions the quotient $Q / G$ can be endowed with a manifold structure such that the canonical projection $\pi: Q \rightarrow$ $Q / G$ is a $G$-principal bundle. The action $\Phi$ introduced above can be lifted to actions on the tangent and cotangent bundles, $\Phi^{T}$ and $\Phi^{T^{*}}$ respectively. We briefly recall here their definitions.

- Lifted action on $T Q$. We introduce the action $\Phi^{T}: G \times T Q \rightarrow T Q$ such that $\Phi_{g}^{T}: T Q \rightarrow T Q$ is defined by

$$
\Phi_{g}^{T}\left(v_{q}\right)=T \Phi_{g}(q)\left(v_{q}\right) \in T_{g q} Q \quad \text { for } v_{q} \in T_{q} Q .
$$

-Lifted action on $T^{*} Q$. Analogously, we introduce the following action 
$\Phi^{T^{*}}: G \times T^{*} Q \rightarrow T^{*} Q$ such that $\Phi_{g}^{T^{*}}: T^{*} Q \rightarrow T^{*} Q$ is defined by

$$
\Phi_{g}^{T^{*}}\left(\alpha_{q}\right)=\left(T \Phi_{g^{-1}}\right)^{*}(g q)\left(\alpha_{q}\right) \in T_{g q}^{*} Q \quad \text { for } \alpha_{q} \in T_{q}^{*} Q .
$$

Both actions can be easily checked to be free and proper. If $\alpha_{q} \in T^{*} Q$, we will denote the orbit through $\alpha_{q}$ by $\operatorname{Orb}\left(\alpha_{q}\right)$.

\subsection{Momentum Mapping}

As is well-known, there exists a $G$-equivariant momentum mapping for the above action on $T^{*} Q$ with respect to its canonical symplectic form, from now on denoted by $\omega_{Q}$. This momentum map is given by $J: T^{*} Q \rightarrow \mathfrak{g}^{*}$ where $J\left(\alpha_{q}\right)$ is such that $J\left(\alpha_{q}\right)(\xi)=\alpha_{q}\left(\xi_{Q}(q)\right)$ for $\xi \in \mathfrak{g}$. Here $\xi_{Q}$ is the vector field on $Q$ determined via the action $\Phi$, called the infinitesimal generator. The integral curve of $\xi_{Q}$ passing through $q \in Q$ is just $t \rightarrow \exp (t \xi)(q)$.

Given $\xi \in \mathfrak{g}$, we denote by $J_{\xi}: T^{*} Q \rightarrow \mathbb{R}$ the real function obtained by the pairing between $\mathfrak{g}$ and $\mathfrak{g}^{*}, J_{\xi}\left(\alpha_{q}\right)=\left\langle J\left(\alpha_{q}\right), \xi\right\rangle$. By the definition of momentum mapping we have $\xi_{T^{*} Q}=X_{J_{\xi}}$, where $\xi_{T^{*} Q}$ is the fundamental vector field generated by $\xi$ via the action $\Phi^{T^{*}}$. Indeed, we have $i_{\xi_{T^{*}}} \omega_{Q}=d J_{\xi}$ and $X_{J_{\xi}}$ is the vector field satisfying $i_{X_{J_{\xi}}} \omega_{Q}=d J_{\xi}$.

The next proposition, combined with the fact that $\Phi^{T^{*}}$ is free and $G$ connected, ensures that for a connected Lie group every $\mu \in \mathfrak{g}^{*}$ is a regular value and so $J^{-1}(\mu)$ is a submanifold. In fact, the next proposition characterizes regular values of momentum mappings taking into account the infinitesimal behavior of the symmetries. We define $\mathfrak{g}_{p}=\left\{\xi \in \mathfrak{g}\right.$ such that $\left.\xi_{Q}(p)=0\right\}$.

Proposition 1 (Marsden et al. [25]) Let $(M, \Omega)$ be a symplectic manifold and $G$ a Lie group which acts by symplectomorphism with equivariant momentum map $J$. An element $\mu \in \mathfrak{g}^{*}$ is a regular value of $J$ iff $\mathfrak{g}_{p}=\{0\}$ for all $p \in J^{-1}(\mu)$.

Proof: Let $p \in J^{-1}(\mu)$ and assume that $\mathfrak{g}_{p}=0$, then we will show that $T J(p)$ is surjective. This is equivalent to proving that the anihilator of $\operatorname{Im}(T J(p))$ is $\{0\}$. Assume that $\xi \in \mathfrak{g}$ is such that the natural pairing $\langle T J(p)(X), \xi\rangle=0$ for all for all $X \in T_{p} S$. That means that $T J(p)(X)(\xi)=0$ or that $\Omega\left(X(p), \xi_{M}(p)\right)=0$ for all $X \in T_{p} S$. Since $\Omega$ is non-degenerate that means $\xi_{M}(p)=0$ and so $\xi \in \mathfrak{g}_{p}$ and therefore $\xi=0$ by hypothesis. Reversing the computation the converse easily follows.

Remark 1 In the case that concerns us, namely $\left(T^{*} Q, \omega_{Q}\right)$ with the action $\Phi^{T^{*}}$, the previous theorem says that $J^{-1}(\mu)$ is always a submanifold of $T^{*} Q$.

Now we introduce $G$-invariant lagrangian submanifolds and the main results about them. The main results of this paper will be direct applications of these results. 
Definition 2 Assume as above that the triple $\left(T^{*} Q, \omega_{Q}, h\right)$ is endowed with a hamiltonian action $\Phi$. A G-invariant lagrangian submanifold is a lagrangian submanifold $L$ in $T^{*} Q$ such that for all $g \in G$ we have $\Phi_{g}^{T^{*}}(L)=L$.

We give a characterization of $G$-invariant lagrangian submanifolds in terms of equivariant momentum mappings. The next result should be considered as a generalization of the Hamilton-Jacobi theory, an explanation for this claim will be given in Remark 2. More detailed results in this direction are given in [15].

Lemma 3 Under the previous assumptions, let $L \subset T^{*} Q$ be a lagrangian submanifold of $\left(T^{*} Q, \omega_{Q}\right)$. Then $J$ is constant along $L$ if and only if $L$ is $G$ invariant.

Proof: Let be $\alpha_{q} \in L$ and $X \in T_{\alpha_{q}} L$, then

$$
d J_{\xi}\left(\alpha_{q}\right)(X)=\left(i_{\xi_{T^{*} Q}} \omega_{Q}\right)\left(\alpha_{q}\right)(X)=\omega_{Q}\left(\alpha_{q}\right)\left(\xi_{T^{*} Q}\left(\alpha_{q}\right), X\right) .
$$

Now, notice that

$$
\xi_{T^{*} Q}\left(\alpha_{q}\right)=\text { tangent vector at } t=0 \text { to the curve } \exp (t \xi)\left(\alpha_{q}\right) .
$$

Since $\exp (t \xi)\left(\alpha_{q}\right)$ is contained in the orbit of $\alpha_{q} \in L$, and $\operatorname{Orb}\left(\alpha_{q}\right) \subset L$ since $L$ is $G$-invariant (that is, $G \cdot L \subset L$ ), we deduce that $\xi_{T^{*} Q}\left(\alpha_{q}\right) \in T_{\alpha_{q}} L$. Therefore, (1) vanishes since $L$ is lagrangian. Finally, since $J_{\xi}$ is constant along $L$, we have $J_{\xi}\left(\alpha_{q}\right)=c_{\xi}$ for all $\alpha_{q} \in L$ and for all $\xi \in \mathfrak{g}$ and thus, $J\left(\alpha_{q}\right)=\mu$ for all $\alpha_{q} \in L$ (such that $\left.\mu(\xi)=c_{\xi}\right)$. Reversing the computations we obtain the other implication.

Remark 2 Notice that the Hamilton-Jacobi theory itself is a particular case of the theorem above, which should be considered as a generalization of that theory. Let us clarify this assertion, we have a hamiltonian system $\left(T^{*} Q, \omega_{Q}, h\right)$, with the associated hamiltonian vector field $X_{h} \in \mathfrak{X}\left(T^{*} Q\right)$; we denote the flow of $X_{h}$ by $\Psi^{h}: \mathbb{R} \times T^{*} Q \rightarrow T^{*} Q$, recall that the flow is just an $\mathbb{R}$ action on $T^{*} Q$. By Liouville's Theorem this action is hamiltonian and it is easy to see that the hamiltonian $h$ is a momentum map for that action. If we seek a $\mathbb{R}$ invariant lagrangian submanifold, say $L$, then, by Lemma $3 h_{\mid L}=E$, where $E$ is a constant. Moreover, assume that $L=\operatorname{Im}(d S)$ where $S: Q \rightarrow \mathbb{R}$ is a real function, then we recover the classical Hamilton-Jacobi equation

$$
H\left(q^{i}, \frac{\partial S}{\partial q^{i}}\left(q^{i}\right)\right)=E .
$$

The time-dependent and complete solutions cases of the Hamilton-Jacobi equation follow by an analogous construction.

Remark 3 Most of the results in [17] can be recovered from Lemma 3. Indeed, there the author claims that there is a deep connection between the symmetry 
of a symplectic difference scheme and the preservation of first integrals. For instance, in [17] p. 378, the following theorem is stated

Theorem A symplectic difference scheme preserves a function $f$ up to a constant

$$
f \circ D_{h}=f+c
$$

iff the scheme is invariant under the phase flow of $f$.

Assume a hamiltonian system $(M, \Omega, H)$. Then a symplectic scheme (following [17], p. 377), after fixing Darboux coordinates on $M$, is a rule which assigns to every hamiltonian function a symplectic map depending smoothly on a paramenter $\tau$, called the time step. A symplectic difference scheme is denoted in [17] by $D_{h}^{\tau}$. This means that a symplectic difference scheme is just a lagrangian submanifold $\operatorname{graph}\left(D_{h}^{\tau}\right)=L \subset M \times M$, with the symplectic structure $\bar{\Omega}=\pi_{1}^{*} \Omega-\pi_{2}^{*} \Omega$ on $M \times M$, where $\pi_{i}: M \times M \rightarrow M$ are the corresponding projections over the $i$-factor. Consider now $\bar{f}=\pi_{1}^{*} f-\pi_{2}^{*} f$, it is obvious that $f$ is preserved by $D_{h}^{\tau}$ iff $\bar{f}$ is constant along graph $\left(D_{h}^{\tau}\right)=L$. By definition $X_{\bar{f}}=\left(X_{f}, X_{f}\right)$ and a straightforward application of Lemma 3, taking into account that $\bar{f}$ is the moment of the action give by the flow of $X_{\bar{f}}$, gives that $\bar{f}$ is constant along $L$ iff $L$ is invariant under the flow of $X_{\bar{f}}$. Recalling that the first statement is equivalent to $f$ being preserved by the symplectic scheme and the second claim is equivalent to saying that the scheme is invariant under the phase flow we recover the main "principle" of [17].

Remark 4 Assume that we are in the hypothesis of the above lemma. If $J(L)=$ $\{\mu\}, \mu \in \mathfrak{g}^{*}$, then we deduce that $\mu$ is a fixed point for the Coadjoint action $A d^{*}: G \rightarrow \operatorname{Aut}\left(\mathfrak{g}^{*}\right)$. Indeed, remember that $J$ is $G$-equivariant, that is, the following diagram is commutative

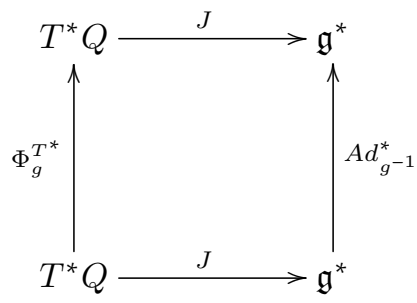

Then $A d_{g^{-1}}^{*}(\mu)=A d_{g^{-1}}^{*} J\left(\alpha_{q}\right)=J\left(\Phi_{g}^{T^{*}}\left(\alpha_{q}\right)\right)=\mu$, for all $g \in G$.

Lemma 4 If $\mu$ is such that $G_{\mu}=G$, then $J^{-1}(\mu)$ is a coisotropic submanifold $\left(G_{\mu}\right.$ denotes the isotropy group with respect to the Coadjoint action).

Proof: Given any point $\alpha_{q} \in J^{-1}(\mu)$, we have 


$$
\begin{aligned}
T_{\alpha_{q}} J^{-1}(\mu) & =\operatorname{ker}\left(T J\left(\alpha_{q}\right)\right)=\left\{X \in T_{\alpha_{q}}\left(T^{*} Q\right) \text { such that } T J\left(\alpha_{q}\right)(X)=0\right\} \\
& =\left\{X \in T_{\alpha_{q}}\left(T^{*} Q\right) \text { such that } T J\left(\alpha_{q}\right)(X)(\xi)=0 \text { for all } \xi \in \mathfrak{g}\right\} \\
& =\left\{X \in T_{\alpha_{q}}\left(T^{*} Q\right) \text { such that } \omega\left(\alpha_{q}\right)\left(X, \xi_{T^{*} Q}\right)=0 \text { for all } \xi \in \mathfrak{g}\right\} \\
& =\left(\operatorname{TOrb}\left(\alpha_{q}\right)\right)^{\perp}
\end{aligned}
$$

since $\xi_{T^{*} Q}\left(\alpha_{q}\right)$ generates the orbit through $\alpha_{q}$. Therefore, we have $\left(T_{\alpha_{q}} J^{-1}(\mu)\right)^{\perp}$ $=T_{\alpha_{q}} \operatorname{Orb}\left(\alpha_{q}\right)$ for all $\alpha_{q} \in J^{-1}(\mu)$. But $J$ is $G$-equivariant and $G=G_{\mu}$, thus $J\left(\Phi_{g}^{T^{*}}\left(\alpha_{q}\right)\right)=A d_{g^{-1}}^{*} J\left(\alpha_{q}\right)=A d_{g^{-1}}^{*} \mu=\mu$ and so $\Phi_{g}^{T^{*}}\left(\alpha_{q}\right) \in J^{-1}(\mu)$. Then, $\operatorname{Orb}\left(\alpha_{q}\right) \subset J^{-1}(\mu)$, and thus $T_{\alpha_{q}} \operatorname{Orb}\left(\alpha_{q}\right) \subset T_{\alpha_{q}} J^{-1}(\mu)$. Consequently, we have $\left(T_{\alpha_{q}} J^{-1}(\mu)\right)^{\perp}=T_{\alpha_{q}} \operatorname{Orb}\left(\alpha_{q}\right) \subset T_{\alpha_{q}}\left(J^{-1}(\mu)\right)$ and we conclude that $J^{-1}(\mu)$ is coisotropic.

The next result is a well-known theorem in symplectic geometry, see [23, 33]. It will allow us to carry out our reduction procedure in a straightforward way.

Theorem 5 (Coisotropic Reduction) Let $(M, \omega)$ be a symplectic manifold, $C \subset M$ a coisotropic submanifold and $C / \sim$ the quotient space of $C$ by the characteristic distribution $D=k e r\left(\omega_{\mid C}\right)$; we shall denote by $\pi: C \rightarrow C / \sim$ the canonical projection and by $\omega_{C}$ the natural projection of $\omega$ to $C / \sim$ (notice that $\left(C / \sim, \omega_{C}\right)$ is again a symplectic manifold, assuming that it is again a manifold). Assume that $L \subset M$ is a lagrangian submanifold such that $L \cap C$ has clean intersection, then $\pi(L \cap C)$ is a lagrangian submanifold of $\left(C / \sim, \omega_{C}\right)$.

The following diagram illustrates the above situation

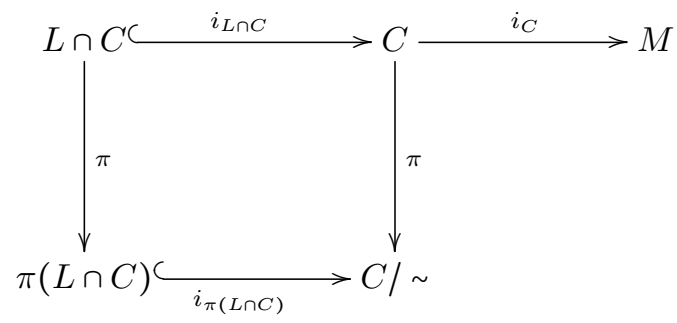

We can apply this theorem to the situation described before. Indeed, given $\mu \in \mathfrak{g}^{*}$ such that it is a fixed point of the Coadjoint action (i.e. $A d_{g}^{*}(\mu)=\{\mu\}$ for all $g \in G$ ), then we have the following diagram, since by Lemma 4 we know 
that $J^{-1}(\mu)$ is coisotropic:

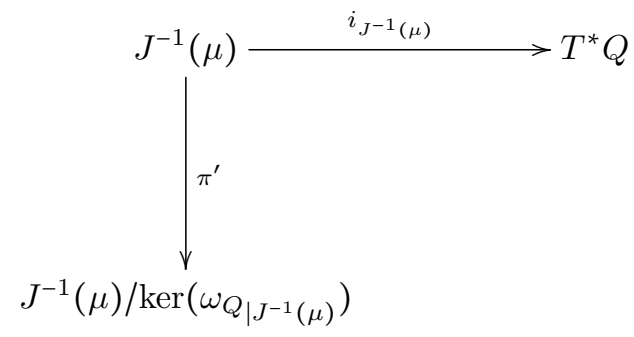

But $\operatorname{ker}\left(\omega_{Q_{\mid J^{-1}(\mu)}}\right)\left(\alpha_{q}\right)=\left(T_{\alpha_{q}} J^{-1}(\mu)\right)^{\perp}=T_{\alpha_{q}} \operatorname{Orb}\left(\alpha_{q}\right)$ for all $\alpha_{q} \in J^{-1}(\mu)$, and since $G=G_{\mu}$, we can see that $J^{-1}(\mu) / \operatorname{ker}\left(\omega_{Q \mid J^{-1}(\mu)}\right)=J^{-1}(\mu) / G$. But this is just the symplectic reduction of $T^{*} Q$ according to the Marsden-Weinstein reduction theorem, see [24].

\section{The Hamilton-Jacobi Equation}

\subsection{Generalized Solutions}

Along this section $h: T^{*} Q \rightarrow \mathbb{R}$ will be a hamiltonian function. We are going to use the previous results to carry out our reduction of the Hamilton-Jacobi equation. By Hamilton-Jacobi equation we mean

1. The time-independent Hamilton-Jacobi equation:

$$
h\left(q^{i}, \frac{\partial S}{\partial q^{i}}\left(q^{i}\right)\right)=E .
$$

2. The time-dependent Hamilton-Jacobi equation:

$$
\frac{\partial S}{\partial t}+h\left(t, q^{i}, \frac{\partial S}{\partial q^{i}}\left(t, q^{i}\right)\right)=E .
$$

3. A complete solution of the Hamilton-Jacobi equation: that is, a real-valued function $S\left(t, q^{i}, \alpha^{i}\right)$ depending on as many parameters $\left(\alpha^{i}\right)$ as the dimension of the configuration manifold, such that

(a) For every (fixed) value of the parameters $\left(\alpha^{i}\right), S\left(t, q^{i}, \alpha^{i}\right)$ satisfies the time-dependent Hamilton-Jacobi equation,

$$
\frac{\partial S}{\partial t}\left(t, q^{i}, \alpha^{i}\right)+h\left(t, q^{i}, \frac{\partial S}{\partial q^{i}}\left(t, q^{i}, \alpha^{i}\right)\right)=0 .
$$

(b) The non-degeneracy condition: consider the matrix whose components $(i, j)$ are given by $\frac{\partial^{2} S}{\partial q^{i} \partial \alpha^{j}}$, that we denote by $\left(\frac{\partial^{2} S}{\partial q^{i} \partial \alpha^{j}}\right)$, then

$$
\operatorname{det}\left(\frac{\partial^{2} S}{\partial q^{i} \partial \alpha^{j}}\right) \neq 0
$$


We define below the concept of generalized solution, which is a generalization of a solution of the time-independent Hamilton-Jacobi equation (see [5]), and we develop our theory for this case. Analogous procedures hold for the timeindependent Hamilton-Jacobi equation and for the complete solutions cases, as both settings can be (almost) considered as particular cases of the timeindependent Hamilton-Jacobi theory. Along the examples section, sections 5.2.1 and 5.3, we will make this claim explicit.

Definition 6 We say that a submanifold $L \subset T^{*} Q$ is a solution of the (timeindependent) Hamilton-Jacobi problem for $h$, if:

- $L$ is a lagrangian submanifold of $T^{*} Q$.

- $h$ is constant along $L$.

A solution $L$ of the Hamilton-Jacobi equation for $h$ is horizontal if $L=\operatorname{Im}(\gamma)$, being $\gamma$ a 1-form on $Q$.

Remark 5 Let us describe with more detail the case of horizontal solutions, that is, when $L=\operatorname{Im}(\gamma), \gamma$ a 1 -form on $Q$. Recall that $\operatorname{Im}(\gamma)$ is lagrangian if and only if $\gamma$ is closed, so locally

$$
\gamma=d S .
$$

Therefore, the condition $h_{\mid \operatorname{Im}(\gamma)}=$ cte, can be equivalently written as

$$
h \circ \gamma=c t e
$$

or

$$
h\left(q^{i}, \frac{\partial S}{\partial q^{i}}\right)=c t e
$$

which is the usual form of the Hamilton-Jacobi equation. This fact justifies the definition above.

Remark 6 Notice that the fact that a horizontal lagrangian submanifold $L$ is $G$-invariant does not imply that its generating function is invariant too. In fact, its generating function will be invariant iff $J(L)=0$. Since $J \circ d S=$ $\mu$, then $d S(q)\left(\xi_{Q}(q)\right)=J \circ d S(q)\left(\xi_{T^{*} Q}(q)\right)=\mu(\xi)$, which only vanish for all $\xi \in \mathfrak{g}$ if $\mu=0$. Here the advantages of dealing with lagrangian submanifolds instead of functions are already manifest, as there are $G$-invariant lagrangian manifolds whose generating function is not $G$-invariant, see Section 5 . Notice that invariance of the generating function has been assumed in [17, 18].

\subsection{Invariant $G$-solutions}

We assume now that a Lie group $G$ acts on $Q$ such that the action is free and proper. Given $\mu \in \mathfrak{g}$, then $J^{-1}(\mu)$ is a submanifold of $T^{*} Q$. We can summarize 
the situation in the following diagram:

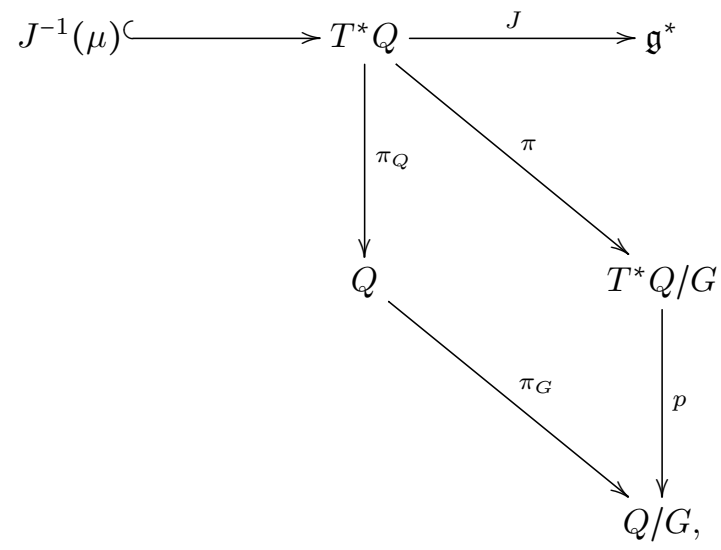

where $\pi_{Q}, \pi, \pi_{G}$ and $p$ are the canonical projections. We will use $\pi^{\prime}$ for the projection

$$
\pi^{\prime}=\pi_{\mid J^{-1}(\mu)}: J^{-1}(\mu) \rightarrow T^{*} Q / G .
$$

As we know, $T^{*} Q / G$ has a Poisson structure induced by the canonical symplectic structure on $T^{*} Q$, such that.

$$
\pi: T^{*} Q \rightarrow T^{*} Q / G
$$

is a Poisson morphism (see [25] for the details). The next proposition shows the symplectic structure of the leaves of the characteristic distribution of the Poisson structure of $T^{*} Q / G$.

Proposition 7 (Marsden et al. [23, 25, 31]) The symplectic leaves of $T^{*} Q / G$ are just the quotient spaces $\left(J^{-1}\left(\operatorname{Orb}^{A d^{*}}(\mu)\right)\right) / G$.

\subsection{Reduction and Reconstruction}

Assume now that $\mu$ is a fixed point for the Coadjoint action, i.e. $\operatorname{Orb}^{A d^{*}}(\mu)=$ $\{\mu\}$. Then $J^{-1}(\mu) / G$ is a symplectic leaf of $T^{*} Q / G$. Assume now that $L \subset$ $J^{-1}(\mu)$ is a lagrangian submanifold; since $J^{-1}(\mu)$ is a coisotropic submanifold of $\left(T^{*} Q, \omega_{Q}\right)$, we deduce that $\pi(L)$ is a lagrangian submanifold of the quotient $J^{-1}(\mu) / G$ by applying the Coisotropic Reduction Theorem. Obviously, the condition of clean intersection is trivially satisfied.

In reference [25] it is shown that $J^{-1}(\mu) / G$ is diffeomorphic to the cotangent bundle $T^{*}(Q / G)$. Moreover, considering the symplectic structure $\omega_{\mu}$ on $J^{-1}(\mu) / G$ given by the Marsden-Weinstein reduction procedure, the two manifolds are symplectomorphic, where on $T^{*}(Q / G)$ we are considering the symplectic structure given by the canonical one plus a magnetic term $\omega_{Q / G}+B_{\mu}$ see (Appendix B). Combining the last two paragraphs we can see $\pi(L)$ as a lagrangian submanifold of a cotangent bundle with a modified symplectic structure. We 
proceed now to sketch the aforementioned identification using a connection. Recall that $\pi_{G}: Q \rightarrow Q / G$ is a $G$-principal fiber bundle with the structure group $G$. A connection $A$ on $\pi_{G}: Q \rightarrow Q / G$ induces a splitting

$$
T^{*} Q / G \equiv T^{*}(Q / G) \times_{Q / G} \tilde{\mathfrak{g}}^{*}
$$

(see [25] for a detailed discussion of this splitting) where $\tilde{\mathfrak{g}}^{*}$ denotes the adjoint bundle to $\pi_{Q}: Q \rightarrow Q / G$ via the Coadjoint representation, $\tilde{\mathfrak{g}}^{*}=Q \times_{G} \mathfrak{g}^{*}$ (see [25] and Appendix A for a description of this bundle). The identification (2) is given by

$$
\begin{aligned}
\Psi: T^{*} Q / G & \longrightarrow T^{*}(Q / G) \times_{Q / G} \tilde{\mathfrak{g}}^{*} \\
{\left[\alpha_{q}\right] } & \rightarrow \Psi\left(\left[\alpha_{q}\right]\right)=\left[\left(\alpha_{q} \circ \mathbf{h}, J\left(\alpha_{q}\right)\right)\right],
\end{aligned}
$$

where $\mathbf{h}$ represents the horizontal lift $T_{\pi_{G}(q)}(Q / G) \rightarrow T_{q} Q$ of the connection $A$. Therefore, we have

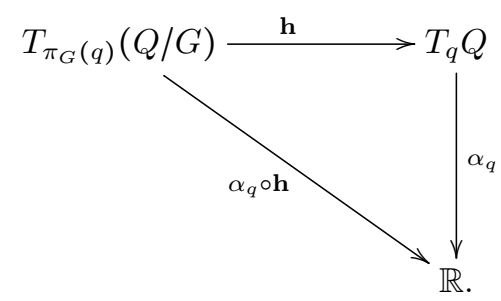

If $\alpha_{q} \in J^{-1}(\mu)$ then $J\left(\alpha_{q}\right)=\mu$, and $\Psi\left(\left[\alpha_{q}\right]\right)=\left(\alpha_{q} \circ \mathbf{h}, J\left(\alpha_{q}\right)=\mu\right)$, so that $J^{-1}(\mu) / G$ can be identified with $T^{*}(Q / G)$.

$$
\Psi\left(J^{-1}(\mu) / G\right)=T^{*}(Q / G) \times_{Q / G}(Q \times\{\mu\} / G) \equiv T^{*}(Q / G)
$$

Remark 7 Notice that $\operatorname{dim}(Q)=n$, and then $\operatorname{dim}\left(J^{-1}(\mu)\right)=2 m-k$ where $\operatorname{dim}(G)=k$. Thus, $\operatorname{dim}\left(J^{-1}(\mu) / G\right)=2 n-k-k=2(n-k)$ and $\operatorname{dim}\left(T^{*}(Q / G)\right)=$ $2(n-k)$.

Notice that $J^{-1}(\mu) / G$ and $T^{*}(Q / G)$ are not only diffeomorphic, moreover, it is possible to show that they are symplectomorphic, while $J^{-1}(\mu) / G$ is considered as a symplectic leaf of $T^{*} Q / G$ and $T^{*}(Q / G)$ is equipped with the canonical symplectic structure modified by a magnetic term (it is explained in the cited paper, [25], and the magnetic term $\beta_{\mu}$ comes from the connection $A, \omega_{Q / G}+\beta_{\mu}$ ). If $\mu=0$, then the magnetic term vanishes and we have the canonical symplectic structure $\omega_{Q / G}$.

Next, we consider a $G$-invariant hamiltonian $h$ on $T^{*} Q$. Then we have

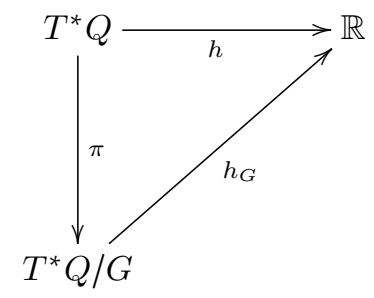


where $h_{G} \circ \pi=h$ is the natural projection of $h$. Consider the mapping $\Psi$ defined above

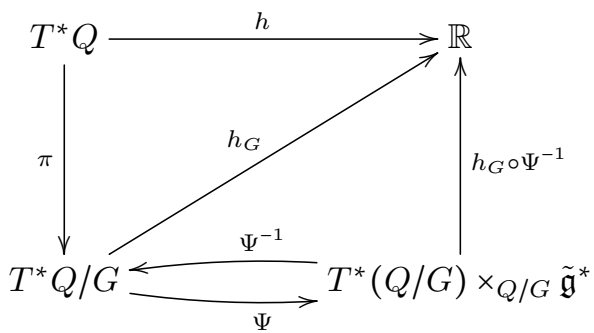

and define $\tilde{h}_{\mu}: T^{*}(Q / G) \longrightarrow \mathbb{R}$ by $\tilde{h}_{\mu}\left(\tilde{\alpha}_{\tilde{q}}\right)=\tilde{h}\left(\tilde{\alpha}_{\tilde{q}},[q, \mu]\right)$, where $\tilde{\alpha}_{\tilde{q}} \in T_{\tilde{q}}^{*}(Q / G)$, $\tilde{q}=[q] \in Q / G, \mu \in \mathfrak{g}^{*}$. Assume that $L$ is $G$-invariant solution of the HamiltonJacobi equation for $h$ and define

$$
\tilde{L}=\Psi(\pi(L)) \subset T^{*}(Q / G) \times_{Q / G} \tilde{\mathfrak{g}}^{*} .
$$

As we have proved before $\tilde{L} \subset T^{*}(Q / G)$ is a lagrangian submanifold with respect to $\omega_{Q / G}+\beta_{\mu}$. Using the previous results we can prove that a $G$-invariant solution for the Hamilton-Jacobi problem for $h$ projects onto a solution of the HamiltonJacobi for $\tilde{h}_{\mu}$. In addition, if $L$ is horizontal then $\tilde{L}$ is horizontal.

Proposition 8 (Reduction) Given L a G-invariant solution of the HamiltonJacobi equation, then $\tilde{L}$ is a solution of the Hamilton-Jacobi equation for $\tilde{h}_{\mu}$ $(\mu=J(L))$. Moreover, if $L$ is horizontal, then $\tilde{L}$ is horizontal.

Proof: Recall that since $L$ is $G$-invariant then $J(L)=\mu$. As we have seen before, $\tilde{L}=\pi(L)$ is a lagrangian submanifold of $J^{-1}(\mu) / G$. Now, we take $\mu \in \mathfrak{g}^{*}$ and since it is a regular value of $J$, then $J^{-1}(\mu)$ is a submanifold of $T^{*} Q$. Since in our case, $L$ is $G$-invariant lagrangian submanifold then $J$ is constant along $L$, say $J(L)=\mu$. Recall that $\mu \in \mathfrak{g}^{*}$ is a fixed point for $A d^{*}$ if and only if $G_{\mu}=G$, and in this case $J^{-1}(\mu)$ is coisotropic. Therefore, we have that $\mu$ is such that $G_{\mu}=G$. This happens for instance if $G$ is abelian. $\tilde{L}=\pi(L)$ is a lagrangian submanifold of $J^{-1}(\mu) / G$, but this is a symplectic leaf with symplectic structure $\omega_{Q / G}+\beta_{\mu}$, when we are using the natural identification via $\Psi$ and considering a fixed connection $A$ in $Q \rightarrow Q / G$ to obtain the corresponding decomposition. In addition, if $\tilde{\alpha}_{\tilde{q}} \in \tilde{L}$, then $\tilde{h}_{\mu}\left(\tilde{\alpha}_{\tilde{q}}\right)=h\left(\Psi^{-1}\left(\tilde{\alpha}_{\tilde{q}}, \mu\right)\right)$. Therefore, $\tilde{h}_{\mu}$ is constant along $\tilde{L}$. Assume now that $L$ is horizontal, so $L=\operatorname{Im}(\gamma)$, for a 1 -form $\gamma$ on $Q$ such that $d \gamma=0$. Since $\gamma$ takes values into $J^{-1}(\mu)$ and is $G$-invariant, then $\gamma$ induces a mapping

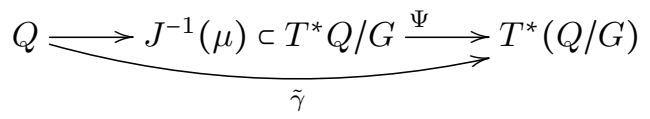

which is $G$-invariant. So it induces a new mapping $\tilde{\gamma}_{\mu}: Q / G \rightarrow T^{*}(Q / G)$ such that $\operatorname{Im}\left(\tilde{\gamma}_{\mu}\right)=\tilde{L}$. 
We also prove a reconstruction theorem. With this theorem at hand, once a reduced solution is found it can be lifted to find a solution of the original unreduced problem.

Proposition 9 (Reconstruction) Assume that $\tilde{L}$ is a lagrangian submanifold of $\left(T^{*}(Q / G), \omega_{Q / G}+\beta_{\mu}\right)$ for some $\mu \in \mathfrak{g}^{*}$ wich is a fixed point of the Coadjoint action. Assume that $\tilde{h}_{\mu}$ is the reduced hamiltonian defined as above and that $\tilde{L}$ is a Hamilton-Jacobi solution for $\tilde{h}_{\mu}$. Using the diffeomorphism

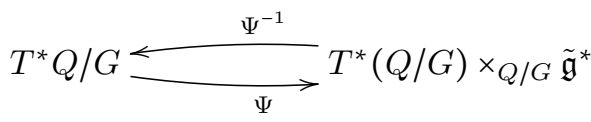

we define $\hat{L}$ by

$$
\hat{L}=\left\{\left(\tilde{\alpha}_{\tilde{q}},[\mu] \tilde{q}\right) \in T^{*}(Q / G) \times_{Q / G} \tilde{\mathfrak{g}}^{*} \text { such that } \tilde{\alpha}_{\tilde{q}} \in \tilde{L}\right\}
$$

and take

$$
L=\pi^{-1}(\hat{L})
$$

Then

1. $L$ is $G$-invariant and lagrangian with respect to the canonical symplectic structure of the cotangent bundle, $\omega_{Q}$, and a solution for the HamiltonJacobi problem given by $h$.

2. If $\tilde{h}$ is horizontal, then $L$ is horizontal too.

Proof: Since $\tilde{L}$ is a Lagrangian submanifold of $\left(T^{*}(Q / G), \omega_{Q / G}+\beta_{\mu}\right)$ and $\Psi_{\mid J^{-1}(\mu) / G}$ is a symplectomorphism, then $\bar{L}=\Psi^{-1}(\hat{L})$ is a lagrangian submanifold of the symplectic leaf $J^{-1}(\mu) / G$. Since $\pi: T^{*} Q \rightarrow T^{*} Q / G$ is a submersion then $\pi^{-1}(\bar{L})$ is an immersed submanifold of dimension $\operatorname{dim}\left(\pi^{-1}(\bar{L})\right)=$ $\operatorname{dim}(\bar{L})+\operatorname{dim}(G)$, and since

$$
\begin{aligned}
\operatorname{dim}(\bar{L}) & =\operatorname{dim}(\tilde{L})=1 / 2 \cdot \operatorname{dim}\left(T^{*}(Q / G)\right) \\
& =1 / 2 \cdot 2 \cdot(\operatorname{dim}(Q)-\operatorname{dim}(G))=\operatorname{dim}(Q)-\operatorname{dim}(G),
\end{aligned}
$$

then $\operatorname{dim}\left(\pi^{-1}(\bar{L})\right)=(\operatorname{dim}(Q)-\operatorname{dim}(G))+\operatorname{dim}(G)=\operatorname{dim}(Q)$ which is half the dimension of $T^{*} Q$; so we only have to show that $\pi^{-1}(\bar{L})$ is an isotropic submanifold. Notice that since $J^{-1}(\mu) / G_{\mu}=J^{-1}(\mu) / G$, then the symplectic structure on $J^{-1}(\mu) / G$ (denoted by $\omega_{\mu}$ ) is the one obtained by the Marsden-Weinstein reduction theorem, which is characterized by the equation $i_{\mu}^{*} \omega_{Q}=\pi^{*} \omega_{\mu}$ where $i_{\mu}: J^{-1}(\mu) \rightarrow T^{*} Q$ is the inclusion and $\omega_{Q}$ the canonical symplectic structure on $T^{*} Q$. Since $\pi^{-1}(\bar{L}) \subset J^{-1}(\mu)$, it is easy to see that

$$
\left(\omega_{Q}\right)_{\mid \pi^{-1}(\bar{L})}=\left(\pi^{*} \omega_{\mu}\right)_{\mid \pi^{-1}(\bar{L})}=0,
$$


and we can conclude that $\pi^{-1}(\bar{L})$ is a lagrangian submanifold. The fact that $h_{\mid \pi^{-1}(\bar{L})}=E$, where $E$ is a constant, follows from the identity

$$
h \mid \pi^{-1}(\bar{L})=\left(\tilde{h}_{\mu}\right)_{\mid \tilde{L}}
$$

and thus the result holds.

Remark 8 It is clear that, by Propositions 8 and 9 , we have a bijection between $G$-invariant solutions of Hamilton-Jacobi problem for $h$ and solutions of the Hamilton-Jacobi equation for $\tilde{h}_{\mu}$ where $\mu$ is a fixed point of the Coadjoint action.

$\{$ G-invariant solutions of $\mathrm{HJ}\} \stackrel{\text { one to one }}{\longrightarrow}\{$ reduced solutions of $\mathrm{HJ}\}$

Remark 9 In the symplectic manifold $\left(T^{*} Q, \omega_{Q}\right)$, given a 1-form $\gamma$ on $Q$ its image is an horizontal lagrangian submanifold if and only if $d \gamma=0$. In that case that lagrangian submanifold is locally given by a generating function $L=\operatorname{Im}(d S)$. Given the symplectic manifold $\left(T^{*}(Q / G), \omega_{Q / G}+\beta_{\mu}\right)$ it is natural to ask which is the analogous condition to $d \gamma=0$. In [25] one can check that $B_{\mu}$ is actually the pullback of a 2 -form on the base $Q / G$ so $B_{\mu}=\pi_{Q / G}^{*} \beta_{\mu}$. So given a 1 -form on $Q / G$, say $\gamma$, its image is lagrangian for the modified structure if and only if $0=\gamma^{*}\left(\omega_{Q / G}+\pi_{Q / G}^{*} \beta_{\mu}\right)=d \gamma+\beta_{\mu}$ or equivalently $d \gamma=-\beta_{\mu}$. In that case, it is no possible in general to find a generating function, and instead one PDE, we have a system of algebraic-PDE equations.

\section{Reduction of $\mathrm{H}-\mathrm{J}$ equation and reduction of dynamics}

Assume that we have a hamiltonian system $\left(T^{*} Q, \omega_{Q}, h\right)$ and let $\gamma$ be a 1form which is a solution of the Hamilton-Jacobi equation for $h$. Then we can construct the projected vector field $X_{h}^{\gamma}$ by

$$
X_{h}^{\gamma}=T \pi_{Q} \circ X_{h} \circ \gamma .
$$

A basic result in the Hamilton-Jacobi theory (see [1]) is that $X_{h}^{\gamma}$ and $X_{h}$ are $\gamma$-related. If we assume that we are in the conditions of the previous sections, that is, we have a free and proper action $\Phi: G \times Q \rightarrow Q$ and all the constructions previously introduced follow, we get a new (reduced) hamiltonian system $\left(T^{*}(Q / G), \omega_{Q / G}+B_{\mu}, \tilde{h}_{\mu}\right)$ and a solution $\tilde{\gamma}$ of the corresponding HamiltonJacobi theory. As before, we can define the projected vector field for the reduced system

$$
X_{\tilde{h}_{\mu}}^{\tilde{\gamma}}=T \pi_{Q} \circ X_{\tilde{h}_{\mu}} \circ \tilde{\gamma} .
$$


and therefore the current situation is the one described in the diagram below

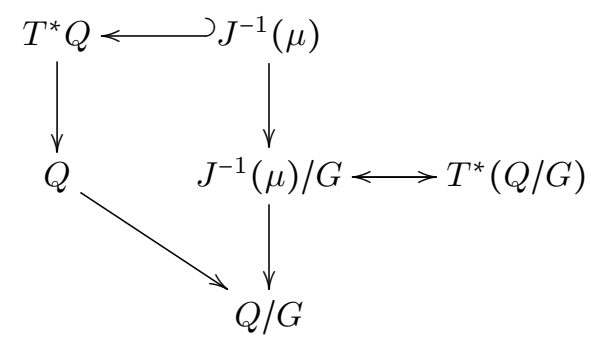

We point out the vector fields and the manifolsd on which they are defined:

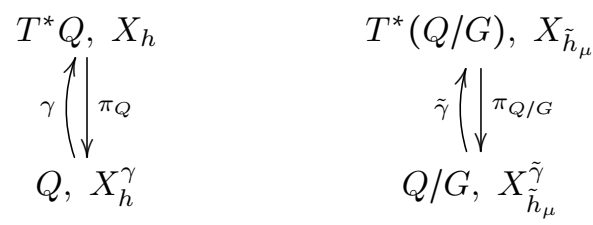

The relation between the dynamics on $T^{*} Q$ and $J^{-1}(\mu) / G$ (recall that we are identifying this space with $\left.T^{*}(Q / G)\right)$ is well-known. There are reconstruction procedures to integrate the vector field $X_{h}$ after integrating the vector field $X_{\tilde{h}_{\mu}}$. So we have

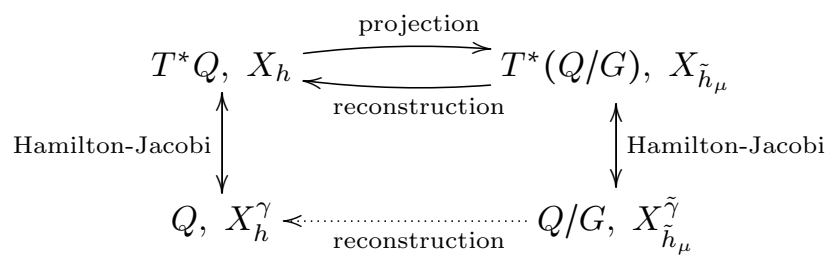

Figure 1: Relations between vector fields

Moreover, since $\tilde{\gamma} \circ \pi_{G}=\pi \circ \gamma$ we can conclude that the vector field $X_{h}^{\gamma}$ projects onto $X_{\tilde{h}_{\mu}}^{\tilde{\gamma}}$ via $\pi_{G}$, and so, $X_{h}^{\gamma}$ is $G$-invariant.

We recall now the basic reconstruction procedure to integrate the vector field $X_{h}^{\gamma}$ via the integration of $X_{\tilde{h}_{\mu}}^{\tilde{\gamma}}$ in order to complete the diagram in Figure 1. Let $c:(a, b) \subset \mathbb{R} \rightarrow Q / G$ be an integral curve of $X_{\tilde{h}_{\mu}}^{\tilde{\gamma}}$ and consider a curve $d(t):(a, b) \rightarrow Q$ such that $\pi_{Q / G} \circ d=c$; for instance, since we made the previous constructions using a connection on the principal bunle $\pi_{G}: Q \rightarrow Q / G$ then $d$ can be taken as the horizontal lift of $c$. Next, consider the connection 1-form, that we will denote also by $A: T Q \rightarrow \mathfrak{g}$, and assume that we have a curve $g:(a, b) \rightarrow G$ such that $\frac{d}{d t} g(t)=A\left(X_{h}^{\gamma}(d(t))-\frac{d}{d t} d(t)\right)$ where we are using the identification $T G \equiv G \times \mathfrak{g}$ given by the left trivialization. It is easy to check that then $g(t) \cdot d(t)$ is an integral curve of $X_{h}^{\gamma}$. 


\section{$5 \quad$ Examples}

It is our believe that the theory above described has wide applicability in concrete situations. Here we present some examples but we would like to stress that much more involved settings fall in our setting.

\section{$5.1 \quad$ Lie groups}

Let $G$ be a Lie group and $T^{*} G$ its cotangent bundle. Using left trivialization we have the identification $T^{*} G \cong G \times \mathfrak{g}^{*}$. Since $T^{*} G / G \cong G \times \mathfrak{g}^{*} / G \cong \mathfrak{g}^{*}$ then, to find a $G$-invariant solution $L$ of the Hamilton-Jacobi problem is equivalent to finding an element $\mu \in \mathfrak{g}^{*}$ such that $A d_{g}^{*}(\mu)=\mu$ for all $g \in G$. Given such $\mu$ we can construct $L \subset G \times \mathfrak{g}^{*}$ given by $L=G \times\{\mu\}$. It is easy to see that a 1 -form defined in this way is closed, $G$-invariant and satisfies $H_{\mid G \times\{\mu\}}=\tilde{H}(\mu)$. Therefore we obtain a characterization of the closed $G$-invariant 1 -forms on a Lie group.

\subsection{The trivial case: $Q=M \times G$}

Assume now that we have $Q=M \times G$ and we are considering the action

$$
\begin{aligned}
\Phi: G \times(M \times G) & \rightarrow(M \times G) \\
(g,(m, h)) & \rightarrow(m, g \cdot h) .
\end{aligned}
$$

If we trivialize $T^{*} G=G \times \mathfrak{g}^{*}$ via the left action, then the lifted action, $\Phi^{T^{*}}$ is given by

$$
\begin{aligned}
& \Phi^{T^{*}}: \quad G \times\left(T^{*} M \times G \times \mathfrak{g}^{*}\right) \longrightarrow\left(T^{*} M \times G \times \mathfrak{g}^{*}\right) \\
& \left(g,\left(\alpha_{m}, h, \mu\right)\right) \quad \rightarrow \quad\left(\alpha_{m}, g \cdot h, \mu\right) .
\end{aligned}
$$

The momentum map is given by $J\left(\alpha_{m}, g, \mu\right)=A d_{g^{-1}}^{*} \mu$. If we have the hamiltonian system $\left(T^{*}(M \times G), H, \Omega_{M \times G}\right)$ (with $H$ assumed $G$-invariant), given $\mu$ such that $G_{\mu}=G$ then $J^{-1}(\mu) / G \cong T^{*} M$ and $\tilde{H}_{\mu}\left(\alpha_{m}\right)=H(\alpha, g, \mu)$ where by the $G$-invariance of $H$ the element $g$ is arbitrary. In this case, the reduced system is equivalent to the hamiltonian system given by $\left(T^{*} M, \tilde{H}_{\mu}, \Omega_{M}\right)$. Assume that $S_{M}: T^{*} M \rightarrow \mathbb{R}$ is the generating function of $\tilde{L}$, a horizontal lagrangian submanifold which solves the Hamilton-Jacobi problem. On the other hand it is easy to see that $\mu$ viewed as a section of the projection onto $G, G \times \mathfrak{g}^{*} \rightarrow G$, is a closed 1-form and so there exists $S_{G}: G \rightarrow \mathbb{R}$ such that $\operatorname{Im}\left(d S_{G}\right)=(g, \mu)$. Let us denote by $S_{M \times G}$ the generating function of the corresponding lagrangian submanifold $L$ obtained by reconstruction from $\tilde{L}$, then we have.

Lemma 10 The generating functions are related by

$$
S_{M \times G}=S_{M}+S_{G}+c
$$

where $c$ is a constant on each connected component. 
Proof: Given $\xi \in \mathfrak{g}$, since $\operatorname{Im}\left(d S_{M \times G}\right) \subset J^{-1}(\mu)$ then $d S\left(\xi_{M \times Q}\right)=\mu(\xi)=$ $d\left(S_{M}+S_{G}\right)\left(\xi_{M \times Q}\right)=d S_{G}\left(\xi_{M \times Q}\right)$. Given $X \in T_{m} M$ the analogous computations holds and the result follows.

\subsubsection{Time-dependent H-J solution for time-independent systems}

An immediate application of the previous result is the obtainment of the classical relation between time-dependent and time independent solutions of the Hamilton-Jacobi equation. This is a very classical ansatz that follows from our results.

Let be $H: T^{*} Q \rightarrow \mathbb{R}$ and consider the corresponding hamiltonian $H^{\mathbb{R}}=$ $H \circ p_{T^{*} Q}+e: T^{*}(\mathbb{R} \times Q) \rightarrow \mathbb{R}$, where $p_{T^{*} Q}: T^{*}(\mathbb{R} \times Q) \rightarrow T^{*} Q$ is the projection onto $T^{*} Q$ and $e$ denotes the time conjugate momentum. We can introduce the action given by translation in time

$$
\begin{aligned}
& \Phi: \quad \mathbb{R} \times(\mathbb{R} \times Q) \longrightarrow(\mathbb{R} \times Q) \\
& (r,(t, q)) \rightarrow(t+r, q) .
\end{aligned}
$$

The corresponding lifted action is

$$
\begin{aligned}
\Phi^{T^{*}}: \mathbb{R} \times T^{*}(\mathbb{R} \times Q) & \longrightarrow T^{*}(\mathbb{R} \times Q) \\
\left(r,\left(t, e, \alpha_{q}\right)\right) & \rightarrow\left(t+r, e, \alpha_{q}\right) .
\end{aligned}
$$

The momentum map is just

$$
J\left(t, e, \alpha_{q}\right)=e .
$$

If $E \in \mathbb{R} \cong \mathbb{R}^{*}$ then $\mathbb{R}_{E}=\mathbb{R}$ since the group is abelian and $J^{-1}(E) \cong \mathbb{R} \times T^{*} Q$ and $\mathbb{R} \times T^{*} Q / \mathbb{R} \cong T^{*} Q$. Summarizing, we have that $\left(J^{-1}(E) / G, \tilde{H}_{E}^{\mathbb{R}}, \tilde{\Omega}\right)$ is given by $\left(T^{*} Q, H, \Omega_{Q}\right)$ and, if we denote by $S$ the generating function of $L$ and by $W$ the generating function of $\tilde{L}$, then we obtain $S_{\mathbb{R}}=t \cdot E$ and $S_{Q}=W$ and we recover

$$
S=t \cdot E+W
$$

\subsection{Complete Solutions}

This subsection is devoted to applying the previous results to what is usually called a complete solution of the Hamilton-Jacobi equation. The knowledge of a complete solution of the Hamilton-Jacobi equation is equivalent to integrating the Hamilton's equations of motion (see [3]). Before getting into our results, we sketch in this subsection the classical results. They are local and written in a coordinate dependent way, but the global, geometric aspects of the theory are easier to understand after taking a look at the classical theory. We restrict ourselves to the time-independent case but the results can be easily extended to the time-dependent setting.

Let $h\left(q^{i}, p_{i}\right)$ be a hamiltonian on the phase space $\left(q^{i}, p_{i}\right), i=1, \ldots, n$. By a complete solution of the Hamilton-Jacobi equation for $h$ we mean the following. 
Definition 11 A complete solution of the Hamilton-Jacobi equation for the hamiltonian $h\left(q^{i}, p_{i}\right), i=1, \ldots, n$ is a real-valued function $S\left(t, q^{i}, \alpha^{i}\right), i=1, \ldots, n$, such that

1. For every (fixed) value of the parameters $\left(\alpha^{i}\right), S\left(t, q^{i}, \alpha^{i}\right)$ satisfies the Hamilton-Jacobi equation,

$$
\frac{\partial S}{\partial t}+h\left(t, q^{i}, \frac{\partial S}{\partial q^{i}}\left(t, q^{i}\right)\right)=0
$$

2. The non-degeneracy condition: consider the matrix with component $i, j$ given by $\frac{\partial^{2} S}{\partial q^{i} \partial \alpha^{j}}$, that we denote by $\left(\frac{\partial^{2} S}{\partial q^{i} \partial \alpha^{j}}\right)$, then

$$
\operatorname{det}\left(\frac{\partial^{2} S}{\partial q^{i} \partial \alpha^{j}}\right) \neq 0 .
$$

Then, we can define (at least locally by the implicit function theorem) the following implicit, time-dependent transformation, from the $\left(t, q^{i}, p_{i}\right)$-space to the $\left(t, \alpha^{i}, \beta_{i}\right)$-space:

$$
\frac{\partial S}{\partial q^{i}}\left(t, q^{i}, \alpha^{i}\right)=p_{i} \quad-\frac{\partial S}{\partial \alpha^{i}}\left(t, q^{i}, \alpha^{i}\right)=\beta_{i} .
$$

A computation shows that this transformation sends the system to equilibrium, i.e., Hamiltlon's equations become now

$$
\begin{aligned}
& \frac{d \alpha^{i}}{d t}(t)=0, \\
& \frac{d \beta_{i}}{d t}(t)=0,
\end{aligned}
$$

see $[1,3]$.

We give now a geometric interpretation of the previous procedure. The function $S$ can be interpreted as a function on the product manifold $\mathbb{R} \times Q \times Q$ and so $\operatorname{Im}(d S)$ is a lagrangian submanifold in $T^{*}(\mathbb{R} \times Q \times Q)$ (notice that we are thinking about the $\left(q^{i}\right)$ as coordinates on the first $Q$, and $\left(\alpha^{i}\right)$ as coordinates on the second factor $Q)$. On the other hand, consider the projections $\pi_{I}: T^{*}(\mathbb{R} \times$ $Q \times Q) \rightarrow \mathbb{R} \times T^{*} Q, I=1,2$, defined by $\pi_{I}\left(t, e, \alpha^{1}, \alpha^{2}\right)=\left(t,(-1)^{I+1} \alpha^{I}\right)$. With these geometric tools, the non-degeneracy condition is equivalent to saying that $\pi_{I \mid \operatorname{Im}(d S)}$ is a local diffeomorphism for $I=1,2$. We assume here for simplicity that it is a global diffeomorphism, so we can consider the mapping $\pi_{2 \mid \operatorname{Im}(d S)} \circ$ $\left(\pi_{1 \mid \operatorname{Im}(d S)}\right)^{-1}: T^{*}(\mathbb{R} \times Q) \rightarrow T^{*}(\mathbb{R} \times Q)$. This mapping can be easily checked to be the global description of the change of variables introduced above. The Hamilton-Jacobi equation, can be understood as the fact that $d S^{*} h^{e x t}=0$, where $h^{e x t}=\pi_{1}^{*} h+e$. The diagram below helps to have a global picture of the procedure:

In the precedent setting all the information is given by the lagrangian manifold defined by $\operatorname{Im}(d S)$, so we can introduce a generalized solution to the Hamilton-Jacobi equation as follows. 


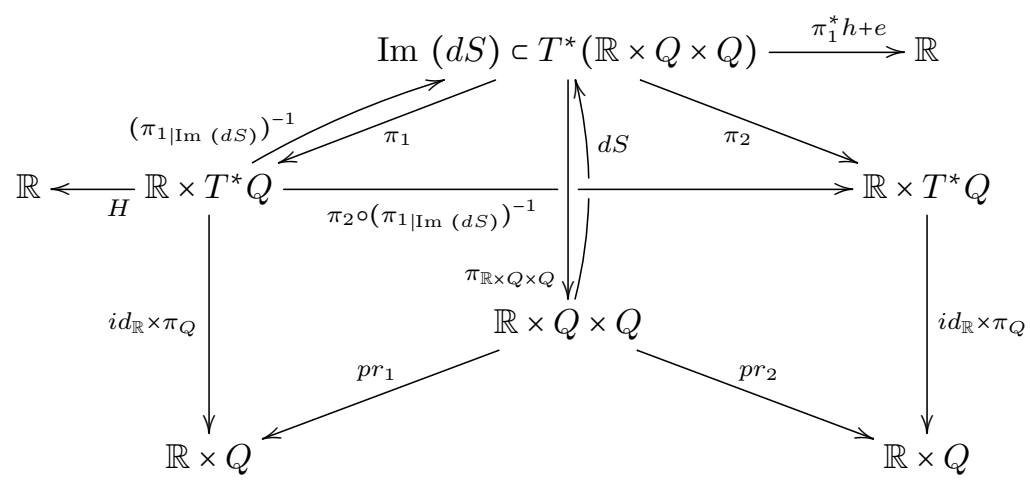

Figure 2: Geometric interpretation of complete solutions of the H-J equation

Definition 12 A lagrangian submanifold $L$ in $T^{*}(\mathbb{R} \times Q \times Q)$ is a complete solution of the Hamilton-Jacobi equation if

1. $L \subset\left(h^{e x t}\right)^{-1}(e)$.

2. The restriction of $\pi_{I}$ to $L$ is a diffeomorphism. From now on we will refer to this property as the non-degeneracy condition.

Remark 10 When $L$ is given by $\operatorname{Im}(d S)$ we say that $S$ is a generating function for the transformation induced by $L$. This type of generating functions are usually called in the literature type I generating functions, see [19]. It is remarkable that our theory deals with the lagrangian submanifolds instead of their generating functions, so our theory is applicable to other types of generating functions. This does not happen in previous approaches to reduction of the Hamilton-Jacobi theory.

Under the previous conditions we are still able to define the symplectomorphism that solves Hamilton's equations. We can now apply our reduction procedure. Assume that we have an action $\Phi: G \times Q \rightarrow Q$, such that $\Phi^{T^{*}}$ leaves the hamiltonian invariant. We consider the diagonal action

$$
\begin{aligned}
\Phi_{0}: \quad G \times \mathbb{R} \times Q \times Q & \rightarrow \mathbb{R} \times Q \times Q \\
\left(g,\left(t, q^{1}, q^{2}\right)\right) & \rightarrow\left(t, \Phi\left(g, q^{1}\right), \Phi\left(g, q^{2}\right)\right) .
\end{aligned}
$$

It is easy to see that $\Phi_{0}^{T^{*}}$ leaves $h^{\text {ext }}$ invariant and the corresponding momentum mapping is $J_{0}=J \circ \pi_{1}-J \circ \pi_{2}$, where $J$ is the momentum mapping corresponding to the action $\Phi$. Then, we can look for $G$-invariant complete solutions. After applying our reduction method, we obtain the (reduced) cotangent manifold $T^{*}\left(\mathbb{R} \times \frac{Q \times Q}{G}\right)$. All the reduction theory for Hamilton-Jacobi applies in this manner to complete solutions in a straightforward way. 
Remark 11 A simple computation, following the arrows in Figure 2, shows that $G$-invariant lagrangian submanifolds in $T^{*}(\mathbb{R} \times Q \times Q)$ which satisfy the non-degeneracy condition induce time-dependent $G$-equivariant symplectic automorphisms on $T^{*} Q$.

Remark 12 If our hamiltonian comes from a regular lagrangian, $\mathcal{L}$, then there is always a (local) $G$-invariant solution which lives in $J_{0}^{-1}(0)$, just the one given by the action functional

$$
S(t, q, \bar{q})=\int_{c} \mathcal{L}(\dot{c}) d t
$$

where $c:[a, b] \rightarrow Q$ is the curve satisfying the Euler-Lagrange equations and verifying $c(a)=q$ and $c(b)=\bar{q}$. Under the previous assumptions, that curve exists for $q$ and $\bar{q}$ close enough.

Remark 13 There is a very important lagrangian submanifold, the one given by the flow. Let $\Psi_{t}^{h}$ be the flow of the hamiltonian vector field $X_{h}$. Then we have the lagrangian submanifold in $T^{*}(\mathbb{R} \times Q \times Q)$

$$
L=\left\{\left(t, h\left(t, \alpha_{q}\right), \alpha_{q},-\Psi_{t}^{h}\left(\alpha_{q}\right)\right) \text { such that } t \in \mathbb{R}, \alpha_{q} \in T^{*} Q\right\}
$$

At the end, the Hamilton-Jacobi theory is about finding a generating function for this lagrangian submanifold. If $G$ is a symmetry of the hamiltonian then $L$ is $G$-invariant and lives in the 0-level set, as a consequence of the conservation of the momentum mapping. This lagrangian submanifold can locally be obtained by type II generating function (see next section).

Remark 14 Observe that $T^{*}\left(\mathbb{R} \times \frac{Q \times Q}{G}\right)$ has a well-known geometric structure; indeed, it is the cotangent bundle of the gauge groupoid $\mathbb{R} \times \frac{Q \times Q}{G}$. It suggest that the geometric structure behind all this theory is the symplectic groupoid structure. Moreover, following this pattern we were able to develop a HamiltonJacobi theory for certain Poisson manifolds that will appear in a forthcomming paper [14].

Remark 15 The reduced lagrangian submanfold $\hat{L} \subset T^{*}\left(\mathbb{R} \times \frac{Q \times Q}{G}\right)$ induces a (Poisson) transformation $\mathbb{R} \times T^{*} Q / G \rightarrow \mathbb{R} \times T^{*} Q / G$, using the source and the target of the groupoid structure, in the same way we have used the projections $\pi_{I}$ above. The Poisson structure considered on $\mathbb{R} \times T^{*} Q / G$ is the product of the 0 Poisson structure on $\mathbb{R}$ and the natural Poisson structure induced on $T^{*} Q / G$ by the quotient of the symplectic structure on $T^{*} Q$. In the case $Q=G$, the source and the target are the left and right momentum mappings $J^{L}$ and $J^{R}$, and in the pair groupoid case the projections $\pi_{I}$. This reinforces the idea that symplectic groupoids play an essential role in this theory.

Remark 16 As a by-product, we obtain all the results related to the reduction of the Hamilton-Jacobi theory of reference [18]. The previous discussion specializes to Lie groups, $Q=G$ and then the reduced space $T^{*}\left(\mathbb{R} \times \frac{Q \times Q}{G}\right)=T^{*}\left(\mathbb{R} \times \frac{G \times G}{G}\right)$ can be identified with $T^{*}(\mathbb{R} \times G)$ to recover the theory in Ge and Marsden, [18]. 


\subsubsection{Other types of generating function}

The goal of this section is to show how our results can be applied to other types of generating functions; in this way we recover some classical results about cyclic coordinates. We chose the so-called type II generating functions, but since our theory is valid for any lagrangian submanifold it can be used to deal with any type of generating functions. These type II generating functions are very important because they can generate the identity transformation and all the "nearby" canonical transformations. We introduce below the classical situation, we assume that $Q=\mathbb{R}^{n}$ and so $T^{*} Q=\mathbb{R}^{2 n}$ and consider global coordinates $\left(q^{i}, p_{i}\right), i=1, \ldots, n$. Doubling these coordinates we get a coordinate system for $T^{*}(Q \times Q)=\mathbb{R}^{4 n}$, say $\left(q^{i}, p_{i}, \alpha^{i}, \beta_{i}\right)$, and we obtain coordinates $\left(t, e, q^{i}, p_{i}, \alpha^{i}, \beta_{i}\right)$ on $T^{*}(\mathbb{R} \times Q \times Q)$. Given a function $S\left(t, q^{i}, \beta_{i}\right)$ it is easy to check that the submanifold given by

$L=\left\{\left(t, \frac{\partial S}{\partial t}\left(t, q^{i}, \beta_{i}\right), q^{i}, \frac{\partial S}{\partial q^{i}}\left(t, q^{i}, \beta_{i}\right), \frac{\partial S}{\partial \beta_{i}}\left(t, q^{i}, \beta_{i}\right),-\beta_{i}\right)\right.$ such that $\left.t, q^{i}, \beta_{i} \in \mathbb{R}\right\}$

is lagrangian.

Remark 17 A more detailed explanation about the construction of this submanifold can be found in [14].

Following the same pattern than above, such generating function gives a time-dependent canonical transformation, given implicitly by

$$
\frac{\partial S}{\partial q^{i}}\left(t, q^{i}, \beta_{i}\right)=p_{i}, \quad \frac{\partial S}{\partial \beta_{i}}\left(t, q^{i}, \beta_{i}\right)=\alpha^{i} .
$$

as long as $\operatorname{det}\left(\frac{\partial^{2} S}{\partial q^{i} \partial \beta_{j}}\right) \neq 0$.

Now, our reduction procedure can be applied to the lagrangian submanifold $L$ in a straightforward way. We work out here the details in the case of a time-independent hamiltonian with one cyclic variable in order to recover some results present in the literature, the cases with more than one cyclic variables are obvious. Assume that $h\left(q^{i}, p_{i}\right)$ does not depend on $t$ and $q^{1}$, i.e. $q^{1}$ is a cyclic variable. We are looking for a type II solution of the Hamilton-Jacobi equation for $h$, that is, $S\left(t, q^{i}, \beta_{i}\right)$ such that

1. The Hamilton-Jacobi equation $\frac{\partial S}{\partial t}+h\left(q^{i}, \frac{\partial S}{\partial q^{i}}\right)=E$, where $E$ is a real constant.

2. Non-degeneracy condition, $\operatorname{det}\left(\frac{\partial^{2} S}{\partial q^{i} \partial \beta_{j}}\right) \neq 0$.

Using Section 5.2.1 we assume $S\left(t, q^{i}, \beta_{i}\right)=t \cdot E+W\left(q^{i}, \beta_{i}\right)$, where $W$ should satisfy

$$
h\left(q^{i}, \frac{\partial W}{\partial q^{i}}\left(q^{i}, \beta_{i}\right)\right)=F
$$


for some constant $F$ and the non-degeneracy condition. Notice that such function $W$ gives a lagrangian submanifold in $\mathbb{R}^{4 n}$ by

$$
L_{1}=\left\{\left(q^{i}, \frac{\partial W}{\partial q^{i}}\left(q^{i}, \beta_{i}\right), \frac{\partial W}{\partial \beta_{i}}\left(q^{i}, \beta_{i}\right),-\beta_{i}\right) \text { such that } q^{i}, \beta_{i} \in \mathbb{R}\right\} .
$$

In order to solve (6) we use the theory previouly developed. Notice that $q^{1}$ is a cyclic variable if and only if the hamiltonian is invariant by the $\mathbb{R}$ action given by $\left(r,\left(q^{1}, \ldots, q^{i}, \ldots, q^{n}\right)\right)=\left(q^{1}+r, \ldots, q^{i}, \ldots, q^{n}\right)$, which has an associated momentum mapping given by $J\left(q^{i}, p_{i}\right)=p_{1}$. The corresponding diagonal action is given by

$$
\left.\left(r,\left(q^{i}, p_{i}, \alpha^{i}, \beta_{i}\right)\right)=\left(q^{1}+r, \ldots, q^{i}, \ldots, q^{n}, p_{i}, \alpha^{1}+r, \ldots, \alpha^{i}, \ldots, \alpha^{n}, \beta_{i}\right)\right)
$$

with momentum mapping

$$
J\left(q^{i}, p_{i}, \alpha^{i}, \beta_{i}\right)=p_{1}+\beta_{1} .
$$

So, if we are looking for a lagrangian submanifold $L_{1}$ living in the 0 level set of the momentum mapping, that is natural regarding the previous remarks, we should impose

$$
\frac{\partial W}{\partial q^{1}}-\beta_{1}=0
$$

which implies, by simple integration, that

$$
W=q^{1} \beta_{1}+V\left(q^{i}, \beta_{j}\right), \quad i=2, \ldots, n ; j=1, \ldots, n,
$$

where the important observation here is that $V$ does not depend on the cyclic variable $q^{1}$. In this way, we have reduced the number of independent variables by one, this could simplify drastically the Hamilton-Jacobi equation. Here we have recovered the classical ansantz for cyclic variables, see [2, 19], from our geometric interpretation of the Hamilton-Jacobi theory in a straightforward way.

Remark 18 In the case of more than one cyclic variables an analogous result holds, there

$$
W=q^{l} \beta_{l}+V\left(q^{i}, \beta_{j}\right), \quad i=k, \ldots, n ; j=1, \ldots, n,
$$

where $l=1, \ldots k$ are the cyclic variables.

We show how to obtain a complete solution, using this method, of the Hamilton-Jacobi equation for a heavy-top with two equal moments of inertia. The hamiltonian is given by

$$
h\left(\theta, \phi, \psi, p_{\theta}, p_{\phi}, p_{\psi},\right)=1 / 2\left(\frac{p_{\theta}^{2}}{I}+\frac{\left(p_{\phi}-p_{\psi} \cos (\theta)\right)^{2}}{I \sin ^{2}(\theta)}+\frac{p_{\psi}^{2}}{J}\right)+m g l \cos (\theta),
$$

where $I, J$ are the moments of inertia, $m$ the mass, $g$ the acceleration of gravity. Using the constructions above

$$
S=t \cdot E+W\left(\theta, \phi, \psi, \beta_{1}, \beta_{2}, \beta_{3}\right)
$$


and

$$
W\left(\theta, \phi, \psi, \beta_{1}, \beta_{2}, \beta_{3}\right)=\phi \cdot \beta_{2}+\psi \cdot \beta_{3}+V\left(\theta, \beta_{1}, \beta_{2}, \beta_{3}\right) .
$$

Taking into account all this expressions, we get for the Hamilton-Jacobi equation

$$
1 / 2\left(\frac{\partial V^{2}}{\partial \theta} \frac{1}{I}+\frac{\left(\beta_{2}-\beta_{3} \cos (\theta)\right)^{2}}{I \sin ^{2}(\theta)}+\frac{\beta_{3}^{2}}{J}\right)+m g l \cos (\theta)=F .
$$

From here it is immediate to integrate the equation and to choose a solution that is non-degenerate; notice that the only unknown is $\frac{\partial V}{\partial \theta}$ and so by simple integration we can achieve the solution. Although this result was well-known classically, our point here is that it fits directly within our setting. Compare our results with [2], p. 315.

\subsection{Calogero-Moser system}

We would like to treat another concrete application. Here we deal with a Calogero-Moser system of two particles. Although simple, this system illustrates how our method works. Our aim here is to show that solutions in $J^{-1}(0)$

correspond to self-similar solutions, while solutions for different values of the momentum mapping give solutions no easlily obtained by other means. Consider the hamiltonian

$$
\begin{aligned}
H: \quad T^{*} \mathbb{R}^{2} & \rightarrow \mathbb{R} \\
\left(q^{1}, q^{2}, p_{1}, p_{2}\right) & \rightarrow H\left(q^{1}, q^{2}, p_{1}, p_{2}\right)=1 / 2\left(p_{1}^{2}+p_{2}^{2}\right)+1 /\left(q^{1}-q^{2}\right)^{2} .
\end{aligned}
$$

In this example $Q=\mathbb{R}^{2}$ and the action

$$
\begin{array}{rlll}
\Phi: & \mathbb{R} \times \mathbb{R}^{2} & \rightarrow \mathbb{R}^{2} \\
\left(r,\left(q^{1}, q^{2}\right)\right) & \rightarrow & \left(r+q^{1}, r+q^{2}\right)
\end{array}
$$

is a symmetry of the system, i.e., the hamiltonian is invariant under the corresponding lifted action, $\Phi^{T^{*}}$. We are now looking for a solution of the equation

$$
H\left(q^{1}, q^{2}, \frac{\partial W}{\partial q^{1}}, \frac{\partial W}{\partial q^{2}}\right)=E
$$

which in this case becomes

$$
1 / 2\left({\frac{\partial W^{2}}{\partial q^{1}}}^{2}+\frac{\partial W^{2}}{\partial q^{2}}\right)+1 /\left(q^{1}-q^{2}\right)^{2}=0
$$

We are looking for solutions in $J^{-1}(0)$, where

$$
\begin{aligned}
J: \quad T^{*} \mathbb{R}^{2} & \rightarrow \mathbb{R} \\
\left(q^{1}, q^{2}, p_{1}, p_{2}\right) & \rightarrow J\left(q^{1}, q^{2}, p_{1}, p_{2}\right)=p_{1}+p_{2}
\end{aligned}
$$

Then, $J^{-1}(0)=\left\{\left(q^{1}, q^{2}, p_{1}, p_{2}\right)\right.$ such that $\left.p_{2}=-p_{1}\right\}$, and thus coordinates on $J^{-1}(0)$ are given by $\left(q^{1}, q^{2}, p\right) \rightarrow\left(q^{1}, q^{2}, p,-p\right)$. In the same way, $J^{-1}(0) / \mathbb{R}$ is 
$\mathbb{R}^{2}$, with coordinates $(q, p)$ and the natural projection $\pi: J^{-1}(0) \rightarrow J^{-1}(0) / \mathbb{R}$ reads $\pi\left(q^{1}, q^{2}, p\right)=\left(q=q^{1}-q^{2}, p\right)$. Some abuse of notation is made, but there is no room for confusion. Since $H$ is $\mathbb{R}$-invariant there is a reduced hamiltonian, $\bar{H}: J^{-1}(0) / \mathbb{R} \equiv \mathbb{R}^{2} \rightarrow \mathbb{R}$, such that $\bar{H}(q, p)=p^{2}+1 / q^{2}$. Now the reduced Hamilton-Jacobi equation is just an ODE

$$
\bar{H}\left(q, \frac{\partial \bar{W}}{\partial q}\right)=E,
$$

and the reduced Hamilton-Jacobi equation can be integrated looking for a primitive

$$
\bar{W}=\int\left(E-1 / q^{2}\right)^{1 / 2}
$$

for the values of $q$ where it makes sense. That can be checked to be

$$
\bar{W}(q)=\left(\sqrt{E q^{2}-1}-\arctan \left(\frac{1}{\sqrt{E q^{2}-1}}\right)\right) .
$$

Then, the reconstruction procedure gives us

$$
W\left(q^{1}, q^{2}\right)=\bar{W}\left(q^{1}-q^{2}\right)=\sqrt{E\left(q^{1}-q^{2}\right)^{2}-1}+\arctan \left(\frac{1}{\sqrt{E\left(q^{1}-q^{2}\right)^{2}-1}}\right)
$$

which is defined when $\left|q^{1}-q^{2}\right|>1 / \sqrt{E}$.

Remark 19 Notice that these solutions are self-similar solutions, i.e., they depend on variables on the quotient. The solutions are only defined to the values of $q$ defined above due to the fact that the lagrangian submanifolds under study stop being horizontal for the projection $\pi_{Q}$ when they reach the value $1 / \sqrt{E}$.

When the reduction at $J^{-1}(\mu)$ is carried out, similar results are obtained. There, $J^{-1}(\mu)=\left\{\left(q^{1}, q^{2}, p_{1}, p_{2}\right)\right.$ such that $\left.p_{2}=-p_{1}+\mu\right\}$. The projection $J^{1} \mu \rightarrow$ $J^{-1}(\mu) / \mathbb{R}$ is defined in an analogous fashion, and the reduced hamiltonian reads

$$
H(q, p)=1 / 2\left((\mu-p)^{2}+p^{2}\right)+1 / q,
$$

and the Hamilton-Jacobi equation reads

$$
1 / 2\left(\left(\mu-\frac{\bar{W}}{q}\right)^{2}+\frac{\bar{W}^{2}}{q}\right)+1 / q .
$$

Direct integration does not work here, but the reduced Hamilton-Jacobi equation is an ODE easily solvable.

$$
\bar{W}(q)=\mu q+\sqrt{\left(E+\mu^{2}\right) q^{2}-1}+\arctan \left(\frac{1}{\sqrt{\left(E+\mu^{2}\right) x^{2}-1}}\right)
$$

and now the reconstruction proecedure easily gives

$$
W\left(q^{1}, q^{2}\right)=W\left(q^{1}-q^{2}\right)+\mu q^{2} .
$$

Remark 20 Notice that now we obtained something diferent from self-similar solutions. 


\section{Conclusions and Future Research}

In this paper we developed a complete theory of reduction and reconstruction of the Hamilton-Jacobi equation for hamiltonian systems with symmetry. The symmetry is supposed to be the lifted action of an action on the configuration manifold, $Q$. Our theory is explained for the time-independent and time dependent Hamilton-Jacobi equations, moreover, complete solutions are also considered. We showed that our theory unifies and extends previous approaches by Ge and Marsden and we can recover in a straightforward way the classical ansätze used in the literature to deal with cyclic variables and time-independent hamiltonians. The results in [17] are also particular instances of our approach. On the other hand, one of the main points of our theory is that we link reduction theory with symplectic groupoids. That link was started in [16] but our approach is quite different and will appear elsewhere ([14]) with some applications to (Poisson) numerical methods. Some open problem related to this work are:

1. Relate our theory to the theory of generating functions in [28, 29]. The theory developed there relies on generating function, so it seems that our theory should be the natural framework to deal with this kind of theories. Connections with the Poincaré generating function would be also very interesting.

2. Develop an analogous theory for general symmetries. Although quite useful our setting only deals at this moment with cotangent lifts of symmetries, to develop an analogous theory for any kind of symmetries should provide means to integrate more general systems. The results in [13] could be of some help in this regard.

3. Construction of geometric integrators from complete solutions of the Hamilton-Jacobi equation. Complete solutions of the Hamilton-Jacobi equations are sometimes hard to find, but they can be approximated in order to find numerical methods that preserve the underlying geometry. This procedure is well-known in the symplectic case, see $[12,20]$. Our setting is useful in order to develop the analogous Poisson integrators in the situations treated here. Related work will appear in [14].

\section{A Principal bundles and adjoint bundles}

Consider the $G$-principal bundle

$$
\pi: Q \rightarrow Q / G
$$

with the action on the left

$$
\Phi: G \times Q \rightarrow Q
$$

and $F$ a manifold endowed with a left action

$$
\rho: G \times F \rightarrow F .
$$


We shall construct the fiber bundle $Q \times_{G} F$. Let $Q \times F$ be the product manifold and we introduce the action

$$
\begin{aligned}
G \times(Q \times F): & \longrightarrow Q \times F \\
(g,(q, f)) & \rightarrow(\Phi(g, q), \rho(g, f)) .
\end{aligned}
$$

The quotient space of $Q \times F$ by this action is called the the fiber bundle over the base $Q / G$ with standard fiber $F$ and structure group $G$, which is associated with the principal bundle $Q$ and it is denoted by $Q \times_{G} F$. We introduce now the differentiable structure of this bundle. The mapping

$$
\begin{aligned}
& \tilde{\pi}: Q \times F \longrightarrow Q / G \\
& (q, f) \quad \rightarrow \quad \pi(q)
\end{aligned}
$$

induces a mapping $\hat{\pi}: Q \times_{G} F \rightarrow Q / G$. Since for each $x \in Q / G$ there exists a neighborhood $U$ such that $\pi^{-1}(U) \equiv U \times G$, it can be easily seen that there is an isomorphism $\hat{\pi}^{-1}(U) \equiv U \times F$. Therefore we can introduce a differentiable structure on $Q \times_{G} F$ by the requirement that $\hat{\pi}^{-1}(U)$ is an open submanifold of $Q \times_{G} F$ diffeomorphic with $U \times F$ under the isomorphism $\hat{\pi}^{-1}(U) \equiv U \times F$. Then, it follows that $\hat{\pi}$ is a differentiable mapping.

We now specialize the previous construction to the case when $F=\mathfrak{g}^{*}$ and the action is given by

$$
\begin{aligned}
\tilde{\rho}: \quad G \times \mathfrak{g}^{*} & \longrightarrow \mathfrak{g}^{*} \\
(g, \mu) & \rightarrow \tilde{\rho}(g, \mu)=A d_{g^{-1}}^{*}(\mu)
\end{aligned}
$$

The corresponding bundle obtained using the action $\tilde{\rho}$ will be denoted by $\tilde{\mathfrak{g}}^{*}$.

\section{B Magnetic Terms}

Let $Q$ be a manifold, $\Phi: G \times Q \rightarrow Q$ a free and proper action, $\Phi^{T^{*}}$ the cotangentlifted action and $J: T^{*} Q \rightarrow \mathfrak{g}$ the corresponding momentum mapping. Recall that $Q / G$ is endowed with a structure of differentiable manifold and $\pi_{G}: Q \rightarrow$ $Q / G$ is a principal bundle.

We will prove that $J^{-1}(0) / G$ is symplectomorphic to $\left(T^{*}(Q / G), \omega_{Q / G}\right)$. To see that, consider the codifferential of the mapping $\pi_{G}, T \pi_{G}^{*}: T^{*}(Q / G) \rightarrow T^{*} Q$ and compose with the natural projection over the quotient $p: T^{*} Q \rightarrow T^{*} Q / G$. Then the mapping $p \circ T^{*} \pi_{G}$ is easily seen to give the desired identification when restricted to its image, which is $J^{-1}(0) / G$. Notice that although the codifferential $T^{*} \pi_{G}$ is not a mapping (it is multi-valued), the composition does become an identification.

$J^{-1}(\mu) / G_{\mu}$ is known to be symplectomorphic to $\left(T^{*}(Q / G), \omega_{Q / G}+B_{\mu}\right)$ when $G_{\mu}=G$ and where $B_{\mu}$ is a magnetic term. To prove that, take $\alpha_{\mu}$ a 1-form on $Q$ such that

1. $\alpha_{\mu}$ is $G$-invariant by $\Phi^{T^{*}}$. 


\section{2. $J \circ \alpha_{\mu}=\mu$.}

Then we have the shift by $\alpha_{\mu}$ given by shift: $T^{*} Q \rightarrow T^{*} Q$, such that $\operatorname{shift}\left(\alpha_{q}\right)=$ $\alpha_{q}-\alpha_{\mu}$. This mapping is $G$-equivariant and $\operatorname{shift}^{*}\left(\omega_{Q / G}\right)=\omega_{Q / G}-d \alpha_{\mu}$. Moreover, this map satisfies shift $\left(J^{-1}(\mu)\right)=J^{-1}(0)$ and thus, by $G$-equivariance, $\frac{\text { shift }}{G}\left(J^{-1}(\mu) / G\right)=J^{-1}(0) / G$, where $\frac{\text { shift }}{G}$ is the mapping induced on the quotient. The right hand side of the last equality is identified with $T^{*}(Q / G)$ but since shift is not a symplectomorphim between the canonical symplectic structures of cotangent bundles, the form $\omega_{Q / G}$ must be modified. Since $J \circ \alpha_{\mu}=\mu$, then $\alpha_{\mu}\left(\xi_{Q}\right)=\mu(\xi)$ is a constant function on $Q$. We deduce that

$$
i_{\xi_{Q}} d \alpha_{\mu}=\mathcal{L}_{\xi_{Q}} d \alpha_{\mu}-d\left(\alpha_{\mu}\left(\xi_{Q}\right)\right)=0
$$

and so there exists a unique 2 -form on $Q / G$ such that $\pi_{G}^{*} \beta_{\mu}=d \alpha_{\mu}$. It is not hard to see now that $J^{-1}(\mu) / G$ with the symplectic structure provided by the MardenWeinstein reduction is symplectomorphic to the cotangent bundle $T^{*}(Q / G)$ with the symplectic structure given by $\omega_{Q / G}+\pi_{Q / G}^{*} \beta_{\mu}$.

Remark 21 In the constructions of our paper, we used a connection from the beginning. With a connection at hand, the construction of the form $\alpha_{\mu}$ is just the composition of the connection 1-form (which is a $\mathfrak{g}$-valued 1-form) and $\mu$.

\section{Acknowledgments}

This work has been partially supported by MINECO MTM 2013-42-870-P and the ICMAT Severo Ochoa project SEV-2011-0087. M. Vaquero wishes to thank MINECO for a FPI-PhD Position. We wish to thank D. Iglesias-Ponte and J.C. Marrero for several useful discussions.

\section{References}

[1] Abraham, R., And Marsden, J. E. Foundations of mechanics. Benjamin/Cummings Publishing Co., Inc., Advanced Book Program, Reading, Mass., 1978. Second edition, revised and enlarged, With the assistance of Tudor Raţiu and Richard Cushman.

[2] Ardema, M. Analytical Dynamics: Theory and Applications. Kluwer Academic/ Plenum Publishers, New York, 2005.

[3] Arnol'D, V. I. Mathematical methods of classical mechanics, vol. 60 of Graduate Texts in Mathematics. Springer-Verlag, New York, 1993. Translated from the 1974 Russian original by K. Vogtmann and A. Weinstein, Corrected reprint of the second (1989) edition.

[4] Benzel, S., Ge, Z., And Scovel, C. Elementary construction of higherorder Lie-Poisson integrators. Phys. Lett. A 174, 3 (1993), 229-232. 
[5] Cardin, F. On the geometrical Cauchy problem for the Hamilton-Jacobi equation. Nuovo Cimento B (11) 104, 5 (1989), 525-544.

[6] Cariñena, J. F., Gràcia, X., Marmo, G., Martínez, E., MuñozLecanda, M. G., and Román-Roy, N. Geometric Hamilton-Jacobi theory for nonholonomic dynamical systems. Int. J. Geom. Methods Mod. Phys. 7, 3 (2010), 431-454.

[7] Channell, P. J., And Scovel, C. Symplectic integration of Hamiltonian systems. Nonlinearity 3, 2 (1990), 231-259.

[8] Channell, P. J., And Scovel, J. C. Integrators for Lie-Poisson dynamical systems. Phys. D 50, 1 (1991), 80-88.

[9] Chaperon, M. On generating families. In The Floer memorial volume, vol. 133 of Progr. Math. Birkhäuser, Basel, 1995, pp. 283-296.

[10] de León, M., Marrero, J. C., and Martín de Diego, D. Linear almost Poisson structures and Hamilton-Jacobi equation. Applications to nonholonomic mechanics. J. Geom. Mech. 2, 2 (2010), 159-198.

[11] de León, M., Martín de Diego, D., And Vaquero, M. A HamiltonJacobi theory on Poisson manifolds. J. Geom. Mech. 6, 1 (2014), 121-140.

[12] Feng, K., And Qin, M. Symplectic geometric algorithms for Hamiltonian systems. Zhejiang Science and Technology Publishing House, Hangzhou; Springer, Heidelberg, 2010. Translated and revised from the Chinese original, With a foreword by Feng Duan.

[13] Fernandes, R. L., Ortega, J.-P., and Ratiu, T. S. The momentum map in Poisson geometry. Amer. J. Math. 131, 5 (2009), 1261-1310.

[14] Ferraro, S., de León, M., Marrero, J. C., Martín de Diego, D., And Vaquero, M. On the geometry of the Hamilton-Jacobi equation. Preprint, 2015.

[15] García-Toraño Andrés, E., Guzmán, E., Marrero, J. C., and Mestdag, T. Reduced dynamics and Lagrangian submanifolds of symplectic manifolds. J. Phys. A 47, 22 (2014), 225203, 24.

[16] Ge, Z. Generating functions, Hamilton-Jacobi equations and symplectic groupoids on Poisson manifolds. Indiana Univ. Math. J. 39, 3 (1990), 859-876.

[17] GE, Z. Equivariant symplectic difference schemes and generating functions. Phys. D 49, 3 (1991), 376-386.

[18] Ge, Z., And Marsden, J. E. Lie-Poisson Hamilton-Jacobi theory and Lie-Poisson integrators. Phys. Lett. A 133, 3 (1988), 134-139. 
[19] Goldstein, H. Classical mechanics, second ed. Addison-Wesley Publishing Co., Reading, Mass., 1980. Addison-Wesley Series in Physics.

[20] Hairer, E., Lubich, C., And Wanner, G. Geometric numerical integration, vol. 31 of Springer Series in Computational Mathematics. Springer, Heidelberg, 2010. Structure-preserving algorithms for ordinary differential equations, Reprint of the second (2006) edition.

[21] Holm, D. D. Geometric mechanics. Part I, second ed. Imperial College Press, London, 2011. Dynamics and symmetry.

[22] Iglesias-Ponte, D., De León, M., And Martín de Diego, D. Towards a Hamilton-Jacobi theory for nonholonomic mechanical systems. J. Phys. A 41, 1 (2008), 015205, 14.

[23] Kostant, B. Quantization and unitary representations. I. Prequantization. In Lectures in modern analysis and applications, III. Springer, Berlin, 1970, pp. 87-208. Lecture Notes in Math., Vol. 170.

[24] Marsden, J., And Weinstein, A. Reduction of symplectic manifolds with symmetry. Rep. Mathematical Phys. 5, 1 (1974), 121-130.

[25] Marsden, J. E., Misiołek, G., Ortega, J.-P., Perlmutter, M., AND RAtiu, T. S. Hamiltonian reduction by stages, vol. 1913 of Lecture Notes in Mathematics. Springer, Berlin, 2007.

[26] McLachlan, R. I., And Scovel, C. Equivariant constrained symplectic integration. J. Nonlinear Sci. 5, 3 (1995), 233-256.

[27] McLachlan, R. I., AND Scovel, C. A survey of open problems in symplectic integration. In Integration algorithms and classical mechanics (Toronto, ON, 1993), vol. 10 of Fields Inst. Commun. Amer. Math. Soc., Providence, RI, 1996, pp. 151-180.

[28] Meyer, K. R. Generic bifurcation of periodic points. Trans. Amer. Math. Soc. 149 (1970), 95-107.

[29] Meyer, K. R. Equivariant generating functions and periodic points. In New trends for Hamiltonian systems and celestial mechanics (Cocoyoc, 1994), vol. 8 of Adv. Ser. Nonlinear Dynam. World Sci. Publ., River Edge, NJ, 1996, pp. 289-299.

[30] Scovel, C., And Weinstein, A. Finite-dimensional Lie-Poisson approximations to Vlasov-Poisson equations. Comm. Pure Appl. Math. 47, 5 (1994), 683-709.

[31] Souriau, J.-M. Structure of dynamical systems, vol. 149 of Progress in Mathematics. Birkhäuser Boston, Inc., Boston, MA, 1997. A symplectic view of physics, Translated from the French by C. H. Cushman-de Vries, Translation edited and with a preface by R. H. Cushman and G. M. Tuynman. 
[32] Wang, H. Symmetric reduction and Hamilton-Jacobi equation of rigid spacecraft with a rotor. J. Geom. Symmetry Phys. 32 (2013), 87-111.

[33] Weinstein, A. Lectures on symplectic manifolds. American Mathematical Society, Providence, R.I., 1977. Expository lectures from the CBMS Regional Conference held at the University of North Carolina, March 8-12, 1976, Regional Conference Series in Mathematics, No. 29. 


\title{
On the Geometry of the Hamilton-Jacobi Equation and Generating Functions
}

\author{
Sebastián Ferraro ${ }^{* 1}$, Manuel de León ${ }^{\dagger 2}$, Juan Carlos Marrero ${ }^{\ddagger}$, \\ David Martín de Diego ${ }^{\S 2}$, and Miguel Vaquero $\mathbb{I}^{2}$ \\ ${ }^{1}$ Departamento de Matemática, Universidad Nacional del Sur \\ 8000 Bahía Blanca, Argentina \\ ${ }^{2}$ Instituto de Ciencias Matemáticas, ICMAT \\ c) Nicolás Cabrera, no 13-15, Campus Cantoblanco,UAM \\ 28049 Madrid, Spain \\ ${ }^{3}$ Departamento de Matemática Fundamental, ULL \\ c) Astrofísico Francisco Sánchez, s/n \\ 38206 La Laguna - Tenerife, Canary Islands, Spain
}

\begin{abstract}
In this paper we develop a geometric version of the Hamilton-Jacobi equation in the Poisson setting. Specifically, we "geometrize" what is usually called a complete solution of the Hamilton-Jacobi equation. We use some well-known results about symplectic groupoids, in particular cotangent groupoids, as a keystone for the construction of our framework. Our methodology follows the ambitious program proposed by A. Weinstein, [33], in order to develop geometric formulations of the dynamical behaviour of Lagrangian and Hamiltonian systems on Lie algebroids and Lie groupoids. This procedure allows us to take symmetries into account, and as a by-product we recover results from [5, 13, 15]. A theory of generating functions for the Poisson structures considered here is also developed following the same pattern. As an application we develop numerical methods based on generating functions, solving a longstanding problem of the area: how to obtain a generating function for the identity in Poisson manifolds. Some conclusions, current and future directions of research are shown at the end of the paper.
\end{abstract}

\footnotetext{
*sferraro@uns.edu.ar

†mdeleon@icmat.es

¥jcmarrer@ull.es

\$david.martin@icmat.es

I miguel.vaquero@icmat.es
} 
Keywords: Hamilton-Jacobi theory, symplectic groupoids, lagrangian submanifolds, symmetries, Poisson manifolds, Poisson integrators, generating function.

\section{Contents}

1 Introduction 3

1.1 Motivation ....................... 3

1.1.1 The Classical Case . . . . . . . . . . . . . . . 4

1.1.2 The Lie-Poisson Case . . . . . . . . . . . . . . . . 7

1.2 Summary . . . . . . . . . . . . . . . . . . 11

2 Symplectic Groupoids $\quad 12$

2.1 Definition of Symplectic Groupoids . . . . . . . . . . . . . . 12

2.2 Cotangent Groupoids . . . . . . . . . . . . . . . . . . 12

2.3 Example: Cotangent Bundle of the Gauge Groupoid . . . . . . . 14

2.4 Properties . . . . . . . . . . . . . . . . . . 15

3 Hamilton-Jacobi: The Lie Groupoid setting 16

3.1 The Geometric Setting _. . . . . . . . . . . . . . . 17

3.2 Main Result . . . . . . . . . . . . . . . . . . . . . . . . . 18

4 Generating functions for Poisson Manifolds 22

4.1 Motivation . . . . . . . . . . . . . . . . . . 22

4.2 Fundamental Lemma . . . . . . . . . . . . . . . . . . 23

4.3 Main Result . . . . . . . . . . . . . . . . . . . . . . . 24

4.4 Generating the identity: non-free canonical transformations . . . 24

4.4 .1 Motivation: Classical Case . . . . . . . . . . . . . . . 24

4.4 .2 Lie Algebras . . . . . . . . . . . . . . . . . . 26

4.4 .3 Action Lie Algebroids . . . . . . . . . . . . . . . 28

4.4 .4 Atiyah Algebroids . . . . . . . . . . . . . . . . . 29

5 Local Existence of Solution $\quad 29$

5.1 Existence of type I solutions for Lagrangian systems . . . . . . . 29

5.2 Method of Characteristics . . . . . . . . . . . . . 31

5.2 .1 Local Solution . . . . . . . . . . . . . . . . . 32

6 Examples 32

6.1 General Procedure . . . . . . . . . . . . . . . . . . . . 32

6.2 Rigid Body . . . . . . . . . . . . . . . . . . . . . 33

6.3 Heavy Top . . . . . . . . . . . . . . . . . . . . . 35

$\begin{array}{lll}7 & \text { Conclusions and Remarks } & 37\end{array}$ 
$\begin{array}{ll}\text { Appendix A Lie Algebroids } & 41\end{array}$

A.1 Definition . . . . . . . . . . . . . . . . . 42

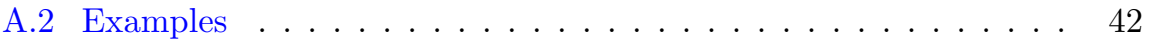

A.2.1 Vector Fields . . . . . . . . . . . . . . . . 42

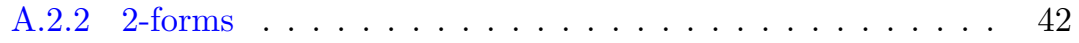

A.2.3 Poisson Manifolds . . . . . . . . . . . . . . . . . 42

A.3 The Poisson Structure of the Dual of a Lie Algebroid . . . . . . . 43

Appendix B Lie Groupoids $\quad 43$

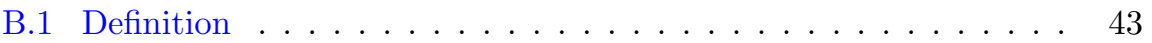

B.2 Lie Algebroid Associated to a Lie Groupoid . . . . . . . . . . . . . . . . 45

B.3 Examples of Lie groupoids . . . . . . . . . . . . . . . . . . . . . 46

B.3.1 Lie Groups . . . . . . . . . . . . . . . . . . . . 46

B.3.2 The Pair or Banal Groupoid . . . . . . . . . . . . . 47

B.3.3 Atiyah or Gauge Groupoids . . . . . . . . . . . . . . 47

B.3.4 Action Lie groupoids . . . . . . . . . . . . . . . 48

\section{Introduction}

\subsection{Motivation}

For the integration of the classical Hamilton's equations it is very useful to find canonical changes of coordinates that reduce the Hamiltonian function to a form such that the equations can be easily integrated. As a consequence, this shows that the initial equations are integrable. But, of course, the main problem is to find these particular canonical transformations. This problem is equivalent to the determination of a large enough number of solutions of the Hamilton-Jacobi equation. This is the objective of the representation of canonical transformations in terms of generating functions and leads to complete solutions of the Hamilton-Jacobi equations. The usefulness of this method is highlighted in the following quote by V.I. Arnold, (see [2], p. 233):

"The technique of generating functions for canonical transformations, developed by Hamilton and Jacobi, is the most powerful method available for integrating the differential equations of dynamics."

- V.I. Arnold

The procedure described above is well-known in the classical case, which geometrically corresponds to the cotangent bundle of the configuration manifold under consideration (or locally to any symplectic manifold using Darboux coordinates). Some research has been done in the Lie-Poisson case [15] as well. The goal of the following exposition is to introduce these two cases in order to motivate our future constructions, which deepen and generalize these results in a highly non-trivial way. 


\subsubsection{The Classical Case}

Let $Q$ be the $n$-dimensional configuration manifold of a mechanical system and let $\left(T^{*} Q, \omega_{Q}, H\right)$ be a Hamiltonian system. In this system $\omega_{Q}$ is the canonical symplectic structure of the cotangent bundle, $T^{*} Q$. Along this paper $\pi_{Q}$ : $T^{*} Q \rightarrow Q$ will be the natural projection of the cotangent bundle onto $Q$, and $H$ : $T^{*} Q \rightarrow \mathbb{R}$ will denote the Hamiltonian function. Associated to such Hamiltonian there is a Hamiltonian vector field, $X_{H}$ defined by $i_{X_{H}} \omega_{Q}=d H$. In natural cotangent coordinates $\left(q^{i}, p_{i}\right)$ the symplectic structure reads $\omega_{Q}=d q^{i} \wedge d p_{i}$ and the Hamiltonian vector field becomes

$$
X_{H}\left(q^{i}, p_{i}\right)=\frac{\partial H}{\partial p_{i}}\left(q^{i}, p_{i}\right) \frac{\partial}{\partial q^{i}}-\frac{\partial H}{\partial q^{i}}\left(q^{i}, p_{i}\right) \frac{\partial}{\partial p_{i}},
$$

so the equations of motion read

$$
\begin{aligned}
& \frac{d q^{i}}{d t}(t)=\frac{\partial H}{\partial p_{i}}\left(q^{i}(t), p_{i}(t)\right), \\
& \frac{d p_{i}}{d t}(t)=-\frac{\partial H}{\partial q^{i}}\left(q^{i}(t), p_{i}(t)\right)
\end{aligned}
$$

for $i=1, \ldots, n$.

For the sake of simplicity, and in order to clarify the main ideas of the paper, we start with a local coordinate description. Those ideas hold locally for any symplectic manifold in Darboux coordinates. The reader interested in the details and proofs of the results presented here is referred to $[1,2,16]$. Assume that we have found a function $S$ that depends on the time, $t$, the $\left(q^{i}\right)$-coordinates and $n$ parameters, say $\left(x^{i}\right), 1 \leq i \leq n$, so $S=S\left(t, q^{i}, x^{i}\right)$. If the function $S$ satisfies the following two conditions:

1. Hamilton-Jacobi equation:

$$
\frac{\partial S}{\partial t}\left(t, q^{i}, x^{i}\right)+H\left(q^{i}, \frac{\partial S}{\partial q^{i}}\left(t, q^{i}, x^{i}\right)\right)=K\left(t, x^{i}\right)
$$

where $K$ is a function that only depends on $t$ and $x^{i}$;

2. Non-degeneracy condition: $\operatorname{det}\left(\frac{\partial^{2} S}{\partial q^{i} \partial x^{j}}\right) \neq 0$,

then, by the implicit function theorem we can make the following change of coordinates $\left(t, q^{i}, p_{i}\right) \rightarrow\left(t, x^{i}, y_{i}\right)$ defined implicitly by

$$
\frac{\partial S}{\partial q^{i}}\left(t, q^{i}, x^{i}\right)=p_{i}, \quad-\frac{\partial S}{\partial x^{i}}\left(t, q^{i}, x^{i}\right)=y_{i}
$$

After some brief computations, one can see that in the new coordinates $\left(t, x^{i}, y_{i}\right)$ the equations of motion are again in Hamiltonian form, but now the Hamiltonian 
is the function $K\left(t, x^{i}\right)$, i.e. the equations (1) read now

$$
\begin{aligned}
& \frac{d x^{i}}{d t}(t)=\frac{\partial K}{\partial y_{i}}\left(x^{i}(t)\right)=0, \\
& \frac{d y_{i}}{d t}(t)=-\frac{\partial K}{\partial x^{i}}\left(x^{i}(t)\right)
\end{aligned}
$$

for $i=1, \ldots, n$.

Since $K$ only depends on the time and the $x^{i}$-coordinates, these equations are trivially integrable. Given an initial condition $\left(x_{0}^{i}, y_{i}^{0}\right)$ at time $t_{0}$, the curve

$$
t \rightarrow\left(x_{0}^{i}, y_{i}^{0}-\int_{t_{0}}^{t} \frac{\partial K}{\partial x^{i}}\left(t_{0}, x_{0}^{i}\right) d t\right)
$$

is the solution of equations (4) with initial condition $\left(x_{0}^{i}, y_{i}^{0}\right)$.

Remark 1. The equation

$$
\frac{\partial S}{\partial t}\left(t, q^{i}, x^{i}\right)+H\left(q^{i}, \frac{\partial S}{\partial q^{i}}\left(t, q^{i}, x^{i}\right)\right)=0
$$

appears frequently in the literature. If one is able to find the function $S$ satisfying equation (5) and the above non-degeneracy condition, that means that $K=0$ and so the equations of motion become $\frac{d x^{i}}{d t}(t)=0$ and $\frac{d y_{i}}{d t}(t)=0$. This means that, in the new coordinates, the system is in "equilibrium"; it does not evolve at all! The inverse of that change of variables gives the flow, up to an initial transformation given by $S\left(0, q^{i}, x^{i}\right)$, later on we will be clarify this claim. In general, any $S$ satisfying the non-degeneracy condition will induce a canonical transformation implicitly by the rule described above, which implies that Hamilton's equations in the $\left(q^{i}, p_{i}\right)$ coordinates will remain as Hamilton's equations in the $\left(x^{i}, y_{i}\right)$ for a new Hamiltonian, say $K$, which is related to the Hamilton-Jacobi equation by the expression

$$
\frac{\partial S}{\partial t}+H\left(t, q^{i}, \frac{\partial S}{\partial q^{i}}\right)=K\left(t, x^{i}, y_{i}\right) .
$$

Observe that equations (2) and (5) are particular instances of the last equation. We will elaborate on these and related issues in Section 4.

We proceed now to give a geometric framework for the previous procedure. The function $S$, satisfying (2), is interpreted here as a function on the product manifold $\mathbb{R} \times Q \times Q$ and so $\operatorname{Im}(d S)$ is a Lagrangian submanifold in $T^{*}(\mathbb{R} \times Q \times Q)$. Notice that we are thinking about the $\left(q^{i}\right)$ as coordinates on the first $Q$, and $\left(x^{i}\right)$ as coordinates on the second factor $Q$. This interpretation is directly related to the fact that we are describing here type $\boldsymbol{I}$ generating functions in the language of [16]. Other types of generating functions will be introduced along the next sections. On the other hand, consider the projections $\pi_{I}: T^{*}(\mathbb{R} \times Q \times Q) \rightarrow \mathbb{R} \times T^{*} Q, I=1,2$, defined by $\pi_{2}\left(t, e, x^{i}, y_{i}, q^{i}, p_{i}\right)=\left(t, q^{i}, p_{i}\right)$ and $\pi_{1}\left(t, e, x^{i}, y_{i}, q^{i}, p_{i}\right)=\left(t, e, x^{i},-y_{i}\right)$. 
With these geometric tools the non-degeneracy condition is equivalent to saying that $\pi_{I \mid \operatorname{Im}(d S)}$ are local diffeomorphisms for $I=1,2$. We assume here for simplicity that they are global diffeomorphisms, so we can consider the mapping $\pi_{1 \mid \operatorname{Im}(d S)} \circ\left(\pi_{2 \mid \operatorname{Im}(d S)}\right)^{-1}: \mathbb{R} \times T^{*} Q \rightarrow \mathbb{R} \times T^{*} Q$. The local argument follows with the obvious restrictions to open sets. This mapping can be easily checked to be the global description of the change of variables introduced in (3). The Hamilton-Jacobi equation (2) can be understood as the fact that $d S^{*}\left(\pi_{2}^{*} H+e\right)$ must be equal to $p r_{2}^{*} K$ for some $K \in C^{\infty}(\mathbb{R} \times Q)$, where $p r_{I}: \mathbb{R} \times Q \times Q \rightarrow \mathbb{R} \times Q, I=1,2$ are $p r_{1}\left(t, q^{i}, x^{i}\right)=\left(t, q^{i}\right), p r_{2}\left(t, q^{i}, x^{i}\right)=\left(t, x^{i}\right)$. The diagram below illustrates the situation.

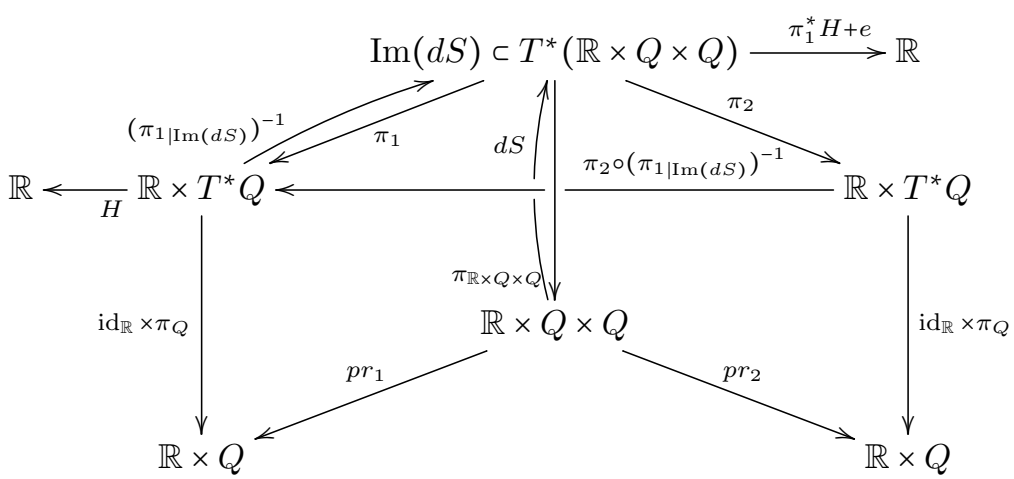

Figure 1: Geometric interpretation.

The transformation $\pi_{1 \mid \operatorname{Im}(d S)} \circ\left(\pi_{2 \mid \operatorname{Im}(d S)}\right)^{-1}$ satisfies

$$
\left(\pi_{1 \mid \operatorname{Im}(d S)} \circ\left(\pi_{2 \mid \operatorname{Im}(d S)}\right)^{-1}\right)_{*}\left(\frac{\partial}{\partial t}+X_{H}\right)=\frac{\partial}{\partial t}+X_{K},
$$

which is the geometric description of the transformation of equations (1) into (4).

Remark 2. We want to call the attention of the reader familiar with Lie groupoids or discrete mechanics about the geometric structure needed to handle this theory. From the above diagram if one removes the $\mathbb{R}$ factor, what we have is just a pair groupoid $Q \times Q$ and the corresponding cotangent groupoid $T^{*}(Q \times Q)$ with base the dual of its Lie algebroid $T^{*} Q$. We will show that the multiplication by the $\mathbb{R}$ factor conserves the groupoid and cotangent groupoid structures and for $\mathbb{R} \times Q \times Q$ the target and the source are exactly $p r_{1}$ and $p r_{2}$; furthermore, for the cotangent bundle $T^{*}(\mathbb{R} \times Q \times Q)$ the source and the target are just $\pi_{2}$ and $\pi_{1}$, introduced above.

Remark 3. Assuming that an initial condition is imposed at time 0 on $S$ such that the induced transformation is the identity $\operatorname{id}_{T^{*} Q}: T^{*} Q \rightarrow T^{*} Q$ at time 0 , then the inverse of the transformation introduced above, which happens to be $\pi_{1 \mid \operatorname{Im}(d S)} \circ\left(\pi_{2 \mid \operatorname{Im}(d S)}\right)^{-1}$, gives the flow of the Hamiltonian vector field $X_{H}$. 
Remark 4. The inverse of the transformation induced above, up to an initial condition on $S$ at time $t=0$ gives the flow of the Hamiltonian vector field $X_{H}$. Remark 5. We used a time-independent Hamiltonian, but actually the theory is exactly the same for time-dependent systems.

\subsubsection{The Lie-Poisson Case}

In this section we write in a geometric way the results about Hamilton-Jacobi theory for Lie-Poisson system, $\left(\mathfrak{g}^{*}, \Lambda, H\right)$, where

1. $\mathfrak{g}^{*}$ is the dual of the Lie algebra $\mathfrak{g}$ of a Lie group $G$;

2. $\Lambda$ is the canonical (-) Poisson structure on $\mathfrak{g}^{*}$, given by

$$
\{f, g\}(\mu)=-\mu([d f, d g])=\Lambda(d f, d g)
$$

where $f, g: \mathfrak{g}^{*} \rightarrow \mathbb{R}$.

3. $H: \mathfrak{g}^{*} \rightarrow \mathbb{R}$ is a Hamiltonian function.

These systems produce a dynamical system through the equation

$$
\dot{x}=X_{H}(x)=\Lambda^{\sharp}(d H)(x),
$$

where $d f\left(\Lambda^{\sharp}(H)\right)=\Lambda(d f, d H)$, and $X_{H}$ is called the Hamiltonian vector field. Detailed information about Lie-Poisson systems can be found, for instance, in [26]. Similar results to the ones that we are going to introduce now appeared for the first time in [15] and related information can also be found in [24]. In order to introduce that theory we need to define some mappings. The left and right momentum are the mappings $J_{L}: T^{*} G \rightarrow \mathfrak{g}^{*}$ and $J_{R}: T^{*} G \rightarrow \mathfrak{g}^{*}$ defined by $\left\langle J_{R}\left(\alpha_{g}\right), \xi\right\rangle=\left\langle\alpha_{g}, T_{e} L_{g}(\xi)\right\rangle$ and $\left\langle J_{L}\left(\alpha_{g}\right), \xi\right\rangle=\left\langle\alpha_{g}, T_{e} R_{g}(\xi)\right\rangle$. Let $S: \mathbb{R} \times G \rightarrow \mathbb{R}$ be a function such that the following conditions hold:

1. Hamilton-Jacobi equation: $\frac{\partial S}{\partial t}(t, g)+H\left(J_{R} \circ d S_{t}\right)=k(t)$, where $S_{t}$ is defined by $S_{t}(g)=S(t, g)$.

2. Non-degeneracy condition: let $\xi_{a}$ be a basis of $\mathfrak{g}$. Then we assume that $\overleftarrow{\xi}_{a}\left(\vec{\xi}_{b}\left(S_{t}\right)\right)$ is a regular matrix. Here $\overleftarrow{\xi}_{a}$ and $\vec{\xi}_{b}$ are the associated left-invariant and right-invariant vector fields respectively.

With the function $S$ at hand, we can define a transformation, analogous to (3). To make the exposition easier, we introduce the following diagram analogous to diagram in Figure 1: 


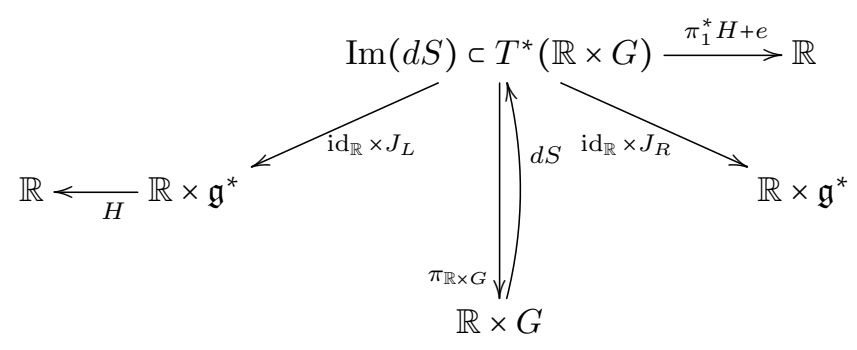

Figure 2: Lie-Poisson Setting.

where $\operatorname{id}_{\mathbb{R}} \times J_{L}\left(t, e, \alpha_{g}\right)=\left(t, J_{L}\left(\alpha_{g}\right)\right)$ and, in the same manner, we define $\operatorname{id}_{\mathbb{R}}$ $\times J_{R}$. The Hamilton-Jacobi equation is equivalent to saying that $d S^{*}\left(\left(\operatorname{id}_{\mathbb{R}} \times J_{L}\right)^{*}(H+\right.$ $e)$ ) is equal to a time function $k(t)$. It can also be checked that the nondegeneracy condition implies that $\operatorname{id}_{\mathbb{R}} \times J_{L}$ restricted to $\operatorname{Im}(d S)$ is a local diffeomorphism (it turns out that this is equivalent to $\operatorname{id}_{\mathbb{R}} \times J_{R}$ being a local diffeomorphism). Now we can define the Poisson mapping $\left(\operatorname{id}_{\mathbb{R}} \times J_{L}\right) \circ\left(\operatorname{id}_{\mathbb{R}} \times J_{R \mid \operatorname{Im}(d S)}\right)^{-1}$ : $\mathbb{R} \times \mathfrak{g}^{*} \rightarrow \mathbb{R} \times \mathfrak{g}^{*}$, henceforth denoted by $\hat{S}$. In this way we complete diagram in Figure 2 as follows:

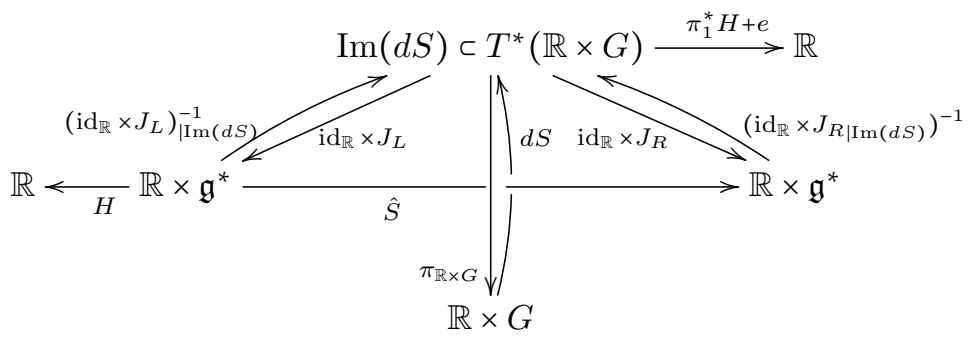

Figure 3: Geometric interpretation.

The main fact is, again, that $\hat{S}: \mathbb{R} \times \mathfrak{g}^{*} \rightarrow \mathbb{R} \times \mathfrak{g}^{*}$ verifies

$$
\hat{S}_{*}\left(\frac{\partial}{\partial t}+X_{H}\right)=\frac{\partial}{\partial t}
$$

which means that the Hamiltonian evolution is transformed into the trivial dynamics. With this method, we achieve a Poisson transformation which completely integrates the dynamics.

Remark 6. If we think about the underlying geometric structure, forgetting about the $\mathbb{R}$ factor, we have a Lie group, $G$, and its cotangent groupoid $T^{*} G$. The source and target of this cotangent groupoid are known to be the mappings $J_{L}$ and $J_{R}$. We will see that $T^{*}(\mathbb{R} \times G)$ is again a (symplectic) groupoid and that $\operatorname{id}_{\mathbb{R}} \times J_{L}$ and $\operatorname{id}_{\mathbb{R}} \times J_{R}$ are its source and target.

Remark 7. As in the previous case, the Hamiltonian can be time-dependent and the same results hold. 
With these two examples at hand it seems clear that the geometry of the theory can be described using cotangent Lie groupoids. The idea of using Lie groupoids to describe the Hamilton-Jacobi equation appeared for the first time in [13], as far as we know, and there it is pointed out that A. Weinstein was the first to notice that there may be a connection between generating functions and symplectic groupoids. As opposed to that general approach, we focus on giving a complete picture in the case of cotangent Lie groupoids. Cotangent Lie groupoids seem to be general enough to include all the interesting cases of mechanical systems, but at the same time they have interesting features that make them very useful in practical problems, which is our final goal. For instance, Darboux coordinates in the cotangent groupoid are always available, but finding them is not such an easy task in the case of a general symplectic groupoid. Furthermore, cotangent groupoids provide the natural framework to relate continuous and discrete Hamiltonian and Lagrangian dynamics, as was pointed by A. Weinstein in [33], following [30]. That viewpoint was exploited by some of the authors in [21, 22, 23]. A particular case of cotangent groupoid, the cotangent groupoid of an action Lie algebroid, was already suggested as the correct setting for a Hamilton-Jacobi theory in [32], although no more progress has been made in this direction so far. Moreover, in [32] the authors develop a finite-dimensional Poisson truncation, i.e. a finite-dimensional Poisson model, of the Poisson-Vlasov equation, an infinite-dimensional Poisson system. It turns out that the truncation happens to be the dual algebroid of an action groupoid, which is a particular case of the theory that we present here. The importance of the "oid theory" was already clear to the authors

"Our first derivation used the theory of Lie groupoids and Lie algebroids, and the Poisson structures on the duals of Lie algebroids. We were then able to eliminate the "oid" theory in favor of more well-known ideas on Poisson reduction. [...] The groupoid aspect of the theory also provides natural Poisson maps, useful in the application of Ruth type integration techniques, which do not seem easily derivable from the general theory of Poisson reduction".

-C. Scovel and A. Weinstein [32], p. 683.

Here we use that connection, symplectic groupoid-Poisson manifolds, to develop a general theory for Hamiltonian systems on the dual of an integrable Lie algebroid, a framework large enough to study all interesting Poisson Hamiltonian systems in classical mechanics. Using some well-known facts about symplectic groupoids, like the fact that the cotangent bundle of a Lie groupoid is a symplectic groupoid with base the dual of the associated Lie algebroid, then, a construction similar to the one outlined in the two examples above leads to the desired Hamiton-Jacobi theory. This theory allows us to seek for transformations which integrate Hamilton's equations in the same way we described above.

We also want to stress that besides the exact integration of the Hamilton's equations our motivation comes from the applications to numerical methods, 
which aims to develop, among other things, the Ruth type integrators pointed in the last quote, taken from [32]. Our examples go into this direction, although the analytical and dynamical applications should be exploited as well (see the last section). It is well-known that the theory of generating functions gives a family of symplectic numerical methods, [9, 17, 28]. There are numerous examples illustrating the superior preservation of phase-space structures and qualitative dynamics by symplectic integrators. These methods were extended to Lie groups in [15], but our approach is general enough to provide a general setting to develop new numerical methods on the dual bundle of Lie algebroids. We also stress the importance of this task, extending the symplectic integrators to the Poisson world, by a Peter Lax's quote that can be found in [9].

"In the late 1980s Feng Kang proposed and developed so-called symplectic algorithms for solving equations in Hamiltonian form. Combining theoretical analysis and computer experimentation, he showed that such methods, over long times, are much superior to standard methods. At the time of his death, he was at work on extensions of this idea to other structures."

$$
\text { - P. Lax }
$$

Our approach differs from the previous ones, [3, 5, 14, 15, 18, 29], in that we focus on the Lagrangian submanifolds instead of the generating functions themselves. We understand generating functions, as it has been done for many decades, as a device to describe Lagrangian submanifolds which are "horizontal" regarding a certain projection. That projection will only be defined locally in many cases, but that is enough for our applications. Let us clarify a little bit the situation. The most basic instance of this setting is the cotangent bundle $\left(T^{*} Q, \omega_{Q}\right)$ endowed with the canonical symplectic structure and the natural projection $\pi_{Q}: T^{*} Q \rightarrow Q$. Then we say that a Lagrangian submanifold, say $L$, is horizontal for $\pi_{Q}$ if $\left(\pi_{Q}\right)_{\mid L}: L \rightarrow Q$ is a diffeomorphism, equivalently, if there exists a 1 -form $\gamma$ such that $\operatorname{Im}(\gamma)=L$. This fact is a straightforward application of the implicit function theorem. By the Poincaré Lemma, at least locally, there exists $S$ defined on an open set of $Q$ such that $d S=\gamma$. In this sense, all the information of the Lagrangian submanifold $L$ can be encoded in a function, much easier to handle. Conditions on $L$ become PDEs in the unknown function $S$. This simple fact allowed us to come with analogues of the non-free canonical transformations, following the notation in [2]. They permit, in a very natural way, to generate the identity transformation as generating functions. This was an open problem, unsolved as far as we know:

"Is there a generating function for Lie-Poisson maps which generates the identity map via the coadjoint action of the identity group element?"

-R.I. McLachlan and C. Scovel. [29], p. 157.

This result is quite useful, as the classical type I generating functions are not 
enough even in the classical case. Furthermore, we give here results that show how Hamilton's equations change under a canonical transformation induced by a generating function, generalizing the equation (6) which is needed to develop Ruth type integrators. We also show local existence of solution and generalize the classical result that claims that the action functional $\left(\int L d t\right)$ is a solution of the Hamilton-Jacobi equation.

\subsection{Summary}

After the previous section, where we illustrated the motivation that led us to our results, we proceed in the following way.

Section 2 is devoted to the definition of symplectic groupoids. We include two appendices about Lie groupoids and Lie algebroids with all the definitions and properties, so the reader unfamiliar with them should find there the information needed to understand this paper. We summarize some of the most important properties of symplectic groupoids, because we are going to use them in Section 3 to develop our Hamilton-Jacobi theory. We provide several examples of symplectic groupoids and describe carefully the ones we are concerned with, cotangent groupoids.

In Section 3 we develop the main results of this paper. The main goal is to provide a Hamilton-Jacobi theory that allows us to look for transformations which integrate the Hamiltonian equations of motion, or at least, approximate them through Poisson automorphisms. This is done using the symplectic groupoid structure of the cotangent bundle of a groupoid, in particular, its dual pair structure.

Our Hamilton-Jacobi theory is used in Section 4 to produce a theory of generating functions for the Poisson systems we are concerned with. Although one can start the other way around, it seems that this way the presentation is more clear. We also summarize some of the results in [15] related to these generating functions.

In Section 5 we give a couple of results about local existence of solutions. Since we are dealing with a PDE, it is our duty to show existence at least. The first result generalizes the classical Jacobi's solution of the Hamilton-Jacobi equation given by the action. The second one is the method of characteristics, well-known in the literature.

Examples are given in Section 6. Of course, our theory recovers the classical situation via the pair groupoid and Ge-Marsden's framework via the Lie group case. Even in those cases, our approach clarifies the geometry and our results are stronger. We present there a general procedure to apply in order to develop numerical methods. Other examples given in the paper are the rigid body and the heavy-top. 


\section{Symplectic Groupoids}

Along this section we introduce what is going to be the geometric framework that allows us to "geometrize" the Hamilton-Jacobi equation in the next section. The basic definitions and properties are given, and several examples are provided. The usual definitions and notation about Lie groupoids and Lie algebroids are given in appendices $\mathrm{A}$ and $\mathrm{B}$. The basic references for this section are [6, 7]. We want to warn the reader, especially the one non experienced with groupoids, that in the end our constructions rely on the geometric structures of cotangent groupoids and not in the algebraic ones. That is, in order to follow our results it is enough to understand how to obtain the source and target of the cotangent groupoid and the results of Section 2.4. The main point is that cotangent groupoids provide a symplectic realization of the involved Poisson structures in a way that allows to control their Poisson automorphisms. To what extent the algebraic structures, multiplication and inversion, play a role here is still unknown to us.

\subsection{Definition of Symplectic Groupoids}

Let us now introduce the notion of symplectic groupoid. For the definitions of groupoids, Lie groupoids or Lie algebroids and the corresponding notation see the appendices.

Definition 1. A symplectic groupoid is a Lie groupoid $G \rightrightarrows M$ equipped with a symplectic form $\omega$ on $G$ such that the graph of the multiplication $m: G_{2} \rightarrow G$, that is, the set $\left\{(g, h, g h) \mid(g, h) \in G_{2}\right\}$, is a Lagrangian submanifold of $G \times G \times$ $(-G)$ with the product symplectic form, where $-G$ denotes $G$ endowed with the symplectic form $-\omega$.

Remark 8. It can be easily check that $(G, \omega)$ is a symplectic groupoid in the above sense if and only if the 2 -form $\omega$ is multiplicative. We say that the form $\omega$ is multiplicative iff

$$
m^{*} \omega=\pi_{1}^{*} \omega+\pi_{2}^{*} \omega
$$

where $\pi_{i}: G_{2} \rightarrow G, i=1,2$ are the projections over the first and second factor.

\subsection{Cotangent Groupoids}

This section constitutes a source of examples of symplectic groupoids. Since cotangent groupoids play an important role in our theory they deserve a whole subsection. Assume that $G \rightrightarrows M$ is a Lie groupoid with source and target $\alpha$ and $\beta$ respectively and identity section, inversion map and multiplication $\epsilon, \iota$ and $m$, then there is an induced Lie groupoid structure $T^{*} G \rightrightarrows A^{*} G$ which we define below (see Appendix B for notation). The cotangent bundle $T^{*} G$ turns out to be a symplectic groupoid with the canonical symplectic form $\omega_{G}$ (see $[6,7]$ ). Let us define the composition law on $T^{*} G$, which will be written as $\oplus_{T^{*} G}$, and let $\widetilde{\alpha}, \widetilde{\beta}, \widetilde{\epsilon}$ and $\widetilde{\iota}$ stand for the source, target, identity section and inversion map 
of the groupoid structure on $T^{*} G$ defined below. Each one of these maps will cover the corresponding structural map of $G$.

1. The source is defined in a way that the following diagram is commutative

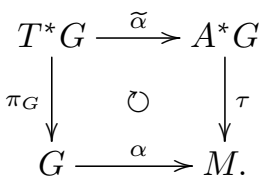

Assume that $g \in G_{y}^{x}=\alpha^{-1}(x) \cap \beta^{-1}(x)$ and let $X \in A_{x} G$. So, by the definition of Lie algebroid associated to a Lie groupoid, $X$ is a tangent vector at $\epsilon(x)$ that is tangent to $G^{x}=\alpha^{-1}(x)$. Then, since $\iota: G^{x} \rightarrow G_{x}$ (where $G_{x}=\beta^{-1}(x)$ ) is a diffeomorphism between the $\alpha$-fibers and $\beta$-fibers, we conclude that $-T_{\epsilon(x)}(X) \in T_{\epsilon(X)} G_{x}$, i.e., it is tangent to the $\beta$-fiber. Now, recalling that right multiplication is a bijection $R_{g}: G_{x} \rightarrow G_{y}$ we have that $T_{\epsilon(x)} R_{g}\left(-T_{\epsilon(x)} \iota(X)\right) \in T_{g} G$ and we can finally define

$$
\widetilde{\alpha}\left(\varpi_{g}\right)(X)=\varpi_{g}\left(-T_{\epsilon(x)}\left(R_{g} \circ \iota\right)(X)\right) .
$$

In particular, if we are dealing with a section $X \in \Gamma(\tau)$, the previous construction leads us to left-invariant vector fields:

$$
\widetilde{\alpha}\left(\varpi_{g}\right)(X)=\varpi_{g}(\vec{X}(g)), \text { for } X \in \Gamma(\tau),
$$

where $\tau: A G \rightarrow M$ is the Lie algebroid associated to $G$ (see equation (41) in Appendix B).

Notice that $\widetilde{\alpha}: T^{*} G \rightarrow A^{*} G$ is a surjective submersion.

2. In an analogous way, the target, $\widetilde{\beta}: T^{*} G \rightarrow A^{*} G$, is defined so that the following diagram commutes

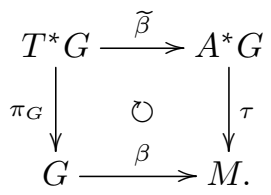

Now, given $\varpi_{g}$ and assuming that $g \in G_{y}^{x}$, since left multiplication is defined on the $\alpha$-fibers, $L_{g}: G^{y} \rightarrow G^{x}$, then $T_{\epsilon(x)} L_{g}(X) \in T_{g} G$ and we can define

$$
\widetilde{\beta}\left(\varpi_{g}\right)(X)=\varpi_{g}\left(T_{\epsilon(x)} L_{g}(X)\right) .
$$

In other words,

$$
\widetilde{\beta}\left(\varpi_{g}\right)(X)=\varpi_{g}(\overleftarrow{X}(g)), \text { for } X \in \Gamma(\tau)
$$

from equation (40), Appendix B. 
3. The identity map, $\widetilde{\epsilon}: A^{*} G \rightarrow T^{*} G$, is defined so that

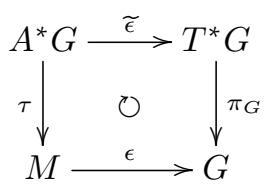

commutes. Take $v \in T_{\epsilon(x)} G$. We can obtain an element of $A_{x} G \subset T_{\epsilon(x)} G$ by computing $v-T(\epsilon \circ \alpha)(v)$. This is indeed tangent to an $\alpha$-fiber since $T \alpha$ of it is 0 , using an argument analogous to that in the definition of $\widetilde{\alpha}$. Then, for $\mu_{x} \in A_{x}^{*} G$, we define

$$
\widetilde{\epsilon}\left(\mu_{x}\right)(v)=\mu_{x}(v-T(\epsilon \circ \alpha)(v)) .
$$

4. The inversion map, $\widetilde{\imath}: T^{*} G \rightarrow T^{*} G$, is defined as a mapping from each $T_{g}^{*} G$ to $T_{g^{-1}}^{*} G$. If $X \in T_{g^{-1}} G$, then $T \iota(X) \in T_{g} G$, so we can define for $\varpi_{g} \in T_{g}^{*} G$

$$
\widetilde{\iota}\left(\varpi_{g}\right)(X)=-\varpi_{g}(T \iota(X)) .
$$

5. The groupoid operation $\oplus_{T^{*} G}$ is defined for these pairs $\left(\varpi_{g}, \nu_{h}\right) \in T^{*} G \times$ $T^{*} G$ satisfying the composability condition $\widetilde{\beta}\left(\varpi_{g}\right)=\widetilde{\alpha}\left(\nu_{h}\right)$, which in particular implies that $(g, h) \in G_{2}$ using diagrams (7) and (9). This condition can be rewritten as

$$
\varpi_{g} \circ T L_{g}=-\nu_{h} \circ T R_{h} \circ T \iota \quad \text { on } A_{\beta(g)} G \subset T_{\epsilon(\beta(g))} G .
$$

We define

$$
\left(\varpi_{g} \oplus_{T^{*} G} \nu_{h}\right)\left(T_{(g, h)} m\left(X_{g}, Y_{h}\right)\right)=\varpi_{g}\left(X_{g}\right)+\nu_{h}\left(Y_{h}\right),
$$

for $\left(X_{g}, Y_{h}\right) \in T_{(g, h)} G_{2}$. An explicit expression for $\oplus_{T^{*} G}$ using local bisections in the Lie groupoid $G$ can be found in [23].

Remark 9. Note from equation (8) (resp. (10)) that the definition of $\widetilde{\alpha}$ (resp. $\widetilde{\beta}$ ) is just given by "translation" via right-invariant vector fields (resp. leftinvariant).

Remark 10. Another interesting property is that the application $\pi_{G}: T^{*} G \rightarrow G$ is a Lie groupoid morphism over the vector bundle projection $\tau_{A^{*} G}: A^{*} G \rightarrow M$.

Remark 11. When the Lie groupoid $G$ is a Lie group, the Lie groupoid $T^{*} G$ is not in general a Lie group. The base $A^{*} G$ is identified with the dual of the Lie algebra $\mathfrak{g}^{*}$, and we have $\widetilde{\alpha}\left(\varpi_{g}\right)(\xi)=\varpi_{g}\left(T R_{g} \xi\right)$ and $\widetilde{\beta}\left(\varpi_{g}\right)(\xi)=\varpi_{g}\left(T L_{g} \xi\right)$, where $\varpi_{g} \in T_{g}^{*} G$ and $\xi \in \mathfrak{g}$.

\subsection{Example: Cotangent Bundle of the Gauge Groupoid}

We present now the cotangent groupoid of a gauge groupoid as an illustration of the previous constructions. The gauge groupoid is described in Section B.3.3. 
We assume here that the principal $G$-bundle $\pi: P \rightarrow M$ is trivial, so $P=G \times M$. Then, it is easy to see that $(P \times P) / G \equiv M \times M \times G$, with the identification $\left[\left(m^{1}, g^{1}, m^{2}, g^{2}\right)\right] \rightarrow\left(m^{1}, m^{2},\left(g^{1}\right)^{-1} g^{2}\right)$. Thus, $T^{*}((P \times P) / G) \equiv T^{*}(M \times M \times$ $G)$, and given $\left(\lambda_{m^{1}}, \mu_{m^{2}}, \nu_{g}\right) \in T^{*}(M \times M \times G)$, we have

1. The source, $\tilde{\alpha}$, is the map $\tilde{\alpha}\left(\lambda_{m^{1}}, \mu_{m^{2}}, \nu_{g}\right)=\left(-\lambda_{m^{1}}, J_{R}\left(\nu_{g}\right)\right)$.

2. The target, $\beta$, is the constant map $\tilde{\beta}\left(\lambda_{m^{1}}, \mu_{m^{2}}, \nu_{g^{1}}\right)=\left(\mu_{m^{2}}, J_{L}\left(\nu_{g}\right)\right)$.

3. The identity map is $\epsilon\left(\lambda_{m}, \nu\right)=\left(-\lambda_{m}, \lambda_{m}, \nu\right)$.

4. The inversion map is $\iota\left(\lambda_{m^{1}}, \mu_{m^{2}}, \nu_{g}\right)=\left(-\mu_{m^{2}}, \lambda_{m^{1}},-\nu_{g} \circ T_{g^{-1}}\right.$ inv $)$, where $i n v$ is the inversion in the Lie group $G$.

5. The multiplication is $m\left(\left(\lambda_{m^{1}}, \mu_{m^{2}}, \nu_{g}\right),\left(-\mu_{m^{2}}, \mu_{m^{3}}, \nu_{h}\right)\right)=\left(\lambda_{m^{1}}, \mu_{m^{3}}, \nu_{g} \circ\right.$ $\left.T_{g h} R_{h^{-1}}=\nu_{h} \circ T_{g h} L_{g^{-1}}\right)$.

Remark 12. Easier-to-handle expressions can be obtained by trivializing $T^{*} G \equiv$ $G \times \mathfrak{g}^{*}$ in the product $T^{*}(M \times M \times G) \equiv T^{*} M \times T^{*} M \times T^{*} G$.

\subsection{Properties}

The following theorem will be crucial in the next constructions (see $[6,7]$ for a proof, as well as the references therein):

Theorem 2. Let $G \rightrightarrows M$ be a symplectic groupoid, with symplectic 2-form $\omega$. We have the following properties:

1. For any point $g \in G_{y}^{x}$, the subspaces $T_{g} G_{y}$ and $T_{g} G^{x}$ of the symplectic vector space $\left(T_{g} G, \omega_{g}\right)$ are mutually symplectic orthogonal. That is,

$$
T_{g} G_{y}=\left(T_{g} G^{x}\right)^{\perp} .
$$

2. The submanifold $\epsilon(M)$ is a Lagrangian submanifold of the symplectic manifold $(G, \omega)$.

3. The inversion map $\iota: G \rightarrow G$ is an anti-symplectomorphism of $(G, \omega)$, that is, $\iota^{*} \omega=-\omega$.

4. There exists a unique Poisson structure $\Pi$ on $M$ for which $\beta: G \rightarrow M$ is a Poisson map, and $\alpha: G \rightarrow M$ is an anti-Poisson map (that is, $\alpha$ is a Poisson map when $M$ is equipped with the Poisson structure $-\Pi)$.

Remark 13. The theorem above states that the $\alpha$-fibers and $\beta$-fibers are symplectically orthogonal. A pair of fibrations satisfying that property are called a dual pair. This dual pair property will be the keystone of our construction.

Remark 14. When dealing with the symplectic groupoid $T^{*} G$, where $G$ is a groupoid, the Poisson structure on $A^{\star} G$ is the (linear) Poisson structure of the dual of a Lie algebroid (see Appendix A.3). 
The next theorem is the core of our Hamilton-Jacobi theory. It basically says that Lagrangian bisections of a symplectic groupoid induce Poisson transformations in the base.

Theorem 3 (see [6]). Let $G$ be a symplectic goupoid with source and target $\alpha$ and $\beta$ respectively. Let $L$ be a Lagrangian submanifold of $G$ such that $\alpha_{\mid L}$ is a (local) diffeomorphism. Then

1. $\beta_{\mid L}: L \rightarrow M$ is a (local) diffeomorphism as well.

2. The mapping $\hat{L}=\beta \circ\left(\alpha_{\mid L}\right)^{-1}: M \rightarrow M$ and its inverse, $\alpha \circ\left(\beta_{\mid L}\right)^{-1}$, are (local) Poisson isomorphisms.

Remark 15. We wrote in the above theorem the mappings in the global diffeomorphism case. Of course, when the diffeomorphism is local, these maps are restricted to the corresponding open sets.

\section{Hamilton-Jacobi: The Lie Groupoid setting}

In this section we develop our Hamilton-Jacobi theory using the geometric structures introduced along the previous sections. We encourage the reader, especially the reader not familiar with Lie groupoids and Lie algebroids, to keep in mind the two instanced of the Hamilton-Jacobi theory sketched in the introduction of this paper (the classical and the Lie-Poisson cases). Our construction mimics what happens in those cases.

Let us start with a (possibly time-dependent) Hamiltonian system on the dual of an integrable Lie algebroid $\left(\mathbb{R} \times A^{*} G, \Pi_{\mathbb{R}}, H\right)$. We clarify now the situation.

1. $\mathbb{R} \times A^{*} G$ is the extended-phase space, that is, the product of the phase space $A^{*} G$ and the time $\mathbb{R}$. We are assuming that our (possible timedependent) Hamiltonian system evolves on the dual of a Lie algebroid. This framework is enough to cover all interesting cases:

(a) cotangent bundles;

(b) duals of Lie algebras (Lie-Poisson systems);

(c) duals of action Lie algebroids (semi-direct products: heavy top, truncated Vlasov-Poisson...);

(d) reduced systems (dual of the Atiyah algebroid),

among others. Notice that this is the space where A. Weinstein points out that the dynamics of Hamiltonian systems should take place in [33].

2. $\Pi_{\mathbb{R}}$ is the Poisson structure in $\mathbb{R} \times A^{*} G$ given by the product of the 0 Poisson structure on $\mathbb{R}$ and the natural linear Poisson structure $\Pi$ on $A^{*} G$ described in Section A.3 of Appendix A. So $\Pi_{\mathbb{R}}=0 \times \Pi$. 
3. $H: \mathbb{R} \times A^{*} G \rightarrow \mathbb{R}$ is the Hamiltonian function. Once that function is given, the dynamics is produced by the suspension of the Hamiltonian vector field, i.e., $\frac{\partial}{\partial t}+X_{H}$, where $X_{H}=\Pi_{\mathbb{R}}^{\sharp}(d H)$, which is a time-dependent vector field in $\mathbb{R} \times A^{*} G$.

\subsection{The Geometric Setting}

Before going to our Hamilton-Jacobi theory, we need to introduce one more construction. It basically says that the $\mathbb{R}$ factor we mention in Remark 2 and Remark 6 does not modify the groupoid structure. This theorem was already sketched in [13].

Proposition 4. Let $G$ be a Lie groupoid with source, target, identity map, inversion and multiplication $\alpha, \beta, \epsilon, \iota$ and $m_{\mathbb{R}}$. Then the manifold $\mathbb{R} \times G$ is a Lie groupoid with base $\mathbb{R} \times M$ and with structure mappings

$$
\begin{array}{rlll}
\alpha_{\mathbb{R}}: & \mathbb{R} \times G & \longrightarrow & \mathbb{R} \times M \\
& (t, g) & \rightarrow & \alpha_{\mathbb{R}}(t, g)=(t, \alpha(g)) \\
\beta_{\mathbb{R}}: & \mathbb{R} \times G & \longrightarrow & \mathbb{R} \times M \\
& (t, g) & \rightarrow & \beta_{\mathbb{R}}(t, g)=(t, \beta(g)) \\
\epsilon_{\mathbb{R}}: & \mathbb{R} \times M & \longrightarrow & \mathbb{R} \times G \\
& (t, x) & \rightarrow & \epsilon_{\mathbb{R}}(t, x)=(t, \epsilon(g)) \\
& & & \\
\iota_{\mathbb{R}}: & \mathbb{R} \times G & & \mathbb{R} \times G \\
& (t, g) & \rightarrow & \iota_{\mathbb{R}}(t, g)=(t, \iota(g)) \\
m_{\mathbb{R}}: & (\mathbb{R} \times G)_{2} & \longrightarrow & \mathbb{R} \times G \\
& ((t, g),(t, h)) & \rightarrow & m_{\mathbb{R}}((t, g),(t, h))=(t, m(g, h)) .
\end{array}
$$

Notice that the condition $\beta_{\mathbb{R}}\left(t_{1}, g\right)=\alpha_{\mathbb{R}}\left(t_{2}, h\right)$ is equivalent to $t_{1}=t_{2}$ and $\beta(g)=$ $\alpha(h)$.

The proof is obvious and we omit it. If $A G$ is the Lie algebroid associated to $G$, then the Lie algebroid associated to $\mathbb{R} \times G$ is just $\tau_{\mathbb{R}}: \mathbb{R} \times A G \rightarrow \mathbb{R} \times M$, where $\tau_{\mathbb{R}}(t, X)=(t, \tau(X))$ and the addition is just $(t, X)+(t, Y)=(t, X+Y)$ for $t \in \mathbb{R}$ and $X, Y \in A G$. The anchor and the Lie bracket on sections have analogous expressions. Actually one can think on $\mathbb{R} \times G$ and $\mathbb{R} \times A G$ as "time-dependent" structures, although we think about them just as groupoids. The dual of this Lie algebroid is simply $A^{*}(\mathbb{R} \times G)=\mathbb{R} \times A^{*} G$. The cotangent bundle of $\mathbb{R} \times G$ is a (symplectic) Lie groupoid with base $\mathbb{R} \times A^{*} G$ and structure maps $\widetilde{\alpha_{\mathbb{R}}}, \widetilde{\beta_{\mathbb{R}}}, \widetilde{\epsilon_{\mathbb{R}}}, \widetilde{\iota_{\mathbb{R}}}$ and $\widetilde{m_{\mathbb{R}}}$. The next diagram summarizes the situation, note the commutativity: 


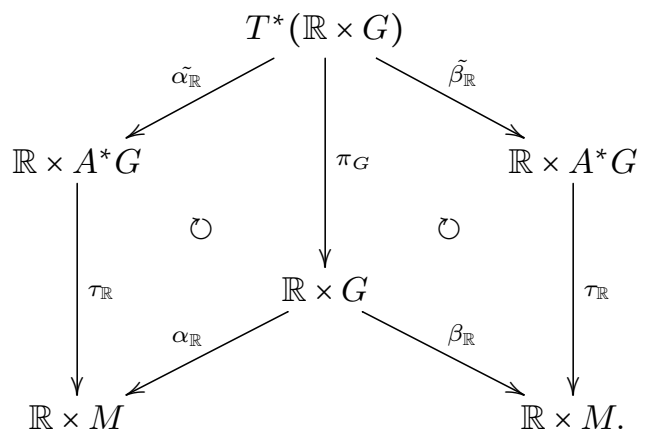

Figure 4: Geometric interpretation.

The reader should note that the diagram above is just the generalization of diagrams in Figures 1 and 2. When $G$ is the pair groupoid or a Lie group we recover these two settings. A point of $T^{*}(\mathbb{R} \times G)$ is given by $\left(t, e, \alpha_{g}\right)$, representing the cotangent vector $e d t+\alpha_{g}$, that is, $e$ is the time conjugate momentum. With this notation, the maps $\widetilde{\alpha_{\mathbb{R}}}$ and $\widetilde{\beta_{\mathbb{R}}}$ read as follows

$$
\begin{aligned}
& \widetilde{\alpha_{\mathbb{R}}}: \quad T^{*}(\mathbb{R} \times G) \quad \longrightarrow \mathbb{R} \times A^{*} G \\
& \left(t, e, \varpi_{g}\right) \quad \rightarrow \quad \widetilde{\alpha_{\mathbb{R}}}\left(t, e, \varpi_{g}\right)=\left(t, \widetilde{\alpha}\left(\varpi_{g}\right)\right) \\
& \widetilde{\beta_{\mathbb{R}}}: \quad T^{*}(\mathbb{R} \times G) \quad \longrightarrow \mathbb{R} \times A^{*} G \\
& \left(t, e, \varpi_{g}\right) \quad \rightarrow \quad \widetilde{\beta_{\mathbb{R}}}\left(t, e, \varpi_{g}\right)=\left(t, \widetilde{\beta}\left(\varpi_{g}\right)\right) .
\end{aligned}
$$

Remark 16. It is easy to see that the Poisson structure on $A^{*}(\mathbb{R} \times G)=\mathbb{R} \times A^{*} G$ is given by the product of the 0 Poisson structure on $\mathbb{R}$ and the natural Poisson structure on $A^{*} G$, say $\Pi$. So the Poisson structure on $\mathbb{R} \times A^{*} G$ is just the one described at the beginning of the section, recall that we refer to this structure as $\Pi_{\mathbb{R}}$. Recall that by Theorem 2 we have a dual pair structure, considering the natural symplectic structure $\omega_{\mathbb{R} \times G}$ on $T^{*}(\mathbb{R} \times G)$ and the aforementioned Poisson structure $\prod_{\mathbb{R}}$ on $\mathbb{R} \times A^{*} G$.

Now let us construct a function $H^{e}: T^{*}(\mathbb{R} \times G) \rightarrow \mathbb{R}$ out of the Hamiltonian $H$ that we called the extended Hamiltonian associated to $H$. Define $H^{e}\left(t, e, \varpi_{g}\right)=$ $\widetilde{\beta_{\mathbb{R}}}{ }^{*} H+e$. Finally, we state the Hamilton-Jacobi theorem.

\subsection{Main Result}

The next theorem is the generalization of the results shown in Section 1.1.

Theorem 5 (Hamilton-Jacobi). Let $S: \mathbb{R} \times G \rightarrow \mathbb{R}$ be a function and assume that the following conditions hold:

1. Hamilton-Jacobi equation, $H^{e} \circ d S=K$, where $K=\alpha_{\mathbb{R}}^{*} k$ for some function $k: \mathbb{R} \times M \rightarrow \mathbb{R}$. That means that $K$ is a $\alpha_{\mathbb{R}}$-basic function. 
2. Non-degeneracy condition, $\widetilde{\alpha_{\mathbb{R}}} \mid \operatorname{Im}(d S): \operatorname{Im}(d S) \rightarrow \mathbb{R} \times A^{*} G$ is a (local) diffeomorphism.

Then the induced Poisson isomorphism $\widetilde{\alpha_{\mathbb{R}}} \circ\left(\widetilde{\beta_{\mathbb{R}}} \mid \operatorname{Im}(d S)\right)^{-1}: \mathbb{R} \times A^{*} G \rightarrow \mathbb{R} \times A^{*} G$ (henceforth denoted $\hat{S}$ ) satisfies

$$
\hat{S}_{*}\left(\frac{\partial}{\partial t}+X_{H}\right)=\frac{\partial}{\partial t}+X_{\hat{k}}
$$

where $\hat{k}=\tau_{\mathbb{R}}^{*} k$.

Remark 17. 1. The Hamilton-Jacobi equation is easily seen to recover the two examples discussed in the introduction. In the classical case it reads as (2). In the general case it becomes

$$
\frac{\partial S}{\partial t}+H\left(t, \widetilde{\beta}\left(\frac{\partial S}{\partial g}\right)\right)=k(t, \alpha(g))
$$

where $d S=\frac{\partial S}{\partial t} d t+\frac{\partial S}{\partial g} d g$ comes from the identification $T^{*}(\mathbb{R} \times G)=T^{*} \mathbb{R} \times$ $T^{*} G$, so $\frac{\partial S}{\partial t} d t \in T^{*} \mathbb{R}$ and $\frac{\partial S}{\partial g} d g \in T^{*} G$. The reader should compare this expression with the Hamilton-Jacobi equations in the classical and Lie group cases.

2. The non-degeneracy condition is the geometric description of the nondegeneracy previously used $\left(\operatorname{det}\left(\frac{\partial^{2} S}{\partial q^{i} \partial Q^{j}}\right) \neq 0\right.$ and $\overleftarrow{\xi}_{a}\left(\vec{\xi}_{b}\left(S_{t}\right)\right)$ be regular $)$ It can be stated saying that $\operatorname{Im}(d S)$ is a bisection.

3. Obviously, this theorem will only be useful if the new Hamiltonian dynamics $\frac{\partial}{\partial t}+X_{\hat{k}}$ are trivially integrable, as before. Take coordinates adapted to the fibration, say $t, x^{i}, y_{j}, i=1, \ldots, n, j=1, \ldots, m$, where $t$ is the $\mathbb{R}$-coordinate, $n$ the dimension of $M$ and $m$ the dimension of the $\alpha$-fibers. Thus, the equations of motion read

$$
\begin{aligned}
& \frac{d x^{i}}{d t}=\left\{x^{i}, \hat{k}\left(t, x^{i}\right)\right\}=0 \\
& \frac{d y_{j}}{d t}=\left\{y_{j}, \hat{k}\left(t, x^{i}\right)\right\}=\rho_{j}^{k}\left(x^{i}\right) \frac{\partial \hat{k}}{\partial x^{k}}\left(t, x^{i}\right)
\end{aligned}
$$

where $i=1, \ldots, n, j=1, \ldots, m$. So if $\hat{k}$ is time-independent, given an initial condition $\left(x_{0}^{i}, y_{j}^{0}\right)$ at time $t_{0}$ the solution of Hamilton's equations is

$$
t \rightarrow\left(t, x_{i}^{0}, y_{j}^{0}+\rho_{j}^{k}\left(x_{i}^{0}\right) \frac{\partial \hat{k}}{\partial x^{k}}\left(x_{i}^{0}\right)\left(t-t_{0}\right)\right) .
$$

Otherwise, in the time-dependent setting the solution is just given by integration

$$
t \rightarrow\left(t, x_{i}^{0}, \int_{t_{0}}^{t} \rho_{j}^{k}\left(x_{0}^{i}\right) \frac{\partial \hat{k}}{\partial x_{0}^{k}}\left(s, x_{0}^{i}\right) d s+y_{i}^{0}\right) .
$$


See Appendix A, Section A.3 for a detailed account of this Poisson structure.

Proof: We will prove the global diffeomorphism case, i.e., when $\widetilde{\alpha_{\mathbb{R}}} \mid \operatorname{Im}(d S)$ : $\operatorname{Im}(d S) \rightarrow \mathbb{R} \times A^{*} G$ is a global diffeomorphism. In the local diffeomorphism case, the argument follows by restricting the mappings to the corresponding open sets. For the sake of clarity of the exposition, we will divide the proof into two cases. In the first case, we assume that the stronger equation $H^{e} \circ d S=E$ holds $(E \in \mathbb{R}$ is a constant). In the second step we prove the general case, i.e., when $H^{e} \circ d S=K$, where $K$ is a function.

First case: Assume that $S$ is such that $H^{e} \circ d S=E$. Let $X_{H^{e}}$ be the Hamiltonian vector field on $T^{*}(\mathbb{R} \times G)$ associated to $H^{e}$, that is, $i_{X_{H^{e}}}\left(\omega_{\mathbb{R} \times G}\right)=d H^{e}$. Then $d H^{e} \in T \operatorname{Im}(d S)^{\circ}$, where $W^{\circ}$ means "the annihilator of the subspace $W$ ". Since $\operatorname{Im}(d S)$ is a Lagrangian submanifold (i.e. $T \operatorname{Im}(d S)^{\perp}=T \operatorname{Im}(d S)$, where ${ }^{\perp}$ stands for the symplectic orthogonal), then $X_{H^{e}} \in T \operatorname{Im}(d S)$. So the Hamiltonian vector field $X_{H^{e}}$ is tangent to $\operatorname{Im}(d S)$. Let us now analyze $X_{H^{e}}$, since $H^{e}={\widetilde{\beta_{\mathbb{R}}}}^{*} H+e$, then $X_{H^{e}}=X_{{\widetilde{\beta_{\mathbb{R}}}}^{*} H}+X_{e}$. Since the symplectic structure on $T^{*}(\mathbb{R} \times G)$ is the sum $d t \wedge d e+\omega_{G}$ we conclude that $X_{e}=\frac{\partial}{\partial t}$. On the other hand, $\widetilde{\beta_{\mathbb{R}}}$ is Poisson map, so $\widetilde{\beta_{\mathbb{R} *}}\left(X_{\widetilde{\beta_{\mathbb{R}}}}{ }^{*}\right)=X_{H}{ }^{1}$, where $X_{H}$ is the vector field defined on $\mathbb{R} \times A^{*} G$ by $X_{H}=\Pi_{\mathbb{R}}^{\sharp}(d H)^{2}$. Summarizing, we deduce

$$
\widetilde{\beta_{\mathbb{R}_{*}}}\left(X_{H^{e}}\right)=\frac{\partial}{\partial t}+X_{H}
$$

which is the dynamics of the Hamiltonian system $\left(\mathbb{R} \times A^{*} G, \Pi_{\mathbb{R}}, H\right)$.

Nevertheless, since $\widetilde{\beta_{\mathbb{R}}}$ and $\widetilde{\alpha_{\mathbb{R}}}$ are a dual pair, i.e., the $\widetilde{\beta_{\mathbb{R}}}$-fibers and $\widetilde{\alpha_{\mathbb{R}}}$ fibers are symplectically orthogonal $\left(\operatorname{ker}\left(T \widetilde{\beta_{\mathbb{R}}}\right)^{\perp}=\operatorname{ker}\left(T \widetilde{\alpha_{\mathbb{R}}}\right)\right)$ and since $d \widetilde{\widetilde{\beta}_{\mathbb{R}}}{ }^{*} H \epsilon$ $\operatorname{ker}\left(T \widetilde{\beta_{\mathbb{R}}}\right)^{\circ}$ by definition, then $X_{\widetilde{\beta_{\mathbb{R}}} H}=\omega_{\mathbb{R} \times G}^{\sharp}\left(\widetilde{d}\left(\beta_{\mathbb{R}}^{*} H\right)\right) \in \operatorname{ker}\left(T \widetilde{\alpha_{\mathbb{R}}}\right)^{3}$. This last equation implies that $\widetilde{\alpha_{\mathbb{R}}}\left(X_{\widetilde{\beta_{\mathbb{R}}}{ }^{*}}\right)=0$ and thus

$$
\widetilde{\alpha_{\mathbb{R}} *}\left(X_{H^{e}}\right)=\frac{\partial}{\partial t} \text {. }
$$

Now we proceed to compute $\bar{S}_{*}\left(\frac{\partial}{\partial t}+X_{H}\right)$. By equation (16) and since $X_{H^{e}}$ is tangent to $\operatorname{Im}(d S)$, then $\left(\widetilde{\beta_{\mathbb{R}}} \mid \operatorname{Im}(d S)\right)_{*}\left(X_{H^{e}}\right)=\frac{\partial}{\partial t}+X_{H}$ or equivalently, reversing the arguments

$$
\left(\widetilde{\beta_{\mathbb{R}} \operatorname{Im}(d S)}\right)_{*}^{-1}\left(\frac{\partial}{\partial t}+X_{H}\right)=\left(X_{H^{e}}\right) \mid \operatorname{Im}(d S)
$$

Combining equation (18) and equation (17) we obtain in a straightforward way

$$
\hat{S}_{\star}\left(\frac{\partial}{\partial t}+X_{H}\right)=\frac{\partial}{\partial t}
$$

\footnotetext{
${ }^{1}$ This is a well-known fact in Poisson geometry. See, for instance, [26].

${ }^{2}$ We are aware that we are using the same notation for Hamiltonian vector fields on different manifolds, but since the corresponding Hamiltonian functions are defined in different manifolds, there is no room for confusion.

${ }^{3}$ Recall that by definition $\operatorname{ker}\left(T \widetilde{\beta_{\mathbb{R}}}\right)^{\perp}=\omega_{\mathbb{R} \times G}^{\sharp}\left(\operatorname{ker}\left(T \widetilde{\beta_{\mathbb{R}}}\right)^{\circ}\right)$.
} 
and we conclude the first step of the proof.

Remark 18. In this case, the inverse of the map above, $\widetilde{\beta_{\mathbb{R}}} \circ\left(\widetilde{\alpha_{\mathbb{R}}} \mid \operatorname{Im}(d S)\right)^{-1}$, is up to an initial condition the flow of the Hamiltonian vector field. This means that the transformation satisfies

$$
\frac{\partial \hat{S}(t, g)}{\partial t}=X_{H}(\hat{S}(t, g))
$$

but $\hat{S}(0, g)$ is not necessarily the identity.

Second case: Assume now that the equation $H^{e} \circ d S=K$ holds, where $K=$ $\alpha_{\mathbb{R}}^{*} k$ for some function $K: \mathbb{R} \times M \rightarrow \mathbb{R}$. We will denote by $\hat{k}$ the function $\tau_{\mathbb{R}}^{*} k$ and $\underline{K}={\widetilde{\alpha_{\mathbb{R}}}}^{*} \hat{k}$. Notice that we have $\underline{K}={\widetilde{\alpha_{\mathbb{R}}}}^{*} \hat{k}=\pi_{\mathbb{R} \times G}^{*} K$ by commutativity of diagram (4). Now, since $H^{e} \circ d S=K$ we can claim that $\left(H^{e}-\underline{K}\right) \circ d S=$ 0 . The same argument used in step one, ensures that $X_{\left(H^{e}-\underline{K}\right)}$ is tangent to $\operatorname{Im}(d S)$. Notice that this argument is just the standard procedure used in proof of the Hamilton-Jacobi theorem on cotangent bundles [1]. Of course, $X_{\left(H^{e}-\underline{K}\right)}=X_{H^{e}}-X_{\underline{K}}$, and recalling that $\widetilde{\beta_{\mathbb{R}}}$ and $\widetilde{\alpha_{\mathbb{R}}}$ are Poisson and anti-Poisson mappings and that $H^{e}=\widetilde{\beta_{\mathbb{R}}}{ }^{*} H+e$, and $\bar{k}=\widetilde{\alpha_{\mathbb{R}}}{ }^{*} \hat{k}$ then

$$
\begin{aligned}
& \widetilde{\beta_{\mathbb{R}_{*}}}\left(X_{H^{e}}\right)=\frac{\partial}{\partial t}+X_{h} \\
& \widetilde{\beta_{\mathbb{R}_{*}}}\left(-X_{\bar{K}}\right)=0 \\
& \widetilde{\alpha_{\mathbb{R} *}}\left(X_{H^{e}}\right)=\frac{\partial}{\partial t} \\
& \widetilde{\alpha_{\mathbb{R} *}}\left(-X_{\bar{K}}\right)=X_{\hat{k}}
\end{aligned}
$$

The equations (20) and (22) hold by the same argument used in the first step. The equations (21) and (23) hold using the dual pair structure and that $\underline{K}=$ $\widetilde{\alpha_{\mathbb{R}}} *(\hat{k})$. Now we can compute $\hat{S}_{*}\left(\frac{\partial}{\partial t}+X_{H}\right)$ following the same pattern we used in the first step.

First, notice that (by $(20)$ and $(21))$ since $\widetilde{\beta_{\mathbb{R}_{*}}}\left(X_{H^{e}}-X_{\underline{K}}\right)=\frac{\partial}{\partial t}+X_{H}$ and recalling that $X_{H^{e}}-X_{\underline{K}}$ is tangent to $\operatorname{Im}(d S)$ then

$$
\left(\widetilde{\beta_{\mathbb{R}} \mid \operatorname{Im} d S}\right)_{*}^{-1}\left(\frac{\partial}{\partial t}+X_{H}\right)=\left(X_{H^{e}}-X_{\underline{K}}\right)_{\mid \operatorname{Im}(d S)}
$$

On the other hand, adding equation (22) and equation (23), we can conclude $\hat{S}_{*}\left(\frac{\partial}{\partial t}+X_{H}\right)=\left(\widetilde{\alpha_{\mathbb{R}}}\right)_{*}\left(\left(\widetilde{\beta_{\mathbb{R}}} \mid \operatorname{Im} d S\right)_{*}^{-1}\left(\frac{\partial}{\partial t}+X_{H}\right)\right)=\left(\widetilde{\alpha_{\mathbb{R}}}\right)_{*}\left(X_{H^{e}}-X_{\underline{K}}\right)=\frac{\partial}{\partial t}+X_{\hat{k}}$. 
4 GENERATING FUNCTIONS FOR POISSON MANIFOLDS

Remark 19. Of course, one can substitute $d S$ by a closed 1 -form, say $\gamma$, and all the previous claims hold. Moreover, one can think about any Lagrangian bisection $L$, (that is exactly the non-degeneracy condition) and then the HamiltonJacobi equation becomes just $H_{\mid L}^{e}=\left(\pi_{\mathbb{R} \times G}^{*} K\right)_{\mid L}$. Notice that the same proof applies and therefore we can conclude that the induced transformation, say $\hat{L}: \mathbb{R} \times A^{*} G \rightarrow \mathbb{R} \times A^{*} G$, satisfies equation (14). This observation is very important, along the next section we will exploit this Lagrangian submanifold viewpoint to develop our theory of generating functions. The main application of that approach, that is, thinking about Lagrangian submanifolds which are not horizontal (i.e. $\operatorname{Im}(\gamma)$ for a closed 1-form $\gamma$ ), is to deal with generating functions which are not of the "first type". In the classical theory, it means that one can allow $S$ to be dependent not only on the variables $\left(t, q^{i}, x^{i}\right)$, but on "mixed" variables like $\left(t, q^{i}, y_{i}\right)$. The previous statements hold only locally. Lagrangian submanifolds are the geometric objects behind generating functions. Even when the generating functions develops singularities (caustics), these objects are well-defined, as it is pointed out in [1].

\section{Generating functions for Poisson Manifolds}

\subsection{Motivation}

In the previous section we have provided a method to look for (generally local) Poisson isomorphisms. Assume that we have the same Hamiltonian system as in the previous section, $\left(\mathbb{R} \times A^{*} G, \Pi_{\mathbb{R}}, H\right)$. Since any Lagrangian bisection $L$ of the symplectic groupoid $T^{*}(\mathbb{R} \times G)$ produces a Poisson isomorphism on the base, which we will denote by $\hat{L}: \mathbb{R} \times A^{*} G \rightarrow \mathbb{R} \times A^{*} G$, then the equations of motion $\frac{\partial}{\partial t}+X_{H}$ transform to another Hamiltonian equations (see Theorem 7 ). In this section we give an explicit formula for the new Hamiltonian, and therefore for the transformed Hamilton's equations. After that, we look for explicit coordinates where we can compute the Lagrangian submanifolds of interest. We are mainly interested in computing the perturbations, nearby Lagrangian submanifolds, of a very important Lagrangian submanifold, the submanifold of identities, $\epsilon(M)$, of the cotangent groupoid under consideration, $T^{*} G$. These Lagrangian submanifolds happen to be generally non-horizontal, even in the classical or LiePoisson settings, for the canonical projection $\pi_{G}$. We propose a local solution that allows us to develop our Poisson-integrators under the situations described in the previous sections. The reader familiar with the classical Hamilton-Jacobi theory should notice that we are describing here the geometric counterpart of the usually stated fact that Hamilton's equations for the Hamiltonian $H$ in the coordinates $\left(t, q^{i}, p_{i}\right)$, say

$$
\begin{aligned}
& \frac{d}{d t} q^{i}(t)=\frac{\partial H}{\partial p_{i}}\left(q^{i}(t), p_{i}(t)\right) \\
& \frac{d}{d t} p_{i}(t)=-\frac{\partial H}{\partial q^{i}}\left(q^{i}(t), p_{i}(t)\right)
\end{aligned}
$$


transform via a generating function $S$ to another system of Hamilton's equations is coordinates $\left(t, Q^{i}, P_{i}\right)$ for a new Hamiltonian function $K$

$$
\begin{aligned}
& \frac{d}{d t} Q^{i}(t)=\frac{\partial K}{\partial P_{i}}\left(Q^{i}(t)\right) \\
& \frac{d}{d t} P_{i}(t)=-\frac{\partial K}{\partial Q^{i}}\left(Q^{i}(t)\right)
\end{aligned}
$$

and the relation between the two Hamiltonians is given by

$$
\frac{\partial S}{\partial t}+H\left(t, q^{i}, p_{i}\right)=K\left(t, Q^{i}, P_{i}\right)
$$

\subsection{Fundamental Lemma}

The next lemma says how the time evolution, $\frac{\partial}{\partial t}$, transforms under the mapping $\hat{L}$. This is the hard part of the proof, since the evolution of the the Hamiltonian vector field $X_{H}$ is known because $\hat{L}$ is a Poisson mapping. Once more, we assume that the induced map is a global diffeomorphism, since the local case follows changing the domains and range of definition by the appropriate open sets.

Lemma 6. Let $L$ be a Lagrangian bisection of the symplectic groupoid $T^{*}(\mathbb{R} \times$ $G)$. Then the induced Poisson mapping, $\hat{L}: \mathbb{R} \times A^{*} G \rightarrow \mathbb{R} \times A^{*} G$, satisfies

$$
\hat{L}_{*}\left(\frac{\partial}{\partial t}\right)=\frac{\partial}{\partial t}+X_{\sigma_{2}^{*} e}
$$

where $\sigma_{2}=\left(\widetilde{\alpha_{\mathbb{R}} \mid L}\right)^{-1}$.

Proof: From now on we will write $\sigma_{1}=\left(\widetilde{\beta_{\mathbb{R}} \mid L}\right)^{-1}: \mathbb{R} \times A^{*} G \rightarrow L \subset T^{*}(\mathbb{R} \times G)$ and $\sigma_{2}=\left(\widetilde{\alpha_{\mathbb{R}} \mid L}\right)^{-1}: \mathbb{R} \times A^{*} G \rightarrow L \subset T^{*}(\mathbb{R} \times G)$. Consider the Hamiltonian $-\sigma_{1}^{*} e: \mathbb{R} \times A^{*} G \rightarrow \mathbb{R}$. Then the associated extended Hamiltonian is $\left(\sigma_{1}^{*} e\right)^{e}=$ $-{\widetilde{\beta_{\mathbb{R}}}}^{*}\left(\sigma_{1}^{*} e\right)+e$. Notice that the Lagrangian submanifold $L$ satisfies the HamiltonJacobi equation, that is, $\left(-{\widetilde{\beta_{\mathbb{R}}}}^{*}\left(\sigma_{1}^{*} e\right)+e\right)_{\mid L}=0$ and, so, Theorem 5 (First Step) holds using Remark 19. We conclude that

$$
\hat{L}_{*}\left(\frac{\partial}{\partial t}+X_{-\sigma_{1}^{*} e}\right)=\frac{\partial}{\partial t} .
$$

Now, since $\hat{L}$ is a Poisson automorphism, we have $\hat{L}_{*}\left(X_{\sigma_{1}^{*} e}\right)=X_{\left(\hat{L}^{-1}\right)^{*}\left(\sigma_{1}^{*} e\right)}$. It is easy to see that $\sigma_{1}^{*} e \circ \hat{L}^{-1}=\sigma_{2}^{*} e$ and thus

$$
\hat{L}_{*}\left(X_{\sigma_{1}^{*} e}\right)=X_{\sigma_{2}^{*} e} .
$$

Finally, adding equations (29) and (30) we get

$$
\hat{L}_{*}\left(\frac{\partial}{\partial t}\right)=\hat{L}_{*}\left(\frac{\partial}{\partial t}+X_{-\sigma_{1}^{*} e}+X_{\sigma_{1}^{*} e}\right)=\frac{\partial}{\partial t}+X_{\sigma_{2}^{*} e}
$$




\subsection{Main Result}

With the previous lemma at hand, we can easily prove the following theorem. We maintain the notations used in the previous lemma.

Theorem 7. Let $H: \mathbb{R} \times A^{*} G \rightarrow \mathbb{R}$ be a Hamiltonian function and $L \subset T^{*}(\mathbb{R} \times G)$ a Lagrangian bisection. The induced mapping $\hat{L}: \mathbb{R} \times A^{*} G \rightarrow \mathbb{R} \times A^{*} G$ satisfies

$$
\hat{L}_{*}\left(\frac{\partial}{\partial t}+X_{H}\right)=\frac{\partial}{\partial t}+X_{K}
$$

where $K=H \circ \hat{L}^{-1}+\sigma_{2}^{*} e$.

Proof: The proof is a consequence of Lemma 6. First notice that since $\hat{L}$ is Poisson, we have $\hat{L}_{*}\left(X_{H}\right)=X_{H \circ \hat{L}^{-1}}$. Combining this result with Lemma 6 we obtain

$$
\hat{L}_{\star}\left(\frac{\partial}{\partial t}+X_{H}\right)=\hat{L}_{*}\left(\frac{\partial}{\partial t}\right)+\hat{L}_{*}\left(X_{H}\right)=\frac{\partial}{\partial t}+X_{\sigma_{2}^{e}}+X_{H \circ \hat{L}^{-1}}=\frac{\partial}{\partial t}+X_{K} .
$$

Remark 20. Of course, the resemblances between the equations $\frac{\partial S}{\partial t}+H\left(t, q^{i}, p_{i}\right)=$ $K\left(t, Q^{i}, P_{i}\right)$ and $K=H \circ \hat{L}^{-1}+\sigma_{2}^{*} e$ are obvious. When $L=\operatorname{Im}(d S)$ the last equation reduces to a generalization of the first one

$$
K=H \circ \hat{L}^{-1}+\frac{\partial S}{\partial t} .
$$

In the pair groupoid case we recover the classical results.

It is natural to wonder which Poisson transformation on $\mathbb{R} \times A^{*} G$ are of the form $\hat{L}$ for some Lagrangian bisection $L \subset T^{*}(\mathbb{R} \times G)$. It is shown in the reference [13] that under some conditions $\left({\widetilde{\beta_{\mathbb{R}}}}^{-1}\left(\mu_{x}\right)\right.$ be connected for all $\left.\mu_{x} \in \mathbb{R} \times A^{*} G\right)$ one can find any Poisson transformation preserving the symplectic leaves of $\mathbb{R} \times A^{*} G$ by that procedure. It is also shown there, that the flow $\frac{\partial}{\partial t}+X_{H}$ induces a Lagrangian submanifold in $T^{*}(\mathbb{R} \times G)$ which satisfies the "strong" Hamilton-Jacobi equation in the sense that $H_{\mid L}^{e}=0$. In that way, that gives some existence results. Notice that it may happen that $L$ is not horizontal, i.e. it can not be written as $\operatorname{Im}(\gamma)$ for any closed 1 -form $\gamma$ on $\mathbb{R} \times G$. We will introduce this construction in the next subsection and show how to fix this problem.

\subsection{Generating the identity: non-free canonical transfor- mations}

\subsubsection{Motivation: Classical Case}

We start this subsection showing that even in the classical situation, described in Section 1.1.1, the identity transformation $\operatorname{id}_{T^{*} Q}: T^{*} Q \rightarrow T^{*} Q$ can not be 
4 GENERATING FUNCTIONS FOR POISSON MANIFOLDS

obtained through generating functions of the first type, i.e. $S\left(x^{i}, q^{i}\right)$, where $\left(q^{i}\right)$ and $\left(x^{i}\right)$ are coordinates in $Q$, the configuration manifold. Equivalently, the Lagrangian submanifold given by the identities is not horizontal for $\pi_{Q \times Q}$. In the language of [2] a canonical transformation is called free if it has a generating function of type I and non-free otherwise. The local coordinate description is all that we are going to need and, thus, we assume that $Q=\mathbb{R}^{n}$ and so $T^{*} Q=\mathbb{R}^{2 n}$ and consider global coordinates $\left(q^{i}, p_{i}\right)$. Doubling these coordinates we get a coordinate system for $T^{*}(Q \times Q)=\mathbb{R}^{4 n}$, say $\left(x^{i}, y_{i}, q^{i}, p_{i}\right)$. It is easy to see that the Lagrangian submanifold of the identities $L=\epsilon(M)$ is given by the set of points of the form

$$
L=\left\{\left(q^{i},-p_{i}, q^{i}, p_{i}\right) \text { such that } q^{i}, p_{i} \in \mathbb{R}\right\},
$$

or equivalently

$$
L=\left\{\left(x^{i}, y_{i}, q^{i}, p_{i}\right) \text { such that } q^{i}=x^{i},-p_{i}=y_{i} \in \mathbb{R}\right\} .
$$

Using the description of the source and the target it can be checked that $L$ induces the identity transformation. Obviously, it does not exists $S\left(x^{i}, q^{i}\right)$ such that $\operatorname{Im}(d S)=L$. In order to solve that, we introduce the following symplectomorphism.

$$
\begin{aligned}
& F:\left(\mathbb{R}^{4 n}, d x^{i} \wedge d y_{i}+d q^{i} \wedge d p_{i}\right) \longrightarrow\left(\mathbb{R}^{4 n}, d y_{i} \wedge d x^{i}+d q^{i} \wedge d p_{i}\right) \\
& \left(x^{i}, y_{i}, q^{i}, p_{i}\right) \quad \rightarrow \quad\left(\tilde{x}^{i}=x^{i}, \tilde{y}_{i}=-y_{i}, \tilde{q}^{i}=q^{i}, \tilde{p}_{i}=p_{i}\right)
\end{aligned}
$$

The main idea is that we interchanged the role of $x^{i}$ and $y_{i}$. Since symplectomorphisms conserve Lagrangian submanifolds, $\tilde{L}=F(L)$ is a Lagrangian submanifold in $\left(\mathbb{R}^{4 n}, d y_{i} \wedge d x^{i}+d q^{i} \wedge d p_{i}\right)$. The submanifold $\tilde{L}$ is explicitly given by

$$
\tilde{L}=\left\{\left(q^{i}, p_{i}, q^{i}, p_{i}\right) \text { such that } q^{i}, p_{i} \in \mathbb{R}\right\},
$$

We introduce a projection analogous to $\pi_{Q}$, which in general is only defined locally and in a non-canonical way

$$
\begin{aligned}
& \pi: \quad \mathbb{R}^{4 n} \quad \longrightarrow \mathbb{R}^{2 n} \\
& \left(x^{i}, y_{i}, q^{i}, p_{i}\right) \quad \rightarrow \quad \pi\left(x^{i}, y_{i}, q^{i}, p_{i}\right)=\left(y_{i}, q^{i}\right) .
\end{aligned}
$$

Now, $\pi: \mathbb{R}^{4 n} \rightarrow \mathbb{R}^{2 n}$ with the symplectic structure $\mathbb{R}^{4 n}, d q^{i} \wedge d p_{i}+d y_{i} \wedge d x^{i}$ is "the same as a cotangent bundle", but there $\tilde{L}$ is horizontal. We can consider the function $S\left(y_{i}, q^{i}\right)=q^{i} \cdot y_{i}$ and $\tilde{L}=\operatorname{Im}(d S)$. It is now obvious that we can use $F$ to turn non-horizontal Lagrangian submanifolds into horizontal ones. Using $F^{-1}$, once a generating function is obtained for the desired Lagrangian submanifold, we can describe a canonical transformation implicitly following the same pattern we use in (3). That is

$$
\tilde{L}=\left\{\left(\frac{\partial S}{\partial y_{i}}\left(y_{i}, q^{i}\right), y_{i}, q^{i}, \frac{\partial S}{\partial q^{i}}\left(y_{i}, q^{i}\right)\right) \text { such that } q^{i}, y_{i} \in \mathbb{R}\right\}
$$


and

$$
L=F^{-1}(\tilde{L})=\left\{\left(\frac{\partial S}{\partial y_{i}}\left(y_{i}, q^{i}\right),-y_{i}, q^{i}, \frac{\partial S}{\partial q^{i}}\left(y_{i}, q^{i}\right)\right) \text { such that } q^{i}, y_{i} \in \mathbb{R}\right\}
$$

that in general will induce the transformation analogous to (3)

$$
\frac{\partial S}{\partial y^{i}}\left(t, y_{i}, q^{i}\right)=x_{i}, \quad \frac{\partial S}{\partial q^{i}}\left(t, y_{i}, q^{i}\right)=p_{i} .
$$

which is usually called type $I I$ generating function. The expression above is found in most classical mechanics books, like $[2,16]$. In the particular case $S\left(y_{i}, q^{i}\right)=q^{i} \cdot y_{i},(32)$ reads

$$
y_{i}=p_{i}, \quad q^{i}=x^{i} .
$$

that is, we generated the identity.

Remark 21. The transformations (3), (32) are just the transformations defined by the corresponding Lagrangian bisections following Theorem 3 .

The previously described situation illustrates the importance of "projecting" Lagrangian submanifolds in the appropriate setting. While their geometry is very rich and powerful, whenever one is interesting in computations these Lagrangian submanifolds should be described by functions. Conditions on the Lagrangian submanifold, like the Hamilton-Jacobi equation $H_{\mid L}^{e}=0$, become PDEs that can be treated by the techniques of analysis. In the rest of this section we will describe analogous procedures to generate the identity in the three cases of interest: Lie algebras, action and Atiyah Lie algebroids. A general method is provided in the appendices.

\subsubsection{Lie Algebras}

Let $\mathfrak{g}$ a Lie algebra and $G$ a Lie group integrating it (not necessarily simplyconnected). Then the cotangent groupoid $T^{*} G \rightrightarrows \mathfrak{g}^{*}$ is endowed with the following two natural projections

$$
\begin{array}{ll}
\tilde{\alpha}: & T^{*} G \rightarrow \mathfrak{g}^{*} \\
& \left(\mu_{g}\right) \rightarrow \tilde{\alpha}\left(\mu_{g}\right)=J_{L}\left(\mu_{g}\right) \\
\tilde{\beta}: & T^{*} G \rightarrow \mathfrak{g}^{*} \\
& \left(\mu_{g}\right) \rightarrow \tilde{\beta}\left(\mu_{g}\right)=J_{R}\left(\mu_{g}\right) .
\end{array}
$$

This is the only structure needed, we will not use the algebraic properties of groupoids in this section. It is easy to check that the submanifold of identities $L=\epsilon\left(\mathfrak{g}^{*}\right)$ is given by the fiber $\pi_{G}^{-1}(e)$, where $e$ is the identity element. It is quite illustrative to notice that we have the opposite situation of an horizontal Lagrangian submanifold for $\pi_{G}$, the projection of $L$ is just $\{e\}$. This suggests that we should try to "project $L$ onto the fibers", not into $G$. In order to do that, take local coordinates around the identity $e$, say $\left(g^{i}\right), i=1, \ldots, n$ and let 
$\left(g^{i}, p_{i}\right)$ be the associated natural coordinates on $T^{*} G$. Assume for the sake of simplicity that $\left(g^{i}\right)(e)=0$. In those coordinates $L$ is given by

$$
L=\left\{\left(0, p_{i}\right) \text { such that } p_{i} \in \mathbb{R}\right\} .
$$

We introduce the, only locally defined, projection

$$
\begin{aligned}
& \pi:\left(g^{i}, p_{i}\right) \longrightarrow\left(p_{i}\right) \\
& \left(g^{i}, p_{i}\right) \quad \rightarrow \quad \pi\left(g^{i}, p_{i}\right)=\left(p_{i}\right)
\end{aligned}
$$

We think about the space $\left(g^{i}, p_{i}\right)$ with the symplectic form $d g^{i} \wedge d p_{i}$ and projection $\pi$ as a "cotangent bundle". Then we can consider $S\left(p^{i}\right)=0$ and $L$ is identified as the graph of the differential of this function

$$
L=\left\{\left(0, p_{i}\right) \text { such that } p_{i} \in \mathbb{R}\right\}=\left\{\left(\frac{\partial S}{\partial p_{i}}\left(p_{i}\right), p_{i}\right) \text { such that } p_{i} \in \mathbb{R}\right\} .
$$

The point of this construction is that nearby Lagrangian submanifolds will be described by this kind of functions as well. This is the keystone for the construction of our numerical methods in Section 6 and it solves a question by McLachlan and Scovel ([29]) that we mentioned in the introduction. Although this constructions are local, they permit us to parametrize the requested Lagrangian submanifolds, as it can be shown in our examples.

To finish this subsection, we illustrate how to deal with an example taken from [14] where it is shown that there is no way to find a generating function depending on coordinates on the base $S\left(g^{i}\right)$ for the identity $\operatorname{id}_{\mathfrak{g}^{*}}: \mathfrak{g}^{*} \rightarrow \mathfrak{g}^{*}$. How to generate the identity transformation was only known for quadratic Lie algebras, [4].

Example 1. Consider the group

$$
\operatorname{Train}(2)=\left\{\left(\begin{array}{ll}
a_{11} & a_{12} \\
0 & a_{22}
\end{array}\right) \in \operatorname{GL}(2, \mathbb{R})\right\} .
$$

We introduce coordinates

$$
\left(g^{1}, g^{2}, g^{3}\right) \rightarrow\left(\begin{array}{ll}
a_{11}=g^{1} & a_{12}=g^{2} \\
0 & a_{22}=g^{3}
\end{array}\right)
$$

defined for $\left(g^{1}, g^{2}, g^{3}\right) \in(\mathbb{R}-\{0\}) \times(\mathbb{R}-\{0\}) \times \mathbb{R}$. The product is given in local coordinates by $\left(g^{1}, g^{2}, g^{3}\right) \cdot\left(\tilde{g}^{1}, \tilde{g}^{2}, \tilde{g}^{3}\right)=\left(g^{1} \tilde{g}^{1}, g^{1} \tilde{g}^{2}+g^{2} \tilde{g}^{3}, g^{3} \tilde{g}^{3}\right)$. Let $\left(g^{1}, g^{2}, g^{3}, p_{1}, p_{2}, p_{3}\right)$ be natural coordinates in $T^{*} \operatorname{Train}(2)$ and a straightforward computation shows that

$$
\begin{aligned}
& \tilde{\alpha}\left(g^{1}, g^{2}, g^{3}, p_{1}, p_{2}, p_{3}\right)=J_{L}\left(g^{1}, g^{2}, g^{3}, p_{1}, p_{2}, p_{3}\right)=\left(p_{1} g^{1}+p_{2} g^{2}, p_{2} g^{3}, p_{3} g^{3}\right) \\
& \tilde{\beta}\left(g^{1}, g^{2}, g^{3}, p_{1}, p_{2}, p_{3}\right)=J_{R}\left(g^{1}, g^{2}, g^{3}, p_{1}, p_{2}, p_{3}\right)=\left(p_{1} x^{1}, p_{2} g^{1}, p_{2} g^{2}+p_{3} g^{3}\right) .
\end{aligned}
$$


In this case $\left(g^{1}, g^{2}, g^{3}\right)(e)=(1,0,1)$, so taking $S\left(p_{1}, p_{2}, p_{3}\right)=p_{1}+p_{3}$ which implies that

$$
\begin{aligned}
\operatorname{Im}(d S) & =\left\{\left(\frac{\partial S}{\partial p_{1}}(p), \frac{\partial S}{\partial p_{1}}(p), \frac{\partial S}{\partial p_{1}}(p), p_{1}, p_{2}, p_{3}\right) \text { such that } p_{i} \in \mathbb{R}\right\} \\
& =\left\{\left(1,0,1, p_{1}, p_{2}, p_{3}\right) \text { such that } p_{i} \in \mathbb{R}\right\} .
\end{aligned}
$$

Now we have

$$
\begin{aligned}
& \tilde{\alpha}\left(d S\left(p_{1}, p_{2}, p_{3}\right)\right)=\left(p_{1}, p_{2}, p_{3}\right) \\
& \tilde{\beta}\left(d S\left(p_{1}, p_{2}, p_{3}\right)\right)=\left(p_{1}, p_{2}, p_{3}\right),
\end{aligned}
$$

and equation (35) implies that the transformation induced by $\operatorname{Im}(d S)$ is the identity.

\subsubsection{Action Lie Algebroids}

Action Lie algebroids are discussed in Appendix B. The connection of these algebroids with semi-direct products is very remarkable, as these structures have proved their importance describing some of the dynamical systems relevant for geometric mechanics, see [25]. Assume that our action Lie algebroid, say $M \times \mathfrak{g}$, is integrable with the Lie groupoid $M \times G$ integrating it. Then $T^{*}(M \times G) \rightrightarrows M \times \mathfrak{g}^{*}$ is a symplectic Lie groupoid with source and target given by

$$
\begin{aligned}
& \tilde{\alpha}: \quad T^{*}(M \times G) \rightarrow M \times \mathfrak{g}^{*} \\
& \left(\mu_{q}^{1}, \mu_{g}^{2}\right) \quad \rightarrow \quad \tilde{\alpha}\left(\mu_{q}^{1}, \mu_{g}^{2}\right)=\left(q,-J\left(\mu_{q}^{1}\right)+J_{L}\left(\mu_{g}^{2}\right)\right) \\
& \tilde{\beta}: \quad T^{*}(M \times G) \rightarrow M \times \mathfrak{g}^{*} \\
& \left(\mu_{q}^{1}, \mu_{g}^{2}\right) \quad \rightarrow \quad \tilde{\beta}\left(\mu_{q}^{1}, \mu_{g}^{2}\right)=\left(q g, J_{R}\left(\mu_{g}^{2}\right)\right) .
\end{aligned}
$$

Where $J$ is the standar momentum mapping for lifted actions,

$$
J\left(\mu_{q}\right)(\xi)=\mu_{q}\left(\xi_{M}\right),
$$

$\xi_{M}$ is the infinitesimal generator associated to $\xi \in \mathfrak{g}$. On the other hand, the Lagrangian submanifold of identities is given by

$$
L=\epsilon\left(M \times \mathfrak{g}^{*}\right)=\left\{\left(0_{m}, \mu\right) \text { such that } m \in M, \mu \in \mathfrak{g}^{*}=T_{e}^{*} G\right\} .
$$

Taking local coordinates $\left(x^{i}\right)$ on an open set $U \subset M$, and $\left(g^{j}\right)$ coordinates on a neighbourhood of the identity on $G$, moreover, we assume that $g^{i}(e)=0$. We consider natural coordinates $\left(x^{i}, y_{i}, g^{j}, p_{j}\right)$ on $T^{*}(M \times G)$. In these coordinates $L$ is given by

$$
\left\{\left(x^{i}, 0,0, p_{j}\right) \text { such that } x^{i} \in U, p_{j} \in \mathbb{R}\right\}
$$

Arguing like in the previous sections, we get that we have to "project" onto the $\left(x^{i}, p_{j}\right)$-coordinates. We get a generating function $S\left(x^{i}, p_{j}\right)$ produces the Lagrangian submanifold, after a change of sign using a mapping analogous to $F$ in Section 4.4 .1

$$
\left\{\left(x^{i}, \frac{\partial S}{\partial x^{i}}\left(x^{i}, p_{j}\right), \frac{\partial S}{\partial p_{j}}\left(x^{i}, p_{j}\right), p_{j}\right), \text { such that } x^{i} \in U, p_{j} \in \mathbb{R}\right\}
$$

So taking $S\left(x^{i}, p_{j}\right)=$ constant gives us the desired generating function. 


\subsubsection{Atiyah Algebroids}

Atiyah algebroids are discussed in the appendix, their cotangent groupoids were already introduced in Section 2.3. We assume that $P \rightarrow M$ is trivial from the very beginning, as we are interested in the local description, so we have $T^{*}(M \times M \times G)$, and recall that

$$
\begin{aligned}
& \tilde{\alpha}: T^{*}(M \times M \times G) \longrightarrow T^{*} M \times \mathfrak{g}^{*} \\
& \left(\lambda_{m^{1}}, \mu_{m^{2}}, \nu_{g}\right) \rightarrow \tilde{\alpha}\left(\lambda_{m^{1}}, \mu_{m^{2}}, \nu_{g}\right)=\left(-\lambda_{m^{1}}, J_{R}\left(\nu_{g}\right)\right) \\
& \tilde{\beta}: \quad T^{*}(M \times M \times G) \longrightarrow T^{*} M \times \mathfrak{g}^{*} \\
& \left(\lambda_{m^{1}}, \mu_{m^{2}}, \nu_{g}\right) \rightarrow \tilde{\beta}\left(\lambda_{m^{1}}, \mu_{m^{2}}, \nu_{g}\right)=\left(\mu_{m^{2}}, J_{L}\left(\nu_{g}\right)\right) .
\end{aligned}
$$

The Lagrangian submanifold of identities is given by

$$
\left\{\left(-\lambda_{m}, \lambda_{m}, \nu\right) \text { such that } \lambda_{m} \in T^{*} M, \nu \in \mathfrak{g}^{*}\right\}
$$

It should be clear, looking at the expressions above, that this case is a combination of Section 4.4.1 and Section 4.4.2. Taking the union of the $\left(q^{i}, x^{i}\right)$ and $g^{j}$ introduced in the aforementioned sections, we get the coordinate system $\left(q^{i}, x^{i}, g^{j}\right)$ on $M \times M \times G$. Combining the arguments exposed there, a generating function of the form $S\left(q^{i}, x^{i}, p_{j}\right)$ is obtained in a straightforward manner. The $p_{j}$ in the previous expression are the momenta associated to the $g^{j}$.

\section{Local Existence of Solution}

Since we are dealing with a PDE one of our main tasks is to show, at least, local existence of solutions of the corresponding Hamilton-Jacobi equation. Our proof follows the construction of Ge in [13] to build a Lagrangian submanifold which satisfies the Hamilton-Jacobi equation, according to Remark 19. We go further because our cotangent groupoid structure allows us to show that, under certain initial value conditions, the aforementioned Lagrangian submanifold is horizontal, and then it happens to be the image of a closed 1-form. Thus, locally the graph of that 1-form is the differential of a function, $S$, which satisfies the Hamilton-Jacobi equation. The result follows after applying basic stability theory results. Our construction works for arbitrary Hamiltonian functions $H: \mathbb{R} \times A^{*} G \rightarrow \mathbb{R}$.

Before proving the results stated in the paragraph above, we generalize a classical and very popular result, the action given by a regular Lagrangian gives a type I solution of the Hamilton-Jacobi equation. This result establish the link of the Hamilton-Jacobi theory with the variational integrators.

\subsection{Existence of type I solutions for Lagrangian systems}

When the Hamiltonian system under consideration comes from a (hyper-)regular Lagrangian, there exists a local solution, given by the action functional

$$
S\left(t, q^{i}, x^{i}\right)=\int_{0}^{t} L(\dot{c}(s)) d s
$$


where $c(t)$ is the unique curve satisfying the Euler-Lagrange equations with the boundary conditions

$$
\begin{aligned}
& c(0)=\left(q^{i}\right), \\
& c(t)=\left(x^{i}\right) .
\end{aligned}
$$

The details of the construction can be found in the references $[2,16,26]$. For useful information about Lagrangian dynamics on Lie algebroids we recommend $[21,23,33]$. We extend this result to our setting, following an auxiliary construction introduced in [22,33]. The vector bundle $\pi: \operatorname{ker}(T \alpha) \rightarrow G$, where $\pi$ is the natural projection, has a Lie algebroid structure given by

1. The anchor $T \beta_{\mid \operatorname{ker}(T \alpha)}: \operatorname{ker}(T \alpha) \rightarrow T G$.

2. The Lie bracket is just the restriction of the Lie bracket on vector fields.

This Lie algebroid is related to $\tau: A G \rightarrow M$ through the mapping below, which happens to be a Lie algebroid morphism

$$
\begin{aligned}
\Psi: \operatorname{ker}(T \alpha) & \longrightarrow A \\
X_{g} & \rightarrow \Psi\left(X_{g}\right)=d L_{g^{-1}}\left(X_{g}\right) .
\end{aligned}
$$

Given a Lagrangian function in the Lie algebroid $L: A G \rightarrow \mathbb{R}$, we can consider the induced Lagrangian dynamics in $\operatorname{ker}(T \alpha)$ by $L \circ \Psi$. The relation between the two systems is given by the following theorem.

Theorem 8 (See [33], Theorem 4.5). Let $\Psi: A \rightarrow B$ be a morphism of Lie algebroids, and let $L$ be a regular Lagrangian on $B$. If $\Psi$ is an isomorphism on each fiber, then the image under $\Psi$ of any solution of Lagrange's equations for $L \circ \Psi$ is a solution of Lagrange's equations for $L$.

The main observation is that the Lagrangian dynamics can be interpreted as a "parametrized" version of the usual Lagrangian theory on tangent bundles. An easy computation shows that for any initial condition $X_{g} \in \operatorname{ker}(T \alpha)$ the source does not evolve, that is $\alpha \circ \pi\left(\varphi_{t}^{X_{\text {Low }}}\left(X_{g}\right)\right)=\alpha(g)$, where $\varphi_{t}^{X_{L \circ \Psi}}$ is the flow of the vector field $X_{L \circ \Psi}$ whose trajectories are the solutions of the EulerLagrange equations. That explains why we can think about the Lagrangian system $(\operatorname{ker}(T \alpha), L \circ \Psi)$ as a "bundle of Lagrangian systems" $\left(T \alpha^{-1}(m),(L \circ\right.$ $\left.\Psi)_{\mid \alpha^{-1}(m)}\right)$ parametrized by $m \in M$. From now on we use $L_{m}=(L \circ \Psi)_{\mid \alpha^{-1}(m)}$. Of course, given $X_{g}$ the flow produced by the two Lagrangian systems, $\varphi_{t}^{X_{L \circ \Psi}}$ and $\varphi_{t}^{X_{L_{m}}}$ with initial condition $X_{g}$ coincides, and that concludes our observation. We can reduce now our problem to the classical tangent bundle case. Given $g$, close enough to the identity $\epsilon(\alpha(g))$ and a time $t$ small enough, there exists an initial condition $X \in \operatorname{ker}(T \alpha)_{\epsilon(\alpha(g))}=T \alpha^{-1}(\alpha(g))$ such that $\varphi_{t}^{X_{L \circ \Psi}}(X)=$ $g$, just by direct application of the classical result on tangent bundles, to the system $\left(T \alpha^{-1}(\alpha(g)), L_{\alpha(g)}\right)$. Since this constructions only hold locally, close 
enough in this section can be understood with the usual euclidean norm in local coordinates, for instance. The classical theory also says that we can define

$$
S(t, g)=\int_{0}^{t} L\left(\varphi_{s}^{X_{L \circ \Psi}}(X)\right) d s
$$

in an appropriate open set, that we do not describe here, for all $g \in \alpha^{-1}(\alpha(g))$ such that $g$ is close to $\epsilon(\alpha(g))$.

Remark 22. The classical situation corresponds to the pair groupoid case. There $G=Q \times Q$ and $\operatorname{ker}(T \alpha) \equiv\{0\} \times T Q$ and $\Psi\left(0, X_{q}\right)=\left(X_{q}\right)$. So for any $q^{1} \in Q$ we have the Lagrangian system $L_{q^{1}}\left(X_{q^{2}}\right)=L\left(X_{q^{2}}\right)$. Thus, given $q^{1}$ close to $q^{2}$, which is equivalent to saying $\left(q^{1}, q^{2}\right)$ ( $g$ in the previous discussion) close to $\left(q^{1}, q^{1}\right)(\epsilon(\alpha(g))$ in the previous discussion) there exists a unique path satisfying the Euler-Lagrange equations joining them. Notice that given an initial condition $\left(0_{q^{1}}, X_{q^{2}}\right)$ the source, $q^{1}$, does not evolve.

The Legendre transformations, $\mathbb{F}(L \circ \Psi)$ and $\mathbb{F} L$, make the diagram below commutative

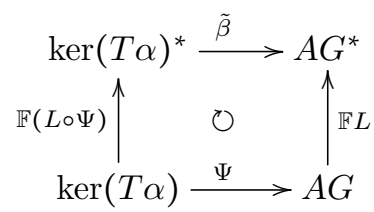

The corresponding Hamiltonians, say $H_{L \circ \Psi}: \operatorname{ker}(T \alpha) \rightarrow \mathbb{R}$ and $H_{L}: A^{*} G \rightarrow \mathbb{R}$ satisfy $H_{L \circ \Psi}=H_{L} \circ \tilde{\beta}$. Once more, direct application of the tangent bundle case shows that $S$ satisfies

$$
\frac{\partial S}{\partial t}+H\left(\tilde{\beta} \circ \frac{\partial S}{\partial g}\right)=0
$$

Remark 23. The previous construction shows the link of our theory with variational integrators, in regard of equation (38). The Hamilton-Jacobi equation can be understood in this way as a computation of the exact discrete Lagrangian.

Remark 24. The same proof with the obvious modifications holds in the timedependent case.

\subsection{Method of Characteristics}

We give the procedure below to show existence of solutions of our HamiltonJacobi equation. Actually, what we use is the classical method of characteristics adapted to this situation.

Assume that we are dealing with the general setting, that is, our Hamiltonian is defined in the dual bundle of an integrable Lie algebroid, $H: \mathbb{R} \times A^{*} G \rightarrow \mathbb{R}$. Let $L_{0}=\epsilon\left(A^{*} G\right) \subset T^{*} G$ be the Lagrangian submanifold given by the units of the cotangent groupoid, and $L_{1}=\{(0,0)\} \times L_{0} \subset T^{*}(\mathbb{R} \times G)$. Let $X_{H^{e}}$ be the Hamiltonian vector field of the associated extended Hamiltonian, defined in previous sections and $\varphi^{X_{H}}(t, p)$ its flow. Consider the immersion below

$$
\begin{aligned}
& i: \mathbb{R} \times L_{1} \longrightarrow T^{*}(\mathbb{R} \times G) \\
& (t, p) \quad \rightarrow \quad \varphi^{X_{H^{e}}}(t, p),
\end{aligned}
$$


a simple computation shows that its image, $L$, is a Lagrangian submanifold, embedded Lagrangian submanifold when restricted to small open sets. The fact that, by construction, this Lagrangian submanfild is tangent to $X_{H^{e}}$ implies that $d H^{e}$ restricted to this manifold is 0 , then $H^{e}$ is constant in any connected component. For small $t$, it is obvious that $L_{t}=\varphi^{X_{H}{ }^{e}}\left(L_{1}\right)$ will be a bisection, al least restricting ourselves to an open subset of $L_{t}$. Summarizing, we get a Lagrangian bisection such that $H_{\mid L}^{e}$ is constant.

\subsubsection{Local Solution}

In this section we give an abstract argument which justifies our choice of local coordinates (see Section 4.4). We just sketch the proof, as it is straightforward. Imagine that we are in the setting of the previous Section 5.2 , and that there exist a projection, which may be only locally defined, $\pi: T^{*} G \rightarrow B$ such that $L_{0}$ is horizontal. We are assuming that $\pi: T^{*} G \rightarrow B$ can be, at least locally, identified with a cotangent bundle, see Section 4.4 for an illustration. Then, maybe restricting to an open set of $L$, for $t \in \mathbb{R}$ small enough, $L$ is horizontal for $\left(\operatorname{id}_{\mathbb{R}}, \pi\right): T^{*}(\mathbb{R} \times G) \rightarrow \mathbb{R} \times B$, defined as $\left(\operatorname{id}_{\mathbb{R}}, \pi\right)\left(t, e, \mu_{g}\right)=(t, g)$, as an immediate application of stability of submersions. Since the argument is local, this is a consequence of the implicit function theorem. Making some abuse of notation, let us call this horizontal submanifold (restriction of $L$ to an open set) $L$ as well. This implies that $L$ is a Lagrangian submanifold in $T^{*}(\mathbb{R} \times G)$ and so, locally again, there exists $S$, defined using the mappings introduced in previous sections, such that $\operatorname{Im}(d S)=L$, and so we can guarantee local existence of solution. The projection $\pi$ has to be replaced by the appropriate mapping in each setting. For Lie algebras, action Lie algebroids and Atiyah algebroids, the existence of this map comes from Section 4.4.

\section{Examples}

Here we develop our applications to numerical methods. The general procedure is an improvement of the one outlined in [5]. It works for Lie algebras, action Lie algebroids and Atiyah Lie groupoids in a straightforward way, but it can be generalized to other settings.

\subsection{General Procedure}

1. Start with a Hamiltonian system in the dual of an integrable Lie algebroid $\left(\mathbb{R} \times A^{*} G, \Pi_{\mathbb{R}}, H\right)$.

2. Construct coordinates where the identity can be generated, using Section 4.4, and obtain the Hamilton-Jacobi equation

$$
\frac{\partial S}{\partial t}+H\left(t, \tilde{\beta} \circ \frac{\partial S}{\partial g}\right)=0
$$

in those coordinates. 
3. Approximate the solution taking the Taylor series in $t$ of $S$ up to order $k$, $S(t, g)=\sum_{i=0}^{k} S_{i}(t, g) t^{i} / i !+\mathcal{O}\left(t^{k+1}\right)$, where $S_{0}$ is the generating function of the identity.

4. The equations for the $S_{i}, i \geq 1$ can be solved recursively. For instance, in the case of Lie algebras we get for the three first terms

- $\mathbf{S}_{\mathbf{0}}\left(p^{i}\right)=0$.

- $\mathbf{S}_{\mathbf{1}}\left(p^{i}\right)+H\left(t, \frac{\partial S_{0}}{\partial p^{i}}, p^{i}\right)=0$.

- $\mathbf{S}_{\mathbf{2}}\left(p^{i}\right)+\frac{\partial H}{\partial t}\left(t, \frac{\partial S_{0}}{\partial p^{i}}, p^{i}\right)+\frac{\partial H}{\partial g^{i}}\left(t, \frac{\partial S_{0}}{\partial p^{j}}, p^{i}\right) \frac{\partial S_{1}}{\partial p^{i}}=0$

Each term can be obtained from the previous one by differentiating with respect to $t$ and evaluating at $t=0$.

5. Collecting all the terms obtained up to order $k, S^{k}=\sum_{i=0}^{k} S_{i} t^{i} / i$ !, we get an approximation of the solution of the Hamilton-Jacobi equation. It is easy to see that the transformation induced implicitly $\mu_{m^{1}}^{1} \rightarrow \mu_{m^{2}}^{2}, \mu_{m^{i}}^{i} \in A^{*} G$ by

$$
\tilde{\alpha} \circ d S^{k}(t, g)=\mu_{m^{1}}^{1} \quad \tilde{\beta} \circ d S^{k}(t, g)=\mu_{m^{2}}^{2}
$$

transforms the system to equilibrium up to order $k$. That is, it satisfies

$$
\frac{\partial S}{\partial t}+H\left(t, \tilde{\beta} \circ \frac{\partial S}{\partial g}\right)=\mathcal{O}\left(t^{k+1}\right) .
$$

Application of Theorem 7 gives that the transformation is an approximation of order $k$ of the flow and so the numerical method obtained by fixing a time-step, say $h$, is of order $k$.

We present now the results obtained after applying this scheme to different situations.

\subsection{Rigid Body}

The rigid body is one of the benchmarks of Lie-Poisson integrators. We show here the performance of the integrators described above. The configuration space for the rigid body (disregarding translations) is the Lie group $G=S O(3)$. The matrix $R \in S O(3)$ gives the configuration of the sphere as a rotation with respect to a reference configuration where its principal axes of inertia are aligned with the coordinate axes of an inertial system. Consider also a second system of coordinates fixed to the rotating body and aligned with the principal axes of inertia. We identify the Lie algebra $\mathfrak{s o}(3)$ with $\mathbb{R}^{3}$ using the isomorphism $\hat{:} \mathbb{R}^{3} \rightarrow \mathfrak{s o}(3)$ given by

$$
\hat{x}=\left(\begin{array}{ccc}
0 & -x_{3} & x_{2} \\
x_{3} & 0 & -x_{1} \\
-x_{2} & x_{1} & 0
\end{array}\right)
$$


As is usual, the angular velocity in body coordinates is denoted by $\Omega$, and $\hat{\Omega}=$ $R^{-1} \dot{R}$. The moment of inertia tensor in body coordinates is $\mathbb{I}=\operatorname{diag}\left(I_{1}, I_{2}, I_{3}\right)$. Also, the body angular momentum is $\Pi=\mathbb{I} \Omega$, so in principal axes, $\Pi=\left(\Pi_{1}, \Pi_{2}, \Pi_{3}\right)=$ $\left(I_{1} \Omega_{1}, I_{2} \Omega_{2}, I_{3} \Omega_{3}\right)$. The Hamiltonian in these variables is

$$
h=\frac{1}{2}\left(\frac{\Pi_{1}^{2}}{I_{1}}+\frac{\Pi_{2}^{2}}{I_{2}}+\frac{\Pi_{3}^{2}}{I_{3}}\right)
$$

which we regard as a function $h: \mathfrak{s o}(3)^{*} \rightarrow \mathbb{R}$. Note that if we regard $S O(3)$ as a Lie groupoid, then the dual Lie algebroid $A^{*} G$ is just $\mathfrak{s o}^{*}(3)$. We use the Cayley map cay: $\mathfrak{s o}(3) \rightarrow S O(3)$,

$$
\operatorname{cay}(\hat{\omega})=I_{3}+\frac{4}{4+\|\omega\|^{2}}\left(\hat{\omega}+\frac{\hat{\omega}^{2}}{2}\right)
$$

where $I_{3}$ is the $3 \times 3$ identity matrix (see [11]) to define local coordinates near the group identity. This gives a trivialization of the cotangent Lie groupoid $T^{*} S O(3)$ near the identity. The corresponding source and target maps, which map $T^{*} S O(3)$ into $\mathfrak{s o}(3)^{*}$, become locally $\tilde{\alpha}_{\text {cay }}, \tilde{\beta}_{\text {cay }}: \mathbb{R}^{3} \times\left(\mathbb{R}^{3}\right)^{*} \rightarrow \mathbb{R}^{3}$, and are given by

$$
\begin{gathered}
\tilde{\alpha}_{\text {cay }}\left(x, y, z, p_{x}, p_{y}, p_{z}\right)=\left(\begin{array}{c}
\left(\frac{x^{2}}{4}+1\right) p_{x}+\left(\frac{x y}{4}+\frac{z}{2}\right) p_{y}+\left(\frac{x z}{4}-\frac{y}{2}\right) p_{z} \\
\left(\frac{x y}{4}-\frac{z}{2}\right) p_{x}+\left(\frac{y^{2}}{4}+1\right) p_{y}+\left(\frac{y z}{4}+\frac{x}{2}\right) p_{z} \\
\left(\frac{x z}{4}+\frac{y}{2}\right) p_{x}+\left(\frac{y z}{4}-\frac{x}{2}\right) p_{y}+\left(\frac{z^{2}}{4}+1\right) p_{z}
\end{array}\right), \\
\tilde{\beta}_{\text {cay }}\left(x, y, z, p_{x}, p_{y}, p_{z}\right)=\left(\begin{array}{c}
\left(\frac{x^{2}}{4}+1\right) p_{x}+\left(\frac{x y}{4}-\frac{z}{2}\right) p_{y}+\left(\frac{x z}{4}+\frac{y}{2}\right) p_{z} \\
\left(\frac{x y}{4}+\frac{z}{2}\right) p_{x}+\left(\frac{y^{2}}{4}+1\right) p_{y}+\left(\frac{y z}{4}-\frac{x}{2}\right) p_{z} \\
\left(\frac{x z}{4}-\frac{y}{2}\right) p_{x}+\left(\frac{y z}{4}+\frac{x}{2}\right) p_{y}+\left(\frac{z^{2}}{4}+1\right) p_{z}
\end{array}\right) .
\end{gathered}
$$

The numerical method corresponding to a given truncation of the function $S$ gives the evolution of $\Pi \in A^{*} G=\mathfrak{s o}^{*}(3)$. We have run simulations using truncations of $S$ up to order 8 , for a rigid body with $\mathbb{I}=(0.81,1,0.21)$. We have used $\Pi_{0}=(1.5,0.1,0)$ as the initial value, which makes the body rotate near the middle (unstable) axis. The total run time was $T=5$, in which the body makes one "tumbling" motion, with decreasing values for the time-step $h$ (encoded as the variable $t$ in the function $S$ ). In Figure 5 we plot the norm of the global error, as a distance in $\mathbb{R}^{3}$, with respect to a Runge-Kutta simulation of the Euler equation $\dot{\Pi}=\Pi \times \Omega([24])$. Error values below $10^{-12}$ are not plotted due to inaccuracies caused by roundoff errors. For the rigid body, the terms with even orders in the expansion of $S$ are zero. 

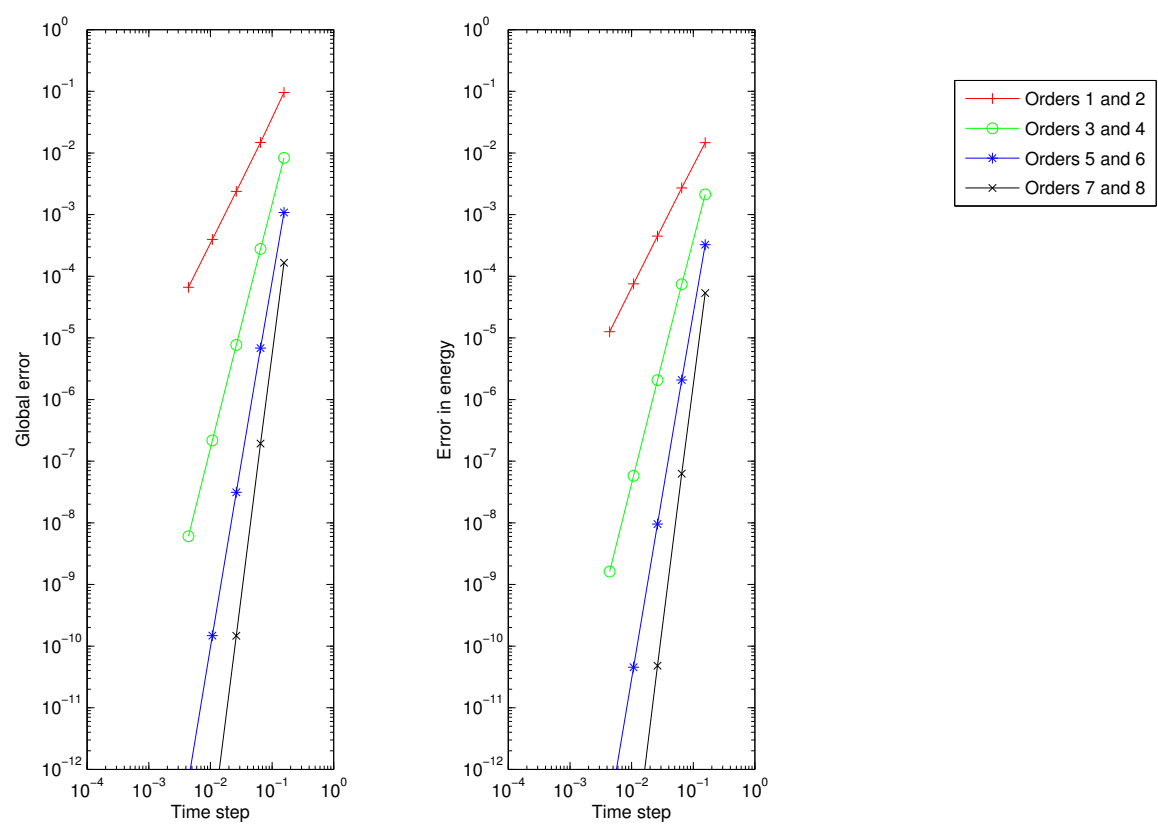

Figure 5: Errors for the rigid body simulations.

\subsection{Heavy Top}

As a concrete example of a system on a transformation Lie groupoid we consider the heavy top. This example is modeled on the action groupoid $\tau: S^{2} \times \mathfrak{s o}(3) \rightarrow S^{2}$ with Hamiltonian

$$
H(\Gamma, \Pi)=\frac{1}{2} \Pi \cdot I^{-1} \Pi+m g l \Gamma \cdot \mathbf{e},
$$

where $\Pi \in \mathbb{R}^{3} \simeq \mathfrak{s o}^{*}(3)$ is the angular momentum, $\Gamma$ is the direction opposite to the gravity and $\mathbf{e}$ is a unit vector in the direction from the fixed point to the center of mass, all them expressed in a frame fixed to the body. The constants $m, g$ and $l$ are respectively the mass of the body, the strength of the gravitational acceleration and the distance from the fixed point to the center of mass. The matrix $I$ is the inertia tensor of the body (see [21]).

In this case the Lie groupoid under consideration, $G$, is the action groupoid $S^{2} \times \mathfrak{s o}(3)$, whose Lie algebroid is $S^{2} \times \mathfrak{s o}^{*}(3)$. The source and target maps are 
given by

$$
\begin{aligned}
& \tilde{\alpha}_{\text {cay }}\left(a_{1}, a_{2}, a_{3}, x, y, z, p_{a_{1}}, p_{a_{2}}, p_{a_{3}}, p_{x}, p_{y}, p_{z}\right) \\
& =\left(\begin{array}{c}
a_{1} \\
a_{2} \\
a_{3} \\
\left(\frac{x^{2}}{4}+1\right) p_{x}+\left(\frac{x y}{4}-\frac{z}{2}\right) p_{y}+\left(\frac{x z}{4}+\frac{y}{2}\right) p_{z}+a_{2} p_{a_{3}}-a_{3} p_{a_{2}} \\
\left(\frac{x y}{4}+\frac{z}{2}\right) p_{x}+\left(\frac{y^{2}}{4}+1\right) p_{y}+\left(\frac{y z}{4}-\frac{x}{2}\right) p_{z}-a_{1} p_{a_{3}}+a_{3} p_{a_{1}} \\
\left(\frac{x z}{4}-\frac{y}{2}\right) p_{x}+\left(\frac{y z}{4}+\frac{x}{2}\right) p_{y}+\left(\frac{z^{2}}{4}+1\right) p_{z}+a_{1} p_{a_{2}}-a_{2} p_{a_{1}}
\end{array}\right) \\
& \text { and } \\
& \tilde{\beta}_{\text {cay }}\left(a_{1}, a_{2}, a_{3}, x, y, z, p_{a_{1}}, p_{a_{2}}, p_{a_{3}}, p_{x}, p_{y}, p_{z}\right) \\
& =\left(\begin{array}{c}
\frac{\left(x^{2}-y^{2}-z^{2}+4\right) a_{1}}{x^{2}+y^{2}+z^{2}+4}+\frac{(2 y x+4 z) a_{2}}{x^{2}+y^{2}+z^{2}+4}+\frac{(2 z x-4 y) a_{3}}{x^{2}+y^{2}+z^{2}+4} \\
\frac{\left(2 y x-4 z a_{1}\right.}{x^{2}+y^{2}+z^{2}+4}+\frac{\left(-x^{2}+y^{2}-z^{2}+4\right) a_{2}}{x^{2}+y^{2}+z^{2}+4}+\frac{(2 z y+4 x) a_{3}}{x^{2}+y^{2}+z^{2}+4} \\
\frac{(2 z x+4 y) a_{1}}{x^{2}+y^{2}+z^{2}+4}+\frac{(2 z y-4 x) a_{2}}{x^{2}+y^{2}+z^{2}+4}+\frac{\left(-x^{2}-y^{2}+z^{2}+4\right) a_{3}}{x^{2}+y^{2}+z^{2}+4} \\
\left(\frac{x^{2}}{4}+1\right) p_{x}+\left(\frac{x y}{4}+\frac{z}{2}\right) p_{y}+\left(\frac{x z}{4}-\frac{y}{2}\right) p_{z} \\
\left(\frac{x y}{4}-\frac{z}{2}\right) p_{x}+\left(\frac{y^{2}}{4}+1\right) p_{y}+\left(\frac{y z}{4}+\frac{x}{2}\right) p_{z} \\
\left(\frac{x z}{4}+\frac{y}{2}\right) p_{x}+\left(\frac{y z}{4}-\frac{x}{2}\right) p_{y}+\left(\frac{z^{2}}{4}+1\right) p_{z}
\end{array}\right)
\end{aligned}
$$
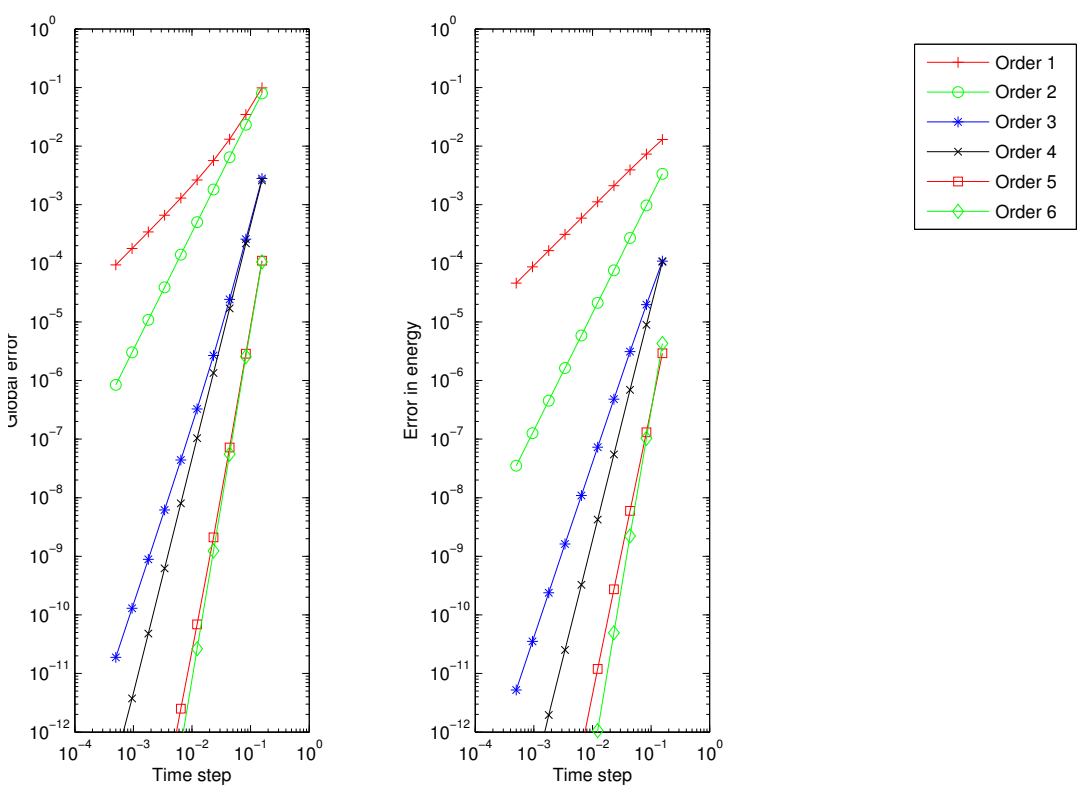

Figure 6: Errors for the heavy top simulations. 
We have run simulations using truncations of $S$ up to order 8 and the results are collected in Figure 6. The initial condition was

$$
\left(\Gamma_{0}, \Pi_{0}\right)=((0.5,0.5,-0.5) /\|(0.5,0.5,-0.5)\|,(0.1,-1,2))
$$

and the parameters $e=(0.1,0.2,0.5) /\|(0.1,0.2,0.5)\|, I=\operatorname{diag}(1,1.5,2), m=0.1$, $g=9.8$ and $l=0.2$.

\section{Conclusions and Remarks}

In this paper we developed a Hamilton-Jacobi theory for certain class of linear Poisson structures which happens to be general enough to include the systems important for classical mechanics. As a practical application we present an improvement of some Poisson numerical methods, previously introduced by Channell, Ge, Marsden and Scovel among others. There are still several issues to be exploited, we list some of them below.

1. Simplification of the Hamilton-Jacobi equations using Casimirs: In this paper we only treated some numerical methods as examples, but it is our belief that applications of our results are very promising for analytic integration of Hamilton's equations. This should not be surprising, as the classical Hamilton-Jacobi theory has proved to be one of the most powerful tools for analytic integration, see Arnold's quote in Section 1. In this regard, Casimirs should play a role, based on the following observation. If $H: A^{*} G \rightarrow \mathbb{R}$ is the Hamiltonian under consideration, and $C: A^{*} G \rightarrow \mathbb{R}$ is a Casimir, then $X_{H}=X_{H+\lambda C}$, where $\lambda$ is a constant. Nonetheless, the Hamilton-Jacobi equations for $H$ and for $H+\lambda C$ could be very different. As a simple but illustrative application of this fact, we present here an application to the computation of the rigid body when two moments of inertia are equal. It is remarkable that in [27] the author uses a similar procedure to obtain explicit Lie-Poisson integrators. For more information about that relation see point 3 below.

Example 2. Let $G$ be the Lie group $S O(3)$ and $0<\varphi<2 \pi, 0<\theta<\pi, 0<$ $\psi<2 \pi$ be the Euler angles, defined following [26]. They form a coordinate chart, although not including the identity. The source and target of the cotangent groupoid read in the associated cotangent coordinates

$$
\begin{gathered}
\tilde{\alpha}\left(\varphi, \psi, \theta, p_{\varphi}, p_{\psi}, p_{\theta}\right) \rightarrow\left(\begin{array}{c}
\Pi_{1}=\left(\left(p_{\psi}-\cos (\theta) p_{\varphi}\right) \sin (\varphi)+\cos (\varphi) \sin (\theta) p_{\theta}\right. \\
\Pi_{2}=\left(\cos (\theta) p_{\varphi}-p_{\psi}\right) \cos (\varphi)+\sin (\theta) \sin (\varphi) p_{\theta} \\
\Pi_{3}=p_{\varphi}
\end{array}\right) \\
\tilde{\beta}\left(\varphi, \psi, \theta, p_{\varphi}, p_{\psi}, p_{\theta}\right) \rightarrow\left(\begin{array}{c}
\Pi_{1}=\left(p_{\varphi}-p_{\psi} \cos (\theta)\right) \sin (\psi)+p_{\theta} \sin (\theta) \cos (\psi) \\
\Pi_{2}=\left(p_{\varphi}-p_{\psi}\right) \cos (\theta) \cos (\psi)-p_{\theta} \sin (\theta) \sin (\psi) \\
\Pi_{3}=p_{\psi}
\end{array}\right)
\end{gathered}
$$

The Lagrangian submanifold

$$
L=\left\{\left(\pi, \pi / 2, \pi / 2, p_{\varphi}, p_{\theta}, p_{\psi}\right) \text { such that } p_{\varphi}, p_{\psi}, p_{\theta}, \in \mathbb{R}\right\}
$$


generates the identity trasformation, and following our previous construction we can give the generating function

$$
S\left(p_{\varphi}, p_{\theta}, p_{\psi}\right)=\pi p_{\varphi}+1 / 2 \pi p_{\theta}+1 / 2 \pi p_{\psi} .
$$

The Hamiltonian of the rigid body dynamics, when two moments of inertia are equal, and the Casimir is given by

$$
\begin{aligned}
& H\left(\Pi_{1}, \Pi_{2}, \Pi_{3}\right)=\frac{1}{2}\left(\frac{\Pi_{1}^{2}}{I}+\frac{\Pi_{2}^{2}}{I}+\frac{\Pi_{3}^{2}}{I^{\prime}}\right), \\
& C\left(\Pi_{1}, \Pi_{2}, \Pi_{3}\right)=\Pi_{1}^{2}+\Pi_{2}^{2}+\Pi_{3}^{2} .
\end{aligned}
$$

The dynamics of the Hamiltonians $H$ and $H^{\prime}=H+\frac{1}{2 I} C$ are equal, since $C$ is a Casimir

$$
X_{H^{\prime}}\left(\Pi_{1}, \Pi_{2}, \Pi_{3}\right)=\left(\frac{I-I^{\prime}}{I I^{\prime}} \Pi_{2} \Pi_{3}\right) \partial \Pi_{1}+\left(\frac{I^{\prime}-I}{I I^{\prime}} \Pi_{1} \Pi_{3}\right) \partial \Pi_{2}+0 \partial \Pi_{3} .
$$

Nonetheless, the Hamiltonians $H$ and $H^{\prime}$ are very different as functions, that implies that their $\tilde{\beta}$-pullback are quite disparate as well

$$
\begin{aligned}
H \circ \tilde{\beta} & =\frac{1}{2}\left(\frac{\left[\left(p_{\varphi}-p_{\psi} \cos (\theta)\right) \sin (\psi)+p_{\theta} \sin (\theta) \cos (\psi)\right]^{2}}{I \sin ^{2}(\theta)}\right. \\
& \left.+\frac{\left[\left(p_{\varphi}-p_{\psi} \cos (\theta)\right) \cos (\psi)+p_{\theta} \sin (\theta) \sin (\psi)\right]^{2}}{I \sin ^{2}(\theta)}+\frac{p_{\psi}^{2}}{I^{\prime}}\right)
\end{aligned}
$$

while

$$
H^{\prime} \circ \tilde{\beta}=\frac{p_{\psi}^{2}}{I^{\prime}} .
$$

This fact should have implications in our Hamilton-Jacobi theory, and this is the case as we are going to show. A solution of the Hamilton-Jacobi equation using the Hamiltonian $H^{\prime}$

$$
\begin{aligned}
\frac{\partial S}{\partial t}\left(p_{\varphi}, p_{\theta}, p_{\psi}\right) & +\left(H^{\prime} \circ \tilde{\beta}\right)\left(\frac{\partial S}{\partial p_{\varphi}}, \frac{\partial S}{\partial p_{\theta}}, \frac{\partial S}{\partial p_{\psi}}, p_{\varphi}, p_{\theta}, p_{\psi}\right)=0 \\
& \Leftrightarrow \frac{\partial S}{\partial t}\left(p_{\varphi}, p_{\theta}, p_{\psi}\right)+\frac{p_{\psi}^{2}}{I^{\prime}}=0
\end{aligned}
$$

is given by direct inspection

$$
S\left(t, p_{\varphi}, p_{\theta}, p_{\psi}\right)=\frac{p_{\psi}^{2}}{I^{\prime}} t+S_{0}\left(p_{\varphi}, p_{\theta}, p_{\psi}\right),
$$

where $S_{0}$ is the initial condition, which is fixed to get the identity, so finally

$$
S\left(t, p_{\varphi}, p_{\theta}, p_{\psi}\right)=\frac{p_{\psi}^{2}}{I^{\prime}} t+\pi p_{\varphi}+1 / 2 \pi p_{\theta}+1 / 2 \pi p_{\psi} .
$$


For the sake of simplicity we chose to integrate the easiest term of the Hamiltonian, but the other terms are solvable in a similar fashion. On the other hand, the Hamilton-Jacobi equation for the Hamiltonian $H$ does not seem to be solvable in an obvious way. Although very elementary, this example shows that Casimirs can be used to simplify the Hamilton-Jacobi equation. A systematic development of these ideas should provide means to integrate the Hamilton-Jacobi equation. These ideas are exclusive of the Poisson setting, as the Casimirs are trivial for symplectic structures.

2. Improvement of the numerical methods: The numerical methods that we present here are very general and conserve the geometry very well, but they are not very efficient from the computational viewpoint. Our aim in this paper was to show how to use our results, more that giving optimized numerical methods. Nonetheless, there is still a lot of room to improve these methods, as it has already been shown in [3, 4, 9, 20, 29] in the symplectic and Lie-Poisson case, where they give recipes to construct higher order approximations and reduce the computational cost. Those improvements can be applied in a straightforward fashion to our setting.

3. Ruth type integrators: Our methods are, generally, implicit. But in particular examples they can be made explicit sometimes. That is very important in order to develop very efficient methods, a nice exposition of these topics is given in [27]. This is a classical fact, as it happens already in the symplectic case, classical references are [12, 31] where the authors develop fourth order explicit methods for mechanical Hamiltonians, that is, of the form kinetic plus potential energy. Our approach seems to be useful in that regard. For instance, consider the example introduced in 1 and assume that the moments of inertia of the rigid body are different, so the Hamiltonian reads,

$$
H\left(\Pi_{1}, \Pi_{2}, \Pi_{3}\right)=\frac{1}{2}\left(\frac{\Pi_{1}^{2}}{I^{1}}+\frac{\Pi_{2}^{2}}{I^{2}}+\frac{\Pi_{3}^{2}}{I^{3}}\right) .
$$

We can use the Casimir to simplify one of the terms without changing the dynamics,

$$
H^{\prime}=H-\frac{1}{2 I^{1}} C=\frac{1}{2}\left(\frac{I^{1}-I^{2}}{I^{1} I^{2}} \Pi_{2}^{2}+\frac{I^{1}-I^{3}}{I^{1} I^{3}} \Pi_{3}^{2}\right)=C^{1} \Pi_{2}^{2}+C^{2} \Pi_{3}^{2},
$$

where $C^{1}=\frac{I^{1}-I^{2}}{2 I^{1} I^{2}}$ and $C^{2}=\frac{I^{1}-I^{3}}{2 I^{1} I^{3}}$. And using the previous expression for $\tilde{\beta}$ we get

$$
H^{\prime} \circ \beta=C^{1}\left(\frac{\left[\left(p_{\varphi}-p_{\psi} \cos (\theta)\right) \cos (\psi)+p_{\theta} \sin (\theta) \sin (\psi)\right]^{2}}{I \sin ^{2}(\theta)}\right)+C^{2} p_{\psi}^{2}
$$

and the Hamilton-Jacobi equation becomes

$$
\frac{\partial S}{\partial t}+C^{1}\left(\frac{\left[\left(p_{\varphi}-p_{\psi} \cos \left(\frac{\partial S}{\partial p_{\theta}}\right)\right) \cos \left(\frac{\partial S}{\partial p_{\psi}}\right)+p_{\theta} \sin \left(\frac{\partial S}{\partial p_{\theta}}\right) \sin \left(\frac{\partial S}{\partial p_{\psi}}\right)\right]^{2}}{I \sin ^{2}\left(\frac{\partial S}{\partial p_{\theta}}\right)}\right)+C^{2} p_{\psi}^{2}=0
$$


This equation is not easily integrable, but clearly $H=H^{1}+H^{2}$ where

$$
\begin{aligned}
& H^{1}=C^{1}\left(\frac{\left[\left(p_{\varphi}-p_{\psi} \cos (\theta)\right) \cos (\psi)+p_{\theta} \sin (\theta) \sin (\psi)\right]^{2}}{I \sin ^{2}(\theta)}\right) \\
& H^{2}=C^{2} p_{\psi}^{2}
\end{aligned}
$$

and the Hamilton-Jacobi equation for each of the Hamiltonians is easily solvable by

$$
\begin{aligned}
& S^{1}\left(t, p_{\varphi}, p_{\theta}, p_{\psi}\right)=\frac{p_{\varphi}^{2}}{I^{\prime}} t+1 / 2 \pi p_{\psi}+1 / 2 \pi p_{\theta}+\pi p_{\varphi}, \\
& \text { and } \\
& S^{2}\left(t, p_{\varphi}, p_{\theta}, p_{\psi}\right) \quad=\frac{p_{\psi}^{2}}{I^{\prime}} t+\pi p_{\varphi}+1 / 2 \pi p_{\theta}+1 / 2 \pi p_{\psi},
\end{aligned}
$$

respectively. Each of the solutions gives and explicit transformation which is a rotation. The composition of the the explicit transformations induced by $S^{1}$ and $S^{2}$ gives the method introduced by R.I. McLachlan in [27], but we obtained it from the Hamilton-Jacobi theory. The development of a rigorous Ruth type integration techniques will provide very efficient numerical methods which conserve the geometry. We want to stress here that all the theoretical tools used in $[12,31]$ : the change of the Hamiltonian under a canonical transformation, the different types of generating functions,... were already introduced in this work. These results applied to the linear Poisson setting were not present in the literature until now, as far as we know. The importance of the groupoid setting was already noticed by C. Scovel and A.D. Weinstein. We recall the quote from [32], "The groupoid aspect of the theory also provides natural Poisson maps, useful in the application of Ruth type integration techniques, which do not seem easily derivable from the general theory of Poisson reduction".

4. Reduction of the Hamilton-Jacobi equation: Some of the authors of this paper developed a reduction and reconstruction procedure for the HamiltonJacobi equation, see [8], based on the following lemma.

Lemma 9. Let $(M, \Omega)$ a symplectic manifold, $G$ a connected Lie group and $\Phi: G \times M \rightarrow M$ an action by symplectomorphisms. Assume that this action has a equivariant momentum mapping, say $J: M \rightarrow \mathfrak{g}^{*}$. Given a (connected) Lagrangian submanifold $L \subset M$

(a) $L$ is a G-invariant Lagrangian submanifold,

(b) $J_{\mid L}=\mu$, i.e., $J$ is constant on $L$,

are equivalent.

The results introduced recently in [10] should permit the development of a reduction theory applicable to our framework. As an evidence of this fact, 
notice that in [10] the authors obtain a equivariant momentum mapping for an action which resembles the cotangent lifted actions. That setting is very similar to the one used by the authors in [8] and so it seems very likely to be that reduction theory applies to our theory.

5. Truncation of infinite-dimensional Poisson systems using linear Poisson structures: There are several examples of relevant physical importance which are infinite-dimensional Poisson systems: Euler equations of incompresible fluids, Vlasov-Maxwell, Vlasov-Poisson... Truncations of some of those systems conserving the geometry have already been carried out successfully, [27, 32, 34, 35]. In this regard, it seems that linear Poisson structures, i.e. dual bundles of Lie algebroids should be the natural setting for that. After that truncation is done, our methods could be applied in order to understand the qualitative behaviour of those infinite-dimensional systems.

6. Poincaré's generating function: Poincare's generating functions have been used in dynamical systems in order to relate critical points of a function to periodic orbits. Our setting admits an analogous theory using the coordinates introduced previously. We describe this statements in the Lie algebra case below.

Example 3. Let $G$ be a Lie group with Lie algebra $\mathfrak{g}$. Consider $\left(g^{i}, p_{i}\right)$ a set of coordinates introduced following Section 4.4.2. Then the following lemma holds.

Lemma 10. Let $S\left(p_{i}\right)$ a function such that the corresponding Lagrangian submanifold $\left\{\left(\frac{\partial S}{\partial p_{i}}, p_{i}\right)\right.$ such that $\left.p_{i} \in \mathbb{R}\right\}$ is a bisection, and let $\hat{S}: \mathfrak{g}^{*} \rightarrow$ $\mathfrak{g}^{*}$ be the induced Poisson transformation. Then the critical points of $S$ correspond to fixed points of $\hat{S}$.

Proof: The Lagrangian submanifold that generates the identity is given in those coordinates by

$$
L_{\text {id }}=\left\{\left(0^{i}, p_{i}\right) \text { such that } p_{i} \in \mathbb{R}\right\}
$$

and so $L_{\mathrm{id}} \cap \operatorname{graph}(d S)=\{$ critical points of $S\}$ which concludes the proof.

Similar results can be developed in the general situation.

\section{A Lie Algebroids}

In this section we introduce the definition of Lie algebroids. Associated to every Lie groupoid there is a Lie algebroid, as can be seen in Appendix B, although the converse is not true. 


\section{A.1 Definition}

A Lie algebroid is a vector bundle $\tau: A \rightarrow M$ endowed with the following data:

- A bundle map $\rho: A \rightarrow T M$ called the anchor.

- A Lie bracket on the space of sections $(\Gamma(\tau))$ satisfying the Leibniz identity, i.e.

$$
[[X, f Y]]=f[[X, Y]]+\mathcal{L}_{\rho(X)}(f) Y
$$

for all $X, Y \in \Gamma(\tau)$ and any $f \in C^{\infty}(M)$.

\section{A.2 Examples}

In this sections we introduce some examples of Lie algebroids. More examples, maybe the most natural ones, will be sketched in Appendix B when we talk about the Lie algebroids associated to a Lie groupoid.

\section{A.2.1 Vector Fields}

It can be seen that given a manifold $M$ there is a one-to-one correspondence between Lie algebroid structures on the trivial bundle $M \times \mathbb{R}$ and vector fields $X$ on $M$. The vector bundle $\tau: A=M \times \mathbb{R} \rightarrow M$ is given just by the projection onto $M$.

1. The anchor map $\rho: A \rightarrow T M$ is given by $\rho(m, t)=t X(m) \in T M$.

2. Given $f, g \in C^{\infty}(M)$ then $\left.\llbracket f f, g\right]=f X(g)-g X(f)$.

\section{A.2.2 2-forms}

Let $M$ be a manifold and consider now the bundle $A=T M \times \mathbb{R}$ over $M$, where the vector bundle structure $\tau: A \rightarrow M$ is the obvious one. Any closed 2-form $\omega$ on $M$ defines a Lie algebroid estructure on that bundle:

1. The anchor $\rho: A \rightarrow T M$ is given by the projection onto the first factor.

2. The Lie bracket is given by $[[(X, f),(Y, g)]=([X, Y], X(g)-Y(f)+$ $\omega(X, Y))$

Remark 25. This case can be used to find examples of non-integrable Lie algebroids.

\section{A.2.3 Poisson Manifolds}

One really important example of Lie algebroids is given by Poisson manifolds. Let $(P, \Lambda)$ be a Poisson manifold, then consider the bundle given by the cotangent bundle of $P, \pi_{P}: T^{*} P \rightarrow P$ and where the anchor and the Lie bracket are given by:

1. The anchor map is given the sharp of the Poisson tensor $\Lambda^{\sharp}: T^{*} P \rightarrow T P$. 
2. The Lie bracket is given by $[[\alpha, \beta]]=\mathcal{L}_{\Lambda^{\sharp}(\alpha)} \beta-\mathcal{L}_{\Lambda^{\sharp}(\beta)} \alpha-d(\Lambda(\alpha, \beta))$ for all $\alpha$ and $\beta$ 1-forms on $P$.

\section{A.3 The Poisson Structure of the Dual of a Lie Algebroid}

Given a Lie algebroid $\left(\tau: A \rightarrow M, \rho,[[\cdot, \cdot])\right.$, its dual $A^{*}$ has a natural linear Poisson structure that we proceed to describe now.

Given $X$ and $Y$ sections of $\tau$, then they determine linear functions on $A^{*}$ that we denote by $\hat{X}$ and $\hat{Y}$. On the other hand each $f \in C^{\infty}(M)$ determines a function which is constant on the fibers $f \circ \tau$. It can be seen that there exists a unique Poisson structure on $A^{*}$ which satisfies

$$
\begin{aligned}
& \{\hat{X}, \hat{Y}\}=\llbracket \hat{X, Y} \rrbracket \\
& \{\hat{X}, f \circ \tau\}=(\rho(X)(f)) \circ \tau \\
& \{f \circ \tau, g \circ \tau\}=0
\end{aligned}
$$

for all $X, Y \in \Gamma(\tau)$ and $f, g \in C^{\infty}(M)$.

Once we choose local basis of sections $e_{j} j=1, \ldots, m$ and local coordinates $x^{i} i=1, \ldots, n$ on $M$. This system induces local coordinates in $A^{*}$ by $\left(x^{i}, \mu_{i}\right)$, where $i=1, \ldots, n, j=1, \ldots, m$ and where $\mu_{i}=\hat{e}_{i}$. If the structure functions and the anchor map read locally

$$
\left[\left[e_{i}, e_{j}\right]\right]=c_{i j}^{k} e_{k} \quad \text { and } \quad \rho\left(e_{i}\right)=\rho_{i}^{j} \frac{\partial}{\partial x^{j}},
$$

then the Poisson bracket on the coordinates $\left(x^{i}, \mu_{j}\right)$ reads

$$
\begin{gathered}
\left\{x^{i}, x^{j}\right\}=0 \\
\left\{\mu^{i}, x^{j}\right\}=\rho_{i}^{j} \\
\left\{\mu^{i}, \mu^{j}\right\}=c_{i j}^{k} \mu_{k} .
\end{gathered}
$$

Due to our conventions, this Poisson structure is the opposite to the one that makes $\tilde{\beta}$ a Poisson mapping. So in our work we are considering the Poisson structure $\left(A^{*} G,-\{\cdot, \cdot\}\right)$.

\section{B Lie Groupoids}

We recall here the definition of a (Lie) groupoid. For more information about this concept, we refer the reader to the monograph [7] and K. Mackenzie's book [19].

\section{B.1 Definition}

Groupoids: A groupoid is a set $G$ equipped with the following data: 
1. another set $M$, called the base;

2. two surjective maps $\alpha: G \rightarrow M$ and $\beta: G \rightarrow M$, called, respectively, the source and target projections; we visualize an element $g \in G$ as an arrow from $\alpha(g)$ to $\beta(g)$ :

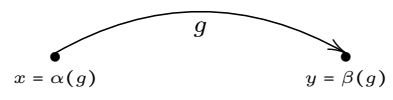

3. A partial multiplication, or composition map, $m: G_{2} \rightarrow G$ defined on the subset $G_{2}$ of $G \times G$ :

$$
G_{2}=\{(g, h) \in G \times G \mid \beta(g)=\alpha(h)\} .
$$

The multiplication will be denoted for simplicity by $m(g, h)=g h$. It verifies the following properties:

(a) $\alpha(g h)=\alpha(g)$ and $\beta(g h)=\beta(h)$.

(b) $(g h) k=g(h k)$.

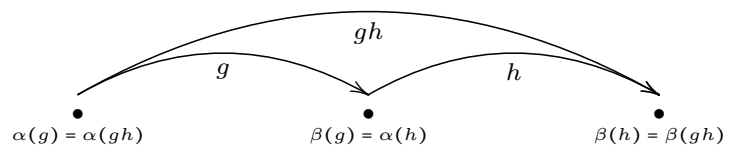

4. An identity section $\epsilon: M \rightarrow G$ such that

(a) $\epsilon(\alpha(g)) g=g$ and $g \epsilon(\beta(g))=g$ for all $g \in G$,

(b) $\alpha(\epsilon(x))=\beta(\epsilon(x))=x$ for all $x \in M$.

5. An inversion map $\iota: G \rightarrow G$, to be denoted simply by $\iota(g)=g^{-1}$, such that

(a) $g^{-1} g=\epsilon(\beta(g))$ and $g g^{-1}=\epsilon(\alpha(g))$.

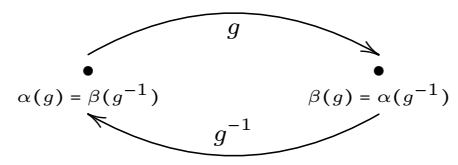

We will denote a groupoid $G$ over a base $M$ by $G \underset{\beta}{\stackrel{\alpha}{\rightrightarrows}} M$ or simply $G \rightrightarrows M$.

It is easy to see that $\epsilon$ must be injective, so there is a natural identification between $M$ and $\epsilon(M)$. However, we will keep a distinction between the two sets.

Lie Groupoids: A groupoid, $G \rightrightarrows M$, is said to be a Lie groupoid if $G$ and $M$ are differentiable manifolds, all the structural maps are differentiable and besides, $\alpha$ and $\beta$ differentiable submersions. If $G \rightrightarrows M$ is a Lie groupoid then $m$ is a submersion, $\epsilon$ is an embedding and $\iota$ is a diffeomorphism. Notice that since $\alpha$ and $\beta$ are submersions, the $\alpha$ and $\beta$-fibers are submanifolds. The same properties imply that $G_{2}$ is a submanifold. We will use $G^{x}=\alpha^{-1}(x), G_{y}=$ $\beta^{-1}(y)$ and $G_{y}^{x}=\alpha^{-1}(x) \cap \beta^{-1}(y)$. 
Left and Right multiplication: Given $g \in G_{y}^{x}$, so $g: x \rightarrow y$, we can define two (bijective) mappings $L_{g}: G^{y} \rightarrow G^{x}$ and and $R_{g}: G_{x} \rightarrow G_{y}$, which are the left translation by $g$ and the right translation by $g$ respectively. These diffeomorphisms are given by

$$
\begin{aligned}
L_{g}: \quad G^{y} & \longrightarrow G^{x} \\
h & \mapsto L_{g}(h)=g h \quad ; \quad R_{g}: \quad G_{x} \longrightarrow G_{y} \longrightarrow R_{g}(h)=h g .
\end{aligned}
$$

where we have that $\left(L_{g}\right)^{-1}=L_{g^{-1}}$ and $\left(R_{g}\right)^{-1}=R_{g^{-1}}$.

Invariant Vector Fields: A vector field $X$ on $G$ is said to be left-invariant (resp., right-invariant) if it is tangent to the fibers of $\alpha$ (resp., $\beta$ ) and

$$
X(g h)=\left(T_{h} L_{g}\right)(X(h)) \quad\left(\text { resp. } X(g h)=\left(T_{g} R_{h}\right)(X(g))\right)
$$

for all $(g, h) \in G_{2}$.

Morphisms: Given two Lie groupoids $G \rightrightarrows M$ and $G^{\prime} \rightrightarrows M^{\prime}$, a morphism of Lie groupoids is a smooth map $\Phi: G \rightarrow G^{\prime}$ such that

1. If $(g, h) \in G_{2}$ then $(\Phi(g), \Phi(h)) \in\left(G^{\prime}\right)_{2}$.

2. $\Phi(g h)=\Phi(g) \Phi(h)$.

A morphism of Lie groupoids $\Phi: G \rightarrow G^{\prime}$ induces a smooth map $\Phi_{0}: M \rightarrow M^{\prime}$ in such a way that the source and the target commute with the morphism, i.e.

$$
\alpha^{\prime} \circ \Phi=\Phi_{0} \circ \alpha, \quad \beta^{\prime} \circ \Phi=\Phi_{0} \circ \beta, \quad \Phi \circ \epsilon=\epsilon^{\prime} \circ \Phi_{0},
$$

$\alpha, \beta$ and $\epsilon$ (resp., $\alpha^{\prime}, \beta^{\prime}$ and $\epsilon^{\prime}$ ) being the source, the target and the identity sections of $G$ (resp., $\left.G^{\prime}\right)$.

\section{B.2 Lie Algebroid Associated to a Lie Groupoid}

Given a Lie groupoid $G$ we denote by $A G=\operatorname{ker}(T \alpha)_{\mid \epsilon(M)}$, i.e., the set of vectors tangent to the $\alpha$-fibers restricted to the units of the groupoid. Since the units, $\operatorname{Im}(\epsilon)$, are diffeomorphic to the base manifold, $M$, we will consider it as a vector bundle $\tau: A G \rightarrow M$. The reader should keep this identification in mind, because it is going to be used implicitly in some places $(M \equiv \operatorname{Im}(\epsilon) \subset G)$.

It is easy to prove that there exists a bijection between the space of sections $\Gamma(\tau)$ and the set of left-invariant (resp., right-invariant) vector fields on $G$. If $X$ is a section of $\tau: A G \rightarrow M$, the corresponding left-invariant (resp., rightinvariant) vector field on $G$ will be denoted $\overleftarrow{X}$ (resp., $\vec{X}$ ), where

$$
\begin{gathered}
\overleftarrow{X}(g)=\left(T_{\epsilon(\beta(g))} L_{g}\right)(X(\beta(g))), \\
\left.\vec{X}(g)=-\left(T_{\epsilon(\alpha(g))} R_{g} \circ \iota\right)(X(\alpha(g)))\right),
\end{gathered}
$$

for $g \in G$.

Using the above facts, we may introduce a Lie algebroid structure $([[\cdot, \cdot], \rho)$ on $A G$ : 
1. The anchor map $\rho: A G \rightarrow T M$ is

$$
\rho(X)(x)=\left(T_{\epsilon(x)} \beta\right)(X(x))
$$

for $X \in \Gamma(\tau)$ and $x \in M$.

2. The Lie bracket on the space of sections $\Gamma(\tau)$, denoted by $[[\cdot, \cdot]]$ is defined by

$$
\overleftarrow{[X, Y]}=[\overleftarrow{X}, \overleftarrow{Y}]
$$

for $X, Y \in \Gamma(\tau)$ and $x \in M$.

Note that

$$
\begin{gathered}
\overrightarrow{\llbracket X, Y \rrbracket}=-[\vec{X}, \vec{Y}], \quad[\vec{X}, \overleftarrow{Y}]=0, \\
T \iota \circ \vec{X}=-\overleftarrow{X} \circ \iota, \quad T \iota \circ \overleftarrow{X}=-\vec{X} \circ \iota
\end{gathered}
$$

(for more details, see [19]). The dual bundle of $A G$ will be denoted by $A^{*} G$.

In addition, define the vector bundle $V \alpha$ as the sub-bundle of $T G$ consisting of $\alpha$-vertical vectors, that is, vectors tangent to the $\alpha$-fibers. $V \beta$ is defined analogously. Thus $A G$ is the restriction of $V \alpha$ to $\epsilon(M)$.

\section{B.3 Examples of Lie groupoids}

Next, we will present some examples of Lie groupoids. The corresponding associated Lie algebroid is pointed out in each case.

\section{B.3.1 Lie Groups}

Any Lie group $G$ is a Lie groupoid over $\{e\}$, the identity element of $G$.

1. The source, $\alpha$, is the constant map $\alpha(g)=e$.

2. The target, $\beta$, is the constant $\operatorname{map} \beta(g)=e$.

3. The identity map is $\epsilon(e)=e$.

4. The inversion map is $\iota(g)=g^{-1}$.

5. The multiplication is $m(g, h)=g \cdot h$, for any $g$ and $h$ in $G$.

Associated Lie algebroid: The Lie algebroid associated with $G$ is just the Lie algebra $\mathfrak{g}$ of $G$ in a straightforward way. 


\section{B.3.2 The Pair or Banal Groupoid}

Let $M$ be a manifold. The product manifold $M \times M$ is a Lie groupoid over $M$ called the pair or banal groupoid. Its structure mappings are:

1. The source, $\alpha$, is the projection onto the first factor.

2. The target, $\beta$, is the projection onto the second factor.

3. The identity map is $\epsilon(x)=(x, x)$, for all $x \in M$.

4. The inversion map is $\iota(x, y)=(y, x)$.

5. The multiplication is $m((x, y),(y, z))=(x, z)$, for $(x, y),(y, z) \in M \times M$.

Associated Lie Algebroid: If $x$ is a point of $M$, it follows that

$$
A=\operatorname{ker}(T \alpha)_{\epsilon(x)}=\left\{0_{x}\right\} \times T_{x} M
$$

which gives the vector bundle structure and given $\left(0_{x}, X_{x}\right) \in A$, then

$$
\tau\left(0_{x}, X_{x}\right)=x .
$$

1. The anchor is given by the projection over the second factor $\rho\left(0_{x}, X_{x}\right)=$ $X_{x} \in T_{x} M$

2. The Lie bracket on the space of sections, $\Gamma(\tau)$, is the Lie bracket of vector field on the second factor $[(0, X),(0, Y)]=(0,[X, Y])$.

\section{B.3.3 Atiyah or Gauge Groupoids}

Let $\pi: P \rightarrow M$ be a principal $G$-bundle. Then the free action $\Phi: G \times P \rightarrow P$ induces the diagonal action $\Phi^{\prime}: G \times(P \times P) \rightarrow P \times P$ by $\Phi^{\prime}\left(g,\left(q, q^{\prime}\right)\right)=\left(g q, g q^{\prime}\right)$. Moreover, one may consider the quotient manifold $(P \times P) / G$ and it admits a Lie groupoid structure over $M$, called the Atiyah or Gauge groupoid (see, for instance, $[19,21])$. We describe now the structural mappings.

1. The source, $\alpha:(P \times P) / G \rightarrow M$ is given by $\left[\left(q, q^{\prime}\right)\right] \mapsto \pi(q)$.

2. The target, $\beta:(P \times P) / G \rightarrow M$ is given by $\left[\left(q, q^{\prime}\right)\right] \mapsto \pi\left(q^{\prime}\right)$.

3. The identity map, $\epsilon: M \rightarrow(P \times P) / G$ is $x \mapsto[(q, q)]$, if $\pi(q)=x$.

4. The inversion map, $\iota:(P \times P) / G \rightarrow(P \times P) / G$ is $\left[\left(q, q^{\prime}\right)\right] \mapsto\left[\left(q^{\prime}, q\right)\right]$.

5. The multiplication map $m:((P \times P) / G)_{2} \rightarrow(P \times P) / G$ is $\left(\left[\left(q, q^{\prime}\right)\right],\left[\left(g q^{\prime}, q^{\prime \prime}\right)\right]\right) \mapsto\left[\left(g q, q^{\prime \prime}\right)\right]$. 
Associated Lie Algebroid: It easily follows that $A=\operatorname{ker} T \alpha_{\epsilon(M)}$ can be identified with $T P / G$. Then the associated Lie algebroid is just $\tau: T P / G \rightarrow M$, where $\tau$ is the obvious projection. Then the Lie algebroid structure is provided by

1. The anchor, $\rho: T P / G \rightarrow M$, is given by the quotient of the natural projection map $\tau_{p}: T P \rightarrow P$. That is, $\rho=\tau_{P} / G: T P / G \rightarrow P / G=M$.

2. The Lie bracket on the space of sections is given by the Lie bracket of vector fields. It is easy to see that the Lie bracket of two $G$-invariant vector fields is another $G$-invariant vector field.

\section{B.3.4 Action Lie groupoids}

Let $G$ be a Lie group and let $\Phi: M \times G \rightarrow M,(x, g) \mapsto x g$, be a right action of $G$ on $M$. Consider the action Lie groupoid $M \times G$ over $M$ with structural maps given by

1. The source is $\alpha(x, g)=x$.

2. The target is $\beta(x, g)=x g$.

3. The identity map is $\epsilon(x)=(x, e)$.

4. The inversion map is $\iota(x, g)=\left(x g, g^{-1}\right)$.

5. The multiplication is $m\left((x, g),\left(x g, g^{\prime}\right)\right)=\left(x, g g^{\prime}\right)$

See, for instance, $[19,21]$ for the details.

Associated Lie Algebroid: Now, let $\mathfrak{g}=T_{e} G$ be the Lie algebra of $G$. Given $\xi \in \mathfrak{g}$ we will denote by $\xi_{M}$ the infinitesimal generator of the action $\Phi: M \times G \rightarrow M$. Consider now the vector bundle $\tau: M \times \mathfrak{g} \rightarrow M$ where $\tau$ is the projection over the first factor, endowed with the following structures:

1. The anchor is $\rho(x, \xi)=\xi_{M}(x)$.

2. The Lie bracket on the space of sections is given by $[[\widetilde{\xi}, \widetilde{\eta}](x)=[\widetilde{\xi}(x), \widetilde{\eta}(x)]+$ $(\widetilde{\xi}(x))_{M}(x)(\widetilde{\eta})-(\widetilde{\eta}(x))_{M}(x)(\widetilde{\xi})$ for $\widetilde{\xi}, \widetilde{\eta} \in \Gamma(\tau)$.

\section{Acknowledgments}

TThis work has been partially supported by MINECO MTM 2013-42 870-P, the European project IRSES-project "Geomech-246981" and the ICMAT Severo Ochoa project SEV-2011-0087. M. Vaquero wishes to thank MINECO for a FPI-PhD Position and David Iglesias-Ponte and Luis García-Naranjo for useful discussions. 


\section{References}

[1] Abraham, R., And Marsden, J. E. Foundations of mechanics. Benjamin/Cummings Publishing Co., Inc., Advanced Book Program, Reading, Mass., 1978. Second edition, revised and enlarged, With the assistance of Tudor Raţiu and Richard Cushman.

[2] Arnol'D, V. I. Mathematical methods of classical mechanics, vol. 60 of Graduate Texts in Mathematics. Springer-Verlag, New York, 1993. Translated from the 1974 Russian original by K. Vogtmann and A. Weinstein, Corrected reprint of the second (1989) edition.

[3] Benzel, S., Ge, Z., And Scovel, C. Elementary construction of higherorder Lie-Poisson integrators. Phys. Lett. A 174, 3 (1993), 229-232.

[4] Channell, P. J., And Scovel, C. Symplectic integration of Hamiltonian systems. Nonlinearity 3, 2 (1990), 231-259.

[5] Channell, P. J., And Scovel, J. C. Integrators for Lie-Poisson dynamical systems. Phys. D 50, 1 (1991), 80-88.

[6] Coste, A., Dazord, P., And Weinstein, A. Groupoïdes symplectiques. In Publications du Département de Mathématiques. Nouvelle Série. A, Vol. 2, vol. 87 of Publ. Dép. Math. Nouvelle Sér. A. Univ. Claude-Bernard, Lyon, 1987, pp. i-ii, 1-62.

[7] Crainic, M., and Fernandes, R. L. Lectures on integrability of Lie brackets. In Lectures on Poisson geometry, vol. 17 of Geom. Topol. Monogr. Geom. Topol. Publ., Coventry, 2011, pp. 1-107.

[8] de León, M., Martín de Diego, D., And Vaquero, M. HamitonJacobi theory, Symmetries and Coisotropic Reduction. Preprint, 2015.

[9] Feng, K., And QIn, M. Symplectic geometric algorithms for Hamiltonian systems. Zhejiang Science and Technology Publishing House, Hangzhou; Springer, Heidelberg, 2010. Translated and revised from the Chinese original, With a foreword by Feng Duan.

[10] Fernandes, R. L., Ortega, J.-P., and Ratiu, T. S. The momentum map in Poisson geometry. Amer. J. Math. 131, 5 (2009), 1261-1310.

[11] Ferraro, S., Jiménez, F., And Martín de Diego, D. New developments on the geometric nonholonomic integrator. Nonlinearity 28, 4 (2015), $871-900$.

[12] Forest, E., And Ruth, R. D. Fourth-order symplectic integration. Phys. $D$ 43, 1 (1990), 105-117.

[13] Ge, Z. Generating functions, Hamilton-Jacobi equations and symplectic groupoids on Poisson manifolds. Indiana Univ. Math. J. 39, 3 (1990), 859-876. 
[14] GE, Z. Equivariant symplectic difference schemes and generating functions. Phys. D 49, 3 (1991), 376-386.

[15] Ge, Z., and Marsden, J. E. Lie-Poisson Hamilton-Jacobi theory and Lie-Poisson integrators. Phys. Lett. A 133, 3 (1988), 134-139.

[16] Goldstein, H. Classical mechanics, second ed. Addison-Wesley Publishing Co., Reading, Mass., 1980. Addison-Wesley Series in Physics.

[17] Hairer, E., Lubich, C., And Wanner, G. Geometric numerical integration, vol. 31 of Springer Series in Computational Mathematics. Springer, Heidelberg, 2010. Structure-preserving algorithms for ordinary differential equations, Reprint of the second (2006) edition.

[18] Karasözen, B. Poisson integrators. Math. Comput. Modelling 40, 11-12 (2004), 1225-1244.

[19] MackenzIE, K. C. H. General theory of Lie groupoids and Lie algebroids, vol. 213 of London Mathematical Society Lecture Note Series. Cambridge University Press, Cambridge, 2005.

[20] Makazaga, J., And Murua, A. A new class of symplectic integration schemes based on generating functions. Numer. Math. 113, 4 (2009), 631642 .

[21] Marrero, J. C., Martín de Diego, D., And Martínez, E. Discrete Lagrangian and Hamiltonian mechanics on Lie groupoids. Nonlinearity 19, 6 (2006), 1313-1348.

[22] Marrero, J. C., Martín de Diego, D., And Martínez, E. On the exact discrete lagrangian function for variational integrators: Theory and applications. Preprint, 2015.

[23] Marrero, J. C., Martín de Diego, D., And Stern, A. Symplectic groupoids and discrete constrained Lagrangian mechanics. Discrete Contin. Dyn. Syst. 35, 1 (2015), 367-397.

[24] Marsden, J. E. Lectures on mechanics, vol. 174 of London Mathematical Society Lecture Note Series. Cambridge University Press, Cambridge, 1992.

[25] Marsden, J. E., Raţiu, T., and Weinstein, A. Semidirect products and reduction in mechanics. Trans. Amer. Math. Soc. 281, 1 (1984), 147177.

[26] Marsden, J. E., AND RATiU, T. S. Introduction to mechanics and symmetry, second ed., vol. 17 of Texts in Applied Mathematics. Springer-Verlag, New York, 1999. A basic exposition of classical mechanical systems.

[27] McLachlan, R. I. Explicit Lie-Poisson integration and the Euler equations. Phys. Rev. Lett. 71, 19 (1993), 3043-3046. 
[28] McLachlan, R. I., And Scovel, C. Equivariant constrained symplectic integration. J. Nonlinear Sci. 5, 3 (1995), 233-256.

[29] Mclachlan, R. I., And Scovel, C. A survey of open problems in symplectic integration. In Integration algorithms and classical mechanics (Toronto, ON, 1993), vol. 10 of Fields Inst. Commun. Amer. Math. Soc., Providence, RI, 1996, pp. 151-180.

[30] Moser, J., And Veselov, A. P. Discrete versions of some classical integrable systems and factorization of matrix polynomials. Comm. Math. Phys. 139, 2 (1991), 217-243.

[31] Ruth, R. D. A Canonical Integration Technique. IEEE Transactions on Nuclear Science 30 (Aug. 1983), 2669.

[32] Scovel, C., And Weinstein, A. Finite-dimensional Lie-Poisson approximations to Vlasov-Poisson equations. Comm. Pure Appl. Math. 47, 5 (1994), 683-709.

[33] Weinstein, A. Lagrangian mechanics and groupoids. In Mechanics day (Waterloo, ON, 1992), vol. 7 of Fields Inst. Commun. Amer. Math. Soc., Providence, RI, 1996, pp. 207-231.

[34] Zachos, C. K., And Fairlie, D. B. New infinite-dimensional algebras, sine brackets, and $\mathrm{SU}(\infty)$. In Strings '89 (College Station, TX, 1989). World Sci. Publ., River Edge, NJ, 1990, pp. 378-387.

[35] Zeituin, V. Finite-mode analogs of 2D ideal hydrodynamics: coadjoint orbits and local canonical structure. Phys. D 49, 3 (1991), 353-362. 


\title{
A HAMILTON-JACOBI THEORY ON POISSON MANIFOLDS
}

\author{
Manuel de León, David Martín de Diego and Miguel Vaquero \\ Instituto de Ciencias Matemáticas (CSIC-UAM-UC3M-UCM) \\ c\Nicolás Cabrera, n 13-15, Campus Cantoblanco, UAM \\ 28049 Madrid, Spain
}

(Communicated by Andrew Lewis)

\begin{abstract}
In this paper we develop a Hamilton-Jacobi theory in the setting of almost Poisson manifolds. The theory extends the classical Hamilton-Jacobi theory and can be also applied to very general situations including nonholonomic mechanical systems and time dependent systems with external forces.
\end{abstract}

1. Introduction. The standard formulation of the Hamilton-Jacobi problem is to find a function $S\left(t, q^{i}\right)$ (called the principal function) such that

$$
\frac{\partial S}{\partial t}+h\left(q^{i}, \frac{\partial S}{\partial q^{i}}\right)=0
$$

where $h=h\left(q^{i}, p_{i}\right)$ is the hamiltonian function of the system. If we put $S\left(t, q^{i}\right)=$ $W\left(q^{i}\right)-t E$, where $E$ is a constant, then $W$ satisfies

$$
h\left(q^{i}, \frac{\partial W}{\partial q^{i}}\right)=E
$$

$W$ is called the characteristic function.

Equations (1) and (2) are indistinctly referred as the Hamilton-Jacobi equation (see $[1,2,22])$.

The Hamilton-Jacobi equation helps to solve the Hamilton equations for $h$

$$
\frac{d q^{i}}{d t}=\frac{\partial h}{\partial p_{i}}, \frac{d p_{i}}{d t}=-\frac{\partial h}{\partial q^{i}} .
$$

Indeed, if we find a solution $W$ of the Hamilton-Jacobi equation (2) then any solution $\left(q^{i}(t)\right)$ of the first set of equations (3) gives a solution of the Hamilton equations by taking $p_{i}(t)=\frac{\partial W}{\partial q^{i}}$.

This result can be founded in [1]. Moreover, one can rephrase the above result by stating that if $W$ is a solution of the Hamilton-Jacobi equation, then $d W$ (a 1-form on $Q$ ) transforms the integral curves of the vector field $X_{h}^{d W}=T \pi_{Q} \circ X_{h} \circ d W$ into the integral curves of $X_{h}$; here, $X_{h}$ is the Hamiltonian vector field defined by the hamiltonian $h$ and $\pi_{Q}: T^{*} Q \longrightarrow Q$ is the canonical projection. Of course we can

2010 Mathematics Subject Classification. Primary: 70H20, 70 G45, 70F25, 37J60.

Key words and phrases. Hamilton-Jacobi theory, Poisson manifolds, nonholonomic mechanics, time-dependent systems, external forces.

This work has been partially supported by MICINN (Spain) MTM 2010-21186-C02-01, MTM 2009-08166-E, the European project IRSES-project "Geomech-246981" and the ICMAT Severo Ochoa project SEV-2011-0087. M. Vaquero wishes to thank MINECO for a FPI-PhD Position. The authors are also grateful to the referees for their useful comments. 
think in a more general situation where we look for general 1-forms on $Q$ that play a similar role to $d W$.

This geometrical procedure has been succesfully applied to many other different contexts, including nonholonomic mechanics (see $[7,8,11,13]$ ), singular lagrangian systems $[15,16]$, and even classical field theories [12, 17, 14]. Notice that in these frameworks, we don't have a symplectic framework; for instance, in nonholonomic mechanics the natural geometric framework is provided by a $(2,0)$-tensor field (an almost Poisson tensor) on the constraint submanifold that it is not integrable (that is, it is not satisfies the Jacobi identity). The almost-Poisson bracket in nonholonomic mechanics has been firstly introduced by A. van der Shaft and B.M. Mashke ([24]). All these scenarios are just the motivation for the investigation developed in this paper.

Our goal is to develop a Hamilton-Jacobi theory in a unifying and more general setting, say hamiltonian systems on an almost-Poisson manifold, that is, a manifold equipped with a skew-symmetric $(2,0)$-tensor field which does not necesarily satisfies the Jacobi identity. We also assume that the almost-Poisson manifold has a fibered structure over another manifold. The Hamilton-Jacobi problem now is to find a section of the fibered manifold such that its image is a lagrangian submanifold and the differential of the given hamiltonian vanishes on the tangent vectors to the section and belonging to the characteristic distribution.

The theory includes the case of classical hamiltonian systems on the cotangent bundle of the configuration manifold as well as the case of nonholonomic mechanical systems. We also apply the theory to time-dependent hamiltonian systems and systems with external forces.

We also discuss the existence of complete solutions and prove that if a complete solution exists then we obtain first integral in involution, which is a remarkable fact since our framework is just almost-Poisson.

Along the paper, all the manifolds are real, second countable and $\mathcal{C}^{\infty}$. The maps are assumed to be also $\mathcal{C}^{\infty}$. Sum over all repeated indices is understood

\section{Hamilton-Jacobi theory on almost-Poisson manifolds.}

2.1. Hamiltonian systems on almost-Poisson manifolds. Assume that $(E, \Lambda)$ is an almost-Poisson manifold, that is, $E$ is a manifold equipped with an almostPoisson structure $\Lambda$, which means that $\Lambda$ is a skew-symmetric $(2,0)$-tensor field on $E$. Notice that $\Lambda$ does not necessarily satisfy the Jacobi identy; in that case, we will have a Poisson tensor, and $E$ will be a Poisson manifold. For the moment, one only needs to ask $(E, \Lambda)$ be an almost-Poisson manifold.

Therefore, $\Lambda$ defines a vector bundle morphism

$$
\sharp: T^{*} E \longrightarrow T E
$$

by

$$
\langle\sharp(\alpha), \beta\rangle=\Lambda(\alpha, \beta)
$$

for all $\alpha, \beta \in T^{*} E$. Of course, we shall also denote by $\sharp$ the induced morphism of $\mathcal{C}^{\infty}$-modules between the spaces of 1 -forms and vector fields on $E$. Notice that we will use the notation $\sharp_{\Lambda}$ if there is danger of confusion.

We denote by $\mathcal{C}$ the characteristic distribution defined by $\Lambda$, that is

$$
\mathcal{C}_{p}=\sharp\left(T_{p}^{*} E\right)
$$


for all $p \in E$ (in other terms, $\mathcal{C}_{p}=\operatorname{Im} \sharp_{p}$, where $\sharp_{p}=T_{p}^{*} E \longrightarrow T_{p} E$ ). The rank of the almost-Poisson structure at $p$ is the dimension of the space $\mathcal{C}_{p}$. Notice that $\mathcal{C}$ is a generalized distribution and, moreover, is not (in general) integrable since $\Lambda$ is not Poisson in principle.

The following lemma will be useful

Lemma 2.1. Let $(E, \Lambda)$ be an almost-Poisson manifold, then we have

$$
\mathcal{C}^{\circ}=\operatorname{ker}(\sharp),
$$

where $\mathcal{C}^{\circ}$ denotes the annihilator of $\mathcal{C}$.

Proof. Observe that

$$
\begin{aligned}
\left(\operatorname{Im} \sharp_{p}\right)^{\circ} & =\left\{\mu \in T_{p}^{*} E \mid\left\langle\mu, \sharp_{p}(\alpha)\right\rangle=0, \forall \alpha \in T_{p}^{*} E\right\} \\
& =\left\{\mu \in T_{p}^{*} E \mid\left\langle\sharp_{p}(\mu), \alpha\right\rangle=0, \forall \alpha \in T_{p}^{*} E\right\} \\
& =\operatorname{ker} \sharp_{p}
\end{aligned}
$$

and thus, the result holds.

We also have the following definition

Definition 2.2. ([19, 23]) A submanifold $N$ of $E$ is said to be a lagrangian submanifold if the following equality holds

$$
\sharp\left(T N^{\circ}\right)=T N \cap \mathcal{C} .
$$

To have dynamics we need to introduce a hamiltonian function $h: E \longrightarrow \mathbb{R}$, and thus we obtain the corresponding hamiltonian vector field

$$
X_{h}=\sharp(d h) .
$$

2.2. Hamilton-Jacobi theory on almost-Poisson manifolds. Assume now that the almost-Poisson manifold $E$ with almost-Poisson tensor $\Lambda$ fibres over a manifold $M$, say $\pi: E \longrightarrow M$ is a surjective submersion (in other words, a fibration).

Assume that $\gamma$ is a section of $\pi: E \longrightarrow M$, i.e. $\pi \circ \gamma=i d_{M}$. Define the vector field $X_{h}^{\gamma}$ on $M$ by

$$
X_{h}^{\gamma}=T \pi \circ X_{h} \circ \gamma
$$

The following diagram summarizes the above construction:

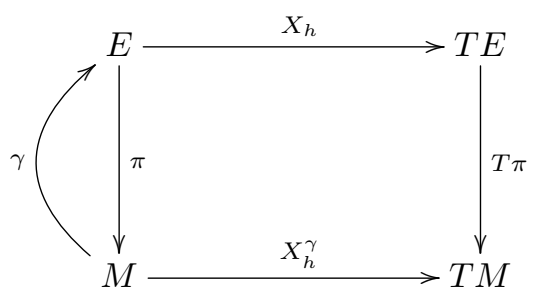

The following result relates the integral curves of $X_{h}$ and $X_{h}^{\gamma}$.

Theorem 2.3. Assume that $\operatorname{Im}(\gamma)$ is a lagrangian submanifold of $(E, \Lambda)$. Then the following assertions are equivalent:

1. $X_{h}$ and $X_{h}^{\gamma}$ are $\gamma$-related, i.e. $T \gamma \circ X_{h}^{\gamma}=X_{h} \circ \gamma$,

2. $d h \in(T \operatorname{Im}(\gamma) \cap \mathcal{C})^{\circ}$. 
Proof. " (i) $\Rightarrow$ (ii)"

Assume that $X_{h}$ and $X_{h}^{\gamma}$ are $\gamma$-related. Then $X_{h}=T \gamma\left(X_{h}^{\gamma}\right)$ and since $X_{h} \in \mathcal{C}$, we have $X_{h} \in T \operatorname{Im}(\gamma) \cap \mathcal{C}$. But $\operatorname{Im}(\gamma)$ is a lagrangian submanifold, so there exists $\beta \in(T \operatorname{Im}(\gamma))^{\circ}$ such that

$$
X_{h}=\sharp(\beta),
$$

along the image of $\gamma$.

Using that $X_{h}=\sharp(d h)$, we have $\sharp(d h-\beta)=0$, so $d h-\beta \in \operatorname{Ker} \sharp=\mathcal{C}^{\circ}$.

Therefore

$$
\begin{gathered}
d h \in \beta+\mathcal{C}^{\circ} \subset(T \operatorname{Im}(\gamma))^{\circ}+\mathcal{C}^{\circ} \\
=(T \operatorname{Im}(\gamma) \cap \mathcal{C})^{\circ} .
\end{gathered}
$$

"(ii) $\Leftarrow(\mathrm{i})$ "

If $d h \in(T \operatorname{Im}(\gamma) \cap \mathcal{C})^{\circ}=T \operatorname{Im}(\gamma)^{\circ}+\mathcal{C}^{\circ}$, then $d h=\alpha_{1}+\alpha_{2}$ where $\alpha_{1} \in T \operatorname{Im}(\gamma)^{\circ}$ and $\alpha_{2} \in \mathcal{C}^{\circ}$.

Then, along $\operatorname{Im}(\gamma)$ we have

$$
X_{h}=X_{\alpha_{1}}+X_{\alpha_{2}},
$$

where $\sharp\left(\alpha_{i}\right)=X_{\alpha_{i}}, i=1,2$. Using Lemma 2.1 we get $X_{h}=X_{\alpha_{1}}+X_{\alpha_{2}}=X_{\alpha_{1}}+0=$ $X_{\alpha_{1}}$, where $\alpha_{1} \in T \operatorname{Im}(\gamma)^{\circ}$.

Since $\operatorname{Im}(\gamma)$ is a lagrangian submanifold, we have

$$
\sharp\left(T \operatorname{Im}(\gamma)^{\circ}\right)=T \operatorname{Im}(\gamma) \cap \mathcal{C}
$$

and then

$$
X_{h}=X_{\alpha_{1}} \in T \operatorname{Im}(\gamma) \cap \mathcal{C} .
$$

Therefore we deduce that $X_{h}$ and $X_{h}^{\gamma}$ are $\gamma$-related since both are tangent to the submanifold $\operatorname{Im} \gamma$.

Assume that $(E, \Lambda)$ is a transitive Poisson manifold (a symplectic manifold), that is, $\mathcal{C}=T E$. Then, we have that a submanifold $N$ of a transitive Poisson manifold $(E, \Lambda)$ is a lagrangian submanifold if and only if

$$
\sharp\left(T N^{\circ}\right)=T N \text {. }
$$

Therefore, the above Theorem 2.3 takes the following classical form.

Theorem 2.4. Assume that $\operatorname{Im}(\gamma)$ is a lagrangian submanifold of a transitive Poisson manifold $(E, \Lambda)$. Then the following assertions are equivalent:

1. $X_{h}$ and $X_{h}^{\gamma}$ are $\gamma$-related;

2. $d(h \circ \gamma)=0$.

3. Computations in local coordinates. Assume that $\left(x^{i}, y^{a}\right)$ are local coordinates adapted to the fibration $\pi: E \longrightarrow M$, that is, $\pi\left(x^{i}, y^{a}\right)=\left(x^{i}\right)$, where $\left(x^{i}\right)$ are local coordinates in $M$.

Therefore, the tensor $\Lambda$ can be locally expressed as follows

$$
\Lambda=\frac{1}{2} \Lambda^{i j} \frac{\partial}{\partial x^{i}} \wedge \frac{\partial}{\partial x^{j}}+\Lambda^{i b} \frac{\partial}{\partial x^{i}} \wedge \frac{\partial}{\partial y^{b}}+\frac{1}{2} \Lambda^{a b} \frac{\partial}{\partial y^{a}} \wedge \frac{\partial}{\partial y^{b}},
$$

where $\Lambda_{i j}=-\Lambda_{j i}, \Lambda_{a b}=-\Lambda_{b a}$ due to the antisymmetry of $\Lambda$. Observe that

$$
\begin{aligned}
& \Lambda^{i j}=\Lambda\left(d x^{i}, d x^{j}\right), \Lambda^{i b}=\Lambda\left(d x^{i}, d y^{b}\right), \\
& -\Lambda^{j a}=\Lambda\left(d y^{a}, d x^{j}\right), \Lambda^{a b}=\Lambda\left(d y^{a}, d y^{b}\right) .
\end{aligned}
$$


The above local expressions implies that

$$
\begin{aligned}
& \sharp\left(d x^{i}\right)=\Lambda^{i j} \frac{\partial}{\partial x^{j}}+\Lambda^{i b} \frac{\partial}{\partial y^{b}} \\
& \sharp\left(d y^{a}\right)=-\Lambda^{j a} \frac{\partial}{\partial x^{j}}+\Lambda^{a b} \frac{\partial}{\partial y^{b}} .
\end{aligned}
$$

Using (4) we deduce that a hamiltonian vector field $X_{h}$ for a hamiltonian function $h \in C^{\infty}(E)$ is locally expressed by

$$
\begin{aligned}
X_{h}= & \left(\frac{\partial h}{\partial x^{i}} \Lambda^{i j}-\frac{\partial h}{\partial y^{a}} \Lambda^{j a}\right) \frac{\partial}{\partial x^{j}} \\
& +\left(\frac{\partial h}{\partial x^{i}} \Lambda^{i b}+\frac{\partial h}{\partial y^{a}} \Lambda^{a b}\right) \frac{\partial}{\partial y^{b}} .
\end{aligned}
$$

Now, let $\gamma: M \longrightarrow E$ be a section of $\pi: E \longrightarrow M$. If

$$
\gamma\left(x^{i}\right)=\left(x^{i}, \gamma^{a}\left(x^{i}\right)\right)
$$

we obtain

$$
X_{h}^{\gamma}=\left(\frac{\partial h}{\partial x^{i}} \Lambda^{i j}-\frac{\partial h}{\partial y^{a}} \Lambda^{j a}\right) \circ \gamma \frac{\partial}{\partial x^{j}} .
$$

Proposition 1. $\operatorname{Im}(\gamma)$ is a lagrangian submanifold of $(E, \Lambda)$ if and only if

$$
\Lambda^{a b}-\Lambda^{j b} \frac{\partial \gamma^{a}}{\partial x^{j}}+\Lambda^{j a} \frac{\partial \gamma^{b}}{\partial x^{j}}+\Lambda^{i j} \frac{\partial \gamma^{a}}{\partial x^{i}} \frac{\partial \gamma^{b}}{\partial x^{j}}=0
$$

Proof. First of all, let us observe that $T \operatorname{Im}(\gamma)$ is locally generated by the local vector fields

since

$$
\left\{\frac{\partial}{\partial x^{i}}+\frac{\partial \gamma^{a}}{\partial x^{i}} \frac{\partial}{\partial y^{a}}\right\}
$$

$$
T \gamma\left(\frac{\partial}{\partial x^{i}}\right)=\frac{\partial}{\partial x^{i}}+\frac{\partial \gamma^{a}}{\partial x^{i}} \frac{\partial}{\partial y^{a}}
$$

Therefore, if a 1-form on $E$

$$
\alpha=\alpha_{i} d x^{i}+\alpha_{a} d y^{a}
$$

annihilates $T \operatorname{Im}(\gamma)$ (recall that this is only valid along the points of $\operatorname{Im}(\gamma)$ ) we deduce the following conditions on the coefficients:

$$
\alpha_{i}=-\alpha_{a} \frac{\partial \gamma^{a}}{\partial x^{i}} .
$$

Now, a simple computation shows that

$$
\sharp(\alpha)=\left(\alpha_{i} \Lambda^{i j}-\alpha_{a} \Lambda^{j a}\right) \frac{\partial}{\partial x^{j}}+\left(\alpha_{i} \Lambda^{i b}+\alpha_{a} \Lambda^{a b}\right) \frac{\partial}{\partial y^{b}} .
$$

Assume now that $\operatorname{Im}(\gamma)$ is a lagrangian submanifold. Then, $\sharp(\alpha) \in T \operatorname{Im}(\gamma)$, with $\alpha \in T \gamma(M)^{\circ}$, and using (10) we deduce that

$$
\begin{aligned}
\sharp(\alpha) & =\left(\alpha_{i} \Lambda^{i j}-\alpha_{a} \Lambda^{j a}\right) \frac{\partial}{\partial x^{j}}+\left(\alpha_{i} \Lambda^{i b}+\alpha_{a} \Lambda^{a b}\right) \frac{\partial}{\partial y^{b}} \\
& =\alpha_{a}\left(-\frac{\partial \gamma^{a}}{\partial x^{i}} \Lambda^{i j}-\Lambda^{j a}\right) \frac{\partial}{\partial x^{i}}+\alpha_{a}\left(-\frac{\partial \gamma^{a}}{\partial x^{i}} \Lambda^{i b}+\Lambda^{a b}\right) \frac{\partial}{\partial y^{b}} \\
& =\lambda^{j}\left(\frac{\partial}{\partial x^{j}}+\frac{\partial \gamma^{b}}{\partial x^{j}} \frac{\partial}{\partial y^{b}}\right)
\end{aligned}
$$


which implies

and

$$
\lambda^{j}=-\alpha_{a} \frac{\partial \gamma^{a}}{\partial x^{i}} \Lambda^{i j}-\alpha_{a} \Lambda^{j a}
$$

$$
\lambda^{j} \frac{\partial \gamma^{b}}{\partial x^{j}}=-\alpha_{a} \frac{\partial \gamma^{a}}{\partial x^{i}} \Lambda^{i b}+\alpha_{a} \Lambda^{a b} .
$$

Substituting the values of $\lambda^{j}$ given by (11) in equation (12) we obtain

$$
\Lambda^{a b}-\Lambda^{j b} \frac{\partial \gamma^{a}}{\partial x^{j}}+\Lambda^{j a} \frac{\partial \gamma^{b}}{\partial x^{j}}+\Lambda^{i j} \frac{\partial \gamma^{a}}{\partial x^{i}} \frac{\partial \gamma^{b}}{\partial x^{j}}=0 .
$$

Assume now that equations (9) hold. Then, given an element $\alpha \in(T \operatorname{Im}(\gamma))^{\circ}$

$$
\begin{aligned}
\sharp(\alpha) & =\alpha_{a}\left(-\frac{\partial \gamma^{a}}{\partial x^{i}} \Lambda^{i j}-\Lambda^{j a}\right) \frac{\partial}{\partial x^{i}}+\alpha_{a}\left(-\frac{\partial \gamma^{a}}{\partial x^{i}} \Lambda^{i b}+\Lambda^{a b}\right) \frac{\partial}{\partial y^{b}} \\
& =\left(\alpha_{a}\left(-\Lambda^{i j} \frac{\partial \gamma^{a}}{\partial x^{i}}-\Lambda^{j a}\right)\right)\left(\frac{\partial}{\partial x^{i}}+\frac{\partial \gamma^{b}}{\partial x^{i}} \frac{\partial}{\partial y^{b}}\right)
\end{aligned}
$$

and therefore

$$
\sharp\left(T \operatorname{Im}(\gamma)^{\circ}\right)=T \operatorname{Im}(\gamma) \cap \mathcal{C} .
$$

4. Complete solutions. The essential idea in the standard Hamilton-Jacobi theory consists in finding a complete family of solutions to the problem (not only one particular solution). Therefore, in this section we shall discuss the notion of complete solutions for the Hamilton-Jacobi equation in this general framework of almost-Poisson manifolds. As a consequence we recover in a really simple and geometric way the results about complete solutions proved in [7].

First of all, we shall introduce the notion of complete solution.

Assume that we have a hamiltonian system given by a hamiltonian function $h: E \longrightarrow \mathbb{R}$ on an almost Poisson manifold $(E, \Lambda)$ fibered over a base manifold $M$. We assume that $\operatorname{dim} M=n$ and $\operatorname{dim} E=n+k$.

Definition 4.1. Consider $U \subset \mathbb{R}^{k}$ an open set, where $k$ is the dimension of the fiber of the bundle $\pi: E \rightarrow M$. A map $\Phi: M \times \mathbb{R}^{k} \rightarrow E$ is a complete solution if

1. $\Phi$ is a local diffeomorphism.

2. For any $\lambda=\left(\lambda_{1}, \ldots, \lambda_{k}\right) \in \mathbb{R}^{k}$, the map

$$
\begin{aligned}
\Phi_{\lambda}: M & \longrightarrow E \\
x & \rightarrow \Phi_{\lambda}(x)=\Phi\left(x, \lambda_{1} \ldots, \lambda_{k}\right)
\end{aligned}
$$

is a solution of the Hamilton-Jacobi problem for $h$.

For the sake of simplicity we will assume that $\Phi$ is a global diffeomorphism. Consider $f_{a}: E \rightarrow \mathbb{R}$ given the composition of $\Phi^{-1}$ with the projection over the $a$-th component of $\mathbb{R}^{k}$. We obtain

Proposition 2. The functions $f_{a}, 1 \leq a \leq k$ are in involution, that is, $\left\{f_{a}, f_{b}\right\}=0$, for all $a, b$, where $\{\cdot, \cdot\}$ is the bracket determined by $\Lambda$.

Proof. Given $p \in E$ we will show that $\left\{f_{a}, f_{b}\right\}(p)=0$. Suppose that $f_{a}(p)=\lambda_{a}$, for each $a=1, \ldots, k$, and observe that $p \in \operatorname{Im}\left(\Phi_{\lambda}\right)=\cap_{a} f_{a}^{-1}\left(\lambda_{a}\right)$. Therefore, we deduce that

$$
d f_{a}(p)_{\mid T \operatorname{Im}\left(\Phi_{\lambda}\right)}=0
$$


since $f_{a} \circ \Phi_{\lambda}$ is a constant function. By the hypothesis, $\operatorname{Im}\left(\Phi_{\lambda}\right)$ is a lagrangian submanifold in the sense previously explained, so

$$
\sharp\left(T \operatorname{Im}\left(\Phi_{\lambda}\right)^{\circ}\right)=T \operatorname{Im}\left(\Phi_{\lambda}\right) \cap \mathcal{C}
$$

and then

$$
X_{f_{a}}(p)=\sharp_{p}\left(d f_{a}(p)\right) \in T \operatorname{Im}\left(\Phi_{\lambda}\right) \cap \mathcal{C} .
$$

The result now follows since

$$
\left\{f_{a}, f_{b}\right\}(p)=d f_{a}(p)\left(X_{f_{b}}(b)\right)
$$

and the fact that $d f_{a} \in\left(T \operatorname{Im}\left(\Phi_{\lambda}\right) \cap \mathcal{C}\right)^{\circ}$ and $X_{f_{b}} \in T \operatorname{Im}\left(\Phi_{\lambda}\right) \cap \mathcal{C}$.

\section{Applications.}

5.1. Classical hamiltonian systems. (see $[1,2,18]$ )

A classical hamiltonian system is given by a hamiltonian function $h$ defined on the cotangent bundle $T^{*} Q$ of the configuration manifold $Q$.

If it is the case, then $E=T^{*} Q$ and $\Lambda$ is the canonical Poisson structure $\Lambda_{Q}$ on $T^{*} Q$ provided by the canonical symplectic form $\omega_{Q}$ on $T^{*} Q$. Recall that now we can take canonical bundle coordinates $\left(q^{i}, p_{i}\right)$, where $\pi_{Q}\left(q^{i}, p_{i}\right)=\left(q^{i}\right)$, and $\pi_{Q}: T^{*} Q \longrightarrow Q$ is the canonical projection.

Since in bundle coordinates

$$
\omega_{Q}=d q^{i} \wedge d p_{i}
$$

then

Therefore,

$$
\Lambda_{Q}=\frac{\partial}{\partial p_{i}} \wedge \frac{\partial}{\partial q^{i}}
$$

$$
X_{h}=\frac{\partial h}{\partial p_{i}} \frac{\partial}{\partial q^{i}}-\frac{\partial h}{\partial q^{i}} \frac{\partial}{\partial p_{i}}
$$

and if a section $\gamma: Q \longrightarrow T^{*} Q$ (that is, a 1-form on $Q$ ) is locally expressed by

$$
\gamma\left(q^{i}\right)=\left(q^{i}, \gamma_{i}(q)\right)
$$

we obtain

$$
X_{h}^{\gamma}=\left(\frac{\partial h}{\partial p_{i}} \circ \gamma\right) \frac{\partial}{\partial q^{i}} .
$$

The notion of lagrangian submanifold defined in Section 2 in the almost-Poisson setting reduces to the well-known in the symplectic setting, that is, it is isotropic and coisotropic with respect to the symplectic form $\omega_{Q}$.

If we compute the condition (9) for the current case we obtain

$$
\frac{\partial \gamma_{i}}{\partial q^{j}}=\frac{\partial \gamma_{j}}{\partial q^{i}}
$$

which just means that $\gamma$ is a closed form, i.e., $d \gamma=0$. So we recover the classical result (see $[1,2])$.

Proposition 3. Given a 1-form $\gamma$ on $Q$, we have that $\operatorname{Im}(\gamma)$ is a lagrangian submanifold of $\left(T^{*} Q, \Lambda_{Q}\right)$ if and only if $\gamma$ is closed.

As a consequence, we deduce the classical result directly from Theorem 2.4:

Theorem 5.1. Let $\gamma$ be a closed 1-form on $Q$. Then the following assertions are equivalent:

1. $X_{h}$ and $X_{h}^{\gamma}$ are $\gamma$-related; 
2. $d(h \circ \gamma)=0$.

5.2. Nonholonomic mechanical systems. In this section we will recover the results obtained in two previous papers [11, 13] (see also [8, 10, 21]).

A nonholonomic mechanical system is given by a lagrangian function $L: T Q \longrightarrow$ $\mathbb{R}$ subject to contraints determined by a linear distribution $D$ on the configuration manifold $Q$. We will denote by $\mathcal{D}$ the total space of the corresponding vector sub-bundle $\left(\tau_{Q}\right)_{\mid \mathcal{D}}: \mathcal{D} \longrightarrow Q$ defined by $D$, where $\left(\tau_{Q}\right)_{\mid \mathcal{D}}$ is the restriction of the canonical projection $\tau_{Q}: T Q \longrightarrow Q$.

We will assume that the lagrangian $L$ is defined by a Riemannian metric $g$ on $Q$ and a potential energy $V \in C^{\infty}(Q)$, so that

$$
L\left(v_{q}\right)=\frac{1}{2} g\left(v_{q}, v_{q}\right)-V(q)
$$

or, in bundle coordinates $\left(q^{i}, \dot{q}^{i}\right)$

$$
L\left(q^{i}, \dot{q}^{i}\right)=\frac{1}{2} g_{i j} \dot{q}^{i} \dot{q}^{j}-V\left(q^{i}\right)
$$

If $\left\{\mu^{a}\right\}, 1 \leq a \leq k$ is a local basis of the annihilator $D^{o}$ of $D$, then the constraints are locally expressed as

$$
\mu_{i}^{a}(q) \dot{q}^{i}=0
$$

where $\mu^{a}=\mu_{i}^{a}(q) d q^{i}$

The nonholonomic equations can be written as

$$
\begin{aligned}
& \frac{d}{d t}\left(\frac{\partial L}{\partial \dot{q}^{i}}\right)-\frac{\partial L}{\partial q^{i}}=\lambda^{i} \mu_{i}^{a}(q) \\
& \mu_{i}^{a}(q) \dot{q}^{i}=0,
\end{aligned}
$$

for some Lagrange multipliers $\lambda^{i}$ to be determined.

Let $S$ (respectively, $\Delta$ ) be the canonical vertical endomorphism (respectively, the Liouville vector field) on $T Q$. In local coordinates, we have

$$
S=d q^{i} \otimes \frac{\partial}{\partial \dot{q}^{i}}, \Delta=\dot{q}^{i} \frac{\partial}{\partial \dot{q}^{i}} .
$$

Therefore, we can construct the Poincaré-Cartan 2-form $\omega_{L}$ $=-d S^{*}(d L)$ and the energy function function $E_{L}=\Delta(L)-L$, such that the equation

$$
i_{\xi_{L}} \omega_{L}=d E_{L}
$$

has a unique solution, $\xi_{L}$, which is a SODE on $T Q$ (that is, $S\left(\xi_{L}\right)=\Delta$ ). Furthermore, its solutions coincide with the solutions of the Euler-Lagrange equations for $L$ :

$$
\frac{d}{d t}\left(\frac{\partial L}{\partial \dot{q}^{i}}\right)-\frac{\partial L}{\partial q^{i}}=0
$$

If we modify (13) as follows:

$$
\begin{array}{r}
i_{X} \omega_{L}-d E_{L} \in S^{*}\left((T \mathcal{D})^{o}\right) \\
X \in T \mathcal{D}
\end{array}
$$

the unique solution $X_{n h}$ is again a SODE whose solutions are just the ones of the nonholonomic equations.

Let

$$
F L: T Q \longrightarrow T^{*} Q
$$


be the Legendre transformation given by

$$
F L\left(q^{i}, \dot{q}^{i}\right)=\left(q^{i}, p_{i}=\frac{\partial L}{\partial \dot{q}^{i}}=g_{i j} \dot{q}^{j}\right)
$$

$F L$ is a global diffeomorphism which permits to reinterpret the nonholonomic mechanical system in the hamiltonian side. Indeed, we denote by $h=E_{L} \circ F L^{-1}$ the hamiltonian function and by $M=F L(\mathcal{D})$ the constraint submanifold of $T^{*} Q$.

The nonholonomic equations are then given by

$$
\begin{aligned}
\frac{d q^{i}}{d t} & =\frac{\partial h}{\partial p_{i}} \\
\frac{d p_{i}}{d t} & =-\frac{\partial h}{\partial q^{i}}+\bar{\lambda}^{i} \mu_{i}^{a},
\end{aligned}
$$

where $\bar{\lambda}^{i}$ are new Lagrange multipliers to be determined.

As above, the symplectic equation

$$
i_{X_{h}} \omega_{Q}=d h
$$

which gives the hamiltonian vector field $X_{h}$ should be modified as follows to take into account the nonholonomic constraints:

$$
\begin{array}{r}
i_{X} \omega_{Q}-d h \in F^{o} \\
X \in T M
\end{array}
$$

where $F$ is a distribution along $M$ whose annihilator $F^{o}$ is obtained from $S^{*}\left((T \mathcal{D})^{o}\right)$ through $F L$. Equations (16) and (17) have a unique solution, the nonholonomic vector field $\bar{X}_{n h}$.

An alternative way to obtain $\bar{X}_{n h}$ is to consider the Whitney sum decomposition

$$
T\left(T^{*} Q\right)_{\mid M}=T M \oplus F^{\perp}
$$

where the complement is taken with respect to $\omega_{Q}$. If

$$
P: T\left(T^{*} Q\right)_{\mid M} \longrightarrow T M
$$

is the canonical projection onto the first factor, one easily proves that

$$
\bar{X}_{n h}=P\left(X_{h}\right)
$$

Moreover, one can introduce an almost-Poisson tensor $\Lambda_{n h}$ on $M$ by

$$
\Lambda_{n h}(\alpha, \beta)=\Lambda_{Q}\left(P^{*} \alpha, P^{*} \beta\right) ;
$$

its associated bracket is called the nonholonomic bracket. Let us recall that this bracket has been firstly introduced and studied by A. van der Shaft and B.M. Mashke ([24]).

Obviously, we have

where $\sharp_{n h}$ stands for $\sharp_{\Lambda_{n h}}$.

$$
\bar{X}_{n h}=\sharp_{n h}(d h),
$$

An alternative way to define the nonholonomic bracket is as follows. Consider the distribution

$$
T M \cap F
$$

along $M$. A direct computation shows that the subspace

$$
T_{p} M \cap F_{p}
$$

is symplectic within the symplectic vector space $\left(T_{p}\left(T^{*} Q\right), \omega_{Q}(p)\right)$, for every $p \in M$ $($ see $[4,6])$. 
Thus we have a second Whitney sum decomposition

$$
T\left(T^{*} Q\right)_{\mid M}=(T M \cap F) \oplus(T M \cap F)^{\perp}
$$

where the complement is taken with respect to $\omega_{Q}$.

If

$$
\tilde{P}: T\left(T^{*} Q\right)_{\mid M} \longrightarrow T M \cap F
$$

is the canonical projection onto the first factor, one easily proves that

$$
\bar{X}_{n h}=\tilde{P}\left(X_{h}\right) \text {. }
$$

Moreover, it is possible to write $\Lambda_{n h}$ in terms of the projection $\tilde{P}$ as follows the the nonholonomic almost-Poisson tensor $\Lambda_{n h}$ on $M$ is now rewritten as

$$
\Lambda_{n h}(\alpha, \beta)=\Lambda_{Q}\left(\tilde{P}^{*} \alpha, \tilde{P}^{*} \beta\right)=\omega_{Q}\left(\tilde{P}\left(X_{\alpha}\right), \tilde{P}\left(X_{\beta}\right)\right)
$$

(see [6] for a proof).

Consider now the fibration

$$
\begin{gathered}
\left(M, \Lambda_{n h}\right) \\
\downarrow^{\pi_{Q_{\mid M}}} \\
Q
\end{gathered}
$$

and the hamiltonian $h_{\mid M}$ (also denoted by $h$ for sake of simplicity).

We can easily prove that

$$
\mathcal{C}_{p}=T_{p} M \cap F_{p}
$$

Indeed,we have

$$
\begin{aligned}
\left\langle\sharp_{n h}(\alpha), \beta\right\rangle & =-\omega_{Q}\left(\tilde{P} X_{\alpha}, X_{\beta}\right)=\omega_{Q}\left(X_{\beta}, \tilde{P} X_{\alpha}\right) \\
& =\left(i_{X_{\beta}} \omega_{Q}\right)\left(\tilde{P} X_{\alpha}\right)=\left\langle\beta, \tilde{P} X_{\alpha}\right\rangle
\end{aligned}
$$

which implies

$$
\sharp_{n h}(\alpha)=\tilde{P}\left(X_{\alpha}\right) \text {. }
$$

Furthermore, the symplectic structure $\Omega_{p}$ on $\mathcal{C}_{p}$ at any point $p \in M$ is given by the restriction of the canonical symplectic structure $\omega_{Q}$ on $T^{*} Q$ to $\mathcal{C}_{p}$.

Proposition 4. Let $\gamma: Q \rightarrow M$ be a section of $\pi_{Q_{\mid M}}: M \longrightarrow Q$, then $\operatorname{Im}(\gamma)$ is a lagrangian submanifold of $\left(M, \Lambda_{n h}\right)$ if and only if $d \gamma(X, Y)=0$ for all $X, Y \in D$.

Proof. We notice that $F=\left\{v \in T\left(T^{*} Q\right)\right.$ such that $\left.T \pi_{Q}(v) \in D\right\}$, and an easy computation in local coordinates shows that $\operatorname{dim}(F \cap T M)=2 \operatorname{dim}(D)$. Thus, we have

$$
T \operatorname{Im}(\gamma) \cap \mathcal{C}=T \gamma(D)
$$

On the other hand, it is clear that our definition of lagrangian submanifold is equivalent to $T \operatorname{Im}(\gamma) \cap \mathcal{C}$ be lagrangian with respect to the simplectic structure $\Omega$ on the vector space $\mathcal{C}$. Since $\Omega$ is the restriction of $\omega_{Q}$, given $X, Y \in D$ we have

$$
\Omega(T \gamma(X), T \gamma(Y))=\Omega_{Q}(T \gamma(X), T \gamma(Y))=d \gamma(X, Y) .
$$

So, after a careful counting of dimensions, we deduce that $\operatorname{Im}(\gamma)$ is lagrangian with respect to $\Lambda_{n h}$ if and only if $d \gamma(X, Y)=0$ for all $X, Y \in D$.

Using this proposition we can recover the Nonholonomic Hamilton-Jacobi Theorem as a consequence of Theorem 2.3 (see $[11,13,21]$ ). 
Theorem 5.2. [Nonholonomic Hamilton-Jacobi] Given a hamiltonian $h: M \rightarrow \mathbb{R}$, and $\gamma$ a 1-from on $Q$ taking values in $M$, such that $d \gamma(X, Y)=0$ for all $X, Y \in D$, then the following conditions are equivalent

1. $\bar{X}_{n h}$ and $\bar{X}_{n h}^{\gamma}$ are $\gamma$-related.

2. $d h \in(T \gamma(D))^{\circ}$ (which is in turns equivalent to $\left.d(h \circ \gamma) \in D^{\circ}\right)$.

We will get a suitable expression for the nonholonomic almost-Poisson tensor $\Lambda_{n h}$ defined on the constraint submanifold $M$ of $T^{*} Q$ (see [24,6]). This local representation can be also used to prove Proposition 4 .

Let us recall that the constraints were defined through a distribution $D$ on $Q$. Let $D^{\prime}$ a complementary distribution of $D$ in $T Q$ and assume that $\left\{X_{\alpha}\right\}, 1 \leq \alpha \leq n-k$ is a local basis of $D$ and that $\left\{Y_{a}\right\}, 1 \leq a \leq k$ is a local basis of $D^{\prime}$. Notice that

$$
\mu^{a}\left(X_{\alpha}\right)=0
$$

where $\left\{\mu^{a}\right\}, 1 \leq a \leq k$ is a local basis of the annihilator $D^{o}$ of $D$.

Next we introduce new coordinates in $T^{*} Q$ as follows:

$$
\tilde{p}_{\alpha}=X_{\alpha}^{i} p_{i}, \tilde{p}_{n-k+a}=Y_{a}^{i} p_{i}
$$

where

$$
X_{\alpha}=X_{\alpha}^{i} \frac{\partial}{\partial q^{i}}, Y_{a}=Y_{a}^{i} \frac{\partial}{\partial q^{i}}
$$

In these new coordinates we deduce that the constraints become

$$
\tilde{p}_{n-k+a}=0 .
$$

Therefore, we can take local coordinates $\left(q^{i}, \tilde{p}_{\alpha}\right)$ on $M$.

A direct computation shows now that the nonholonomic almost-Poisson tensor $\Lambda_{n h}$ on $M$ is given by [6]

$$
\begin{aligned}
& \Lambda_{n h}\left(d q^{i}, d q^{j}\right)=0, \Lambda_{n h}\left(d q^{i}, d \tilde{p}_{\alpha}\right)=X_{\alpha}^{i} \\
& \Lambda_{n h}\left(d \tilde{p}_{\alpha}, d \tilde{p}_{\beta}\right)=X_{\beta}^{i} p_{j} \frac{\partial X_{\beta}^{j}}{\partial q^{i}}-X_{\alpha}^{i} p_{j} \frac{\partial X_{\beta}^{j}}{\partial q^{i}} .
\end{aligned}
$$

Summarizing the above discussion we can apply the results obtained in Section 2 to the hamiltonian system given by $h$ on the almost-Poisson manifold $\left(M, \Lambda_{n h}\right)$.

Assume that $\gamma: Q \longrightarrow M$ is a section of $\pi_{Q_{\mid M}}: M \longrightarrow Q$. Then, we have

$$
\gamma\left(q^{i}\right)=\left(q^{i}, \tilde{\gamma}_{\alpha}\left(q^{i}\right)\right)
$$

Since $\gamma$ is a 1 -form on $Q$ taking values on $M$ we deduce

$$
\gamma\left(q^{i}\right)=\left(q^{i}, \gamma_{i}\left(q^{i}\right)\right)
$$

and since it takes values in $M$ we get

$$
\tilde{\gamma}_{\alpha}=X_{\alpha}^{i} \gamma_{i}
$$

A direct computation from equation (9) gives

$$
\begin{aligned}
& \Lambda_{n h}^{\alpha \beta}+\Lambda_{n h}^{\beta j} \frac{\partial \tilde{\gamma}_{\alpha}}{\partial q^{j}}-\Lambda_{n h}^{\alpha j} \frac{\partial \tilde{\gamma}_{\beta}}{\partial q^{j}} \\
& =X_{\beta}^{i} \gamma_{j} \frac{\partial X_{\alpha}^{j}}{\partial q^{i}}-X_{\alpha}^{i} \gamma_{j} \frac{\partial X_{\beta}^{j}}{\partial q^{i}}-X_{\beta}^{j} \frac{\partial}{\partial q^{j}}\left(X_{\alpha}^{i} \gamma_{i}\right)-X_{\alpha}^{j} \frac{\partial}{\partial q^{j}}\left(X_{\beta}^{i} \gamma_{i}\right) \\
& =X_{\alpha}^{i} X_{\beta}^{j}\left(\frac{\partial \gamma_{j}}{\partial q^{i}}-\frac{\partial \gamma_{i}}{\partial q^{j}}\right)=0 .
\end{aligned}
$$


which can be equivalently written as

$$
d \gamma\left(X_{\alpha}, X_{\beta}\right)=0
$$

Therefore, $\gamma(Q)$ is a lagrangian submanifold of $\left(M, \Lambda_{n h}\right)$ if and only if $d \gamma \in$ $\mathcal{I}\left(D^{o}\right)$, where $\mathcal{I}\left(D^{o}\right)$ denotes the ideal of forms generated by $D^{o}$. Indeed, notice that (18) holds if and only if $d \gamma=\sum_{a} \xi_{a} \wedge \mu^{a}$, for some 1-forms $\xi_{a}$.

5.3. Time dependent systems. In this section we will follow [18] for the decription of time dependent mechanical systems. Now we are going to develop a timedependent version of the previous construction. If we have the fibration $E \rightarrow M$ such that $E$ is equipped with an almost-Poisson structure $\Lambda$, we can construct the following fibration in the obvious way

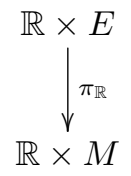

where now $\mathbb{R} \times E$ is equiped with the almost-Poisson structure $\tilde{\Lambda}$ obtained in a natural way extending $\Lambda$ in the trivial manner, that is, if $f$ is a function on $\mathbb{R} \times E$, then $\tilde{\sharp}(d f)=\sharp\left(d f_{0}\right)$, where $f_{0}: E \longrightarrow \mathbb{R}$ is defined by $f_{0}(p)=f(0, p)$, and where we are using the natural identifications for the tangent and cotangent vector spaces of product manifolds. Here, $\sharp=\sharp_{\Lambda}$ and $\sharp=\sharp_{\tilde{\Lambda}}$.

We can consider the "extended" version of this diagram, that is, consider $T^{*} \mathbb{R} \times E$, equipped with the almost-Poisson structure $\Lambda_{\text {ext }}$ given by the addition of the canonical Poisson structure on $T^{*} \mathbb{R}$ and $\Lambda$. Notice that if we consider global coordinates $(t, e)$ on $T^{*} \mathbb{R} \cong \mathbb{R} \times \mathbb{R}$, then

$$
\Lambda_{e x t}=\frac{\partial}{\partial t} \wedge \frac{\partial}{\partial e}+\Lambda
$$

and the canonical projection is

$$
\begin{aligned}
\mu: T^{*} \mathbb{R} \times E & \longrightarrow \mathbb{R} \times E \\
(t, e, p) & \rightarrow \mu(t, e, p)=(t, p) .
\end{aligned}
$$

According to the above notation, diagram (19) becomes

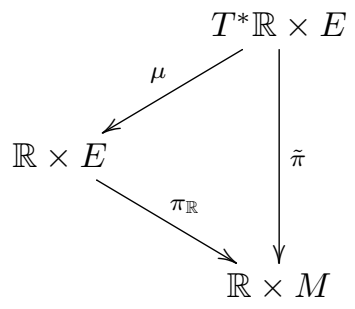

where $\tilde{\pi}=\pi_{\mathbb{R}} \circ \mu$.

Given a time dependent hamiltonian $h: \mathbb{R} \times E \rightarrow \mathbb{R}$, the dynamics are given by the evolution vector field $\frac{\partial}{\partial t}+X_{h} \in \mathfrak{X}(\mathbb{R} \times E)$. We can introduce the extended hamiltonian $h_{\text {ext }}: T^{*} \mathbb{R} \times E \rightarrow \mathbb{R}$ given by $h_{\text {ext }}=\mu^{*} h+e$ and the respective hamiltonian vector field $X_{h_{e x t}}=\sharp_{\Lambda_{e x t}}\left(d h_{e x t}\right)$. Notice that $\mu_{*}\left(X_{h_{e x t}}\right)=\frac{\partial}{\partial t}+X_{h}$.

We will denote by $\mathcal{C}_{e x t}$ the characteristic distribution of $\Lambda_{\text {ext }}$. Notice that $\mathcal{C}_{e x t}(t, e, p)=\left\langle\frac{\partial}{\partial t}, \frac{\partial}{\partial e}\right\rangle+\mathcal{C}_{p}$, under the obvious identifications. 
If $\gamma$ is a section of $\tilde{\pi}$, we can consider the section of $\pi_{\mathbb{R}}$ given by $\mu \circ \gamma$ and define the vector field $\left(\frac{\partial}{\partial t}+X_{h}\right)^{\gamma}$ on $\mathbb{R} \times M$ as follows:

$$
\left(\frac{\partial}{\partial t}+X_{h}\right)^{\gamma}=T \pi_{\mathbb{R}} \circ\left(\frac{\partial}{\partial t}+X_{h}\right) \circ(\mu \circ \gamma)
$$

Now, we can state the time-dependent version of Theorem 2.3.

Theorem 5.3. If $\operatorname{Im}(\gamma)$ is a lagrangian manifold in $\left(T^{*} \mathbb{R} \times E, \Lambda_{\text {ext }}\right)$, then the following assertions are equivalent.

1. $\left(\frac{\partial}{\partial t}+X_{h}\right)$ and $\left(\frac{\partial}{\partial t}+X_{h}\right)^{\gamma}$ are $\mu \circ \gamma$-related

2. $d h_{\text {ext }} \in\left(T \operatorname{Im}(\gamma) \cap \mathcal{C}_{\text {ext }}\right)^{\circ}+\langle d t\rangle$

Proof. "(i) $\Rightarrow$ (ii)"

Assume that $\left(\frac{\partial}{\partial t}+X_{h}\right)$ and $\left(\frac{\partial}{\partial t}+X_{h}\right)^{\gamma}$ are $\mu \circ \gamma$-related, which means that given $m \in M$, then

$$
T \mu \circ T \gamma\left(\left(\frac{\partial}{\partial t}+X_{h}\right)^{\gamma}(m)\right)=\left(\frac{\partial}{\partial t}+X_{h}\right)(\mu \circ \gamma(m))
$$

or equivalently, there exists $B \in \mathbb{R}$ such that

$$
T \gamma\left(\left(\frac{\partial}{\partial t}+X_{h}\right)^{\gamma}(m)\right)=\left(X_{h_{e x t}}+B \frac{\partial}{\partial e}\right)(\gamma(m))
$$

since any tangent vector in $T_{\gamma(m)}\left(T^{*} \mathbb{R} \times E\right)$ which projects by $\mu$ onto $\frac{\partial}{\partial t}+X_{h}$ is of the form

$$
X_{h_{e x t}}+B \frac{\partial}{\partial e}, \quad B \in \mathbb{R} .
$$

Using the same argument that we used in Theorem 2.3 we can conclude that

$$
d h_{\text {ext }}(\gamma(m))+B d t \in\left(T_{\gamma(m)} \operatorname{Im}(\gamma) \cap \mathcal{C}_{\text {ext }}(\gamma(m))\right)^{\circ}
$$

and so

$$
d h_{\text {ext }} \in\left(T \operatorname{Im}(\gamma) \cap \mathcal{C}_{\text {ext }}\right)^{\circ}+\langle d t\rangle .
$$

"(ii) $\Leftarrow($ i)"

Assume that $d h_{e x t} \in\left(T \operatorname{Im}(\gamma) \cap \mathcal{C}_{e x t}\right)^{\circ}+\langle d t\rangle$; therefore, given any point $u \in \operatorname{Im}(\gamma)$, there exists a real number $B$ such that

$$
d h_{e x t}(u)+B d t(u) \in\left(T_{x} \operatorname{Im}(\gamma) \cap\left(\mathcal{C}_{e x t}\right)_{u}\right)^{\circ} .
$$

Now we can deduce

$$
\sharp_{\Lambda_{e x t}}\left(d h_{e x t}(u)+B d t(u)\right) \in T_{u} \operatorname{Im}(\gamma),
$$

where $\sharp_{\Lambda_{\text {ext }}}\left(d h_{\text {ext }}(u)+B d t(u)\right)=X_{h_{\text {ext }}}(u)+B \frac{\partial}{\partial e}(u)$

Obviously, the last statement implies that $T \mu_{*}\left(X_{h_{\text {ext }}}(u)+B \frac{\partial}{\partial e}(u)\right) \in T_{\mu(x)} \operatorname{Im}(\mu \circ$ $\gamma)$, but

$$
\begin{aligned}
T \mu_{*}\left(X_{h_{e x t}}(u)+B \frac{\partial}{\partial e}(u)\right) & =T \mu_{*}\left(X_{h_{e x t}}(u)\right)+T \mu_{*}\left(B \frac{\partial}{\partial e}(u)\right) \\
& =T \mu_{*}\left(X_{h_{e x t}}(u)\right)=\left(\frac{\partial}{\partial t}+X_{h}\right)(\mu(u))
\end{aligned}
$$

wich implies that $\left(\frac{\partial}{\partial t}+X_{h}\right)$ and $\left(\frac{\partial}{\partial t}+X_{h}\right)^{\gamma}$ are $\mu \circ \gamma$-related. 
5.4. External forces. In this section we will apply the above general scheme to time-dependent systems and systems with external forces (see $[9,5])$.

A force is represented by a semi-basic 1 -form $F\left(t, v_{q}\right)=\alpha_{i}(t, q, \dot{q}) d q^{i}$, wich is equivalent to give a bundle mappping

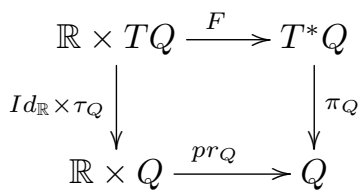

(see [9] for details). Assuming that our dynamical system is described by an hyperregular lagrangian $L: T Q \longrightarrow \mathbb{R}$ and the force $F$, then using the Legendre transformation $F L: T Q \rightarrow \mathbb{R}$ we can transport $F$ to the hamiltonian side and define $\tilde{F}=F \circ(\mathbb{F} L)^{-1}$.

We have

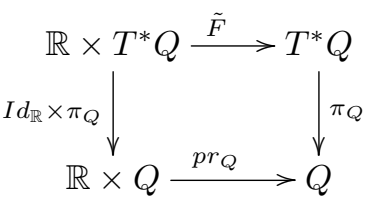

where $\operatorname{pr}_{Q}(t, q)=q$

Given a hamiltonian $h: \mathbb{R} \times T^{*} Q \rightarrow \mathbb{R}$, then the evolution of the system with external force $\tilde{F}$ is now given by

$$
\frac{\partial}{\partial t}+X_{h}+V_{\tilde{F}}
$$

where $V_{\tilde{F}}$ is the vector field determined by

$$
V_{\tilde{F}}\left(t, \alpha_{q}\right)=\sharp_{\Lambda_{Q}}\left(\pi_{Q}^{*}\left(\tilde{F}\left(t, \alpha_{Q}\right)\right)\right),
$$

$\Lambda_{Q}$ being the canonical Poisson structure on $T^{*} Q$.

In bundle coordinates $\frac{\partial}{\partial t}+X_{h}+V_{\tilde{F}}$ provides the differential equation

$$
\begin{aligned}
\dot{q}_{i} & =\frac{\partial h}{\partial p_{i}} \\
\dot{p}_{i} & =-\frac{\partial h}{\partial q_{i}}-\tilde{F}_{i} .
\end{aligned}
$$

We can equip $T^{*}(\mathbb{R} \times Q)$ with the almost-Poisson structure $\tilde{\Lambda}$ given by $\tilde{\Lambda}=$ $\Lambda_{\mathbb{R} \times Q}+V_{F} \wedge \frac{\partial}{\partial e}$ (recall the definition of $e$ in the previous section). Here $\Lambda_{\mathbb{R} \times Q}$ denotes the canonical Poisson tensor on $T^{*}(\mathbb{R} \times Q)$.

In local coordinates

$$
\tilde{\Lambda}=\tilde{F}_{i} \frac{\partial}{\partial e} \wedge \frac{\partial}{\partial p_{i}}+\frac{\partial}{\partial t} \wedge \frac{\partial}{\partial e}+\frac{\partial}{\partial q^{i}} \wedge \frac{\partial}{\partial p_{i}} .
$$

It is easy to see that the characteristic distribution of $\tilde{\Lambda}$ is the whole space. Indeed, using the local expression of $\tilde{\Lambda}$ we have

$$
\begin{aligned}
& \sharp_{\tilde{\Lambda}}(d t)=-\frac{\partial}{\partial e} \\
& \sharp_{\tilde{\Lambda}}(d e)=-F_{i} \frac{\partial}{\partial p_{i}}+\frac{\partial}{\partial t} \\
& \sharp_{\tilde{\Lambda}}\left(d q^{i}\right)=-\frac{\partial}{\partial p^{i}} \\
& \sharp_{\tilde{\Lambda}}\left(d p_{i}\right)=\frac{\partial}{\partial q_{i}}+F_{i} \frac{\partial}{\partial e} .
\end{aligned}
$$


We can define $h_{e x t}=\mu^{*} h+e$, where $\mu$ is defined in the same way that in 20, and construct the hamiltonian vector field $X_{h_{e x t}}=\sharp_{\tilde{\Lambda}}\left(d h_{\text {ext }}\right)$. Due to the definition of $\tilde{\Lambda}$ it is easy to see that $\mu_{*}\left(X_{h_{e x t}}\right)=\frac{\partial}{\partial t}+X_{h}+V_{\tilde{F}}$.

The following diagram summarizes our construction

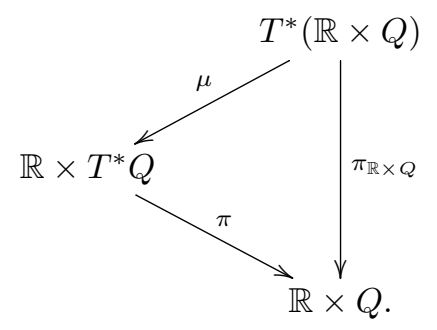

If $\gamma$ is a section of $\pi_{\mathbb{R} \times Q}$ (a 1 -form on $\mathbb{R} \times Q$ ) we can consider the section of $\pi$ given by $\mu \circ \gamma$ and define the vector field $\left(\frac{\partial}{\partial t}+X_{h}+V_{\tilde{F}}\right)^{\gamma}$ on $\mathbb{R} \times M$

$$
\left(\frac{\partial}{\partial t}+X_{h}+V_{\tilde{F}}\right)^{\gamma}=T \pi \circ\left(\frac{\partial}{\partial t}+X_{h}+V_{F}\right) \circ(\mu \circ \gamma)
$$

and we can state the following.

Theorem 5.4. If $\operatorname{Im}(\gamma)$ is a lagrangian manifold in $\left(\left(T^{*}(\mathbb{R} \times Q), \tilde{\Lambda}\right)\right.$, then the following assertions are equivalent.

1. $\left(\frac{\partial}{\partial t}+X_{h}+V_{\tilde{F}}\right)$ and $\left(\frac{\partial}{\partial t}+X_{h}+V_{\tilde{F}}\right)^{\gamma}$ are $\mu \circ \gamma$-related

2. $d h_{\text {ext }} \in T \operatorname{Im}(\gamma)^{\circ}+\langle d t\rangle$

Proof. The proof is analogous to that in Theorem 5.3.

Next, we shall characterize when a section $\gamma$ is lagrangian.

Proposition 5. Let $\gamma$ be a 1-form on $\mathbb{R} \times Q$; then the image of $\gamma$ is a lagrangian submanifold with respect to $\tilde{\Lambda}$ if and only if

$$
d \gamma=(\tilde{F} \circ \mu \circ \gamma) \wedge d t
$$

Proof. Using (21), it is easy to see that $\sharp_{\tilde{\Lambda}}$ is an isomorphism, and so we can define the corresponding almost-symplectic structure $\tilde{\Omega}$, that is $\left(\sharp_{\tilde{\Lambda}}\right)^{-1}=b_{\tilde{\Omega}}$, and thus

$$
\begin{aligned}
& b_{\tilde{\Omega}}\left(\frac{\partial}{\partial t}\right)=-\tilde{F}_{i} d q^{i}+d e \\
& b_{\tilde{\Omega}}\left(\frac{\partial}{\partial e}\right)=-d t \\
& b_{\tilde{\Omega}}\left(\frac{\partial}{\partial q^{i}}\right)=d p_{i}+\tilde{F}_{i} d t \\
& b_{\tilde{\Omega}}\left(\frac{\partial}{\partial p_{i}}\right)=-d q^{i} .
\end{aligned}
$$

Here $b_{\tilde{\Omega}}$ denotes the induced mapping from tangent vector to 1 -forms defined by the 2 -form $\tilde{\Omega}$.

So we can conclude that

$$
\tilde{\Omega}=d q^{i} \wedge d p_{i}+d t \wedge d e+\tilde{F}_{i} d q_{i} \wedge d t .
$$

The image of the 1-form $\gamma$ will be lagrangian for $\tilde{\Omega}$ if and only if

$$
\begin{aligned}
0 & =\gamma^{*}(\tilde{\Omega})=\gamma^{*}\left(d q^{i} \wedge d p_{i}+d t \wedge d e+\tilde{F}_{i} d q_{i} \wedge d t\right) \\
& =\gamma^{*}\left(d q^{i} \wedge d p_{i}+d t \wedge d e\right)+\gamma^{*}\left(\tilde{F}_{i} d q_{i} \wedge d t\right) \\
& =-d \gamma+\left(\tilde{F}_{i} \circ \mu \circ \gamma\right) d q^{i} \wedge d t
\end{aligned}
$$

and the result follows. 
Remark 1. Our result generalizes the Hamilton-Jacobi theorem derived in [3] for the case of linear forces and time-dependent systems [20].

\section{Examples.}

6.1. Hamilton-Jacobi equation for the nonholonomic particle. Let a particle of unit mass be moving in space $Q=\mathbb{R}^{3}$, with lagrangian

$$
L=K-V=\frac{1}{2}\left(\dot{x}^{2}+\dot{y}^{2}+\dot{z}^{2}\right)-V(x, y, z),
$$

and subject to the constraint

$$
\Phi=\dot{z}-y \dot{x}=0 .
$$

Passing to the hamiltonian point of view we define the hamiltonian

$$
h\left(x, y, z, p_{x}, p_{y}, p_{z}\right)=\frac{1}{2}\left(p_{x}^{2}+p_{y}^{2}+p_{z}^{2}\right)+V(x, y, z),
$$

and the submanifold $M$ of $T^{*} Q$ given by $p_{z}-y p_{x}=0$. We have the following decomposition along $M$

$$
T\left(T^{*} Q\right)_{\mid M}=T M \oplus F^{\perp}
$$

where

$$
F^{\perp}=\operatorname{span}\left\{\frac{\partial}{\partial p_{z}}-y \frac{\partial}{\partial p_{x}}\right\} .
$$

Therefore, the projector $P: T\left(T^{*} Q\right)_{\mid M} \longrightarrow T M$ is given by

$$
P=\operatorname{id}_{T\left(T^{*} Q\right)_{\mid M}}-\frac{1}{1+y^{2}}\left(\frac{\partial}{\partial p_{z}}-y \frac{\partial}{\partial p_{x}}\right) \otimes\left(d p_{z}-y d p_{x}-p_{x} d y\right)
$$

and the solution of the nonholonomic dynamics is the following vector field

$$
\begin{aligned}
\bar{X}_{h}= & P\left(X_{h}\right) \\
= & p_{x} \frac{\partial}{\partial x}+p_{y} \frac{\partial}{\partial y}+p_{z} \frac{\partial}{\partial z}-\frac{1}{1+y^{2}}\left(\frac{\partial V}{\partial x}+y \frac{\partial V}{\partial z}+y p_{x} p_{y}\right) \frac{\partial}{\partial p_{x}} \\
& -\frac{\partial V}{\partial y} \frac{\partial}{\partial p_{y}}-\frac{1}{1+y^{2}}\left(y \frac{\partial V}{\partial x}+y^{2} \frac{\partial V}{\partial z}-p_{x} p_{y}\right) \frac{\partial}{\partial p_{z}}
\end{aligned}
$$

restricted to $M$.

Taking noncanonical coordinates $\left(x, y, z, \tilde{p}_{1}, \tilde{p}_{2}, \tilde{p}_{3}\right)$ on $T^{*} Q$ where $\tilde{p}_{1}=p_{x}+y p_{z}$, $\tilde{p}_{2}=p_{y}$ and $\tilde{p}_{3}=p_{z}-y p_{x}$ then $\left(x, y, z, \tilde{p}_{1}, \tilde{p}_{2}\right)$ are coordinates for $M$. Therefore, the nonholonomic bracket defined on $M$ is given by

$$
\begin{aligned}
\left\{x, \tilde{p}_{1}\right\}_{n h} & =1, \quad\left\{y, \tilde{p}_{2}\right\}_{n h}=1, \quad\left\{z, \tilde{p}_{1}\right\}_{n h}=y, \\
\left\{\tilde{p}_{1}, \tilde{p}_{2}\right\}_{n h} & =\frac{1}{1+y^{2}},
\end{aligned}
$$

and the remaining brackets are zero.

Let $\gamma: \mathbb{R}^{3} \rightarrow M$ be a section of $\pi_{Q_{\mid M}}, \operatorname{Im}(\gamma)$ is a lagrangian submanifold of $\left(M, \Lambda_{n h}\right)$ if and only if

$$
d \gamma\left(\frac{\partial}{\partial x}+y \frac{\partial}{\partial z}, \frac{\partial}{\partial y}\right)=0
$$

with $\gamma_{3}-y \gamma_{1}=0$. If $\gamma=\gamma_{1} d x+\gamma_{2} d y+y \gamma_{1} d z$ then this condition is equivalent to

$$
\left(1+y^{2}\right) \frac{\partial \gamma_{1}}{\partial y}+y \gamma_{1}-\frac{\partial \gamma_{2}}{\partial x}-y \frac{\partial \gamma_{2}}{\partial z}=0
$$


The nonholonomic Hamilton-Jacobi equations are in this case

$$
\begin{aligned}
& \gamma_{1} \frac{\partial \gamma_{1}}{\partial x}+\gamma_{2} \frac{\partial \gamma_{2}}{\partial x}+\gamma_{3} \frac{\partial \gamma_{3}}{\partial x}+y\left(\gamma_{1} \frac{\partial \gamma_{1}}{\partial z}+\gamma_{2} \frac{\partial \gamma_{2}}{\partial z}+\gamma_{3} \frac{\partial \gamma_{3}}{\partial z}\right)+\frac{\partial V}{\partial x}+y \frac{\partial V}{\partial z}=0 \\
& \gamma_{1} \frac{\partial \gamma_{1}}{\partial y}+\gamma_{2} \frac{\partial \gamma_{2}}{\partial y}+\gamma_{3} \frac{\partial \gamma_{3}}{\partial y}+\frac{\partial V}{\partial y}=0 .
\end{aligned}
$$

Since the distribution generated by $\left\{X_{1}=\frac{\partial}{\partial x}+y \frac{\partial}{\partial z}, X_{2}=\frac{\partial}{\partial y}\right\}$ is completely nonholonomic, i.e. span $\left\{X_{1}, X_{2},\left[X_{1}, X_{2}\right]\right\}=T Q$, then the nonholonomic Hamilton Jacobi equations can be written in the form:

$$
\frac{1}{2}\left(\left(1+y^{2}\right) \gamma_{1}^{2}+\gamma_{2}^{2}\right)+V(x, y, z)=\text { constant }
$$

with

$$
\left(1+y^{2}\right) \frac{\partial \gamma_{1}}{\partial y}+y \gamma_{1}-\frac{\partial \gamma_{2}}{\partial x}-y \frac{\partial \gamma_{2}}{\partial z}=0
$$

In the case $V \equiv 0$, then the solutions are $\gamma_{1}=\frac{k_{1}}{\sqrt{1+y^{2}}}$ and $\gamma_{2}=k_{2}$, where $k_{1}, k_{2}$ are constants.

Thus, the map

$$
\begin{aligned}
\Phi: \quad & =\mathbb{R}^{2} \\
\left(x, y, x, k_{1}, k_{2}\right) & \longmapsto\left(x, y, z, \frac{k_{1}}{\sqrt{1+y^{2}}}, k_{2}, \frac{y k_{1}}{\sqrt{1+y^{2}}}\right)
\end{aligned}
$$

is a complete solution of the nonholonomic problem. Therefore it is only necessary to integrate the vector field

$$
\begin{aligned}
\dot{x} & =\frac{k_{1}}{\sqrt{1+y^{2}}} \\
\dot{y} & =k_{2} \\
\dot{z} & =\frac{y k_{1}}{\sqrt{1+y^{2}}}
\end{aligned}
$$

to obtain all the solutions of the nonholonomic problem.

6.2. Complete Hamilton-Jacobi equation for the snakeboard. The configuration manifold that modelizes the snakeboard (see figure) is $Q=S E(2) \times \mathbb{T}^{2}$ with coordinates $(x, y, \theta, \psi, \phi)$.

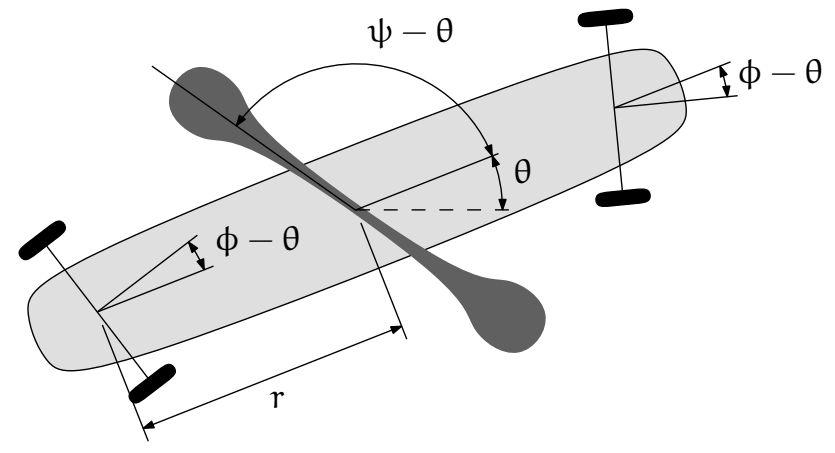

The system is described by a Lagrangian

$$
L(q, \dot{q})=\frac{1}{2} m\left(\dot{x}^{2}+\dot{y}^{2}\right)+\frac{1}{2}\left(J+2 J_{1}\right) \dot{\theta}^{2}+\frac{1}{2} J_{0}(\dot{\theta}+\dot{\psi})^{2}+J_{1} \dot{\phi}^{2}
$$


where $m$ is the total mass of the board, $J>0$ is the moment of inertia of the board, $J_{0}>0$ is the moment of inertia of the rotor of the snakeboard mounted on the body's center of mass and $J_{1}>0$ is the moment of inertia of each wheel axles. The distance between the center of the board and the wheels is denoted by $r$. For simplicity, we assume that $J+J_{0}+2 J_{1}=m r^{2}$.

Since the wheels are not allowed to slide in the sideways direction, we impose the constraints

$$
\begin{aligned}
& -\dot{x} \sin (\theta+\phi)+\dot{y} \cos (\theta+\phi)-r \dot{\theta} \cos \phi=0 \\
& -\dot{x} \sin (\theta-\phi)+\dot{y} \cos (\theta-\phi)+r \dot{\theta} \cos \phi=0 .
\end{aligned}
$$

To avoid singularities of the distribution defined by the previous constraints we will assume, in the sequel, that $\phi \neq \pm \pi / 2$ and the remaining brackets are zero.

In [13], the authors, using the $S E(2)$-symmetry of the problem, show that this nonholonomic problem is described by a vector subbundle $E$ of $T^{*} \mathbb{T}^{2} \times \mathbb{R}^{3} \rightarrow \mathbb{T}^{2}$. This vector subbundle is equipped with coordinates $\left(\phi, \psi, \tilde{p}_{1}, \tilde{p}_{2}, \tilde{p}_{3}\right)$ and and almostPoisson determined by the bracket relations:

$$
\begin{gathered}
\left\{\phi, \tilde{p}_{1}\right\}_{n h}=\frac{1}{\sqrt{2 J_{1}}}, \quad\left\{\psi, \tilde{p}_{2}\right\}_{n h}=\frac{1}{\sqrt{f(\phi)}} \\
\left\{\tilde{p}_{1}, \tilde{p}_{2}\right\}_{n h}=\frac{J_{0} \cos \phi}{r \sqrt{2 J_{1} m f(\phi)}} \tilde{p}_{3} \\
\left\{\tilde{p}_{1}, \tilde{p}_{3}\right\}_{n h}=-\frac{J_{0} \cos \phi}{r \sqrt{2 J_{1} m f(\phi)}} \tilde{p}_{2}
\end{gathered}
$$

where $f(\phi)=J_{0}-\frac{J_{0}^{2} \sin ^{2} \phi}{m r^{2}}$. The hamiltonian of the nonholonomic system is now written as

$$
h\left(\phi, \psi, \tilde{p}_{1}, \tilde{p}_{2}, \tilde{p}_{3}\right)=\frac{1}{2}\left(\tilde{p}_{1}^{2}+\tilde{p}_{2}^{2}+\tilde{p}_{3}^{2}\right) .
$$

A section $\gamma$ of $E \rightarrow \mathbb{T}^{2}$, that is,

$$
(\phi, \psi) \rightarrow\left(\phi, \psi, \gamma_{1}(\phi, \psi), \gamma_{2}(\phi, \psi), \gamma_{3}(\phi, \psi)\right)
$$

verifies that $\operatorname{Im}(\gamma)$ is Lagrangian if and only if verifies Equations (9); that is,

$$
\begin{aligned}
0 & =\frac{J_{0} \cos \phi}{r \sqrt{2 J_{1} m f(\phi)}} \gamma_{3}+\frac{1}{\sqrt{2 J_{1}}} \frac{\partial \gamma_{2}}{\partial \phi}-\frac{1}{\sqrt{f(\phi)}} \frac{\partial \gamma_{1}}{\partial \psi} \\
0 & =\frac{1}{\sqrt{2 J_{1}}} \frac{\partial \gamma_{3}}{\partial \phi}-\frac{J_{0} \cos \phi}{r \sqrt{2 J_{1} m f(\phi)}} \gamma_{2} \\
0 & =\frac{1}{\sqrt{f(\phi)}} \frac{\partial \gamma_{3}}{\partial \psi} .
\end{aligned}
$$

In order to find solutions of the Hamilton-Jacobi equation, we can try to solve the equation $h \circ \gamma=$ constant, that is

$$
\left(\gamma_{1}(\phi, \psi)\right)^{2}+\left(\gamma_{2}(\phi, \psi)\right)^{2}+\left(\gamma_{3}(\phi, \psi)\right)^{2}=\text { constant }
$$

A complete solution of the Hamilton-Jacobi equation is given by the map

$$
\begin{aligned}
\mathbb{T}^{2} \times \mathbb{R}^{3} & \longrightarrow E \\
\left(\phi, \psi, \lambda_{1}, \lambda_{2}, \lambda_{3}\right) & \longmapsto\left(\phi, \psi, \Phi_{1}, \Phi_{2}, \Phi_{3}\right)
\end{aligned}
$$


where

$$
\begin{aligned}
\Phi_{1} & =\lambda_{1} \sqrt{2 J_{1}} \\
\Phi_{2} & =\lambda_{2} \sqrt{f(\phi)}+\frac{J_{0} \lambda_{3}}{r \sqrt{m}} \sin \phi \\
\Phi_{3} & =\frac{J_{0} \lambda_{2}}{r \sqrt{m}} \sin \phi-\lambda_{3} \sqrt{f(\phi)}
\end{aligned}
$$

and the functions

$$
\begin{aligned}
f_{1}\left(\phi, \psi, \tilde{p}_{1}, \tilde{p}_{2}, \tilde{p}_{3}\right) & =\frac{1}{\sqrt{2 J_{1}}} \tilde{p}_{1} \\
f_{2}\left(\phi, \psi, \tilde{p}_{1}, \tilde{p}_{2}, \tilde{p}_{3}\right) & =\frac{1}{J_{0}}\left(\tilde{p}_{2} \sqrt{f(\phi)}+\frac{J_{0} \tilde{p}_{3}}{r \sqrt{m}} \sin \phi\right) \\
f_{3}\left(\phi, \psi, \tilde{p}_{1}, \tilde{p}_{2}, \tilde{p}_{3}\right) & =\frac{1}{J_{0}}\left(\frac{J_{0} \tilde{p}_{2}}{r \sqrt{m}} \sin \phi-\tilde{p}_{3} \sqrt{f(\phi)}\right)
\end{aligned}
$$

are in involution.

\section{REFERENCES}

[1] R. Abraham and J. E. Marsden, Foundations of Mechanics, 2nd ed., Benjamin-Cummings, Reading (Ma), 1978.

[2] V. I. Arnold, Mathematical Methods of Classical Mechanics, Second edition. Graduate Texts in Mathematics, 60. Springer-Verlag, New York, 1989.

[3] P. Balseiro, J. C. Marrero, D. Martín de Diego and E. Padrón, A unified framework for mechanics: Hamilton-Jacobi equation and applications, Nonlinearity, 23 (2010), 1887-1918.

[4] L. Bates and J. Sniatycki, Nonholonomic reduction, Rep. Math. Phys., 32 (1993), 99-115.

[5] F. Cantrijn, Vector fields generating invariants for classical dissipative systems, J. Math. Phys., 23 (1982), 1589-1595.

[6] F. Cantrijn, M. de León and D. Martín de Diego, On almost-Poisson structures in nonholonomic mechanics, Nonlinearity, 12 (1999), 721-737.

[7] J. F. Cariñena, X. Gracia, G. Marmo, E. Martínez, M. Muñoz-Lecanda and N. Román-Roy, Geometric Hamilton-Jacobi theory, Int. J. Geom. Meth. Mod. Phys., 3 (2006), 1417-1458.

[8] J. F. Cariñena, X. Gracia, G. Marmo, E. Martínez, M. Muñoz-Lecanda and N. Román-Roy, Geometric Hamilton-Jacobi theory for nonholonomic dynamical systems, Int. J. Geom. Meth. Mod. Phys., 7 (2010), 431-454.

[9] C. Godbillon, Géométrie Différentielle et Mécanique Analytique, Hermann, Paris, 1969.

[10] M. Leok, T. Ohsawa and D. Sosa, Hamilton-Jacobi Theory for Degenerate Lagrangian Systems with Holonomic and Nonholonomic Constraints, Journal of Mathematical Physics, 53 (2012), 072905 (29 pages).

[11] M. de León, D. Iglesias-Ponte and D. Martín de Diego, Towards a Hamilton-Jacobi theory for nonholonomic mechanical systems, Journal of Physics A: Math. Gen., 41 (2008), 015205, 14 pp.

[12] M. de León, J. C. Marrero and D. Martín de Diego, A geometric Hamilton-Jacobi theory for classical field theories, In: Variations, geometry and physics, 129-140, Nova Sci. Publ., New York, (2009).

[13] M. de León, J. C. Marrero and D. Martín de Diego, Linear almost Poisson structures and Hamilton-Jacobi equation. Applications to nonholonomic mechanics, J. Geom. Mech., 2 (2010), 159-198.

[14] M. de León, D. Martín de Diego, J. C. Marrero, M. Salgado and S. Vilariño, Hamilton-Jacobi theory in $k$-symplectic field theories, Int. J. Geom. Meth. Mod. Phys., 7 (2010), 1491-1507.

[15] M. de León, J. C. Marrero, D. Martín de Diego and M. Vaquero, A Hamilton-Jacobi theory for singular lagrangian systems, J. Math. Phys., 54 (2013), 032902, 32 pp.

[16] M. de León, D. Martín de Diego and M. Vaquero, A Hamilton-Jacobi theory for singular lagrangian systems in the Skinner and Rusk setting, Int. J. Geom. Meth. Mod. Phys., 9 (2012), 1250074, 24 pp. 
[17] M. de León, D. Martín de Diego, C. Martínez-Campos and M. Vaquero, A Hamilton-Jacobi theory in infinite dimensional phase spaces, In preparation.

[18] M. de León and P. R. Rodrigues, Methods of differential geometry in analytical mechanics, North-Holland Mathematics Studies, 158. North-Holland Publishing Co., Amsterdam, 1989.

[19] P. Libermann and Ch.M- Marle, Symplectic Geometry and Analytical Mechanics, D. Reidel Publishing Co., Dordrecht, 1987.

[20] J. C. Marrero and D. Sosa, The Hamilton-Jacobi equation on Lie affgebroids, Int. J. Geom. Methods Mod. Phys., 3 (2006), 605-622.

[21] T. Oshawa and A. M. Bloch, Nonholonomic Hamilton-Jacobi equations and integrability, J. Geom. Mech., 1 (2009), 461-481.

[22] H. Rund, The Hamilton-Jacobi Theory in the Calculus of Variations, Hazell, Watson and Viney Ltd., Aylesbury, Buckinghamshire, U.K. 1966.

[23] I. Vaisman, Lectures on the Geometry of Poisson Manifolds, Progress in Mathematics, 118. Birkhäuser Verlag, Basel, 1994.

[24] A. J. van der Schaft and B. M. Maschke, On the Hamiltonian formulation of nonholonomic mechanical systems, Rep. Math. Phys., 34 (1994), 225-233.

Received November 2012; revised January 2014.

E-mail address: mdeleon@icmat.es

E-mail address: david.martin@icmat.es

E-mail address: miguel.vaquero@icmat.es 


\section{A|P $\left.\right|_{\substack{\text { Journal of } \\ \text { Mathematical Physics }}}$}

\section{On the Hamilton-Jacobi theory for singular lagrangian systems}

Manuel de León, Juan Carlos Marrero, David Martín de Diego, and Miguel Vaquero

Citation: Journal of Mathematical Physics 54, 032902 (2013); doi: 10.1063/1.4796088

View online: http://dx.doi.org/10.1063/1.4796088

View Table of Contents: http://scitation.aip.org/content/aip/journal/jmp/54/3?ver=pdfcov

Published by the AIP Publishing

Articles you may be interested in

Incompleteness of the Hamilton-Jacobi theory

Am. J. Phys. 82, 848 (2014); 10.1119/1.4876355

Hamilton-Jacobi theory for degenerate Lagrangian systems with holonomic and nonholonomic constraints

J. Math. Phys. 53, 072905 (2012); 10.1063/1.4736733

Jacobi's last multiplier and Lagrangians for multidimensional systems

J. Math. Phys. 49, 073517 (2008); 10.1063/1.2956486

3-geometries and the Hamilton-Jacobi equation

J. Math. Phys. 45, 2543 (2004); 10.1063/1.1753667

Intrinsic characterization of the variable separation in the Hamilton-Jacobi equation

J. Math. Phys. 38, 6578 (1997); 10.1063/1.532226

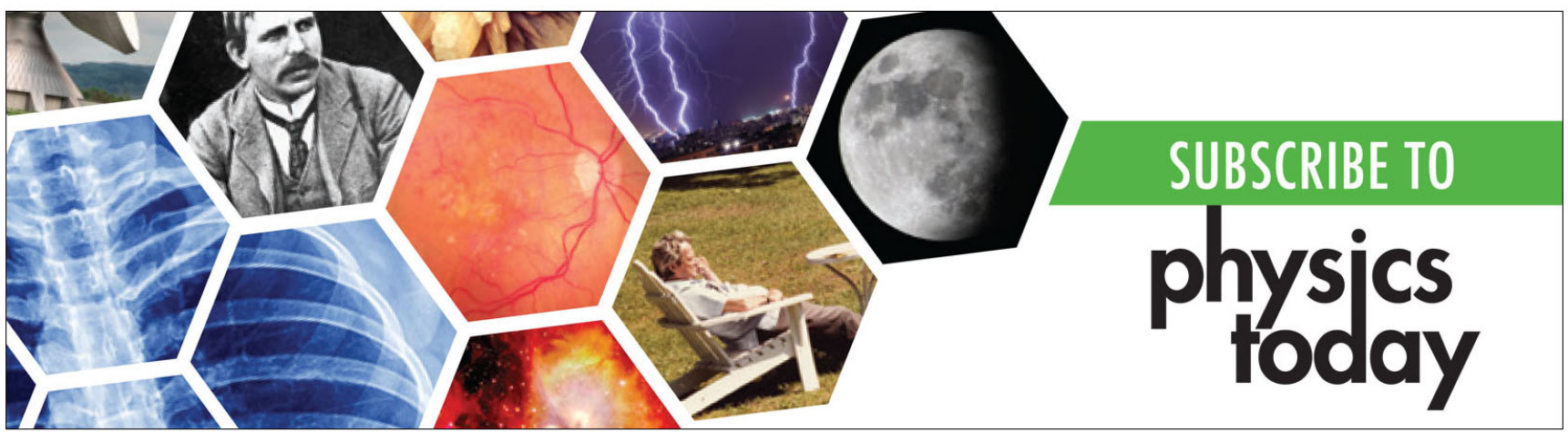




\title{
On the Hamilton-Jacobi theory for singular lagrangian systems
}

\author{
Manuel de León, ${ }^{1, a)}$ Juan Carlos Marrero, ${ }^{2, b)}$ David Martín de Diego, ${ }^{1, c)}$ \\ and Miguel Vaquero ${ }^{1, d)}$ \\ ${ }^{1}$ Instituto de Ciencias Matemáticas (CSIC-UAM-UC3M-UCM), C/ Nicolás Cabrera, \\ n. 13-15, Campus Cantoblanco, UAM 28049 Madrid, Spain \\ ${ }^{2}$ Unidad Asociada ULL-CSIC "Geometría Diferencial y Mecánica Geométrica," \\ Departamento de Matemática Fundamental, Universidad de La Laguna, Tenerife, \\ Canary Islands, Spain
}

(Received 10 May 2012; accepted 7 March 2013; published online 26 March 2013)

We develop a Hamilton-Jacobi theory for singular lagrangian systems using the Gotay-Nester-Hinds constraint algorithm. The procedure works even if the system has secondary constraints. (C) 2013 American Institute of Physics. [http://dx.doi.org/10.1063/1.4796088]

\section{INTRODUCTION}

One of the most classical problems of theoretical mechanics is the study of constrained systems. Essentially, there are two different meanings to understand constrained systems. One refers to systems where we externally impose constraints allowing some particular motions (external constraints). The second case is when the degeneracy of a lagrangian function imposes constraints on the phase space of the system (internal constraints). In this paper, we will restrict ourselves to this last situation.

At a first step, when the lagrangian is singular, there appear constraints restricting the admissible positions and velocities (primary constraints). Later on, the evolution of these initial constraints may produce new constraints (called secondary constraints).

The theory of degenerate (or singular) lagrangian systems is relevant in Field theory, and just the quantization of these systems led to Dirac and Bergmann ${ }^{4,10}$ to develop a wonderful theory of constraints (the Dirac-Bergmann theory of constraints), later geometrized by Gotay et al... 14,16,18,20 This topic has deserved a lot of work by many authors (see, for instance, Sundermeyer ${ }^{41}$ and Marmo et $a l .{ }^{34}$ ) Recently, Leok et $a l .{ }^{23}$ have studied degenerate lagrangians arising from truly mechanical systems, even in presence of additional nonholonomic constraints (see also the paper by de León and Martín de Diego ${ }^{28}$ ). We would also like to mention two papers by Eden ${ }^{11,12}$ where the author proposed a quantization procedure for nonholonomic mechanical systems; in some extent, in these two papers it is introduced a preliminary version of the nowadays called nonholonomic bracket.

Another important topic in theoretical mechanics is the Hamilton-Jacobi theory which allows us to find solutions of a hamiltonian system by means of solutions of a partial differential equation, the Hamilton-Jacobi equation. Conversely, we can treat to solve a PDE using the characteristic curves of a Hamiltonian system (see these two standard books ${ }^{1,2}$ and the $\operatorname{paper}^{7}$ for a general view of the theory and some modern approaches in terms of lagrangian submanifolds; see also Ref. 38 for a more classical view). In Ref. 35 we have discussed the Hamilton-Jacobi equation for time-dependent Hamiltonian systems in the setting of Lie affgebroids; in Refs. 24 and 26, we have successfully extended the classical Hamilton-Jacobi theory for nonholonomic systems (see also Refs. 8 and 36); and in Refs. 25 and 29 for classical field theories. Therefore, it seems quite relevant

\footnotetext{
a) E-mail: mdeleon@icmat.es

b) E-mail: jcmarrer@ull.es

c)E-mail: david.martin@icmat.es

d) E-mail: miguel.vaquero@icmat.es
} 
to extend the Hamilton-Jacobi theory also for degenerate lagrangian systems, and this is just the goal of the present paper. As it is appointed by Bergmann" "the Hamilton-Jacobi formalism merits special attention, because of its affinity to quantum theory." Indeed a complete solution of the HamiltonJacobi equation generates a canonical transformation that are analogues to unitary transformations in Hilbert space, and particular solutions may be linked to individual quantum states, we refer to Ref. 4 for the details.

Briefly, the standard formulation of the Hamilton-Jacobi problem is to find a function $S\left(t, q^{A}\right)$ (called the principal function) such that

$$
\frac{\partial S}{\partial t}+H\left(q^{A}, \frac{\partial S}{\partial q^{A}}\right)=0 .
$$

If we put $S\left(t, q^{A}\right)=W\left(q^{A}\right)-t E$, where $E$ is a constant, then $W$ satisfies

$$
H\left(q^{A}, \frac{\partial W}{\partial q^{A}}\right)=E,
$$

where $W$ is called the characteristic function. Equations (1.1) and (1.2) are indistinctly referred as the Hamilton-Jacobi equations.

There have been several attempts to develop a Hamilton-Jacobi theory for degenerate lagrangian systems. ${ }^{31,32,37}$ These procedures were based on the homogenization of the given lagrangian, which leads to a new lagrangian system with null energy; then, it is possible to discuss the Hamilton-Jacobi equation for the constraints themselves. The main problem is that, due to the integrability condition for the resultant partial differential equation, one can only consider first class constraints. Therefore, the treatment of the cases when second class constraints appear should be developed by ad hoc arguments (as in Ref. 37, for instance). Thus, in Refs. 31 and 32 the authors only discuss the case of primary constraints.

Therefore, the Hamilton-Jacobi problem for degenerate lagrangian systems is far to be solved.

Our procedure to develop a geometric Hamilton-Jacobi theory is strongly inspired in two main issues. The first one in the recent approach to the Hamilton-Jacobi theory developed by Cariñena et $\mathrm{al}^{7}$ (see also Refs. 8, 9, 33 and 26 for the applications to nonholonomic mechanics and field theory); and the second one, is the geometric theory of constraints due to Gotay and Nester. ${ }^{20}$

Let us recall that given an almost regular lagrangian $L: T Q \rightarrow \mathbb{R}$ one can define a presymplectic system on $M_{1}=F L(T Q) \subset T^{*} Q$, the primary constraint submanifold where $\omega_{1}$ is the restriction of the canonical symplectic form on $T^{*} Q$ to $M_{1}$, and $F L: T Q \rightarrow T^{*} Q$ is the Legendre transformation defined by $L$. The dynamics is obtained from the equation

$$
i_{X} \omega_{1}=d h_{1},
$$

where $h_{1} \in C^{\infty}\left(M_{1}\right)$ is the projection of the energy $E_{L} \in C^{\infty}(T Q)$.

The above equation produces a sequence of submanifolds

$$
\cdots M_{k} \hookrightarrow \cdots \hookrightarrow M_{2} \hookrightarrow M_{1} \hookrightarrow T^{*} Q
$$

and, eventually, a final constraint submanifold $M_{f}$ if the algorithm stabilizes at some step.

The strategy is to consider the projection of the constraint submanifolds provided by the constraint algorithm, so that we obtain new surjective submersions onto submanifolds of the given configuration manifold. This fact permits to connect a given solution of the final constraint submanifolds $M_{f}$, with its projection onto $Q_{f}\left(\pi_{f}: M_{f} \rightarrow Q_{f}\right.$ is the surjective submersion) using a section of $\pi_{f}$.

The second order differential equation (SODE) problem is also discussed such that one can obtain the corresponding lagrangian picture.

We also discuss the relation of the geometric Hamilton-Jacobi problem with the HamiltonJacobi problem (in a traditional sense) for arbitrary extensions of $h_{1}$, in terms of first and second class primary and secondary constraints. Therefore, this work can be considered as the natural extension to the Hamilton-Jacobi problem of the geometrization by Gotay and Nester ${ }^{20}$ of the Dirac constraint algorithm.

Several examples are discussed along the paper in order to illustrate the theory. 


\section{CLASSICAL HAMILTON-JACOBI THEORY (GEOMETRIC VERSION)}

The standard formulation of the Hamilton-Jacobi problem is to find a function $S\left(t, q^{A}\right)$ (called the principal function) such that

$$
\frac{\partial S}{\partial t}+h\left(q^{A}, \frac{\partial S}{\partial q^{A}}\right)=0,
$$

where $h=h\left(q^{A}, p_{A}\right)$ is the hamiltonian function of the system. If we put $S\left(t, q^{A}\right)=W\left(q^{A}\right)-t E$, where $E$ is a constant, then $W$ satisfies

$$
h\left(q^{A}, \frac{\partial W}{\partial q^{A}}\right)=E,
$$

where $W$ is called the characteristic function.

Equations (2.1) and (2.2) are indistinctly referred as the Hamilton-Jacobi equation.

Let $Q$ be the configuration manifold, and $T^{*} Q$ its cotangent bundle equipped with the canonical symplectic form,

$$
\omega_{Q}=d q^{A} \wedge d p_{A},
$$

where $\left(q^{A}\right)$ are coordinates in $Q$ and $\left(q^{A}, p_{A}\right)$ are the induced ones in $T^{*} Q$. In what follows, $\pi_{Q}: T^{*} Q \longrightarrow Q$ will denote the canonical projection.

Let $h: T^{*} Q \longrightarrow \mathbb{R}$ a hamiltonian function and $X_{h}$ the corresponding hamiltonian vector field, say

$$
i_{X_{h}} \omega_{Q}=d h .
$$

Therefore, the integral curves $\left(q^{A}(t), p_{A}(t)\right)$ of $X_{h}$ satisfy the Hamilton equations:

$$
\frac{d q^{A}}{d t}=\frac{\partial h}{\partial p_{A}}, \frac{d p_{A}}{d t}=-\frac{\partial h}{\partial q^{A}} .
$$

We can define also the Poisson bracket of two functions. Given $f$ and $g$ real functions on $T^{*} Q$, we define a new function $\{f, g\}$ by

$$
\{f, g\}=\omega_{Q}\left(X_{f}, X_{g}\right),
$$

where $X_{f}$ and $X_{g}$ are the corresponding hamiltonian vector fields.

The Poisson bracket gives us the evolution of observables, since given the hamiltonian $h$ we have

$$
\dot{f}=X_{h}(f)=i_{X_{h}}\left(i_{X_{f}} \omega_{Q}\right)=\omega_{Q}\left(X_{f}, X_{h}\right)=\{f, h\},
$$

and then we can rewrite the Hamilton equations as

$$
\begin{aligned}
& \dot{q}^{A}=\left\{q^{A}, h\right\}, \\
& \dot{p}_{A}=\left\{p_{A}, h\right\} .
\end{aligned}
$$

Let $\lambda$ be a closed 1 -form on $Q$, say $d \lambda=0$; (then, locally $\lambda=d W$ ).

The following theorem gives us the relation of the Hamilton-Jacobi equation and the solutions of the Hamilton equations (see Refs. 1 and 2).

Theorem 2.1. The following conditions are equivalent:

(i) If $\sigma: I \rightarrow Q$ satisfies the equation

$$
\frac{d q^{A}}{d t}=\frac{\partial h}{\partial p_{A}} \circ \lambda,
$$

then $\lambda \circ \sigma$ is a solution of the Hamilton equations;

(ii) $d(h \circ \lambda)=0$. 
We can reinterpret Theorem 2.1 as follows (see Refs. 7, 24, and 26).

Define a vector field on $Q$ :

$$
X_{h}^{\lambda}=T \pi_{Q} \circ X_{h} \circ \lambda .
$$

The following diagram illustrates the construction of the vector field $X_{h}^{\lambda}$ :

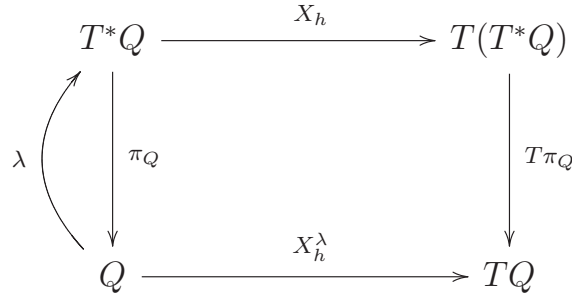

Then the following conditions are equivalent:

(i) If $\sigma: I \rightarrow Q$ satisfies the equation

$$
\frac{d q^{A}}{d t}=\frac{\partial h}{\partial p_{A}},
$$

then $\lambda \circ \sigma$ is a solution of the Hamilton equations;

(i) ${ }^{\prime} \quad$ If $\sigma: I \rightarrow Q$ is an integral curve of $X_{h}^{\lambda}$, then $\lambda \circ \sigma$ is an integral curve of $X_{h}$;

(i)" $X_{h}$ and $X_{h}^{\lambda}$ are $\lambda$-related, i.e.,

$$
T \lambda\left(X_{h}^{\lambda}\right)=X_{h} \circ \lambda
$$

Next, we have the following intrinsic version of Theorem 2.1.

Theorem 2.2. Let $\lambda$ be a closed 1-form on $Q$. Then the following conditions are equivalent:

(i) $\quad X_{h}^{\lambda}$ and $X_{h}$ are $\lambda$-related;

(ii) $\quad d(h \circ \lambda)=0$.

Proof: In local coordinates, we have that

$$
X_{h}=\frac{\partial h}{\partial p_{A}} \frac{\partial}{\partial q^{A}}-\frac{\partial h}{\partial q^{A}} \frac{\partial}{\partial p_{A}}
$$

and

$$
\lambda=\lambda_{A}(q) d q^{A}
$$

Then,

$$
\begin{aligned}
X_{h}^{\lambda} & =\frac{\partial h}{\partial p_{A}}(\lambda(q)) \frac{\partial}{\partial q^{A}}, \\
T \lambda\left(X_{h}^{\lambda}\right) & =\frac{\partial h}{\partial p_{A}} \frac{\partial}{\partial q^{A}}+\frac{\partial h}{\partial p_{A}} \frac{\partial \lambda_{B}}{\partial q^{A}} \frac{\partial}{\partial p_{B}}, \\
d(h \circ \lambda) & =\left(\frac{\partial h}{\partial q^{A}}+\frac{\partial h}{\partial p_{B}} \frac{\partial \lambda_{B}}{\partial q^{A}}\right) d q^{A} .
\end{aligned}
$$

Since $d \lambda=0$ if and only if

$$
\frac{\partial \lambda_{A}}{\partial q^{B}}=\frac{\partial \lambda_{B}}{\partial q^{A}},
$$

we have the equivalences between (i) and (ii).

If

$$
\lambda=\lambda_{A}(q) d q^{A}
$$


then the Hamilton-Jacobi equation becomes

$$
h\left(q^{A}, \lambda_{A}\left(q^{B}\right)\right)=\text { const } .
$$

If $\lambda=d W$ then we recover the classical formulation

$$
h\left(q^{A}, \frac{\partial W}{\partial q^{A}}\right)=\text { const } .
$$

since

$$
\lambda_{A}=\frac{\partial W}{\partial q^{A}} .
$$

\section{THE HAMILTON-JACOBI THEORY IN THE LAGRANGIAN SETTING}

Let $L: T Q \longrightarrow \mathbb{R}$ be a lagrangian function, that is,

$$
L=L\left(q^{A}, \dot{q}^{A}\right),
$$

where $\left(q^{A}, \dot{q}^{A}\right)$ denotes the induced coordinates on the tangent bundle $T Q$ of the configuration manifold $Q$. In what follows, $\tau_{Q}: T Q \longrightarrow Q$ will denote the canonical projection.

Let us denote by

$$
S=d q^{A} \otimes \frac{\partial}{\partial \dot{q}^{A}}
$$

and

$$
\Delta=\dot{q}^{A} \frac{\partial}{\partial \dot{q}^{A}}
$$

the vertical endomorphism and the Liouville vector field on $T Q$ (see Ref. 30 for intrinsic definitions).

The Poincaré-Cartan 2-form is defined by

$$
\omega_{L}=-d \alpha_{L}, \alpha_{L}=S^{*}(d L)
$$

and the energy function

$$
E_{L}=\Delta(L)-L,
$$

which in local coordinates read as

$$
\begin{aligned}
\alpha_{L} & =\hat{p}_{A} d q^{A}, \\
\omega_{L} & =d q^{A} \wedge d \hat{p}_{A}, \\
E_{L} & =\dot{q}^{A} \hat{p}_{A}-L(q, \dot{q}),
\end{aligned}
$$

where $\hat{p}_{A}=\frac{\partial L}{\partial \dot{q}^{A}}$ stand for the generalized momenta. Here $S^{*}$ denotes the adjoint operator of $S$.

The lagrangian $L$ is said to be regular if the Hessian matrix

$$
\left(W_{A B}=\frac{\partial^{2} L}{\partial \dot{q}^{A} \partial \dot{q}^{B}}\right)
$$

is regular, and in this case, $\omega_{L}$ is a symplectic form on $T Q$.

We define the Legendre transformation as a fibred mapping $F L: T Q \longrightarrow T^{*} Q$ such that

$$
\left\langle F L\left(v_{q}\right), u_{q}\right\rangle=\left\langle\tilde{X}_{v_{q}}, \alpha_{L}\left(v_{q}\right)\right\rangle,
$$

where $T \tau_{Q}\left(\tilde{X}_{v_{q}}\right)=u_{q} \in T_{q} Q$. In local coordinates we get

$$
F L\left(q^{A}, \dot{q}^{A}\right)=\left(q^{A}, \hat{p}_{A}\right),
$$

and $L$ is regular if and only if $F L$ is a local diffeomorphism. 
If $L$ is regular, then there exist a unique vector field $\xi_{L}$ on $T Q$ satisfying the symplectic equation

$$
i_{\xi_{L}} \omega_{L}=d E_{L}
$$

and, moreover, it automatically satisfies the second order differential equation (SODE) condition, i.e.,

$$
S \xi_{L}=\Delta
$$

\section{A. Second order differential equations}

A first order differential equation can be geometrically interpreted as a vector field $X$ on a manifold. Indeed in a local coordinate system $\left(x^{i}\right)$, we can write

$$
X=X^{i}(x) \frac{\partial}{\partial x^{i}}
$$

and, therefore, a curve $\gamma(t)=\left(x^{i}(t)\right)$ in $M$ is an integral curve of $X$, i.e.,

$$
\dot{\gamma}(t)=X(\gamma(t)) \text {, for all } \mathrm{t},
$$

if and only if the following system of differential equations

$$
\frac{d x^{i}}{d t}=X^{i}(x(t))
$$

holds.

A second order differential equation can be geometrically interpreted as a vector field $Y$ on the tangent bundle $T M$ of a manifold $M$. Indeed, let $Y$ be a tangent vector on $T M$, and take induced coordinates $\left(x^{i}, v^{i}\right)$ on $T M$. We consider a particular kind of vector field $Y$ in $T M$; those such that the projection of its integral curves coincide with the tangent lifts of these projections. That is, if $\gamma(t)=\left(x^{i}(t), v^{i}(t)\right)$ is an integral curve of $Y$, then its projection is the curve $\left(\tau_{M} \circ \gamma\right)(t)=x^{i}(t)$ and the corresponding tangent lift of $\tau_{M} \circ \gamma$ is

$$
\overbrace{\tau_{M} \circ \gamma} \circ(t)=\left(x^{i}(t), \frac{d x^{i}}{d t}\right) .
$$

This property is equivalent to say that $Y$ has the following local expression:

$$
Y(x, v)=v^{i} \frac{\partial}{\partial x^{i}}+Y^{i}(x, v) \frac{\partial}{\partial v^{i}} .
$$

Therefore, the curve $\tau_{M} \circ \gamma$ satisfies the system of second order differential equations

$$
\begin{aligned}
& v^{i}=\frac{d x^{i}}{d t}, \\
& \frac{d v^{i}}{d t}=\frac{d^{2} x^{i}}{d t}=Y^{i}\left(x, \frac{d x}{d t}\right) .
\end{aligned}
$$

Such a vector field $Y$ is called a SODE, and the curves $\tau_{M} \circ \gamma$ are just its solutions.

A simple computation shows that a vector field $Y$ on $T M$ is a SODE if and only if $S Y=\Delta$.

Assume now that $L$ is hyperregular, that is, $F L: T Q \rightarrow T^{*} Q$ is a global diffeomorphism; then we can define a (global) hamiltonian function $h: T^{*} Q \rightarrow \mathbb{R}$ by $h=E_{L} \circ F L^{-1}$. It is easy to show that $F L^{*} \omega_{Q}=\omega_{L}$ and that $\xi_{L}$ and, then $X_{h}$ are $F L$-related. So, the solutions of the Euler-Lagrange equations transform by $F L$ into solutions of the Hamilton equations and viceversa. Given a vector field $Z$ on $Q$ we define a new vector field on $Q$ by

$$
\xi_{L}^{Z}=T \tau_{Q} \circ \xi_{L} \circ Z
$$


that is, we have the following commutative diagram

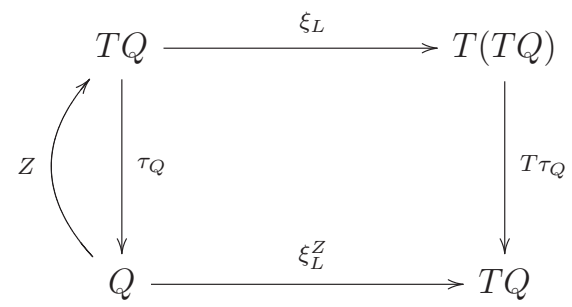

Since $\xi_{L}$ and $X_{h}$ are $F L$-related, it follows that $\xi_{L}^{Z}=X_{h}^{F L \circ Z}$. Thus, Theorem 2.2 can be reformulated as follows.

Theorem 3.1. Let $Z$ be a vector field on $Q$ such that $F L \circ Z$ is a closed 1 -form. Then the following conditions are equivalent:

(i) $\xi_{L}^{Z}$ and $\xi_{L}$ are $Z$-related;

(ii) $\quad d\left(E_{L} \circ Z\right)=0$.

Proof: The result follows as a direct consequence of Theorem 2.2.

\section{THE HAMILTON-JACOBI THEORY FOR SINGULAR LAGRANGIAN SYSTEMS}

In this section we shall give a geometric approach to the Hamilton-Jacobi theory in terms of the Gotay-Nester-Hinds constraint algorithm. ${ }^{16,17}$

Let $L: T Q \longrightarrow \mathbb{R}$ be a singular lagrangian, that is, the Hessian matrix

$$
\left(W_{A B}=\frac{\partial^{2} L}{\partial \dot{q}^{A} \partial \dot{q}^{B}}\right)
$$

is not regular, or, equivalently, the closed 2-form $\omega_{L}$ is not symplectic.

Therefore, the equation

$$
i_{\xi} \omega_{L}=d E_{L}
$$

has no solution in general, or the solutions are not defined everywhere. Moreover, the solutions do not necessarily satisfy the SODE condition. Recall that SODE condition is

$$
S \xi=\Delta
$$

or, equivalently,

$$
T \tau_{Q}(X)=\tau_{T Q}(X),
$$

where $\tau_{Q}: T Q \rightarrow Q$ and $\tau_{T Q}: T T Q \rightarrow T Q$ are the canonical projections. This condition means that the tangent lifts of the projections of the integral curves of $\xi$ to $T Q$ (i.e., the solutions of $\xi$ ) coincide with the integral curves (see Ref. 13).

Singular lagrangian systems have been extensively studied by Dirac and Bergmann, ${ }^{10}$ in order to obtain a procedure for canonical quantization of local gauge theories. They developed an algorithm (called Dirac-Bergmann theory of constraints) that has been later geometrized by Gotay and Nester. ${ }^{16,17,20}$

Definition 4.1. In the sequel, we will assume that $L$ is almost regular, which means that:

- $M_{1}=F L(T Q)$ is a submanifold of $T^{*} Q$;

- The restriction of the Legendre mapping $F L_{1}: T Q \longrightarrow M_{1}$ is a submersion with connected fibers.

The submanifold $M_{1}$ is called the submanifold of primary constraints. 
If $L$ is almost regular, since $\operatorname{ker}(T F L)=\operatorname{ker}\left(\omega_{L}\right) \cap V(T Q)$, where $V(T Q)$ denotes the vertical bundle, and the fibers are connected then a direct computation shows that $E_{L}$ projects onto a function

$$
h_{1}: M_{1} \longrightarrow \mathbb{R} \text {. }
$$

Denote by $j_{1}: M_{1} \longrightarrow T^{*} Q$ the natural inclusion and define

$$
\omega_{1}=j_{1}^{*}\left(\omega_{Q}\right)
$$

Consider now the equation

$$
i_{X} \omega_{1}=d h_{1}
$$

There are two possibilities:

- There is a solution $X$ defined at all the points of $M_{1}$; such $X$ is called a global dynamics and it is a solution (modulo ker $\omega_{1}$ ). In other words, there are only primary constraints. Denote also by $\omega_{1}^{b}: T M_{1} \rightarrow T^{*} M_{1}$ the bundle map induced by the 2-form $\omega_{1}$ on $M_{1}$. Then, since $\operatorname{Im}\left(\omega_{1}\right)$ $=\operatorname{ker}\left(\omega_{1}\right)^{\circ}$ we have that $d h_{1} \in \operatorname{Im}\left(\omega_{1}\right) \Leftrightarrow d h_{1} \in\left(\operatorname{ker}\left(\omega_{1}\right)\right)^{\circ}$ and (4.3) will have solution if and only if $d h_{1}$ annihilates the kernel of $\omega_{1}$. Therefore, taking a local basis of $\operatorname{ker}\left(\omega_{1}\right)$ will permit us to describe explicitly the constraint functions.

- Otherwise, we select the submanifold $M_{2}$ formed by those points of $M_{1}$ where a solution exists. But such a solution $X$ is not necessarily tangent to $M_{2}$, so we have to impose an additional tangency condition, and we obtain a new submanifold $M_{3}$ along which there exists a solution. Continuing this process, we obtain a sequence of submanifolds

$$
\cdots M_{k} \hookrightarrow \cdots \hookrightarrow M_{2} \hookrightarrow M_{1} \hookrightarrow T^{*} Q,
$$

where the general description of $M_{l+1}$ is

$$
\begin{aligned}
& M_{l+1}:=\left\{p \in M_{l} \text { such that there exists } X_{p} \in T_{p} M_{l}\right. \text { satisfying } \\
& \left.i_{X} \omega_{1}(p)=d h_{1}(p)\right\} .
\end{aligned}
$$

If the algorithm stabilizes at some $k$, say $M_{k+1}=M_{k}$, then we say that $M_{k}$ is the final constraint submanifold which is denoted by $M_{f}$, and then there exists a well-defined solution $X$ of (4.3) along $M_{f}$. Notice that we are assuming that the sets $M_{l}$ are submanifolds; although this could not be true it holds in several important examples (see Ref. 14).

Remark 4.2. There is another characterization of the submanifolds $M_{l}$ that we will describe now. If $N$ is a submanifold of $M_{1}$ then we define

$$
T N^{\perp}=\left\{Z \in T_{p}\left(M_{1}\right), p \in N \text { such that } \omega_{1}(X, Z)=0 \text { for all } X \in T_{p} N\right\} .
$$

Then, at any point $p \in M_{l}$ there exists $X_{p} \in T_{p} M_{l}$ verifying $i_{X} \omega_{1}(p)=d h_{1}(p)$ if and only if $\left\langle T M_{l}^{\perp}, d h_{1}\right\rangle=0$ (see Ref. 20).

Hence, we can define the $l+1$ step of the constraint algorithm as

$$
M_{l+1}:=\left\{p \in M_{l} \text { such that }\left\langle T M_{l}^{\perp}, d h_{1}\right\rangle(p)=0\right\},
$$

where $T M_{l}^{\perp}$ is defined as above. Again, any local basis of $T M_{f}^{\perp}$ can be used to give an explicit description of the constraints at any step of the algorithm.

\section{A. Case I: There is a global dynamics}

In this case there exists a vector field $X$ on $M_{1}$ such that

$$
\left.\left(i_{X} \omega_{1}=d h_{1}\right)\right|_{M_{1}} .
$$

Moreover, we have $\pi_{1}\left(M_{1}\right)=Q$, where $\pi_{1}$ is the restriction to $M_{1}$ of the canonical projection $\pi_{Q}: T^{*} Q \longrightarrow Q$. 
Next, assume that $\gamma$ is a closed 1 -form on $Q$ such that $\gamma(Q) \subset M_{1}$. Define now a vector field $X^{\gamma}$ on $Q$ by putting

$$
X^{\gamma}=T \pi_{1} \circ X \circ \gamma .
$$

The following diagram summarizes the above construction:

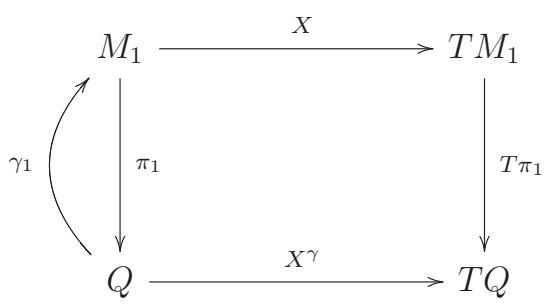

Here $\gamma_{1}$ denotes the restriction of $\gamma$.

We have

$$
\begin{aligned}
\gamma_{1}^{*}\left(i_{X-T \gamma_{1}\left(X^{\gamma}\right)} \omega_{1}\right) & =\gamma_{1}^{*}\left(i_{X} \omega_{1}\right)-\gamma_{1}^{*}\left(i_{T \gamma_{1}\left(X^{\gamma}\right)} \omega_{1}\right) \\
& =\gamma_{1}^{*} d\left(h_{1}\right)-\gamma_{1}^{*}\left(i_{T \gamma_{1}\left(X^{\gamma}\right)} \omega_{1}\right) \\
& =d\left(h_{1} \circ \gamma_{1}\right)
\end{aligned}
$$

since $\gamma^{*}\left(i_{T \gamma_{1} X^{\gamma}} \omega_{1}\right)=i_{X^{\gamma}}\left(\gamma_{1}^{*} \omega_{1}\right)=0$, because

$$
\gamma_{1}^{*} \omega_{1}=\gamma_{1}^{*} j_{1}^{*} \omega_{Q}=\left(j_{1} \circ \gamma_{1}\right)^{*} \omega_{Q}=\gamma^{*} \omega_{Q}=-d \gamma=0 .
$$

Therefore, taking into account that $\left(X \circ \gamma_{1}-T \gamma_{1}\left(X^{\gamma}\right)\right)(q) \in V_{q} \pi_{1}$, for all $q \in Q$, that $V \pi_{1} \oplus$ $T \gamma_{1}(T Q)=T M_{1}$ and $\omega_{1}$ (as it happens with $\omega_{Q}$ ) vanishes acting on two vertical tangent vectors with respect to the canonical projection $\pi_{1}: M_{1} \rightarrow Q$, we deduce the following:

$$
X-T \gamma_{1}\left(X^{\gamma}\right) \in \operatorname{ker}\left(\omega_{1}\right) \Leftrightarrow d\left(h_{1} \circ \gamma_{1}\right)=0 .
$$

Moreover, we will show that it is possible to refine condition (4.4) and to prove that $X$ and $X^{\gamma}$ are $\gamma_{1}$ related.

First of all, it is clear that for any point $p$ of $M_{1}$

$$
T_{p}\left(T^{*} Q\right)=T_{p} M_{1}+V_{p}\left(T^{*} Q\right)
$$

where $V\left(T^{*} Q\right)$ denotes the space of vertical tangent vectors at $p$.

We have that, $X-T \gamma_{1}\left(X^{\gamma}\right)$ is vertical at the points of $\operatorname{Im}\left(\gamma_{1}\right)$, so given any $Z \in V_{p}\left(T^{*} Q\right)$, $p \in \operatorname{Im}\left(\gamma_{1}\right)$, we deduce

$$
\omega_{Q}\left(X-T \gamma_{1}\left(X^{\gamma}\right), Z\right)=0 \text { along } \operatorname{Im}\left(\gamma_{1}\right)
$$

since $\omega_{Q}$ vanishes on two vertical tangent vectors.

Now, given $Z \in T_{p} M_{1}$ we have

$$
\omega_{Q}\left(X-T \gamma_{1}\left(X^{\gamma}\right), Z\right)=\omega_{1}\left(X-T \gamma_{1}\left(X^{\gamma}\right), Z\right)=0
$$

because $X-T \gamma_{1}\left(X^{\gamma}\right) \in \operatorname{ker}\left(\omega_{1}\right)$, and we obtain that $\omega_{Q}\left(X-T \gamma_{1}\left(X^{\gamma}\right), Z\right)=0$ for any tangent vector $Z \in T_{p}\left(T^{*} Q\right)$ on any point $p$ of $\operatorname{Im}\left(\gamma_{1}\right)$. Since $\omega_{Q}$ is non-degenerate we deduce that $X \circ \gamma_{1}$ $=T \gamma_{1}\left(X^{\gamma}\right)$.

In conclusion, we have the following result.

Proposition 4.3.

$$
X \text { and } X^{\gamma} \text { are } \gamma_{1} \text { related } \Leftrightarrow d\left(h_{1} \circ \gamma_{1}\right)=0 \text {. }
$$

Remark 4.4. As a consequence of the above result, if $h_{1}$ is constant along $\gamma_{1}(Q)$ then $\gamma_{1}$ maps the integral curves of $X^{\gamma}$ on integral curves of $X$. So $d\left(h_{1} \circ \gamma_{1}\right)=0$ can be considered as the Hamilton-Jacobi equation in this case. 


\section{B. Case II: There are secondary constraints}

In this case, the algorithm produces a sequence of submanifolds as follows:

$$
\cdots M_{k} \hookrightarrow \cdots \hookrightarrow M_{2} \hookrightarrow M_{1} \hookrightarrow T^{*} Q .
$$

We assume that the projections $Q_{r}:=\pi_{Q}\left(M_{r}\right)$ are submanifolds of $Q$, and that the corresponding projections $\pi_{r}: M_{r} \rightarrow Q_{r}$ are fibrations, where $\pi_{r}$ is the restriction of $\pi_{Q}$ to $M_{r}$.

The constraint algorithm produces a solution $X$ of the equation

$$
\left(i_{X} \omega_{1}=d h_{1}\right)_{\mid M_{f}},
$$

where $X$ is a vector field on $M_{f}$.

Coming back to the Gotay-Nester-Dirac algorithm we can summarize the situation in the following diagram:

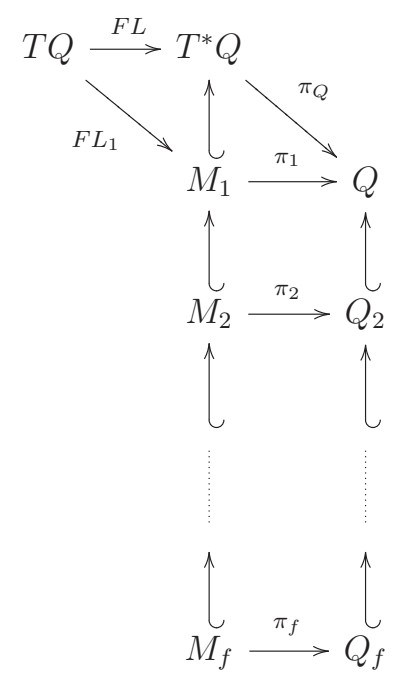

Assume now that $\gamma$ is a closed 1 -form on $Q$ such that

- $\gamma(Q) \subset M_{1}$.

- $\gamma\left(Q_{f}\right) \subset M_{f}$.

As in Case I, $\gamma$ allows us to define a vector field $X^{\gamma} \in \mathfrak{X}\left(Q_{f}\right)$ by

$$
X^{\gamma}=T \pi_{f} \circ X \circ \gamma_{f} .
$$

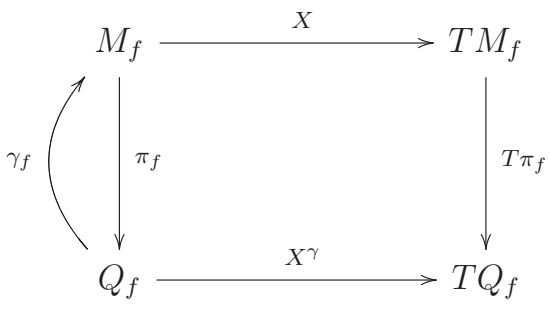

Here $\gamma_{f}$ is the restriction of $\gamma$ to $Q_{f}$.

Now, given $q \in Q_{f}$, we have

$$
\begin{aligned}
& i_{\left(X\left(\gamma_{1}(q)\right)-T_{q} \gamma_{f}\left(X^{\gamma}(q)\right)\right)} \omega_{1} \circ T_{q} \gamma_{1}=i_{X(\gamma(q))} \omega_{1} \circ T_{q} \gamma_{1}-i_{T_{q} \gamma_{f}\left(X^{\gamma}(q)\right)} \omega_{1} \circ T_{q} \gamma_{1} \\
& =d h_{1}\left(\gamma_{f}(q)\right) \circ T_{q} \gamma=d\left(h_{1} \circ \gamma_{1}\right)(q) .
\end{aligned}
$$


Observe that since $\gamma_{f}$ is the restriction of $\gamma_{1}$ we have $T_{q} \gamma_{f}\left(X^{\gamma}(q)\right)=T_{q} \gamma\left(X^{\gamma}(q)\right)$. Therefore, given $Y_{q} \in T_{q} Q$ then $T_{q} \gamma_{1}(Y(q))=T_{q} \gamma(Y(q))$, and we deduce that

$$
\begin{aligned}
& i_{T_{q} \gamma_{f}\left(X^{\gamma}(q)\right)} \omega_{1} \circ T_{q} \gamma\left(Y_{q}\right)=\omega_{1}\left(T_{q} \gamma\left(X^{\gamma}(q)\right), T_{q} \gamma\left(Y_{q}\right)\right) \\
& =\left(\gamma_{1}^{*} \omega_{1}\right)\left(X^{\gamma}(q), Y_{q}\right)=\gamma_{1}^{*} j_{1}^{*} \omega_{Q}\left(X^{\gamma}(q), Y_{q}\right)=d \gamma\left(X^{\gamma}(q), Y_{q}\right)=0 .
\end{aligned}
$$

The previous discussion can be applied to every point $q \in Q_{f}$; therefore, taking into account that $\omega_{1}$ vanishes acting on two vertical tangent vectors and $V \pi_{1} \oplus T \gamma_{1}(T Q)=T M_{1}$, we can deduce the following:

$$
X \circ \gamma_{f}-T \gamma_{f}\left(X^{\gamma}\right) \in \operatorname{ker}\left(\omega_{1}\right)_{\mid \gamma\left(Q_{f}\right)} \Leftrightarrow d\left(h_{1} \circ \gamma_{1}\right)_{\mid Q_{f}}=0 .
$$

Using a similar argument that in Case I, it is possible to deduce that $X$ and $X^{\gamma}$ are $\gamma_{f}$ related since we have

$$
T_{p}\left(T^{*} Q\right)=T_{p} M_{1}+V_{p}\left(T^{*} Q\right)
$$

for all $p \in M_{f}$.

Therefore, we deduce the following.

Proposition 4.5.

$$
X \text { and } X^{\gamma} \text { are } \gamma_{f} \text { related } \Leftrightarrow d\left(h_{1} \circ \gamma_{1}\right)_{Q_{f}}=0 \text {. }
$$

Remark 4.6. Note that the condition $X$ and $X^{\gamma}$ are $\gamma_{f}$-related implies that, if $\sigma: \mathbb{R} \longrightarrow Q_{f}$ is an integral curve of $X^{\gamma}$, then $\sigma_{\gamma}=\gamma \circ \sigma: \mathbb{R} \longrightarrow M_{f}$ is an integral curve of $X$.

Therefore, the condition

$$
d\left(h_{1} \circ \gamma_{1}\right)_{\mid Q_{f}}=0
$$

could be still considered as the Hamilton-Jacobi equation in this context.

\section{Hamilton-Jacobi theory for further geometric constraint equations}

Besides of the equation $i_{X} \omega=d h_{1}$ introduced in Sec. IV B, other equations concerning singular lagrangians have been studied in the literature. For completeness, in this section we will discuss some of these equations of motion.

We refer to Refs. 14,40, and 41 for more details.

\section{Extended equation of motion and the Dirac conjecture}

A constraint is called first class provided its Poisson bracket with every other constraint weakly vanishes, and second class otherwise (see Sec. IV D for more details). Dirac ${ }^{10}$ conjectured that all first-class secondary constraints generate "gauge transformations" which leave the physical state invariant (see, for instance, Ref. 15 and references therein for the discussion about the availability of the Dirac conjecture; moreover, the motivation of our study will be more clear in Sec. IV D).

Without entering in physical discussions, we will analyze if it is possible to extend our HamiltonJacobi formalism for the equations derived assuming Dirac conjecture. Therefore, we need first to discuss the geometry of this "extended equation" for singular lagrangians.

Suppose that we are under the conditions of Sec. IV B. We have $j_{1}: M_{1} \rightarrow T^{*} Q$ where $M_{1}$ is a submanifold and $j_{1}$ the inclusion, and a constrained hamiltonian $h_{1}: M_{1} \rightarrow \mathbb{R}$. As before, we study the presymplectic system $\left(M_{1}, \omega_{1}, d h_{1}\right)$ and apply the Gotay-Nester-Hinds algorithm, assuming that we reach to a final constraint submanifold $M_{f}$. Denote by $j_{f}: M_{f} \rightarrow M_{1}$ the inclusion. Now we say that a vector field $X$ on $M_{f}$ is a solution of the extended equations of motion if $X$ can be written

$$
X=Y+Z \text {, }
$$

where $Y$ and $Z$ are vector fields on $M_{f}$, such that $i_{Y} \omega_{1}=d h_{1}$ and $Z \in \operatorname{ker}\left(\omega_{f}\right)$ where $\omega_{f}:=j_{f}^{*}\left(\omega_{1}\right)$. 
We can now obtain a less restrictive version of the previous Hamilton-Jacobi theory, which gives solutions of the extended equations of motion.

Assume again that $\gamma$ is a 1 -form on $Q$ such that

(i) $\operatorname{Im}(\gamma) \subset M_{1}$,

(ii) $\operatorname{Im}\left(\gamma_{f}\right) \subset M_{f}$,

(iii) $d \gamma=0$.

From a fixed solution $X$ of the extended equation, we can define $X^{\gamma}=T \pi_{f} \circ X \circ \gamma_{f}$.

Proceeding as in Sec. IV B, we have

$$
\begin{aligned}
\gamma_{f}^{*}\left(i_{X-T \gamma_{f}\left(X^{\gamma}\right)} \omega_{f}\right) & =\gamma_{f}^{*}\left(i_{X} \omega_{f}\right)-\gamma_{f}^{*}\left(i_{T \gamma_{f}\left(X^{\gamma}\right)} \omega_{f}\right) \\
& =\gamma_{f}^{*} d h_{1}-\gamma_{f}^{*}\left(i_{T \gamma\left(X^{\gamma}\right)} \omega_{f}\right) \\
& =d\left(h_{1} \circ \gamma_{f}\right) .
\end{aligned}
$$

since $\gamma$ is closed.

Using similar arguments that in Sec. IV B, we deduce the following

Proposition 4.7. Under the above conditions, we have

$$
X \circ \gamma_{f}-T \gamma_{f}\left(X^{\gamma}\right) \in \operatorname{ker}\left(\omega_{f}\right)_{\mid \gamma_{f}\left(Q_{f}\right)} \Leftrightarrow d\left(h_{1} \circ \gamma_{f}\right)=0 .
$$

Proof. It follows the same lines of the proof of Proposition 4.5 but now observing that

$$
T M_{f}=T \gamma_{f}\left(T Q_{f}\right) \oplus V \pi_{f},
$$

and $V \pi_{f} \subset V_{M_{f}} \pi_{Q}$

By the last proposition $T \gamma_{f}\left(X^{\gamma}\right)=X+\tilde{Z}$, with $\tilde{Z} \in \operatorname{ker} \omega_{f}$. Then, from (4.5) we have that $T \gamma_{f}\left(X^{\gamma}\right)=Y+(Z+\tilde{Z})$. So, $T \gamma_{f}\left(X^{\gamma}\right)$ is a solution of the extended equations of motion.

Therefore, the condition

$$
d\left(h_{1} \circ \gamma_{f}\right)=0
$$

could be still considered as the Hamilton-Jacobi equation in this context.

\section{Hinds algorithm}

Besides of the Gotay-Nester-Hinds algorithm, other approaches have been discussed in the literature. In particular we briefly recall the algorithm introduced by Hinds (see Gotay et al. ${ }^{20}$ for a detailed discussion). Hinds algorithm also starts considering the equation $i_{X} \omega_{1}=d h_{1}$ as in the Gotay-Nester-Hinds algorithm. The algorithm generates a descending sequence of constraint submanifolds. In the favorable case, the algorithm stabilizes at a final constraint submanifold which we will denote again by $N_{f}$ (see discussion below). It is important to point out that, in general, this constraint submanifold $N_{f}$ will be different from the final constraint submanifold obtained by the Gotay-Nester-Hinds algorithm, that is $N_{f} \neq M_{f}$. In principle, both algorithms start to diverge from each other after the second step.

In geometric terms, assume that we are in the conditions of Sec. IV C 1 . Define $N_{1}:=M_{1}$ as we did before and consider the following subset $(l>1)$ :

$$
N_{l+1}:=\left\{p \in N_{l} \text { such that exists } X \in T_{p} N_{l} \text { verifying } i_{X} \omega_{l}=d h_{l}\right\},
$$

where, if we call $k_{l}: N_{l} \rightarrow N_{1}$ the natural inclusion, then $\omega_{l}:=k_{l}^{*} \omega_{1}$ and $h_{l}:=k_{l}^{*} h_{1}$. We obtain the sequence of submanifolds

$$
\cdots N_{k} \hookrightarrow \cdots \hookrightarrow N_{2} \hookrightarrow N_{1}=M_{1} \hookrightarrow T^{*} Q .
$$


Again if the algorithm stabilizes, i.e., $N_{k}=N_{k+1}$, then we say that $N_{k}$ is the final constraint manifold, $N_{f}$. In this case, the Hinds algorithm produces a solution $X \in \mathfrak{X}\left(N_{f}\right)$ of the equation

$$
i_{X} \omega_{f}=d h_{f} .
$$

This equation is less restrictive than (4.3), and so the two algorithms diverge for $l \geq 2$. In Ref. 20 we find the following example that illustrates the difference between both algorithms; let $L: T \mathbb{R}^{4} \rightarrow \mathbb{R}$ be

$$
L\left(q^{1}, q^{2}, q^{3}, q^{4}, \dot{q}^{1}, \dot{q}^{2}, \dot{q}^{3}, \dot{q}^{4}\right)=\frac{1}{2}\left(\dot{q}^{1}\right)^{2}-\frac{1}{2}\left(q^{1}\right)^{2}-\left(q^{3} q^{4}\right)+\frac{1}{2}\left(\dot{q}^{4}-q^{2}\right)^{2} .
$$

It is easy to check that in this case the algorithms diverge. In fact, a direct computation shows that the Gotay-Nester-Hinds algorithm stabilizes at $M_{f}=M_{3}$, where $M_{3}$ is given by the constraints

$$
\begin{aligned}
& \Phi_{1}\left(q^{A}, p_{A}\right)=p_{2}, \Phi_{2}\left(q^{A}, p_{A}\right)=p_{3}, \\
& \Phi_{3}\left(q^{A}, p_{A}\right)=p_{4}, \Phi_{4}\left(q^{A}, p_{A}\right)=q^{4}, \\
& \Phi_{5}\left(q^{A}, p_{A}\right)=q^{3}, \Phi_{6}\left(q^{A}, p_{A}\right)=p_{4}+q^{2},
\end{aligned}
$$

while the Hinds algorithm stabilizes at $N_{f}=N_{2}$, where $N_{2}$ is given by the constraints

$$
\begin{aligned}
& \Phi_{1}\left(q^{A}, p_{A}\right)=p_{2}, \Phi_{2}\left(q^{A}, p_{A}\right)=p_{3}, \\
& \Phi_{3}\left(q^{A}, p_{A}\right)=p_{4}, \Phi_{4}\left(q^{A}, p_{A}\right)=q^{4} .
\end{aligned}
$$

Now, we can develop a Hamilton-Jacobi theory in this setting.

Assume that there exists a 1-form $\gamma$ on $Q$ satisfying

(i) $\operatorname{Im}\left(\gamma_{f}\right) \subset N_{f}$,

(ii) $d \gamma=0$ along $N_{f}$.

Then we can define $X^{\gamma}=T \pi_{f} \circ X \circ \gamma_{f}$ and state the equivalent Hamilton-Jacobi theory. The proof follows the same lines that in Proposition 4.7.

Proposition 4.8.

$$
X \circ \gamma_{f}-T \gamma_{f}\left(X^{\gamma}\right) \in \operatorname{ker}\left(\omega_{f}\right)_{\mid \operatorname{Im}\left(\gamma_{f}\right)} \Leftrightarrow d\left(h_{f} \circ \gamma_{f}\right)=0 .
$$

\section{Relation to the Dirac-Bergmann theory of constraints}

In this section we will discuss the relation of the Gotay and Nester theory with the original Dirac-Bergmann theory of constraints.

Assume that we begin with an almost regular lagrangian $L: T Q \rightarrow \mathbb{R}$. Then there exists an open neighbourhood, $U \subset T^{*} Q$ where, in canonical coordinates $\left(q^{A}, p_{A}\right), M_{1} \cap U$ is given by the vanishing of functions $\Phi^{i}\left(q^{A}, p_{A}\right)$ defined on $U$. The functions $\Phi^{i}$ are called primary constraints.

Remember that we can project $E_{L}$ to $h_{1}: M_{1} \rightarrow \mathbb{R}$, and any extension of $h_{1}$ to $U$ should be of the form

$$
H=h+u_{i} \Phi^{i}
$$

where $h$ is an arbitrary extension to $U$ of $h_{1}$. The functions $u_{i}, 1 \leq i \leq 2 \operatorname{dim} Q-\operatorname{dim} M_{1}$ are Lagrange multipliers to be determined.

According to Dirac the equations of motion are

$$
\begin{aligned}
& \dot{q}^{A}=\frac{\partial h}{\partial p_{A}}+u_{i} \frac{\partial \Phi^{i}}{\partial p_{A}}, \\
& \dot{p}_{A}=-\frac{\partial h}{\partial q^{A}}-u_{i} \frac{\partial \Phi^{i}}{\partial q^{A}},
\end{aligned}
$$

which must hold over $U_{1}:=M_{1} \cap U$. If we denote $j_{1}: U_{1} \rightarrow U$ the inclusion, and $\omega_{1}=j_{1}^{*} \omega_{Q}$, the previous equations can be equivalently rewritten as 


$$
i_{X} \omega_{1}=d h_{1} \text { or }\left(i_{X} \omega_{Q}=d h+u_{i} d \Phi^{i}\right)_{\mid U_{1}},
$$

which are the equations that we have considered in the Gotay-Nester-Hinds algorithm.

Since $X$ must be tangent to $U_{1}$ we should have

$$
\begin{aligned}
0 & =\left(X\left(\Phi^{i}\right)\right)_{\mid U_{1}}=\left\{\Phi^{i}, H\right\}_{\mid U_{1}}=\left\{\Phi^{i}, h+u_{j} \Phi^{j}\right\}_{\mid U_{1}} \\
& =\left(\left\{\Phi^{i}, h\right\}+u_{j}\left\{\Phi^{i}, \Phi^{j}\right\}\right)_{\mid U_{1}} .
\end{aligned}
$$

These equations can be trivially satisfied, determine some Lagrange multipliers or add new constraints on the variables $q^{A}, p_{A}$ over $U_{1}$. These new constraints, if any, are called secondary constraints. Suppose that we have obtained the secondary constraints $\varsigma^{\alpha}$. So, we have to restrict the dynamics to $U_{2}:=U_{1} \cap\left(\varsigma^{\alpha}\right)^{-1}\{0\}$.

Again, the solution must be tangent to $U_{2}$ and it requires that

$$
\begin{aligned}
0 & =\left(X\left(\varsigma^{\alpha}\right)\right)_{\mid U_{2}}=\left\{\varsigma^{\alpha}, H\right\}_{\mid U_{2}}=\left\{\varsigma^{\alpha}, h+u_{i} \Phi^{i}\right\}_{\mid U_{2}} \\
& =\left(\left\{\varsigma^{\alpha}, h\right\}+u_{i}\left\{\varsigma^{\alpha}, \Phi^{i}\right\}\right)_{\mid U_{2}} .
\end{aligned}
$$

As before, these equations may determine more Lagrange multipliers or add new constraints to the picture, that is, new secondary constraints. Iterating this procedure, if the algorithm stabilizes, we arrive to a set $U_{f}$ which is an open subset of the final constraint manifold $M_{f}$ obtained by the Gotay-Nester-Hinds algorithm (see Ref. 20 for a proof ).

It is necessary to introduce some definitions. We say that a function defined on $U$ is first class if its Poisson bracket with every constraint (primary and secondary) vanishes. Otherwise, it is said to be of second class (see Sec. IV C 1).

We can reorder constraints into first class or second class. We will denote by $\chi^{a}$ and $\xi^{b}$, the primary first and second class constraints, respectively; and by $\psi^{c}$ and $\theta^{d}$, the secondary first and second class constraints, respectively. We will also denote by $\mu_{a}, \lambda_{b}$ the corresponding Lagrange multipliers for the primary first and second class constraints, respectively.

So, if the problem has a solution, we must obtain a vector field $X$ over $U_{f}$, which satisfies the equations

$$
\left(i_{X} \omega_{Q}=d h+\mu_{a} d \chi^{a}+\lambda_{b} d \xi^{b}\right)_{\mid M_{f}} .
$$

The $\lambda_{b}$ 's are determined functions and the $\mu_{a}$ 's can be varied to obtain other admissible solutions. In consequence, it is also clear that primary first class constraints correspond to gauge transformations which leave the physical state invariant. As we have discussed before, Dirac conjectured that the first class secondary constraints may also generate gauge transformations, therefore, the generalized equations of motion discussed in Subsection IV C 1 are locally rewritten as

$$
\left(i_{X} \omega_{Q}=d h+\mu_{a} d \chi^{a}+\lambda_{b} d \xi^{b}+v_{c} d \psi^{c}\right)_{\mid M_{f}},
$$

where $\lambda_{b}$ are still determined functions and $\mu_{a}$ and $v_{c}$ can be varied arbitrarily. The hamiltonian $h+\mu_{a} \chi^{a}+\lambda_{b} \xi^{b}+v_{c} \psi^{c}$ is called the extended hamiltonian, and Eq. (4.7) the extended equation of motion following the terminology of Ref. 14. Geometrically, the solutions of (4.7) are just

$$
X=Y+Z \text {, }
$$

where $Y$ is a vector field on $M_{f}$ solution of the equations of motion (4.6), and $Z \in \operatorname{ker}\left(\omega_{f}\right)$ where $\omega_{f}$ is the restriction of $\omega_{1}$ to $M_{f}$.

Remark 4.9. If we proceed in the same way with the Hinds algorithm developed in Subsection IV C 2, we will arrive to solutions $X$ satisfying

$$
\left(i_{X} \omega_{Q}=d h+\mu_{a} d \chi^{a}+\lambda_{b} d \xi^{b}+\bar{v}_{\bar{c}} d \bar{\psi}^{\bar{c}}+\bar{w}_{\bar{d}} d \bar{\theta}^{\bar{d}}\right)_{\mid N_{f}},
$$

where $\bar{v}_{\bar{c}}, \bar{w}_{\bar{d}}$ are the Lagrange multipliers corresponding to the constraints $\bar{\psi}^{\bar{c}}$ and $\bar{\theta}^{\bar{d}}$. Note that $\bar{\psi}^{\bar{c}}$ and $\bar{\theta} \bar{d}$ now correspond to the secondary constraints of the final constraint manifold $N_{f}$ in the Hinds algorithm. 


\section{E. Examples}

Now we illustrate the previous propositions with several examples.

\section{There are only primary constraints}

Example 4.10. This example is discussed by Krupkova in Ref. 22. In this example we only have primary first class constraints. Let $L$ be the Lagrangian $L: T \mathbb{R}^{3} \rightarrow \mathbb{R}$ given by

$$
L\left(q^{1}, q^{2}, q^{3}, \dot{q}^{1}, \dot{q}^{2}, \dot{q}^{3}\right)=\frac{1}{2}\left(\dot{q}^{1}+\dot{q}^{2}\right)^{2} .
$$

Then $F L$ is given by $F L: T \mathbb{R}^{3} \rightarrow T^{*} \mathbb{R}^{3}$

$$
F L\left(q^{1}, q^{2}, q^{3}, \dot{q}^{1}, \dot{q}^{2}, \dot{q}^{3}\right)=\left(q^{1}, q^{2}, q^{3}, \dot{q}^{1}+\dot{q}^{2}, \dot{q}^{1}+\dot{q}^{2}, 0\right),
$$

and the primary constraints are

$$
\Phi^{1}\left(q^{A}, p_{A}\right)=p_{1}-p_{2} \Phi^{2}\left(q^{A}, p_{A}\right)=p_{3} .
$$

So

$$
M_{1}=\left\{\left(q^{1}, q^{2}, q^{3}, p_{1}, p_{2}, p_{3}\right) \in \mathbb{R}^{6} \text { such that } p_{1}=p_{2}, p_{3}=0\right\}
$$

and we can use $\left(q^{1}, q^{2}, q^{3}, p_{1}\right)$ as coordinates on $M_{1}$.

It follows that

$$
\begin{aligned}
& E_{L}=\left(\dot{q}^{1}+\dot{q}^{2}\right) \dot{q}^{1}+\left(\dot{q}^{1}+\dot{q}^{2}\right) \dot{q}^{2}-L=L \\
& h_{1}=\frac{1}{2}\left(p_{1}\right)^{2} \\
& \omega_{1}=d q^{1} \wedge d p_{1}+d q^{2} \wedge d p_{1} \\
& \operatorname{ker}\left(\omega_{1}\right)=\left\{\frac{\partial}{\partial q^{3}}, \frac{\partial}{\partial q^{1}}-\frac{\partial}{\partial q^{2}}\right\}
\end{aligned}
$$

and a particular extension of the hamiltonian is

$$
h\left(q^{A}, p_{A}\right)=\frac{1}{2}\left(p_{1}\right)^{2}
$$

It is easy to see that at the points of $M_{1}=\operatorname{Im}(F L)$

$$
\left\{\Phi^{i}, h+u^{1} \Phi^{1}+u^{2} \Phi^{2}\right\}=0, i=1,2 .
$$

So we have global dynamics on $M_{1}$ and it holds that

$$
\left\{\Phi^{1}, \Phi^{2}\right\}=0
$$

and we conclude that there are only first class constraints. The solutions of $\left(i_{X} \omega_{1}=d h_{1}\right)_{\mid M_{1}}$ on $M_{1}$ are given by

$$
X=p_{1} \frac{\partial}{\partial q^{1}}+f_{1} \frac{\partial}{\partial q^{3}}+f_{2}\left(\frac{\partial}{\partial q^{1}}-\frac{\partial}{\partial q^{2}}\right),
$$

where $f_{1}$ and $f_{2}$ are functions on $M_{1}$.

We now look for $\gamma \in \Lambda^{1}(Q)$ such that

(i) $\gamma(Q) \subset M_{1}$,

(ii) $d\left(h_{1} \circ \gamma\right)=0$,

(iii) $d \gamma=0$.

Suppose

$$
\gamma\left(q^{1}, q^{2}, q^{3}\right)=\left(q^{1}, q^{2}, q^{3}, \gamma_{1}\left(q^{1}, q^{2}, q^{3}\right), \gamma_{2}\left(q^{1}, q^{2}, q^{3}\right), \gamma_{3}\left(q^{1}, q^{2}, q^{3}\right)\right),
$$

then, $\gamma(Q) \subset M_{1}$ implies that $\gamma_{1}=\gamma_{2}$ and $\gamma_{3}=0$. 
The condition $d\left(h_{1} \circ \gamma\right)=0$ implies that $\frac{1}{2}\left(\gamma_{1}\right)^{2}=$ constant, and because of that $\gamma_{1}=c$ where $c$ is a constant and so $\gamma_{2}=c$.

Now $\gamma\left(q^{1}, q^{2}, q^{3}\right)=\left(q^{1}, q^{2}, q^{3}, c, c, 0\right)$ and $d \gamma=0$ is trivially satisfied.

If we take the general solution $p_{1} \frac{\partial}{\partial q^{1}}+f_{1} \frac{\partial}{\partial q^{3}}+f_{2}\left(\frac{\partial}{\partial q^{1}}-\frac{\partial}{\partial q^{2}}\right)$ then we obtain $X^{\gamma}=T \pi_{q} \circ X$ $\circ \gamma=c \frac{\partial}{\partial q^{1}}+\left(f_{1} \circ \gamma\right) \frac{\partial}{\partial q^{3}}+\left(f_{2} \circ \gamma\right)\left(\frac{\partial}{\partial q^{1}}-\frac{\partial}{\partial q^{2}}\right)$. If we apply $T \gamma\left(q^{A}\right)\left(X^{\gamma}\left(q^{A}\right)\right)=X\left(\gamma\left(q^{A}\right)\right)$, then we recover the solution $X$ over the points of $\gamma$. It is clear, that integral curves of $X^{\gamma}$ are applied by $\gamma$ into integral curves of $X$ along $\operatorname{Im} \gamma$.

Example 4.11. This example has been discussed by Barcelos-Neto and Braga. ${ }^{3}$ It is an example where only primary second class constraints appear, but it does not exist a solution of the HamiltonJacobi equation. Let $L$ be the Lagrangian $L: T \mathbb{R}^{4} \rightarrow \mathbb{R}$ given by

$$
L\left(q^{1}, q^{2}, q^{3}, q^{4}, \dot{q}^{1}, \dot{q}^{2}, \dot{q}^{3}, \dot{q}^{4}\right)=\left(q^{2}+q^{3}\right) \dot{q}^{1}+q^{4} \dot{q}^{3}+\frac{1}{2}\left(\left(q^{4}\right)^{2}-2 q^{2} q^{3}-\left(q^{3}\right)^{2}\right) .
$$

Then $F L$ is given by $F L: T \mathbb{R}^{4} \rightarrow T^{*} \mathbb{R}^{4}$

$$
F L\left(q^{1}, q^{2}, q^{3}, q^{4}, \dot{q}^{1}, \dot{q}^{2}, \dot{q}^{3}, \dot{q}^{4}\right)=\left(q^{1}, q^{2}, q^{3}, q^{4}, q^{2}+q^{3}, 0, q^{4}, 0\right)
$$

and the primary constraints are

$$
\begin{array}{ll}
\Phi^{1}\left(q^{A}, p_{A}\right)=p_{1}-q^{2}-q^{3}, \Phi^{2}\left(q^{A}, p_{A}\right)=p_{2}, \\
\Phi^{3}\left(q^{A}, p_{A}\right)=p_{3}-q^{4}, \quad \Phi^{4}\left(q^{A}, p_{A}\right)=p_{4} .
\end{array}
$$

So

$$
\begin{array}{r}
M_{1}=\left\{\left(q^{1}, q^{2}, q^{3}, q^{4}, p_{1}, p_{2}, p_{3}, p_{4}\right) \in \mathbb{R}^{8}\right. \text { such that } \\
\left.p_{1}=q^{2}+q^{3}, p_{2}=0, p_{3}=q^{4}, p_{4}=0\right\}
\end{array}
$$

and we can use $\left(q^{1}, q^{2}, q^{3}, q^{4}\right)$ as coordinates on $M_{1}$.

It follows that

$$
\begin{aligned}
& E_{L}=\left(q^{2}+q^{3}\right) \dot{q}^{1}+q^{4} \dot{q}^{3}-L=-\frac{1}{2}\left(\left(q^{4}\right)^{2}-2 q^{2} q^{3}-\left(q^{3}\right)^{2}\right), \\
& h_{1}=-\frac{1}{2}\left(\left(q^{4}\right)^{2}-2 q^{2} q^{3}-\left(q^{3}\right)^{2}\right), \\
& \omega_{1}=d q^{1} \wedge d q^{2}+d q^{1} \wedge d q^{3}+d q^{3} \wedge d q^{4}, \\
& \operatorname{ker}\left(\omega_{1}\right)=\{0\},
\end{aligned}
$$

so $\left(M_{1}, \omega_{1}\right)$ is a symplectic manifold.

If we prefer to follow the Dirac-Bergmann algorithm, then one should take an extension $h\left(q^{A}, p_{A}\right)=-\frac{1}{2}\left(\left(q^{4}\right)^{2}-2 q^{2} q^{3}-\left(q^{3}\right)^{2}\right)$ of $h_{1}$. It is easy to see that at the points of $M_{1}:=\operatorname{Im}(F L)$

$$
\begin{aligned}
& \left\{\Phi^{1}, h+u^{1} \Phi^{1}+u^{2} \Phi^{2}+u^{3} \Phi^{3}+u^{4} \Phi^{4}\right\}=-u^{2}-u^{3}, \\
& \left\{\Phi^{2}, h+u^{1} \Phi^{1}+u^{2} \Phi^{2}+u^{3} \Phi^{3}+u^{4} \Phi^{4}\right\}=-q^{3}+u^{1}, \\
& \left\{\Phi^{3}, h+u^{1} \Phi^{1}+u^{2} \Phi^{2}+u^{3} \Phi^{3}+u^{4} \Phi^{4}\right\}=-q^{2}-q^{3}+u^{1}-u^{4}, \\
& \left\{\Phi^{4}, h+u^{1} \Phi^{1}+u^{2} \Phi^{2}+u^{3} \Phi^{3}+u^{4} \Phi^{4}\right\}=u^{3}+u^{4},
\end{aligned}
$$

which determine completely the Lagrange multipliers:

$$
u^{1}=q^{3}, u^{2}=q^{4}, u^{3}=-q^{4}, u^{4}=-q^{2},
$$

and then all the constraints are of second class.

The solution of the equation $\left(i_{X} \omega_{1}=d h_{1}\right)_{\mid M_{1}}$ is given by

$$
X=q^{3} \frac{\partial}{\partial q^{1}}+q^{4} \frac{\partial}{\partial q^{2}}-q^{4} \frac{\partial}{\partial q^{3}}+\left(2 q^{3}-q^{2}\right) \frac{\partial}{\partial q^{4}}-q^{2} \frac{\partial}{\partial p_{3}}
$$

We will study now the solutions of the Hamilton-Jacobi equation. So, we look for $\gamma \in \Lambda^{1}\left(\mathbb{R}^{4}\right)$ such that 
(i) $\quad \gamma\left(\mathbb{R}^{4}\right) \subset M_{1}$

(ii) $d\left(h_{1} \circ \gamma\right)=0$

(iii) $d \gamma=0$

If $\gamma\left(q^{A}\right)=\left(q^{A}, \gamma_{1}\left(q^{A}\right), \gamma_{2}\left(q^{A}\right), \gamma_{3}\left(q^{A}\right), \gamma_{4}\left(q^{A}\right)\right)$, then the condition $\gamma(Q) \subset M_{1}$ gives

$$
\begin{aligned}
& \gamma_{1}\left(q^{1}, q^{2}, q^{3}, q^{4}\right)=q^{2}+q^{3}, \\
& \gamma_{2}\left(q^{1}, q^{2}, q^{3}, q^{4}\right)=0, \\
& \gamma_{3}\left(q^{1}, q^{2}, q^{3}, q^{4}\right)=q^{4}, \\
& \gamma_{4}\left(q^{1}, q^{2}, q^{3}, q^{4}\right)=0 .
\end{aligned}
$$

But $h_{1} \circ \gamma=-\frac{1}{2}\left(\left(q^{4}\right)^{2}-2 q^{2} q^{3}-\left(q^{3}\right)^{2}\right)$, so the equation $d\left(h_{1} \circ \gamma\right)=0$ holds if and only if $-\frac{1}{2}\left(\left(q^{4}\right)^{2}-2 q^{2} q^{3}-\left(q^{3}\right)^{2}\right)=$ constant, and so there is no solution for the Hamilton-Jacobi equation.

Example 4.12. This example has been discussed by Sundermeyer. ${ }^{41}$ It is an example where only primary first class constraints appear. Let $L$ be the Lagrangian $L: T \mathbb{R}^{2} \rightarrow \mathbb{R}$ given by

$$
L\left(q^{1}, q^{2}, \dot{q}^{1}, \dot{q}^{2}\right)=\frac{1}{2}\left(\dot{q}^{1}\right)^{2}+\dot{q}^{2} q^{1}+\dot{q}^{1} q^{2} .
$$

Then $F L$ is given by $F L: T \mathbb{R}^{4} \rightarrow T^{*} \mathbb{R}^{4}$

$$
F L\left(q^{1}, q^{2}, \dot{q}^{1}, \dot{q}^{2}\right)=\left(q^{1}, q^{2}, \dot{q}^{1}+q^{2}, q^{1}\right)
$$

and the primary constraints are

$$
\Phi^{1}\left(q^{A}, p_{A}\right)=p_{2}-q^{1}
$$

So

$$
M_{1}=\left\{\left(q^{1}, q^{2}, p_{1}, p_{2}\right) \in \mathbb{R}^{4} \text { such that } p_{2}=q^{1}\right\},
$$

and we can use $\left(q^{1}, q^{2}, p_{1}\right)$ as coordinates on $M_{1}$.

It follows that

$$
\begin{aligned}
& E_{L}=\frac{1}{2}\left(\dot{q}^{1}\right)^{2} \\
& h_{1}=\frac{1}{2}\left(p_{1}-q^{2}\right)^{2} \\
& \omega_{1}=d q^{1} \wedge d p_{1}+d q^{2} \wedge d q^{1} \\
& \operatorname{ker}\left(\omega_{1}\right)=\left\langle\frac{\partial}{\partial p_{1}}-\frac{\partial}{\partial q^{2}}\right\rangle
\end{aligned}
$$

Let

$$
h\left(q^{A}, p_{A}\right)=\frac{1}{2}\left(p_{1}-q^{2}\right)^{2}
$$

be an extension of the hamiltonian.

It is easy to see that at the points of $M_{1}:=\operatorname{Im}(F L)$

$$
\left\{\Phi^{1}, h+u \Phi^{1}\right\}=0
$$

and therefore we have global dynamics.

The solution of the equation $\left(i_{X} \omega_{1}=d h_{1}\right)_{\mid M_{1}}$ is given by

$$
X=\left(p_{1}-q^{2}\right) \frac{\partial}{\partial q^{1}}+f \frac{\partial}{\partial q^{2}}+f \frac{\partial}{\partial p_{1}}+\left(p_{1}-q^{2}\right) \frac{\partial}{\partial p_{2}},
$$

where $f \in C^{\infty}\left(M_{1}\right)$

If we now look for $\gamma \in \Lambda^{1}\left(\mathbb{R}^{4}\right)$ such that

(i) $\quad \gamma\left(\mathbb{R}^{2}\right) \subset M_{1}$,

(ii) $d\left(h_{1} \circ \gamma\right)=0$, 
(iii) $d \gamma=0$,

then $\gamma\left(q^{1}, q^{2}\right)=\left(q^{1}, q^{2}, \gamma_{1}\left(q^{1}, q^{2}\right), \gamma_{2}\left(q^{1}, q^{2}\right)\right)$ given by

$$
\gamma\left(q^{1}, q^{2}\right)=\left(q^{1}, q^{2}, q^{2}, q^{1}\right)
$$

satisfies all the required conditions, because $p_{1}\left(\gamma\left(q^{1}, q^{2}\right)\right)=q^{2}, \gamma=d\left(q^{1} \cdot q^{2}\right)$ and $h_{1} \circ \gamma\left(q^{1}, q^{2}\right)$ $=\frac{1}{2}\left(q^{2}-q^{2}\right)=0$. Given an arbitrary solution $X=\left(p_{1}-q^{2}\right) \frac{\partial}{\partial q^{1}}+f \frac{\partial}{\partial q^{2}}+f \frac{\partial}{\partial p_{1}}+\left(p_{1}-q^{2}\right) \frac{\partial}{\partial p_{2}}$ of the constrained dynamics, we have that

$$
X^{\gamma}=(f \circ \gamma) \frac{\partial}{\partial q^{2}}
$$

and also

$$
T \gamma\left(X^{\gamma}\right)=(f \circ \gamma) \frac{\partial}{\partial q^{2}}+(f \circ \gamma) \frac{\partial}{\partial p^{1}}
$$

which is precisely $X$ along $\operatorname{Im}(\gamma)$.

\section{There are secondary constraints}

Next, we are going to describe several examples where secondary constraints appear.

Example 4.13. This example has been discussed by Gotay and Nester. ${ }^{19}$ It is an example where first and second class secondary constraints appear. Let $L$ be the Lagrangian $L: T \mathbb{R}^{2} \rightarrow \mathbb{R}$ given by

$$
L\left(q^{1}, q^{2}, \dot{q}^{1}, \dot{q}^{2}\right)=\frac{1}{2}\left(\dot{q}^{1}\right)^{2}+q^{2}\left(q^{1}\right)^{2} .
$$

Then $F L$ is given by $F L: T \mathbb{R}^{2} \rightarrow T^{*} \mathbb{R}^{2}$

$$
F L\left(q^{1}, q^{2}, \dot{q}^{1}, \dot{q}^{2}\right)=\left(q^{1}, q^{2}, \dot{q}^{1}, 0\right)
$$

and the primary constraints are

$$
\Phi^{1}\left(q^{A}, p_{A}\right)=p_{2}
$$

So

$$
M_{1}=\left\{\left(q^{1}, q^{2}, p_{1}, p_{2}\right) \in \mathbb{R}^{4} \text { such that } p_{2}=0\right\}
$$

and we can use $\left(q^{1}, q^{2}, p_{1}\right)$ as coordinates on $M_{1}$.

It follows that

$$
\begin{aligned}
& E_{L}=\frac{1}{2}\left(\dot{q}^{1}\right)^{2}-q^{2}\left(q^{1}\right)^{2}, \\
& h_{1}=\frac{1}{2}\left(p_{1}\right)^{2}-q^{2}\left(q^{1}\right)^{2}, \\
& \omega_{1}=d q^{1} \wedge d p_{1} \\
& \operatorname{ker}\left(\omega_{1}\right)=\left\langle\frac{\partial}{\partial q^{2}}\right\rangle
\end{aligned}
$$

Let

$$
h\left(q^{A}, p_{A}\right)=\frac{1}{2}\left(p_{1}\right)^{2}-q^{2}\left(q^{1}\right)^{2}
$$

be an arbitrary extension of the constrained hamiltonian $h_{1}$ to $T^{*} \mathbb{R}^{2}$.

It is easy to see that at the points of $M_{1}:=\operatorname{Im}(F L)$ we have

$$
\left\{\Phi^{1}, h+u^{1} \Phi^{1}\right\}=-\left(q^{1}\right)^{2}
$$

and therefore we need to restrict the dynamics adding a new constraint

$$
\Phi^{2}\left(q^{A}, p_{A}\right)=q^{1} .
$$


Now $\quad M_{2}:=\left\{\left(q^{1}, q^{2}, p_{1}, p_{2}\right) \in \mathbb{R}^{4}\right.$ such that $\left.p_{2}=0, \quad q^{1}=0\right\} \quad$ and $\quad Q_{2}:=\pi_{Q}\left(M_{2}\right)=\left\{\left(q^{1}, q^{2}\right)\right.$ $\in \mathbb{R}^{2}$ such that $\left.q^{1}=0\right\}$. We have on $M_{2}$

$$
\begin{aligned}
& \left\{\Phi^{1}, h+u^{1} \Phi^{1}\right\}=0, \\
& \left\{\Phi^{2}, h+u^{1} \Phi^{1}\right\}=p_{1},
\end{aligned}
$$

and we need to restrict again the dynamics, adding the constraint

$$
\Phi^{3}\left(q^{A}, p_{A}\right)=p_{1}
$$

Now $\quad M_{3}:=\left\{\left(q^{1}, q^{2}, p_{1}, p_{2}\right) \in \mathbb{R}^{4}\right.$ such that $\left.p_{2}=0, \quad q^{1}=0, \quad p_{1}=0\right\} \quad$ and $\quad Q_{3}=Q_{2}$ $=\left\{\left(q^{1}, q^{2}\right) \in \mathbb{R}^{2}\right.$ such that $\left.q^{1}=0\right\}$. Along $M_{3}$ we have

$$
\begin{aligned}
& \left\{\Phi^{1}, h+u^{1} \Phi^{1}\right\}=0, \\
& \left\{\Phi^{2}, h+u^{1} \Phi^{1}\right\}=0, \\
& \left\{\Phi^{3}, h+u^{1} \Phi^{1}\right\}=0,
\end{aligned}
$$

and $M_{3}$ is the final constraint submanifold $M_{f}$. We can easily check that $\Phi^{1}$ is a first class constraint and $\Phi^{2}, \Phi^{3}$ are second class.

The solutions of the equation $\left(i_{X} \omega_{1}=d h_{1}\right)_{\mid M_{3}}$ are of the form

$$
X=f \frac{\partial}{\partial q^{2}}
$$

where $f \in C^{\infty}\left(M_{3}\right)$.

A solution of the Hamilton-Jacobi equation, should be $\gamma\left(q^{1}, q^{2}\right)=\left(q^{1}, q^{2}, \gamma_{1}\left(q^{1}, q^{2}\right), \gamma_{2}\left(q^{1}\right.\right.$, $\left.q^{2}\right)$ ), such that

(i) $\quad \gamma(Q) \subset M_{1}$ and $\gamma_{f}\left(Q_{f}\right) \subset M_{f}$,

(ii) $d\left(h_{1} \circ \gamma\right)_{\mid Q_{f}}=0$,

(iii) $d \gamma=0$.

The condition $\gamma(Q) \subset M_{1}$ implies $\gamma_{2}=0$. Next we compute $d \gamma$,

$$
d \gamma=\frac{\partial \gamma_{1}}{\partial q^{2}} d q^{2} \wedge d q^{1}+\frac{\partial \gamma_{2}}{\partial q^{1}} d q^{1} \wedge d q^{2}=\frac{\partial \gamma_{1}}{\partial q^{2}} d q^{2} \wedge d q^{1}=0
$$

and we deduce that $\frac{\partial \gamma_{1}}{\partial q^{2}}$ must vanish and $\gamma_{1}$ must be a function of $q^{1}$.

The condition $d\left(h_{1} \circ \gamma\right)_{\mid Q_{f}}=0$ can also be easily computed. We have

$$
d\left(h_{1} \circ \gamma\right)=d\left(\frac{1}{2}\left(\gamma_{1}\right)^{2}-q^{2}\left(q^{1}\right)^{2}\right)=\left(\gamma_{1} \frac{\partial \gamma_{1}}{\partial q^{1}}-2 q^{2} q^{1}\right) d q^{1}-\left(q^{1}\right)^{2} d q^{2}
$$

and, along $Q_{f}=\left\{\left(q^{1}, q^{2}\right) \in \mathbb{R}^{2}\right.$ such that $\left.q^{1}=0\right\}$, we deduce

$$
d\left(h_{1} \circ \gamma\right)_{\mid Q_{f}}=\gamma_{1}\left(0, q^{2}\right) \frac{\partial \gamma_{1}}{\partial q^{1}}\left(0, q^{2}\right) d q^{1} .
$$
$M_{f}$.

For example, if we take $\gamma_{1}=q^{1}, \gamma_{2}=0$, all the above conditions are satisfied, and $\gamma_{f}\left(Q_{f}\right) \subset$

Now, take a solution $X=f \frac{\partial}{\partial q^{2}}$; at the points of $Q_{f}$ we get

$$
X^{\gamma}\left(0, q^{2}\right)=\left(\pi_{f}\right)_{*}\left(f\left(0, q^{2}, 0,0\right) \frac{\partial}{\partial q^{2}}\right)=f\left(0, q^{2}, 0,0\right) \frac{\partial}{\partial q^{2}}
$$

and so

$$
T \gamma_{f}\left(X^{\gamma}\left(0, q^{2}\right)\right)=f\left(0, q^{2}, 0,0\right) \frac{\partial}{\partial q^{2}},
$$

and we obtain the solution $X$ along $\operatorname{Im}\left(\gamma_{f}\right)$. 
Example 4.14. This example has been discussed by Gotay. ${ }^{15}$ It is an example where secondary constraints appear and all constraints are first class. Let $Q:=\left\{\left(q^{1}, q^{2}\right) \in \mathbb{R}^{2}\right.$ such that $\left.q^{1} \neq 0\right\}$ and $L$ be the Lagrangian $L: T Q \rightarrow \mathbb{R}$ given by

$$
L\left(q^{1}, q^{2}, \dot{q}^{1}, \dot{q}^{2}\right)=\frac{1}{2 q^{1}}\left(\dot{q}^{2}\right)^{2} .
$$

Then $F L$ is given by $F L: T Q \rightarrow T^{*} Q$

$$
F L\left(q^{1}, q^{2}, \dot{q}^{1}, \dot{q}^{2}\right)=\left(q^{1}, q^{2}, 0, \dot{q}^{2} / q^{1}\right)
$$

and the primary constraints are

$$
\Phi^{1}\left(q^{A}, p_{A}\right)=p_{1}
$$

So

$$
M_{1}=\left\{\left(q^{1}, q^{2}, p_{1}, p_{2}\right) \in T^{*} Q \text { such that } p_{1}=0\right\} .
$$

and we can use $\left(q^{1}, q^{2}, p_{2}\right)$ as coordinates on $M_{1}$.

It follows that

$$
\begin{aligned}
& E_{L}=L, \\
& h_{1}\left(q^{A}, p_{A}\right)=\frac{q^{1}}{2}\left(p_{2}\right)^{2}, \\
& \omega_{1}=d q^{2} \wedge d p_{2}, \\
& \operatorname{ker}\left(\omega_{1}\right)=\left\langle\frac{\partial}{\partial q^{1}}\right\rangle .
\end{aligned}
$$

Let $h\left(q^{A}, p_{A}\right)=\frac{q^{1}}{2}\left(p_{2}\right)^{2}$ be an extension of the hamiltonian.

It is easy to see that at the points of $M_{1}:=\operatorname{Im}(F L)$ we get

$$
\left\{\Phi^{1}, h+u^{1} \Phi^{1}\right\}=-\frac{\left(p_{2}\right)^{2}}{2}
$$

and therefore we need to restrict the dynamics adding a new constraint

$$
\Phi^{2}\left(q^{A}, p_{A}\right)=p_{2} .
$$

Now $M_{2}:=\left\{\left(q^{1}, q^{2}, p_{1}, p_{2}\right) \in T Q\right.$ such that $\left.p_{1}=0, p_{2}=0\right\}$ and $Q_{2}:=\pi_{Q}\left(M_{2}\right)=Q$. At the points of $M_{2}$ we have

$$
\begin{aligned}
& \left\{\Phi^{1}, h+u^{1} \Phi^{1}\right\}=0, \\
& \left\{\Phi^{2}, h+u^{1} \Phi^{1}\right\}=0,
\end{aligned}
$$

and $M_{2}$ is the final constraint manifold. From $\left\{\Phi^{1}, \Phi^{2}\right\}=0$ we deduce that the constraints are all first class.

The solutions are of the form $X=f \frac{\partial}{\partial q^{1}}$ where $f \in C^{\infty}\left(M_{2}\right)$.

If we look for a solution of our Hamilton-Jacobi equation, $\gamma$, such that $\gamma\left(q^{1}, q^{2}\right)=\left(q^{1}, q^{2}\right.$, $\left.\gamma_{1}\left(q^{1}, q^{2}\right), \gamma_{2}\left(q^{1}, q^{2}\right)\right)$, then the condition $\gamma(Q) \subset M_{1}$ implies $\gamma=0$ and $\gamma_{f}=0$. All conditions are verified and, given a solution $X$, we obtain that $X^{\gamma}$ and $X$ are trivially $\gamma_{f}$-related.

Example 4.15. This example has been discussed by Skinner and Rusk. ${ }^{39}$ It is an example where secondary constraints appear and all constraints are first class. Let $L$ be the Lagrangian $L: T \mathbb{R}^{3} \rightarrow \mathbb{R}$ given by

$$
L\left(q^{1}, q^{2}, q^{3}, \dot{q}^{1}, \dot{q}^{2}, \dot{q}^{3}\right)=\frac{1}{2} q^{2}\left(q^{3}\right)^{2}+\dot{q}^{1} \dot{q}^{3} .
$$

Then $F L: T \mathbb{R}^{3} \rightarrow T^{*} \mathbb{R}^{3}$ is given by

$$
F L\left(q^{1}, q^{2}, q^{3}, \dot{q}^{1}, \dot{q}^{2}, \dot{q}^{3}\right)=\left(q^{1}, q^{2}, q^{3}, \dot{q}^{3}, 0, \dot{q}^{1}\right),
$$


so that we have a primary constraint $\Phi^{1}\left(q^{A}, p_{A}\right)=p_{2}$. This means that the primary constraint submanifold is

$$
M_{1}=\left\{\left(q^{1}, q^{2}, q^{3}, p_{1}, p_{2}, p_{3}\right) \in \mathbb{R}^{6} \text { such that } p_{2}=0\right\},
$$

and then we can use $\left(q^{1}, q^{2}, q^{3}, p_{1}, p_{3}\right)$ as coordinates on $M_{1}$.

It follows that

$$
\begin{aligned}
& E_{L}=\dot{q}^{3} \dot{q}^{1}+\dot{q}^{1} \dot{q}^{3}-L=-\frac{1}{2} q^{2}\left(q^{3}\right)^{2}+\dot{q}^{1} \dot{q}^{3}, \\
& h_{1}\left(q^{A}, p_{A}\right)=p_{1} p_{3}-\frac{1}{2} q^{2}\left(q^{3}\right)^{2}, \\
& \omega_{1}=d q^{1} \wedge d p_{1}+d q^{3} \wedge d p_{3}, \\
& \operatorname{ker}\left(\omega_{1}\right)=\left\langle\frac{\partial}{\partial q^{2}}\right\rangle .
\end{aligned}
$$

As in the previous cases, take an arbitrary extension of the hamiltonian $h_{1}$, for instance,

$$
h\left(q^{A}, p_{A}\right)=p_{1} p_{3}-\frac{1}{2} q^{2}\left(q^{3}\right)^{2} .
$$

It is easy to see that at the points of $M_{1}:=\operatorname{Im}(F L)$

$$
\left\{\Phi^{1}, h+u^{1} \Phi^{1}\right\}=\frac{1}{2}\left(q^{3}\right)^{2}
$$

and therefore we should restrict the dynamics adding a secondary constraint

$$
\Phi^{2}\left(q^{A}, p_{A}\right)=q^{3} .
$$

Now $\quad M_{2}=\left\{\left(q^{1}, q^{2}, q^{3}, p_{1}, p_{2}, p_{3}\right) \in \mathbb{R}^{6}\right.$ such that $\left.p_{2}=0, \quad q^{3}=0\right\}, \quad$ and $\quad Q_{2}:=\pi_{Q}\left(M_{2}\right)$ $=\left\{\left(q^{1}, q^{2}, q^{3}\right) \in \mathbb{R}^{3}\right.$ such that $\left.q^{3}=0\right\}$. Along $M_{2}$, we have

$$
\begin{aligned}
& \left\{\Phi^{1}, h+u^{1} \Phi^{1}\right\}=0, \\
& \left\{\Phi^{2}, h+u^{1} \Phi^{1}\right\}=p_{1} .
\end{aligned}
$$

Therefore, we need again to restrict the dynamics, adding the constraint $\Phi^{3}\left(q^{A}, p_{A}\right)$ $=p_{1}$. Now $M_{3}:=\left\{\left(q^{1}, q^{2}, q^{3}, p_{1}, p_{2}, p_{3}\right) \in \mathbb{R}^{6}\right.$ such that $\left.p_{2}=0, q^{3}=0, \quad p_{1}=0\right\}$ and $Q_{3}$ $=Q_{2}=\left\{\left(q^{1}, q^{2}, q^{3}\right) \in \mathbb{R}^{3}\right.$ such that $\left.q^{3}=0\right\}$. Along $M_{3}$ we have

$$
\begin{aligned}
& \left\{\Phi^{1}, h+u^{1} \Phi^{1}\right\}=0, \\
& \left\{\Phi^{2}, h+u^{1} \Phi^{1}\right\}=0, \\
& \left\{\Phi^{3}, h+u^{1} \Phi^{1}\right\}=0,
\end{aligned}
$$

and then $M_{3}$ is the final constraint manifold, denoted by $M_{f}$; therefore, $Q_{f}=Q_{3}$. We deduce that the constraints are all first class.

The solutions of the equation $\left(i_{X} \omega_{1}=d h_{1}\right)_{\mid M_{3}}$ are of the form

$$
X=p_{3} \frac{\partial}{\partial q^{1}}+f \frac{\partial}{\partial q^{2}},
$$

where $f \in C^{\infty}\left(M_{3}\right)$.

Now we look for a solution of our Hamilton-Jacobi equation, that is $\gamma\left(q^{1}, q^{2}, q^{3}\right)=\left(q^{1}, q^{2}, q^{3}\right.$, $\left.\gamma_{1}\left(q^{1}, q^{2}, q^{3}\right), \gamma_{2}\left(q^{1}, q^{2}, q^{3}\right), \gamma_{3}\left(q^{1}, q^{2}, q^{3}\right)\right)$, such that

(i) $\quad \gamma(Q) \subset M_{1}$ and $\gamma_{f}\left(Q_{f}\right) \subset M_{f}$,

(ii) $d\left(h_{1} \circ \gamma\right)_{\mid Q_{f}}=0$,

(iii) $d \gamma=0$. 
The condition $\gamma(Q) \subset M_{1}$ implies $\gamma_{2}=0$; the condition $\gamma_{f}\left(Q_{f}\right) \subset M_{f}$ implies $\left(\gamma_{f}\right)_{i}=0$ for $i=$ 1,2 and, the condition $d\left(h_{1} \circ \gamma\right)_{Q_{f}}=0$ is

$$
\begin{aligned}
& d\left(h_{1} \circ \gamma\right)=d\left(\gamma_{1} \gamma_{3}-\frac{1}{2} q^{2}\left(q^{3}\right)^{2}\right) \\
& =\left(\frac{\partial \gamma_{1}}{\partial q^{1}} \gamma_{3}+\frac{\partial \gamma_{3}}{\partial q^{1}} \gamma_{1}\right) d q^{1}+\left(\frac{\partial \gamma_{1}}{\partial q^{2}} \gamma_{3}+\frac{\partial \gamma_{3}}{\partial q^{2}} \gamma_{1}-\frac{1}{2}\left(q^{3}\right)^{2}\right) d q^{2} \\
& +\left(\frac{\partial \gamma_{1}}{\partial q^{3}} \gamma_{3}+\frac{\partial \gamma_{3}}{\partial q^{3}} \gamma_{1}-q^{2} q^{3}\right) d q^{3}
\end{aligned}
$$

Hence,

$$
\begin{aligned}
& d\left(h_{1} \circ \gamma\right)_{\mid Q_{f}}=\left(\frac{\partial \gamma_{1}}{\partial q^{1}} \gamma_{3}+\frac{\partial \gamma_{3}}{\partial q^{1}} \gamma_{1}\right) d q^{1}+\left(\frac{\partial \gamma_{1}}{\partial q^{2}} \gamma_{3}+\frac{\partial \gamma_{3}}{\partial q^{2}} \gamma_{1}\right) d q^{2} \\
& +\left(\frac{\partial \gamma_{1}}{\partial q^{3}} \gamma_{3}+\frac{\partial \gamma_{3}}{\partial q^{3}} \gamma_{1}\right) d q^{3}
\end{aligned}
$$

The condition $d \gamma=0$ implies

$$
\begin{aligned}
d \gamma= & \frac{\partial \gamma_{1}}{\partial q^{2}} d q^{2} \wedge d q^{1}+\frac{\partial \gamma_{1}}{\partial q^{3}} d q^{3} \wedge d q^{1} \\
& +\frac{\partial \gamma_{3}}{\partial q^{1}} d q^{1} \wedge d q^{3}+\frac{\partial \gamma_{3}}{\partial q^{2}} d q^{2} \wedge d q^{3}=0
\end{aligned}
$$

taking into account that $\gamma_{2}=0$, and therefore

$$
\begin{aligned}
& \frac{\partial \gamma_{1}}{\partial q^{2}}=0, \\
& \frac{\partial \gamma_{1}}{\partial q^{3}}=\frac{\partial \gamma_{3}}{\partial q^{1}}, \\
& \frac{\partial \gamma_{3}}{\partial q^{2}}=0 .
\end{aligned}
$$

A particular solution is obtained putting $\gamma_{1}=\gamma_{2}=0$, and $\gamma_{3}$ an arbitrary function of $q^{3}$, for example $\gamma_{3}=q^{3}$.

For instance, take $X=p_{3} \frac{\partial}{\partial q^{1}}+f \frac{\partial}{\partial q^{2}}$, then

$$
\gamma\left(q^{1}, q^{2}, q^{3}\right)=\left(q^{1}, q^{2}, q^{3}, 0,0, q^{3}\right)
$$

and at the points of $Q_{f}$ we obtain

$$
X^{\gamma}\left(q^{1}, q^{2}, 0\right)=T \pi_{f}\left(0 \frac{\partial}{\partial q^{1}}+f\left(q^{1}, q^{2}, 0,0,0,0\right) \frac{\partial}{\partial q^{2}}\right)=f\left(q^{1}, q^{2}, 0,0,0,0\right) \frac{\partial}{\partial q^{2}},
$$

so that

$$
T \gamma_{f}\left(X^{\gamma}\left(q^{1}, q^{2}, 0\right)\right)=f\left(q^{1}, q^{2}, 0,0,0,0\right) \frac{\partial}{\partial q^{2}} .
$$

We can also apply Proposition 4.7 to the latter example and obtain solutions of the extended equation.

For instance, consider $\gamma\left(q^{1}, q^{2}, q^{3}\right)=\left(q^{1}, q^{2}, q^{3}, \gamma_{1}\left(q^{A}\right), \gamma_{2}\left(q^{A}\right), \gamma_{3}\left(q^{A}\right)\right)$ given by

$$
\gamma\left(q^{1}, q^{2}, q^{3}\right)=\left(q^{1}, q^{2}, q^{3}, q^{3}, 0, q^{1}\right) .
$$

We have

(i) $\quad \gamma_{f}\left(Q_{f}\right) \subset M_{f}$ because

$$
\gamma_{f}\left(Q_{f}\right)=\left\{\left(q^{1}, q^{2}, 0,0,0, q^{1}\right) \in \mathbb{R}^{6} \text { such that } q^{1}, q^{2} \in \mathbb{R}\right\} .
$$

(ii) If we take coordinates $\left(q^{1}, q^{2}\right)$ in $Q_{f}$, then $d\left(h_{1} \circ \gamma_{f}\right)=d\left(0 \cdot 0-q^{2} \cdot 0\right)=0$.

(iii) $d \gamma=0$, in fact, $\gamma=d\left(q^{1} \cdot q^{3}\right)$.

If we consider a solution $X=p_{3} \frac{\partial}{\partial q^{1}}+f \frac{\partial}{\partial q^{2}}$, we can compute

$$
X^{\gamma}\left(q^{1}, q^{2}, 0\right)=T \tau_{Q} \circ X \circ \gamma_{f}\left(q^{1}, q^{2}, 0\right)=q^{1} \frac{\partial}{\partial q^{1}}+f\left(q^{1}, q^{2}, 0\right) \frac{\partial}{\partial q^{2}}
$$


and also

$$
T \gamma_{f}\left(X^{\gamma}\left(q^{1}, q^{2}, 0\right)\right)=q^{1} \frac{\partial}{\partial q^{1}}+f\left(q^{1}, q^{2}, 0\right) \frac{\partial}{\partial q^{2}}+q^{1} \frac{\partial}{\partial p_{3}},
$$

which is a solution of the equation $i_{X} \omega_{3}=d h_{3}$ where, if $i_{3}: M_{3} \rightarrow T^{*} Q$ is the inclusion on $T^{*} Q$ and $j_{3}: M_{3} \rightarrow M_{1}$, then $\omega_{3}=i_{3}^{*}\left(\omega_{Q}\right)$ and $h_{3}=j_{3}^{*}\left(h_{1}\right)$.

Note that $\gamma$ in this case is not a solution of our Hamilton-Jacobi problem because $d(h \circ \gamma)\left(q^{1}\right.$, $\left.q^{2}, 0\right)=q^{1} d q^{3} \neq 0$.

\section{F. Relation to classical Hamilton-Jacobi theory}

In this section we will connect the Hamilton-Jacobi theory developed in Secs. I- IV with the classical Hamilton-Jacobi theory on $T^{*} Q$ using an appropriate extended hamiltonian.

We will use the same notation that is given in Sec. IVD. We start with an almost regular lagrangian $L: T Q \rightarrow \mathbb{R}$, and then $\operatorname{Im}(F L)=M_{1}$ is a differentiable submanifold of $T^{*} Q$ and, in addition, we can define $h_{1}$ implicitly by $h_{1} \circ F L=E_{L}$. We denote $\omega_{1}=j_{1}^{*} \omega_{Q}$, where $j_{1}: M_{1} \rightarrow$ $T^{*} Q$ is the inclusion and $\omega_{Q}$ is the canonical symplectic form of the cotangent bundle. We take local coordinates $\left(q^{A}, p_{A}\right)$ in an open set $U \subset T^{*} Q$, such that $M_{1}$ is given locally by the vanishing of independent functions $\Phi^{i}\left(q^{A}, p_{A}\right)$, called primary constraints.

Remember that the equations of motion have the form $\left(i_{X} \omega_{1}=d h_{1}\right)_{\mid U_{1}}$, where $U_{1}:=M_{1} \cap$ $U$. These equations are equivalent to $\left(i_{X} \omega_{Q}=d h+\mu_{i} d \Phi^{i}\right)_{\mid U_{1}}$ where $h$ is any extension of $h_{1}$ to $U$ defined on $M_{1}$ and $\mu_{i}$ are Lagrange multipliers.

\section{Case I: There are only primary constraints}

First, we suppose that there exist a global solution $X$, i.e., $X$ is a vector field on $M_{1}$ that satisfies the equations of motion. We reorder constraint functions in two classes: first class constraints denoted by $\chi^{a}$ and second class constraints denoted by $\xi^{b}$. We also denote by $u_{a}$ and $\lambda_{b}$ the corresponding Lagrange multipliers. Then the equations of motion are

$$
\left(i_{X} \omega_{Q}=d h+u_{a} d \chi^{a}+\lambda_{b} d \xi^{b}\right)_{\mid M_{1}} .
$$

Now, suppose that $u_{a}$ and $\lambda_{b}$ are functions defined on $U$. It is clear that $X$ is the restriction to $M_{1}$ of the hamiltonian vector field corresponding to a hamiltonian of the form $h+u_{a} \chi^{a}+\lambda_{b} \xi^{b}$. In fact, all the solutions of the equations of motion are obtained in this way varying the functions $u_{a}$ arbitrarily and with prescribed values of $\lambda_{b}$. Next, we are looking for a solution of our Hamilton-Jacobi problem, that is, a 1-form $\gamma$ satisfying

(i) $\quad d \gamma=0$,

(ii) $\operatorname{Im}(\gamma) \subset M_{1}$,

(iii) $d\left(h_{1} \circ \gamma\right)=0$.

Condition (iii) can be easily checked that it is equivalent to $d\left(\left(h+u_{a} \chi^{a}+\lambda_{b} \xi^{b}\right) \circ \gamma\right)=0$ because $\left(h+u^{a} \chi^{a}+\lambda^{b} \xi^{b}\right)_{\mid M_{1}}=h_{1}$. So, it is evident that the solutions of the classical Hamilton-Jacobi equation for the hamiltonians $h+u^{a} \chi^{a}+\lambda^{b} \xi^{b}$ (where $u^{a}$ are arbitrary functions and the rest are fixed) inside $M_{1}$ and the solutions for our Hamilton-Jacobi problem coincide.

With this local classification in first and second class constraints, $\chi^{a}, \xi^{b}$, on a neighborhood $U$ such that $M_{1} \cap U$ is the set of zeros of these constraints, it is possible to construct the Dirac bracket associated to this decomposition (see Refs. 21,27, and 40). Observe that in $U$, since the constraints $\left\{\xi^{b}\right\}$ are second class then the matrix:

$$
\left(\mathcal{C}^{b b^{\prime}}\right)=\left(\left\{\xi^{b}, \xi^{b^{\prime}}\right\}\right)
$$

is nonsingular in $U_{1}=M_{1} \cap U$, so it is obviously nonsingular in a neighbourhood $\bar{U}$ of $M_{1} \cap U$; for simplicity we can assume that $\bar{U}=U$. This condition is equivalent to say that the distribution

$$
\mathcal{A}=\operatorname{span}\left\{X_{\xi^{b}}\right\}
$$


is symplectic, that is $\mathcal{A} \cap \mathcal{A}^{\perp}=0$ along $U$. Therefore, we have the following decomposition:

$$
T U=\mathcal{A} \oplus \mathcal{A}^{\perp}
$$

with induced projectors

$$
\mathcal{P}: T U \longrightarrow \mathcal{A}^{\perp}, \quad \mathcal{Q}: T U \longrightarrow \mathcal{A}
$$

Observe that by definition

$$
\mathcal{P}=\operatorname{id}_{T U}-\mathcal{C}_{b b^{\prime}} X_{\xi^{b}} \otimes d \xi^{b^{\prime}},
$$

where $\mathcal{C}_{b b^{\prime}}$ are the components of the inverse matrix of $\left(\mathcal{C}^{b b^{\prime}}\right)$. Define the Dirac bracket by

$$
\{f, g\}_{D}=\omega_{Q}\left(\mathcal{P}\left(X_{f}\right), \mathcal{P}\left(X_{g}\right)=\Lambda_{Q}\left(\mathcal{P}^{*}(d f), \mathcal{P}^{*}(d g)\right)\right.
$$

for arbitrary functions $f, g$ on $U$ and where $\Lambda_{Q}$ denotes the Poisson tensor corresponding to $\omega_{Q}$. From this definition we deduce the classical form of the Dirac bracket

$$
\{f, g\}_{D}=\{f, g\}-\left\{f, \xi^{b}\right\} \mathcal{C}_{b b^{\prime}}\left\{\xi^{b^{\prime}}, g\right\} .
$$

From construction the Dirac bracket verifies the Jacobi identity due to the involutive character of $\mathcal{A}^{\perp}$ is obviously involutive since its annihilator is generated by differentials of a set of second class constraints (see, for instance, Proposition 3.19 in Ref. 5 or Proposition 3.4 in Ref. 21). The construction of the Dirac bracket relies on the choice of a symplectic complement $\mathcal{A}$. Observe that this choice is locally equivalent to select an independent set of second class constraints.

Fixed an arbitrary extension $h: U \rightarrow \mathbb{R}$ of $h_{1}: M_{1} \rightarrow \mathbb{R}$, we have that the evolution equations are given by the formula

$$
\dot{f}=\{f, h\}_{D}, \quad f \in C^{\infty}(U) .
$$

The hamiltonian vector field $X_{h, D}$ corresponding to $h$ for the Dirac bracket is precisely $\mathcal{P}\left(X_{h}\right)$ and we have the following rewriting of Proposition 4.3.

Proposition 4.16. Let $\gamma$ a closed 1-form on $Q$ such that $\gamma(Q) \subseteq M_{1}$, then the following conditions are equivalent:

(i) $X_{h, D}$ and $X_{h, D}^{\gamma}$ are $\gamma$-related,

(ii) $d(h \circ \gamma)=0$,

where $X_{h, D}^{\gamma}=T \pi_{Q} \circ X_{h, D} \circ \gamma$.

The condition $\gamma(Q) \subseteq M_{1}$ appears in Proposition 4.16 as a dynamical assumption since we are only interested in the dynamics restricted to $M_{1}$.

\section{Case II: The general case}

Suppose now that the algorithm do not stop at $M_{1}$, then we obtain the sequence of manifolds

$$
\cdots M_{k} \hookrightarrow \cdots \hookrightarrow M_{2} \hookrightarrow M_{1} \hookrightarrow T^{*} Q .
$$

and we suppose that the algorithm stabilizes in a manifold $M_{f}$ of dimension $>0$.

Take a set of independent constraints determining the submanifold $M_{1}$, that is, a set of primary constraints $\left\{\Phi^{\rho}\right\}$. Therefore in a neighborhood $U, M_{1} \cap U$ is the set of zeros of these constraints. In classical Dirac notation, $M_{1} \cap U$ is defined by the weak equations:

$$
\Phi^{\rho} \approx 0 .
$$

Complete the above set of primary constraints with secondary constraints $\zeta^{\nu}$, such that $M_{f} \cap U$ is defined by

$$
\Phi^{\rho} \approx 0, \quad \varsigma^{\nu} \approx 0
$$

Consider the rectangular matrix

$$
\left(\left\{\Phi^{\rho}, \Phi^{\rho^{\prime}}\right\}\left\{\Phi^{\rho}, \varsigma^{\nu}\right\}\right)
$$


If the rank of the matrix is $k$, we can extract a new set $\left\{\chi^{a}, \xi^{b}\right\}$ of primary constraints taking linear combination of the constraints $\left\{\Phi^{\rho}\right\}$ in such a way that $\left\{\chi^{a}\right\}$ is a set of first class constraints, that is,

$$
\left\{\chi^{a}, \Phi^{\rho^{\prime}}\right\} \approx 0, \quad\left\{\chi^{a}, \varsigma^{\nu}\right\} \approx 0
$$

and $\left\{\xi^{b}\right\}, 1 \leq b \leq k$ are primary second class constraints. In the same way, we can split the secondary constraints $\varsigma^{v}$ in secondary first and second class constraints, $\psi^{c}$ and $\theta^{d}$, respectively (see Refs. 21,27, and 40 for more details ). We will also denote by $u_{a}, \lambda_{b}, v_{c}$, and $w_{d}$ the corresponding Lagrange multipliers to the set of constraints $\left\{\chi^{a}, \xi^{b}, \psi^{c}, \theta^{d}\right\}$. Again a solution $X$ of the equations of motion verifies

$$
\left(i_{X} \omega_{Q}=d h+u_{a} d \chi^{a}+\lambda_{b} d \xi^{b}\right)_{\mid M_{f}} .
$$

As above, $X$ is the restriction to $M_{f}$ of the hamiltonian vector field given by the hamiltonian $h$ $+u_{a} \chi^{a}+\lambda_{b} \xi^{b}$ where some multipliers are determined applying the constraint algorithm.

We are looking for $\gamma \in \Lambda^{1}(Q)$ satisfying

(i) $d \gamma=0$,

(ii) $\operatorname{Im}(\gamma) \subset M_{1}$,

(iii) $\gamma\left(Q_{f}\right) \subset M_{f}$,

(iv) $d\left(h_{1} \circ \gamma_{1}\right)_{\mid Q_{f}}=0$.

Note that (iv) is equivalent to the equation $d\left(\left(h+u_{a} \chi^{a}+\lambda_{b} \xi^{b}\right) \circ \gamma\right)=0$ because $\left(h+u_{a} \chi^{a}\right.$ $\left.+\lambda_{b} \xi^{b}\right)_{\mid M_{1}}=h_{1}$, and so, the solutions $\gamma: Q \rightarrow T^{*} Q$ of the classical Hamilton-Jacobi theory for the hamiltonians $h+u_{a} \chi^{a}+\lambda_{b} \xi^{b}$ such that $\gamma(Q) \subseteq M_{1}$ and $\gamma\left(Q_{f}\right) \subseteq M_{f}$ are just the solutions of our Hamilton-Jacobi problem.

As in Subsection IV F 1, we can use Dirac brackets to describe the dynamics of the constrained system. Denote by $\left\{\Psi^{e}\right\}=\left\{\xi^{b}, \theta^{d}\right\}$ the complete set of second class constraints. Therefore the matrix:

$$
\left(\mathcal{C}^{e e^{\prime}}\right)=\left(\left\{\Psi^{e}, \Psi^{e^{\prime}}\right\}\right)
$$

is nonsingular in $U$ or equivalently the distribution $\mathcal{A}=\operatorname{span}\left\{X_{\Psi^{e}}\right\}$ is symplectic and we have the decomposition $T U=\mathcal{A} \oplus \mathcal{A}^{\perp}$ with induced projectors

$$
\mathcal{P}: T U \longrightarrow \mathcal{A}^{\perp}, \quad \mathcal{Q}: T U \longrightarrow \mathcal{A}
$$

Associated to the projector $\mathcal{P}$ we have the corresponding Dirac bracket $\{,\}_{D}$

$$
\{f, g\}_{D}=\{f, g\}-\left\{f, \Psi^{e}\right\} \mathcal{C}_{e e^{\prime}}\left\{\Psi^{e^{\prime}}, g\right\}
$$

where $\mathcal{C}_{e e^{\prime}}$ are the components of the inverse matrix of $\left(\mathcal{C}^{e e^{\prime}}\right)$. The hamiltonian vector field $X_{h, D}$ for this bracket is precisely $\mathcal{P}\left(X_{h}\right)$. For any extension $h: U \rightarrow \mathbb{R}$ of the hamiltonian $\left(h_{1}\right)_{\mid M_{1} \cap U}$ : $M_{1} \cap U \rightarrow \mathbb{R}$ we have that $\mathcal{P}\left(X_{h}\right)$ is a solution of the equation

$$
\left(i_{X} \omega_{1}=d h_{1}\right)_{\mid M_{f}} .
$$

Finally, we have the following rewriting of Proposition 4.5.

Proposition 4.17. Let $\gamma$ a closed 1-form on $Q$ such that $\gamma(Q) \subseteq M_{1}$ and $\gamma\left(Q_{f}\right) \subseteq M_{f}$, then the following conditions are equivalent:

(i) $X_{h, D}$ and $X_{h, D}^{\gamma}$ are $\gamma_{f}$-related,

(ii) $d(h \circ \gamma)_{\mid Q_{f}}=0$,

where $X_{h, D}^{\gamma}=T \pi_{Q} \circ X_{h, D} \circ \gamma$.

\section{G. Relation to other theories}

The Hamilton-Jacobi theory for degenerate lagrangians have been discussed by several authors in the last 20 years. Let us recall some previous attempts. 
(i) In the papers by Longhi et al. ${ }^{31,32}$ it is discussed the case of a time independent lagrangian which is homogeneous in the velocities. It is shown that we can substitute an arbitrary lagrangian by an homogeneous one using the traditional procedure by adding new variables and, then, this new homogeneous lagrangian has zero energy. The authors show that the hamiltonian can be added as a new constraint and, in consequence, they restrict themselves to the case when the hamiltonian is identically zero. The integrability condition for the resultant Hamilton-Jacobi equations implies that they can only consider first class constraints. On the other hand, in the paper by Rothe and Scholtz ${ }^{37}$ an almost-regular lagrangian $L\left(t, q^{A}, \dot{q}^{A}\right)$ is considered. If the Hessian $\left(\frac{\partial^{2} L}{\partial \dot{q}^{A} \partial \dot{q}^{B}}\right)$, has rank $n-m_{1}$ then, the constraint submanifold $M_{1}$ is locally described by coordinates $\left(q^{A}, p_{a}\right)$, where only $, a=m_{1}+1, \ldots, n$. The remaining momenta $p_{\alpha} ; \alpha=1, \ldots$, $m_{1}$ are functions of $t, q^{A}, p_{a}$, that is, $p_{\alpha}=-f_{\alpha}\left(t, q^{A}, p_{a}\right)$ and represent the primary constraints $\phi_{\alpha}\left(t, q^{A}, p_{A}\right)=p_{\alpha}+f_{\alpha}\left(t, q^{A}, p_{a}\right)$. Then they consider the system of partial differential equations

$$
\begin{aligned}
& \frac{\partial S}{\partial t}+h_{1}\left(t, q^{A}, \frac{\partial S}{\partial q_{a}}\right)=0 \\
& \frac{\partial S}{\partial q^{\alpha}}+f_{\alpha}\left(t, q^{A}, \frac{\partial S}{\partial q_{a}}\right)=0 \quad \alpha=1, \ldots, m_{1}
\end{aligned}
$$

where $h_{1}$ is the hamiltonian defined on the primary constraint manifold by the projection of the lagrangian energy.

(ii) The theory discussed in Ref. 7 is similar to our theory in the case of global dynamics, but they do not take into account secondary constraints. The authors also use the lagrangian homogeneous formalism to obtain the standard Hamilton-Jacobi theory for time dependent systems.

(iii) Leok et al. ${ }^{23}$ use the Dirac structures setting, and secondary constraints are not considered.

\section{H. Lagrangian setting}

The equations of motion are globally expressed by the presymplectic equation

$$
i_{\xi} \omega_{L}=d E_{L}
$$

where a possible solution $\xi$ is not in principle a SODE.

Therefore, in addition to the problem of finding solutions for (4.8), we must study the second order differential problem, that is, we shall obtain a solution of (4.8) satisfying the additional condition $S \xi=\Delta$.

If we apply the constraint algorithm to the presymplectic system $\left(T Q, \omega_{L}, d E_{L}\right)$ we obtain a sequence of submanifolds.

$$
\cdots P_{k} \hookrightarrow \cdots \hookrightarrow P_{2} \hookrightarrow P_{1}:=T Q
$$

Assume that the algorithm stabilizes at some $P_{k+1}=P_{k}=P_{f}$, which is the final constraint submanifold.

If the Lagrangian function is almost-regular, as above, we may consider the presymplectic system $\left(M_{1}, \omega_{1}, d h_{1}\right)$, and apply the constraint algorithm to the equation

$$
i_{X} \omega_{1}=d h_{1},
$$

we obtain a sequence of submanifolds

$$
\cdots M_{k} \hookrightarrow \cdots \hookrightarrow M_{2} \hookrightarrow M_{1} \hookrightarrow T^{*} Q
$$

such that

$$
F L\left(P_{i}\right)=M_{i}, \text { for any } i,
$$


and

$$
F L_{i}:=F L_{\mid P_{i}}: P_{i} \rightarrow M_{i}
$$

are surjective submersions satisfying $F L_{i}^{-1}(y)=F L^{-1}(y)$, for all $y \in M_{i}$.

As a consequence, both algorithms stabilizes at the same step, say $k$, and then

$$
F L\left(P_{f}\right)=M_{f}
$$

and

$$
F L_{f}: P_{f} \rightarrow M_{f}
$$

is a surjective submersion. Moreover, we have the following results.

Proposition 4.18. If $\xi \in \mathfrak{X}\left(P_{f}\right)$ is a $F L_{f}$-projectable solution of (4.8), then its projection $T F L_{f}(\xi)$ is a solution of (4.9).

Conversely, if $X$ is a solution of (4.9), then any $F L_{f}$ projectable vector field on $P_{f}$ which projects on $X$, is a solution of (4.8).

Next, we shall discuss the SODE problem as it was stated by Gotay and Nester ${ }^{14,17}$ (see Ref. 6 for an alternative description).

The results in Refs. 14 and 17 can be summarized in the following result.

\section{Theorem 4.19.}

(i) If $\xi \in \mathfrak{X}\left(P_{f}\right)$ is a $F L_{f}$-projectable vector field on $P_{f}$ then for any $p \in M_{f}$ there exists a unique point in each fiber $F L_{f}^{-1}(p)$, denoted by $\eta_{\xi}(p)$ at which $\xi$ is a SODE. The point $\eta_{\xi}(p)$ is given by

$$
\eta_{\xi}(p):=T \tau_{Q}(\xi(p))
$$

(ii) The map

$$
\begin{aligned}
\beta_{\xi}: M_{f} & \longrightarrow P_{f} \\
p & \rightarrow \beta_{\xi}(p):=\eta_{\xi}(p)
\end{aligned}
$$

is a section of $F L_{f}: P_{f} \rightarrow M_{f}$ and on $\operatorname{Im}\left(\beta_{\xi}\right)$ there exists a unique vector field, denoted by $X_{\xi}$, which simultaneously satisfies the equations

$$
\begin{aligned}
& i_{X_{\xi}} \omega_{L}=d E_{L}, \\
& S X_{\xi}=\Delta .
\end{aligned}
$$

We will now recall the construction of a solution of the dynamical equation which simultaneously satisfies the SODE condition. If $X:=\left(F L_{f}\right)_{*}(\xi)$, then $X$ is a vector field on $M_{f}$ satisfying $i_{X} \omega_{1}=d h_{1}$. The vector field $X_{\xi}$ described in (ii) is given by

$$
X_{\xi}\left(\beta_{\xi}(p)\right)=T \beta_{\xi}(X(p)) \text {, for } p \in M_{f}
$$

A detailed proof can be seen in Refs. 14 and 17, but for the sake of completeness, we recall here the way to choose the points on the fibers as it is stated in the Theorem 4.19 (i).

In the last part of this section we come back to the Hamilton-Jacobi problem, but now in the lagrangian setting. 
The application of the constraint algorithm is summarized in the following diagram:

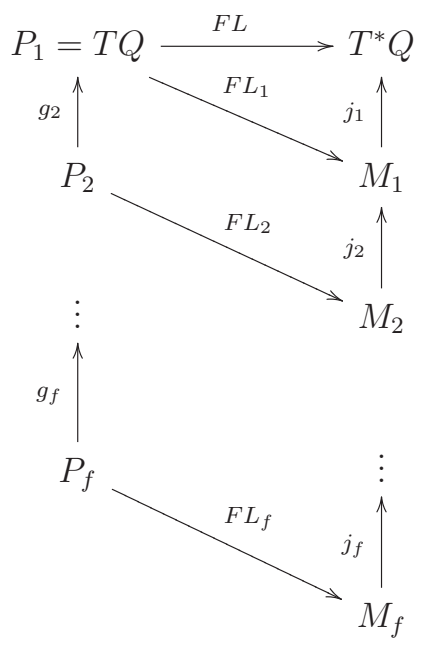

Assume, as before, that $Q_{i}=\pi_{Q}\left(M_{i}\right)$ are submanifolds of $Q$ and $\pi_{i}=\pi_{Q_{\mid M_{i}}: M_{i} \rightarrow Q_{i} \text { are }}$ surjective submersions. Since $\tau_{Q}=\pi_{Q} \circ F L$, then $\tau_{Q}\left(P_{i}\right)=\pi_{Q}\left(M_{i}\right)=Q_{i}$, and $P_{i}$ also projects onto $Q_{i}$. We denote $\tau_{f}=\tau_{Q_{\mid P_{f}}}: P_{f} \rightarrow Q_{f}$.

In consequence, the following diagram is commutative.

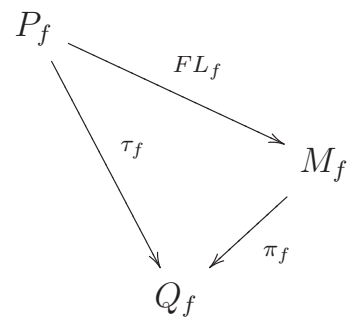

Now, if $X$ is a solution of $i_{X} \omega_{1}=d h_{1}$ on $M_{f}$ and $\gamma$ is a 1 -form which is a solution of the Hamilton-Jacobi problem, that is,

(i) $\quad \gamma(Q) \subset M_{1}$ and $\gamma_{f}\left(Q_{f}\right) \subset M_{f}$,

(ii) $d\left(h_{1} \circ \gamma_{1}\right)_{\mid Q_{f}}=0$,

(iii) $d \gamma=0$,

then we can define $X^{\gamma}=T \pi_{f} \circ X \circ \gamma_{f}$. From Proposition 4.7 we deduce that $X$ and $X^{\gamma}$ are $\gamma_{f}$-related.

On the other hand, we can construct a $F L_{f}$-projectable vector field $\xi$ on $P_{f}$ which projects on $X$. Next we can apply Theorem 4.19 and obtain the section $\beta_{\xi}: M_{f} \rightarrow P_{f}$. Recall that $X_{\xi}\left(\beta_{\xi}(p)\right)$ $=T \beta_{\xi}(X(p))$, for $p \in M_{f}$, is the unique vector field on $\operatorname{Im}\left(\beta_{\xi}\right)$ which satisfies the SODE condition and the equation $i_{X_{\xi}} \omega_{L}=d E_{L}$. The following lemma gives the relation between $\operatorname{Im}\left(\beta_{\xi}\right)$ and $Q_{f}$.

Lemma 4.20. $\operatorname{Im}\left(\beta_{\xi}\right)$ is a submanifold of $T Q_{f}$.

Proof: Since $X_{\xi}$ verifies the SODE condition, then

$$
T \tau_{Q}\left(X_{\xi}\left(\beta_{\xi}(p)\right)\right)=\tau_{T Q}\left(X_{\xi}\left(\beta_{\xi}(p)\right)\right)
$$

for any $p \in M_{f}$.

Since $X_{\xi}$ is tangent to $\operatorname{Im}\left(\beta_{\xi}\right)$, and since $\operatorname{Im}\left(\beta_{\xi}\right)$ is a submanifold of $P_{f}$ and $\tau_{Q}\left(P_{f}\right)=Q_{f}$, then $T \tau_{Q}\left(X_{\xi}\left(\beta_{\xi}(p)\right)\right) \in T Q_{f}$.

On the other hand $\tau_{T Q}\left(X_{\xi}\left(\beta_{\xi}(p)\right)\right)=\beta_{\xi}(p) \in \operatorname{Im}\left(\beta_{\xi}\right)$, and using the SODE condition we deduce that $\beta_{\xi}(p) \in T Q_{f}$. 
Remember that $X_{\xi}$ and $X$ are $\beta_{\xi}$-related and $X$ and $X^{\gamma}$ are $\gamma_{f}$-related, so we deduce that $X_{\xi}$ and $X^{\gamma}$ are $\beta_{\xi} \circ \gamma_{f}$-related too. Moreover, since $X_{\xi}$ satisfies the SODE condition, we can find a better description of $\beta_{\xi} \circ \gamma_{f}$.

Proposition 4.21. We have

$$
\beta_{\xi} \circ \gamma_{f}=X^{\gamma}
$$

Proof: Since $X_{\xi}$ verifies the SODE condition, then given $q \in Q_{f}$ we obtain

$$
T_{q} \tau_{f}\left(X_{\xi}\left(\beta_{\xi} \circ \gamma_{f}(q)\right)\right)=\tau_{T Q}\left(X_{\xi}\left(\beta_{\xi} \circ \gamma_{f}(q)\right)\right) .
$$

Therefore,

$$
T \tau_{Q}\left(X_{\xi}\left(\beta_{\xi} \circ \gamma_{f}(q)\right)\right)=T \tau_{Q} \circ T \beta_{\xi}\left(X\left(\gamma_{f}(q)\right)\right)=T \pi_{Q}(X(\gamma(q)))=X^{\gamma}(q),
$$

where we have used that $\tau_{Q}=\pi \circ F L$ and $F L \circ \beta_{\xi}=i d_{M_{f}}$.

On the other hand,

$$
\tau_{T Q}\left(X_{\xi}\left(\beta_{\xi} \circ \gamma_{f}(q)\right)\right)=\left(\beta_{\xi} \circ \gamma_{f}(q)\right) .
$$

Then, using the SODE condition we get $X^{\gamma}=\beta_{\xi} \circ \gamma_{f}$.

The following corollary is immediate.

Corollary 4.22. The vector fields $X_{\xi}$ and $X^{\gamma}$ are $X^{\gamma}$-related, i.e.,

$$
X_{\xi}\left(\beta_{\xi} \circ \gamma_{f}(q)\right)=T X^{\gamma}\left(X^{\gamma}(q)\right)
$$

or equivalently

$$
X_{\xi}\left(\beta_{\xi} \circ \gamma_{f}(q)\right)=\left(X^{\gamma}\right)^{C}\left(X^{\gamma}(q)\right),
$$

where $\left(X^{\gamma}\right)^{C}$ denotes the complete lift of the vector field $X^{\gamma}$.

\section{Example: Lagrangian setting}

Example 4.23. We will revisit example 4.12 and discuss the Hamilton-Jacobi problem for the Euler-Lagrange equation. The lagrangian function is

$$
L\left(q^{1}, q^{2}, \dot{q}^{1}, \dot{q}^{2}\right)=\frac{1}{2}\left(\dot{q}^{1}\right)^{2}+\dot{q}^{2} q^{1}+\dot{q}^{1} q^{2}
$$

Then $F L$ was given

$$
F L\left(q^{1}, q^{2}, \dot{q}^{1}, \dot{q}^{2}\right)=\left(q^{1}, q^{2}, \dot{q}^{1}+q^{2}, q^{1}\right)
$$

and the primary constraints are

$$
\Phi^{1}\left(q^{A}, p_{A}\right)=p_{2}-q^{1}
$$

So

$$
M_{1}=\left\{\left(q^{1}, q^{2}, p_{1}, p_{2}\right) \in \mathbb{R}^{4} \text { such that } p_{2}=q^{1}\right\} .
$$

and we can use $\left(q^{1}, q^{2}, p_{1}\right)$ as coordinates on $M_{1}$.

It follows that

$$
\begin{aligned}
& E_{L}=\frac{1}{2}\left(\dot{q}^{1}\right)^{2}, \\
& h_{1}=\frac{1}{2}\left(p_{1}-q^{2}\right)^{2}, \\
& \omega_{1}=d q^{1} \wedge d p_{1}+d q^{2} \wedge d q^{1}, \\
& \operatorname{ker}\left(\omega_{1}\right)=\left\langle\frac{\partial}{\partial p_{1}}-\frac{\partial}{\partial q^{2}}\right\rangle .
\end{aligned}
$$


Let

$$
h\left(q^{A}, p_{A}\right)=\frac{1}{2}\left(p_{1}-q^{2}\right)^{2}
$$

be an extension of the hamiltonian $h_{1}$.

It is easy to see that, at the points of $M_{1}:=\operatorname{Im}(F L)$, we have

$$
\left\{\Phi^{1}, h+u \Phi^{1}\right\}=0
$$

and therefore we are in presence of global dynamics

The solution of the equation $\left(i_{X} \omega_{1}=d h_{1}\right)_{\mid M_{1}}$ is given by

$$
X=\left(p_{1}-q^{2}\right) \frac{\partial}{\partial q^{1}}+f \frac{\partial}{\partial q^{2}}+f \frac{\partial}{\partial p_{1}}+\left(p_{1}-q^{2}\right) \frac{\partial}{\partial p^{2}},
$$

where $f \in C^{\infty}\left(M_{1}\right)$

Recall also, that a solution of our Hamilton-Jacobi problem, $\gamma\left(q^{1}, q^{2}\right)=\left(q^{1}, q^{2}, \gamma_{1}\left(q^{1}, q^{2}\right)\right.$, $\left.\gamma_{2}\left(q^{1}, q^{2}\right)\right)$, was given by

$$
\gamma\left(q^{1}, q^{2}\right)=\left(q^{1}, q^{2}, q^{2}, q^{1}\right) .
$$

If we take a solution $X=\left(p_{1}-q^{2}\right) \frac{\partial}{\partial q^{1}}+f \frac{\partial}{\partial q^{2}}+f \frac{\partial}{\partial p_{1}}+\left(p_{1}-q^{2}\right) \frac{\partial}{\partial p_{2}}$ we can compute

$$
X^{\gamma}=0 \frac{\partial}{\partial q^{1}}+f \frac{\partial}{\partial q^{2}}
$$

and also

$$
\left(X^{\gamma}\right)^{C}\left(q^{1}, q^{2}, \dot{q}^{1}, \dot{q}^{2}\right)=(f \circ \gamma) \frac{\partial}{\partial q^{2}}+\left(\left(\frac{\partial(f \circ \gamma)}{\partial q^{1}}\right) \dot{q}^{1}+\left(\frac{\partial(f \circ \gamma)}{\partial q^{2}}\right) \dot{q}^{2}\right) \frac{\partial}{\partial \dot{q}^{2}},
$$

then

$$
\left(X^{\gamma}\right)^{C}\left(X^{\gamma}\right)=\left(X^{\gamma}\right)^{C}\left(q^{1}, q^{2}, 0,(f \circ \gamma)\left(q^{1}, q^{2}\right)\right)=(f \circ \gamma) \frac{\partial}{\partial q^{2}}+\left(\frac{\partial(f \circ \gamma)}{\partial q^{2}}\right) \dot{q}^{2} \frac{\partial}{\partial \dot{q}^{2}} .
$$

This vector field along $X^{\gamma}$ satisfies the SODE condition. We can consider now the equation $i_{\xi} \omega_{L}=d E_{L}$

$$
\begin{aligned}
\omega_{L} & =d\left(\frac{\partial L}{\partial \dot{q}^{1}} d q^{1}+\frac{\partial L}{\partial \dot{q}^{2}} d q^{2}\right)=d\left(\left(\dot{q}^{1}+q^{2}\right) d q^{1}+q^{1} d q^{2}\right) \\
& =d \dot{q}^{1} \wedge d q^{1}+d q^{2} \wedge d q^{1}+d q^{1} \wedge d q^{2}=d \dot{q}^{1} \wedge d q^{1} .
\end{aligned}
$$

So, $i_{\left(X^{\gamma}\right)^{c}\left(X^{\gamma}\right)} \omega_{L}=0$ and $d E_{L} \circ\left(X^{\gamma}\right)=\dot{q}^{1} d \dot{q}^{1} \circ\left(X^{\gamma}\right)=0$ and thus

$$
\left(i_{\left(X^{\gamma}\right)^{c}\left(X^{\gamma}\right)} \omega_{L}=d E_{L}\right)_{\mid \operatorname{Im}\left(X^{\gamma}\right)} .
$$

Therefore $\left(X^{\gamma}\right)^{C}\left(X^{\gamma}\right)$ satisfies Euler-Lagrange equations and the SODE condition in the submanifold $\operatorname{Im}\left(X^{\gamma}\right)$.

\section{ACKNOWLEDGMENTS}

This work has been in part supported by MICINN (Spain) (Grant Nos. MTM2009-13383, MTM2010-21186-C02-01, and MTM2009-08166-E), the ICMAT Severo Ochoa project SEV-20110087 and the European project IRSES-project "Geomech-246981."

${ }^{1}$ R. Abraham and J. E. Marsden, Foundations of Mechanics, 2nd ed. (Benjamin-Cummings, Reading, MA, 1978).

${ }^{2}$ V. I. Arnold, Mathematical Methods of Classical Mechanics, 2nd ed. Graduate Texts in Mathematics, 60 (Springer-Verlag, New York, 1989).

${ }^{3}$ J. Barcelos-Neto and N. R. F. Braga, "Symplectic analysis of a Dirac constrained theory," J. Math. Phys. 35(7), 3497-3503 (1994).

${ }^{4}$ P. G. Bergmann, "Hamilton-Jacobi theory with mixed constraints," Trans. N. Y. Acad. Sci. 33, 108-115 (1971).

${ }^{5}$ K. H. Bhaskara and K. Viswanath, Poisson Algebras and Poisson Manifolds, Pitman Research Notes in Mathematics Series 174 (Longman Scientific \& Technical, Harlow, New York, 1988). 
${ }^{6}$ J. F. Cariñena, "Theory of singular Lagrangians," Fortschr. Phys. 38(9), 641-679 (1990).

${ }^{7}$ J. F. Cariñena, X. Gràcia, G. Marmo, E. Martínez, M. Muñoz-Lecanda, and N. Román-Roy, "Geometric Hamilton-Jacobi theory," Int. J. Geom. Methods Mod. Phys. 3(7), 1417-1458 (2006).

${ }^{8}$ J. F. Cariñena, X. Gràcia, G. Marmo, E. Martínez, M. Muñoz-Lecanda, and N. Román-Roy, "Geometric Hamilton-Jacobi theory for nonholonomic dynamical systems," Int. J. Geom. Methods Mod. Phys. 7(3), 431-454 (2010).

${ }^{9}$ J. F. Cariñena, X. Gràcia, G. Marmo, E. Martínez, M. Muñoz-Lecanda, and N. Román-Roy, "Hamilton-Jacobi theory and the evolution operator," Mathematical Physics and Field Theory, Julio Abad, in Memoriam, 1st ed. edited by M. Asorey, J. V. Garcia-Esteve, M. F. Rañada, and J. Sesma (Prensas Univ. Zaragoza, Zaragoza, 2009), pp. 177-186.

${ }^{10}$ P. A. M. Dirac, Lectures on Quantum Mechanics, Belfer Graduate School of Science Monographs Series 2 (Academic Press, Inc., New York, 1967), second printing of the 1964 original.

${ }^{11}$ R. J. Eden, "The Hamiltonian dynamics of non-holonomic systems," Proc. R. Soc. London, Ser. A 205, 564-583 (1951).

${ }^{12}$ R. J. Eden, "The quantum mechanics of non-holonomic systems," Proc. R. Soc. London, Ser. A 205, 583-595 (1951).

${ }^{13}$ C. Godbillon, Géométrie différentielle et mécanique analytique (Hermann, Paris, 1969), p. 183.

${ }^{14}$ M. J. Gotay, "Presymplectic manifolds, geometric constraint theory and the Dirac-Bergmann theory of constraints," Ph.D. dissertation (University of Maryland, 1979).

${ }^{15}$ M. J. Gotay, "On the validity of Dirac's conjecture regarding first-class secondary constraints," J. Phys. A 16(5), L141-L145 (1983).

${ }^{16}$ M. J. Gotay and J. M. Nester, "Presymplectic Lagrangian systems. I. The constraint algorithm and the equivalence theorem," Ann. Inst. Henri Poincare, Sect. A 30(2), 129-142 (1979).

${ }^{17}$ M. J. Gotay and J. M. Nester, "Presymplectic Lagrangian systems. II. The second-order equation problem," Ann. Inst. Henri Poincare, Sect. A 32, 1-13 (1980).

${ }^{18}$ M. J. Gotay and J. M. Nester, "Generalized constraint algorithm and special presymplectic manifolds," Geometric Methods in Mathematical Physics (Proc. NSF-CBMS Conf., Univ. Lowell, Lowell, Mass., 1979), Lecture Notes in Mathematics, 775 (Springer, Berlin, 1980), pp. 78-104.

${ }^{19}$ M. J. Gotay and J. M. Nester, "Apartheid in the Dirac theory of constraints," J. Phys. A 17(15), 3063-3066 (1984).

${ }^{20}$ M. J. Gotay, J. M. Nester, and G. Hinds, "Presymplectic manifolds and the Dirac-Bergmann theory of constraints," J. Math. Phys. 19(11), 2388-2399 (1978).

${ }^{21}$ A. Ibort, M. de León, J. C. Marrero, and D. Martín de Diego, "Dirac brackets in constrained dynamics," Fortschr. Phys. 47(5), 459-492 (1999).

${ }^{22}$ O. Krupková, “A geometric setting for higher-order Dirac-Bergmann theory of constraints,” J. Math. Phys. 35(12), 65576576 (1994).

${ }^{23}$ M. Leok, T. Ohsawa, and D. Sosa, "Hamilton-Jacobi theory for degenerate Lagrangian systems with holonomic and nonholonomic constraints," J. Math. Phys. 53, 072905 (2012).

${ }^{24}$ M. de León, D. Iglesias-Ponte, and D. Martín de Diego, "Towards a Hamilton-Jacobi theory for nonholonomic mechanical systems," J. Phys. A: Math. Theor. 41, 015205 (2008).

${ }^{25}$ M. de León, J. C. Marrero, and D. Martín de Diego, "A geometric Hamilton-Jacobi theory for classical field theories," Variations, Geometry and Physics (Nova Sci. Publ., New York, 2009), pp. 129-140.

${ }^{26}$ M. de León, J. C. Marrero, and D. Martín de Diego, "Linear almost Poisson structures and Hamilton-Jacobi equation. Applications to nonholonomic mechanics," J. Geom. Mech. 2(2), 159-198 (2010).

${ }^{27}$ M. de León, D. Martín de Diego, and P. Pitanga, "A new look at degenerate Lagrangian dynamics from the viewpoint of almost-product structures," J. Phys. A 28(17), 4951-4971 (1995).

${ }^{28}$ M. de León and D. Martín de Diego, "A constraint algorithm for singular Lagrangians subjected to nonholonomic constraints," J. Math. Phys. 38(6), 3055-3062 (1997).

${ }^{29}$ M. de León, D. Martín de Diego, and M. Vaquero, "A geometric Hamilton-Jacobi theory for multisymplectic field theories" (to be published).

${ }^{30}$ M. de León and P. R. Rodrigues, Methods of Differential Geometry in Analytical Mechanics, North-Holland Mathematics Studies, 158 (North-Holland Publishing Co., Amsterdam, 1989).

${ }^{31}$ G. Longhi, D. Dominici, J. Gomis, and J. M. Pons, "The Hamilton-Jacobi formalism for systems with constraints," Relativistic Action at a Distance: Classical and Quantum Aspects (Barcelona, 1981), Lecture Notes in Physics (Springer, Berlin, 1982), Vol. 162, pp. 165-189.

${ }^{32}$ G. Longhi, D. Dominici, J. Gomis, and J. M. Pons, "Hamilton-Jacobi theory for constrained systems," J. Math. Phys. 25(8), 2439-2452 (1984).

${ }^{33}$ G. Marmo, G. Morandi, and N. Mukunda, "The Hamilton-Jacobi theory and the analogy between classical and quantum mechanics," J. Geom. Mech. 1(3), 317-355 (2009).

${ }^{34}$ G. Marmo, N. Mukunda, and J. Samuel, "Dynamics and symmetry for constrained systems: a geometrical analysis," Riv. Nuovo Cimento 6(2), 1-62 (1983).

${ }^{35}$ J. C. Marrero and D. Sosa, "The Hamilton-Jacobi equation on Lie affgebroids," Int. J. Geom. Methods Mod. Phys. 3, 605-622 (2006).

${ }^{36}$ T. Ohsawa and A. Bloch, "Nonholonomic Hamilton-Jacobi equation and integrability,” J. Geom. Mech. 1(4), 461-481 (2009).

${ }^{37}$ K. D. Rothe and F. G. Scholtz, "On the Hamilton-Jacobi equation for second-class constrained systems," Ann. Phys. 308, 639-651 (2003).

${ }^{38}$ H. Rund, The Hamilton-Jacobi Theory in the Calculus of Variations, D. Van Nostrand Co., Ltd., London-Toronto, Ont.-New York, 1966 
${ }^{39}$ R. Skinner and R. Rusk, "Generalized Hamiltonian dynamics. I. Formulation on $T^{*} Q \oplus T Q$," J. Math. Phys. 24(11), 2589-2594 (1983).

${ }^{40}$ E. C. G. Sudarshan and N. Mukunda, Classical Dynamics: A Modern Perspective (Wiley-Interscience, New York/London/Sydney, 1974).

${ }^{41}$ K. Sundermeyer, Constrained dynamics. With applications to Yang-Mills Theory, General Relativity, Classical Spin, Dual String Model, Lecture Notes in Physics, 169 (Springer-Verlag, Berlin/New York, 1982). 


\title{
A HAMILTON-JACOBI THEORY FOR SINGULAR LAGRANGIAN SYSTEMS IN THE SKINNER AND RUSK SETTING
}

\author{
MANUEL DE LEÓN*, DAVID MARTÍN DE DIEGO ${ }^{\dagger}$ \\ and MIGUEL VAQUERO $\ddagger$

Received 3 May 2012

Accepted 26 June 2012

Published 9 October 2012

\begin{abstract}
We develop a Hamilton-Jacobi theory for singular Lagrangian systems in the SkinnerRusk formalism. Comparisons with the Hamilton-Jacobi problem in the Lagrangian and Hamiltonian settings are discussed.

Keywords: Hamilton-Jacobi theory; presymplectic constraint algorithm.
\end{abstract}

\section{Contents}

1 Introduction 2

2 Notation and Background 3

3 The Skinner and Rusk Formalism $\quad 7$

4 A Hamilton-Jacobi Theory in the Skinner-Rusk Setting 9

4.1 The regular case 9

4.2 The singular case 10

5 Comparison with the Hamiltonian and Lagrangian Settings 11

$\begin{array}{ll}5.1 \text { The Hamiltonian setting } & 12\end{array}$

5.1.1 The regular case 12

5.1.2 The singular case $\quad 12$

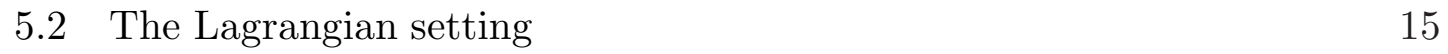

5.2.1 The regular case 16

5.2.2 The singular case 16

6 Final Considerations 20

Appendix A. The Gotay-Nester-Hinds Algorithm of Constraints 22 
Acknowledgments 23

References $\quad 23$

\section{Introduction}

The standard formulation of the Hamilton-Jacobi problem is to find a function $S\left(t, q^{A}\right)$ (called the principal function) such that

$$
\frac{\partial S}{\partial t}+h\left(q^{A}, \frac{\partial S}{\partial q^{A}}\right)=0
$$

where $h=h\left(q^{A}, p_{A}\right)$ is the Hamiltonian function of the system. If we put $S\left(t, q^{A}\right)=$ $W\left(q^{A}\right)-t E$, where $E$ is a constant, then $W$ satisfies

$$
h\left(q^{A}, \frac{\partial W}{\partial q^{A}}\right)=E
$$

$W$ is called the characteristic function.

Equations (1.1) and (1.2) are indistinctly referred as the Hamilton-Jacobi equation (see $[1,2,23])$.

This theory works for classical mechanical systems, where the Lagrangian function is usually the kinetic energy corresponding to a Riemannian metric on the configuration manifold minus a potential energy. This is the case of the so-called regular Lagrangian systems, that have a well-defined Hamiltonian counterpart. The theory has been recently reformulated in a geometrical setting (see [3-5]) that has permitted its extension to nonholonomic mechanical systems [13, 15], and even classical field theories $[14,18,26]$. The procedure is based on the comparison of the Hamiltonian vector field $X_{h}$ on the cotangent bundle $T^{*} Q$ and its projection onto $Q$ via a closed 1-form $\gamma$ on $Q$; the result says that both vector fields are $\gamma$-related if and only if the Hamilton-Jacobi equations $d(h \circ \gamma)=0$ holds.

On the other hand, a Hamilton-Jacobi theory for singular Lagrangian systems is far to be accomplished. There were several attempts (see [20-22]), based on the homogenization of the given Lagrangian, which leads to a new Lagrangian system with null energy such that it is possible to discuss the Hamilton-Jacobi equation for the constraints themselves. The main problem is that, due to the integrability condition for the resultant partial differential equation, one can only consider first class constraints. Therefore, the treatment of the cases when second class constraints appear should be developed by ad hoc arguments (as in [22], for instance). Thus, in [20] and [21] the authors only discuss the case of primary constraints.

A more modern discussion on this subject can be found in $[3,12]$, but these authors only consider the case of primary constraints. More recently, in [17] it is proposed a Hamilton-Jacobi theory for arbitrary singular systems that works even if the system exhibit secondary constraints. The strategy is to apply the geometric procedure described above in combination with the constraint algorithm developed 
by Gotay and Nester [7-10] and that geometrizes the well-known Dirac theory of constraints [6].

In the present paper we take a different approach, and consider the Skinner and Rusk setting to treat with singular Lagrangians [24, 25]. Skinner and Rusk have considered a geometrized framework where the velocities and the momenta are independent coordinates. To do this, they considered the dynamics on the Whitney sum of $T Q$ (the space of velocities) and $T^{*} Q$ (the phase space).

Given a Lagrangian function $L: T Q \rightarrow \mathbb{R}$ (singular or regular, no matter) one considers the bundle $T Q \oplus T^{*} Q$ with canonical projections $\operatorname{pr}_{1}: T Q \oplus T^{*} Q \rightarrow T Q$ and $\mathrm{pr}_{2}: T Q \oplus T^{*} Q \rightarrow T^{*} Q$ onto the first and second factors. We then define a function $D: T Q \oplus T^{*} Q \rightarrow \mathbb{R}$ by $D\left(X_{p}, \alpha_{p}\right)=\alpha_{p}\left(X_{p}\right)-L\left(X_{p}\right)$. In bundle coordinates $\left(q^{A}, v^{A}, p_{A}\right), D$ is given by $D\left(q^{A}, v^{A}, p_{A}\right)=v^{A} p_{A}-L\left(q^{A}, v^{A}\right)$, and it is sometimes referred as the Pontryagin Hamiltonian or generalized energy (see [27]). We can also define a 2 -form $\Omega$ on $T Q \oplus T^{*} Q$ by $\Omega=\operatorname{pr}_{2}^{*}\left(\Omega_{Q}\right)$, where $\Omega_{Q}$ denotes the canonical symplectic 2 -form of $T^{*} Q$.

Then, one discuss the presymplectic system $\left(T Q \oplus T^{*} Q, \Omega, d D\right)$ and obtain the corresponding sequence of constraint submanifolds, which, of course, have a close relation with those obtained by Gotay and Nester on the Lagrangian and Hamiltonian sides. It should be noticed that this algorithm includes the SODE condition just from the very beginning.

We apply the Hamilton-Jacobi geometric procedure to this presymplectic system and develop the corresponding Hamilton-Jacobi theory. The relation with the Hamilton-Jacobi problems on the Lagrangian and Hamiltonian sides are extensively discussed.

\section{Notation and Background}

In this work all manifolds are assumed to be finite-dimensional and $C^{\infty}$. Given a function $f$, the differential at a point $p$ will be indistinctly denoted by $d_{p} f$ or $d f(p)$.

We refer to [19] for a detailed description of Lagrangian and Hamiltonian mechanical systems.

Let $Q$ be a differentiable manifold and denote by $T Q$ and $T^{*} Q$ the tangent and cotangent bundles, and by $\tau_{Q}: T Q \rightarrow Q$ and $\pi_{Q}: T^{*} Q \rightarrow Q$ the respective canonical projections onto $Q$.

We introduce two canonical structures on the tangent bundle of a manifold: the vertical endomorphism $S$, and the Liouville vector field $\Delta$. In bundle coordinates, $\left(q^{A}, v^{A}\right)$, they are respectively given by

$$
\begin{aligned}
& S=d q^{A} \otimes \frac{\partial}{\partial v^{A}}, \\
& \Delta=v^{A} \frac{\partial}{\partial v^{A}} .
\end{aligned}
$$


Let now $L: T Q \rightarrow \mathbb{R}$ be a Lagrangian on $T Q$; we can define the Poincaré-Cartan 2 -form and the energy function of $L$ by

$$
\begin{aligned}
& \Omega_{L}=-d \theta_{L}, \quad \text { where } \theta_{L}=S^{*}(d L), \\
& E_{L}=\Delta(L)-L,
\end{aligned}
$$

which in local coordinates read as

$$
\begin{aligned}
\theta_{L} & =\frac{\partial L}{\partial v^{A}} d q^{A} \\
\Omega_{L} & =d q^{A} \wedge d\left(\frac{\partial L}{\partial v^{A}}\right), \\
E_{L} & =v^{A} \frac{\partial L}{\partial v^{A}}-L(q, v) .
\end{aligned}
$$

We look for vector fields $\xi$ which simultaneously satisfy the equations

$$
\begin{aligned}
i_{\xi} \Omega_{L} & =d E_{L}, \\
S \xi & =\Delta .
\end{aligned}
$$

If the Lagrangian $L$ is regular, that is, $\operatorname{det}\left(\frac{\partial^{2} L}{\partial v^{A} \partial v^{B}}\right) \neq 0$, then the form $\Omega_{L}$ is symplectic $\left(\Omega_{L}\right.$ has maximal rank) and there exists a unique vector field $\xi$ on $T Q$ which satisfies Eq. (2.1). This vector field automatically satisfies the SODE condition (2.2).

If the Lagrangian is not regular, then $\Omega_{L}$ is no longer symplectic and Eq. (2.1) has no solution in general and even if there is a solution it is not necessary a SODE. Therefore for a singular Lagrangian $L, \Omega_{L}$ is a presymplectic form (that is, the rank is not maximal, although, for simplicity, it is assumed that it is constant).

We define the Legendre transformation associated to $L$ as the mapping

$$
\begin{aligned}
F L: \quad T Q & \rightarrow T^{*} Q \\
\left(q^{A}, v^{A}\right) & \rightarrow F L\left(q^{A}, v^{A}\right)=\left(q^{A}, \frac{\partial L}{\partial v^{A}}\left(q^{A}, v^{A}\right)\right) .
\end{aligned}
$$

From a direct inspection in local coordinates we know that the Legendre transformation is a local diffeomorphism if and only if $L$ is regular.

We can apply the Gotay-Nester-Hinds algorithm of constraints, see [7-9], to the presymplectic system $\left(T Q, \Omega_{L}, d E_{L}\right)$ and hence we obtain a sequence of constraint submanifolds

$$
\cdots P_{k} \hookrightarrow \cdots \hookrightarrow P_{2} \hookrightarrow P_{1}=T Q
$$

Assume that the algorithm stabilizes at some step $k$, say $P_{k+1}=P_{k}$, which is called the final constraint submanifold, denoted by $P_{f}=P_{k}$. 
In this paper we will only consider almost regular Lagrangians $L: T Q \rightarrow \mathbb{R}$, that is:

(i) $M_{1}=\operatorname{Im}(\mathbb{F} L)$ is a submanifold of $T^{*} Q$, and

(ii) $F L: T Q \rightarrow \operatorname{Im}(\mathbb{F} L)$ is a surjective submersion with connected fibers.

Under these assumptions, the energy $E_{L}$ is projected onto a function $h_{1}: M_{1} \rightarrow$ $\mathbb{R}$ such that $h_{1} \circ F L=E_{L}$

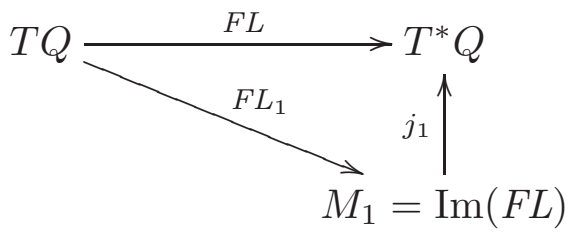

Here $F L_{1}$ is the restriction of $F L$ to its image, and $j_{1}: M_{1} \rightarrow T^{*} Q$ is the canonical inclusion.

Next, we shall study the presymplectic system given by $\left(M_{1}, \Omega_{1}=j_{1}^{*} \Omega_{Q}, d h_{1}\right)$, where $\Omega_{Q}$ is the canonical symplectic form on $T^{*} Q$. Therefore, we consider the equation

$$
i_{Y} \Omega_{1}=d h_{1} .
$$

As above we can apply the presymplectic algorithm and obtain a sequence of constraint submanifolds

$$
\cdots M_{k} \hookrightarrow \cdots \hookrightarrow M_{2} \hookrightarrow M_{1} \hookrightarrow T^{*} Q
$$

It is obvious that

$$
F L\left(P_{i}\right)=M_{i}, \quad \text { for any } i
$$

and, furthermore, the induced mappings

$$
F L_{i}=F L_{\mid P_{i}}: P_{i} \rightarrow M_{i}
$$

are surjective submersions, for all $i$.

Hence, both algorithms stabilizes at the same step, say $k$, and then

$$
F L\left(P_{f}\right)=M_{f}
$$

and

$$
F L_{f}: P_{f} \rightarrow M_{f}
$$

is a surjective submersion (with the obvious notations). 
The following diagram summarizes the above discussion.

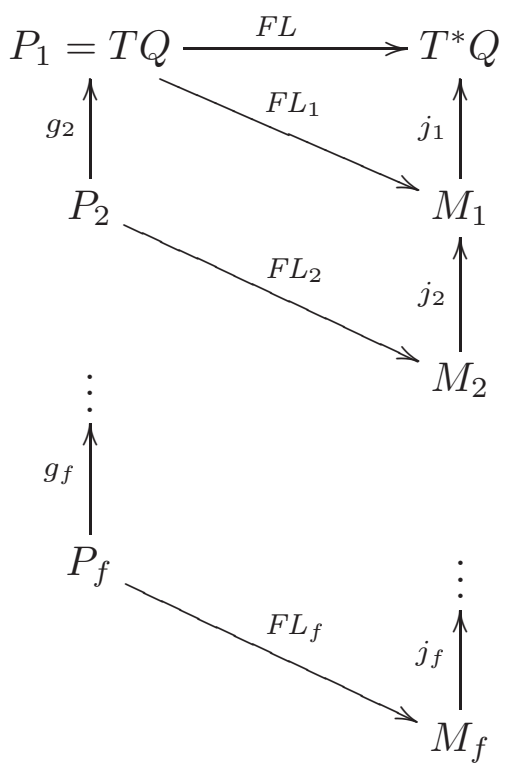

where $g_{i}$ and $j_{i}$ denote the natural inclusions.

The relation between Eqs. (2.1) and (2.3) is given by the following theorem.

Proposition 2.1. If $\xi \in T_{p} T Q$ satisfies $(2.1)$, then $T F L(\xi) \in T_{F L(p)} M_{1}$ satisfies (2.3). Therefore, if $\xi$ is a $F L_{f}$-projectable solution of (2.1), then its projection $F L_{f_{*}}(\xi)$ is a solution of (2.3).

Conversely, if $Y$ is a solution of (2.1), then any $F L_{f}$ projectable vector field on $P_{f}$ which projects on $Y$, is a solution of (2.3).

Next, we shall discuss the SODE problem as it was stated by Gotay and Nester $[7,8]$.

The results can be summarized in the following result.

Theorem 2.2. (i) If $\xi$ is a $F L_{f}$-projectable vector field on $P_{f}$ then for any $p \in M_{f}$ there exists a unique point in each fiber $F L_{f}^{-1}(p)$, denoted by $\eta_{\xi}(p)$ at which $\xi$ is a SODE. The point $\eta_{\xi}(p)$ is given by

$$
\eta_{\xi}(p)=T \tau_{Q}(\xi(p))
$$

(ii) The map

$$
\begin{aligned}
\beta_{\xi}: M_{f} & \rightarrow P_{f} \\
p & \rightarrow \beta_{\xi}(p)=\eta_{\xi}(p)
\end{aligned}
$$

is a section of $F L_{f}: P_{f} \rightarrow M_{f}$ and on $\operatorname{Im}\left(\beta_{\xi}\right)$ there exists a unique vector field, denoted by $Y_{\xi}$, which simultaneously satisfies the equations

$$
i_{Y_{\xi}} \Omega_{L}=d E_{L}, \quad S Y_{\xi}=\Delta
$$


We will now recall the construction of the solution of the dynamical equation which simultaneously satisfies the SODE condition. If $Y=\left(F L_{f}\right)_{*}(\xi)$, then $Y$ is a vector field on $M_{f}$ satisfying $i_{Y} \Omega_{1}=d h_{1}$. The vector field $Y_{\xi}$ described in (ii) is given by

$$
Y_{\xi}\left(\beta_{\xi}(p)\right)=T \beta_{\xi}(Y(p)), \quad \text { for all } p \in M_{f} .
$$

A detailed discussion can be found in $[19,7-9,11]$.

\section{The Skinner and Rusk Formalism}

Skinner and Rusk [24, 25], have considered a geometrized framework where the velocities and the momenta are independent coordinates. Indeed, they considered the dynamics on the Whitney sum of $T Q$ (the space of velocities) and $T^{*} Q$ (the phase space).

In this section we will briefly recall the Skinner and Rusk formalism.

Let $Q$ be a differentiable manifold and $L: T Q \rightarrow \mathbb{R}$ a Lagrangian. We can consider the bundle $T Q \oplus T^{*} Q$ given by the Whitney sum of $\tau_{Q}: T Q \rightarrow Q$ and $\pi_{Q}: T^{*} Q \rightarrow Q$. We will denote by $\operatorname{pr}_{1}: T Q \oplus T^{*} Q \rightarrow T Q$ and $\operatorname{pr}_{2}: T Q \oplus T^{*} Q \rightarrow$

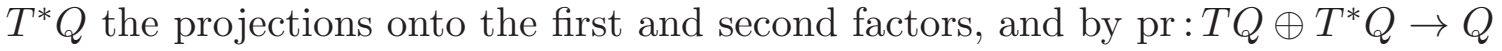
the projection onto $Q$. We then have the following commutative diagram

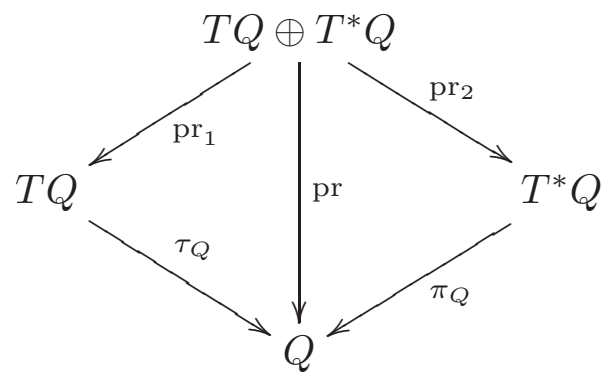

We can define a function

$$
\begin{aligned}
D: T Q \oplus T^{*} Q & \rightarrow \mathbb{R} \\
\left(X_{p}, \alpha_{p}\right) & \rightarrow D\left(X_{p}, \alpha_{p}\right)=\alpha_{p}\left(X_{p}\right)-L\left(X_{p}\right) .
\end{aligned}
$$

In bundle coordinates $\left(q^{A}, v^{A}, p_{A}\right), D$ is given by $D\left(q^{A}, v^{A}, p_{A}\right)=v^{A} p_{A}-L\left(q^{A}, v^{A}\right)$. The function $D$ is sometimes referred as the Pontryagin Hamiltonian or generalized energy (see [27]).

We can define a 2-form $\Omega$ on $T Q \oplus T^{*} Q$ by $\Omega=\operatorname{pr}_{2}^{*}\left(\Omega_{Q}\right)$, where $\Omega_{Q}$ denotes the canonical symplectic 2 -form of $T^{*} Q$.

Next, we can consider the presymplectic system given by $\left(W_{0}=T Q \oplus\right.$ $\left.T^{*} Q, \Omega, d D\right)$ and study the equation

$$
i_{X} \Omega=d D
$$


applying the Gotay-Nester-Hinds algorithm of constraints. Hence, we obtain

$$
W_{1}=\left\{x \in W_{0} \text { such that there exists } X \in T_{x} W_{0} \text { satisfying } i_{X} \Omega=d D\right\} .
$$

In canonical coordinates $\left(q^{A}, v^{A}, p_{A}\right)$, we have

$$
\begin{aligned}
\Omega & =d q^{A} \wedge d p_{A}, \\
d D & =-\frac{\partial L}{\partial q^{A}} d q^{A}+\left(p_{A}-\frac{\partial L}{\partial v^{A}}\right) d v^{A}+v^{A} d p_{A} .
\end{aligned}
$$

So, given a tangent vector $X=a^{A} \frac{\partial}{\partial q^{A}}+b^{A} \frac{\partial}{\partial v^{A}}+c_{A} \frac{\partial}{\partial p_{A}} \in T_{\left(q^{A}, v^{A}, p_{A}\right)} W_{0}$ we deduce that

$$
i_{X} \Omega=-c_{A} d q^{A}+a^{A} d p_{A}
$$

and (3.2) is equivalent to the following conditions

$$
\begin{aligned}
a^{A} & =v^{A}, \\
c_{A} & =\frac{\partial L}{\partial q^{A}}, \\
p_{A}-\frac{\partial L}{\partial v^{A}} & =0, \quad 1 \leq A \leq n .
\end{aligned}
$$

Next, we should restrict the dynamics to $W_{1}=\left\{\left(q^{A}, v^{A}, p_{A}\right) \in W_{0}\right.$ such that $\left.p_{A}=\frac{\partial L}{\partial v^{A}}\right\}$, that is, $W_{1}=\operatorname{graph}(F L)$, where $F L: T Q \rightarrow T^{*} Q$ has been defined in Sec. 2.

Accordingly with the Gotay-Nester-Hinds algorithm, a solution $X$ must be tangent to $W_{1}$. Assume that such $X$ has the local expression

$$
X=\bar{a}^{A} \frac{\partial}{\partial q^{A}}+\bar{b}^{A} \frac{\partial}{\partial v^{A}}+\left(\frac{\partial^{2} L}{\partial v^{A} \partial q^{B}} \bar{a}^{B}+\frac{\partial^{2} L}{\partial v^{A} \partial v^{B}} \bar{b}^{B}\right) \frac{\partial}{\partial p_{A}} .
$$

Then, taking into account (3.3) and (3.4), we deduce

$$
\begin{gathered}
\bar{a}^{A}=v^{A}, \\
\frac{\partial^{2} L}{\partial v^{A} \partial q^{B}} v^{B}+\frac{\partial^{2} L}{\partial v^{A} \partial v^{B}} \bar{b}^{B}=\frac{\partial L}{\partial q^{A}} .
\end{gathered}
$$

If there exists such a vector field $X$ tangent to $W_{1}$, satisfying the above conditions, we have done, and the final constraint manifold $W_{f}$ is just $W_{1}$. For instance, if the Lagrangian is regular, $\operatorname{det}\left(\frac{\partial^{2} L}{\partial v^{B} \partial v^{A}}\right) \neq 0$, we can compute $\bar{b}^{A}$ explicitly. If we denote by $C_{A B}$ the matrix $C_{A B}=\left(\frac{\partial^{2} L}{\partial v^{B} \partial v^{A}}\right)$ and $C^{A B}$ its inverse, then

$$
\bar{b}^{A}=-C^{A B}\left(v^{A} \frac{\partial^{2} L}{\partial v^{B} \partial q^{A}}-\frac{\partial L}{\partial q^{A}}\right) .
$$


Otherwise, we need to continue the process, and then we obtain a sequence of submanifolds

$$
\cdots \hookrightarrow W_{k} \hookrightarrow \cdots \hookrightarrow W_{2} \hookrightarrow W_{1} \hookrightarrow W_{0}=T Q \oplus T^{*} Q .
$$

If the algorithm stabilizes, that is, there exists $k$ such that $W_{k}=W_{k+1}$, then $W_{k}$ is called the final constraint submanifold and denoted by $W_{f}$.

\section{A Hamilton-Jacobi Theory in the Skinner-Rusk Setting}

In this section we will develop a Hamilton-Jacobi theory in the Skinner-Rusk formalism. We will use the same notation introduced in the previous sections and discuss separately the regular and the singular cases.

\subsection{The regular case}

Assume that we begin with a regular Lagrangian $L: T Q \rightarrow \mathbb{R}$. Then, $W_{f}=W_{1}$.

A section of $T Q \oplus T^{*} Q$ is given by $\sigma=(Z, \gamma)$ where $Z$ and $\gamma$ are a vector field and a 1 -form on $Q$, respectively. Assume that $\sigma$ satisfies the following conditions:

(i) $\operatorname{Im}(\sigma) \subset W_{1}=\operatorname{graph}(F L)$, and

(ii) $d\left(\operatorname{pr}_{2} \circ \sigma\right)=d \gamma=0$.

Then, by the regularity of $L$, we know that there exists a unique vector field on $W_{1}$, say $X$, satisfying

$$
i_{X} \Omega=d D
$$

and then we can define a vector field on $Q$ by

$$
X^{\sigma}(p)=T \operatorname{pr}(X(\sigma(p))), \quad \text { for all } p \in Q .
$$

Now we have the following proposition.

Proposition 4.1. Under the previous conditions, $d(D \circ \sigma)=0$ if and only if the vector fields $X$ and $X^{\sigma}$ are $\sigma$-related.

Proof. " $\Rightarrow$ " Assume that $d(D \circ \sigma)=0$ holds, then we will prove first that $\left(i_{\left(X-T \sigma\left(X^{\sigma}\right)\right)} \Omega=0\right)_{\mid \operatorname{Im}(\sigma)}$.

It is clear that if $x \in \operatorname{Im}(\sigma)$ then $T_{x}\left(T Q \oplus T^{*} Q\right)=T_{x} \operatorname{Im}(\sigma)+V$, where $V$ denotes the vertical bundle of the projection $\operatorname{pr}: T Q \oplus T^{*} Q \rightarrow Q$. We will show that $i_{\left(X-T \sigma\left(X^{\sigma}\right)\right)} \Omega$ annihilates $T_{x} \operatorname{Im}(\sigma)$ and $V$. Indeed, by the definition of $\Omega$, it is obvious that $\Omega$ vanishes acting on two elements of $V$. Since $X-T \sigma\left(X^{\sigma}\right)$ is vertical, we have

$$
\left(i_{\left(X-T \sigma\left(X^{\sigma}\right)\right)} \Omega\right)(V)=0 .
$$

Given $p \in Q$, since $X$ is a solution on $W_{1}$, we get

$$
\left(i_{X(p)} \Omega\right) \circ T \sigma(p)=d D(\sigma(p)) \circ T \sigma(p)=d(D \circ \sigma)(p)=0 .
$$


On the other hand, $\left(i_{T \sigma\left(X^{\sigma}(p)\right)} \Omega\right) \circ T \sigma(p)=0$ since for any $Y \in T_{p} Q$ we have

$$
\begin{aligned}
\left(i_{T \sigma}\left(X^{\sigma}(p)\right)\right. & \Omega)(T \sigma(p)(Y)) \\
& =\Omega\left(T \sigma\left(X^{\sigma}(p)\right), T \sigma(Y)\right) \\
& =\operatorname{pr}_{2}^{*}\left(\Omega_{Q}\right)\left(T \sigma\left(X^{\sigma}(p)\right), T \sigma(Y)\right) \\
& =\left(\Omega_{Q}\right)\left(T \operatorname{pr}_{2} \circ T \sigma\left(X^{\sigma}(p)\right), T \operatorname{pr}_{2} \circ T \sigma(Y)\right)=\left(\Omega_{Q}\right)\left(T \gamma\left(X^{\sigma}(p)\right), T \gamma(Y)\right) \\
& =-d \gamma\left(\left(X^{\sigma}(p)\right),(Y)\right) \\
& =0
\end{aligned}
$$

and so, we conclude that

$$
\left(i_{\left(X-T \sigma\left(X^{\sigma}\right)\right)} \Omega\right)(T \operatorname{Im}(\sigma))_{\mid \operatorname{Im}(\sigma)}=0
$$

which implies

$$
\begin{aligned}
& \left(i_{(X-}-T \sigma\left(X^{\sigma}\right)\right) \\
& \quad \Omega)(V+T \operatorname{Im}(\sigma))_{\mid \operatorname{Im}(\sigma)} \\
& \quad=\left(i_{\left(X-T \sigma\left(X^{\sigma}\right)\right)} \Omega\right)\left(T\left(T Q \oplus T^{*} Q\right)\right)_{\mid \operatorname{Im}(\sigma)}=0 .
\end{aligned}
$$

Therefore $X-T \sigma\left(X^{\sigma}\right) \in \operatorname{ker}(\Omega)$. This means that $i_{\left(X-T \sigma\left(X^{\sigma}\right)\right)} \Omega=0$, and hence $\iota^{*}\left(i_{\left(X-T \sigma\left(X^{\sigma}\right)\right)} \Omega\right)=i_{\left(X-T \sigma\left(X^{\sigma}\right)\right)}\left(i^{*} \Omega\right)=0$, where $\iota: W_{1} \rightarrow W_{0}$ is the canonical inclusion.

It is not hard to see, that if $L$ is regular then $i^{*} \Omega$ is symplectic and so $(X=$ $\left.T \sigma\left(X^{\sigma}\right)\right)_{\mid \operatorname{Im}(\sigma)}$.

"६" Since $\left(\left(i_{\left(X-T \sigma\left(X^{\sigma}\right)\right)} \Omega\right) \circ T \sigma=d(D \circ \sigma)\right)_{\mid \operatorname{Im}(\sigma)}$, if $X=T \sigma\left(X^{\sigma}\right)$, then $d(D \circ$ $\sigma)=0$.

\subsection{The singular case}

Assume now that $L: T Q \rightarrow \mathbb{R}$ is an almost regular singular Lagrangian.

Suppose that the algorithm of Gotay-Nester-Hinds applied to $\left(W_{0}=T Q \oplus\right.$ $\left.T^{*} Q, \Omega, d D\right)$ stabilizes at a final constraint submanifold $W_{f}$. By construction, there exists at least one vector field $X$ on $W_{f}$ such that

$$
\left(i_{X} \Omega=d D\right)_{\mid W_{f}}
$$

We need some regularity conditions, thus we will also assume that $Q_{i}=\operatorname{pr}\left(W_{i}\right)$ are submanifolds and that $\operatorname{pr}_{i}=\operatorname{pr}_{\mid W_{i}}: W_{i} \rightarrow Q_{i}$ are submersions.

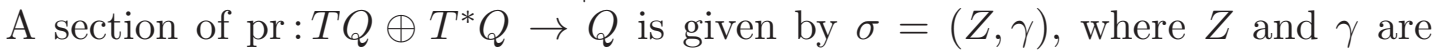
respectively a vector field and a 1 -form on $Q$. We will denote by $\sigma_{f}$ the restriction of $\sigma$ to $Q_{f}=\operatorname{pr}\left(W_{f}\right)$ of $\sigma$. Suppose that $\sigma$ verifies the following conditions:

(i) $\operatorname{Im}(\sigma) \subset W_{1}$.

(ii) $\operatorname{Im}\left(\sigma_{f}\right) \subset W_{f}$.

(iii) $d\left(\mathrm{pr}_{2} \circ \sigma\right)=d \gamma=0$, that is, $\gamma$ is closed. 
Using $\sigma$ we can define a vector field on $Q_{f}$ by

$$
X^{\sigma}(p)=T \operatorname{pr}\left(X\left(\sigma_{f}(p)\right)\right), \quad p \in Q_{f} .
$$

The above construction is illustrated in the following diagram

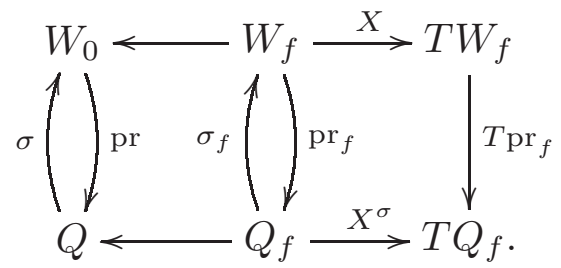

The relation between $T \sigma_{f}\left(X^{\sigma}\right)$ and $X$ is shown in the following theorem.

Proposition 4.2. Under the previous assumptions, the conditions

$$
d(D \circ \sigma)_{\mid Q_{f}}=0
$$

and

$$
\left(X-T \sigma_{f}\left(X^{\sigma}\right) \in \operatorname{ker}(\Omega)\right)_{\mid \operatorname{Im}\left(\sigma_{f}\right)}
$$

are equivalent.

Proof. The proof follows by similar arguments as in Proposition 4.1.

Definition 4.3. A section $\sigma$ of $T Q \oplus T^{*} Q, \sigma=(Z, \gamma)$, satisfying the following conditions:

(i) $\operatorname{Im}(\sigma) \subset W_{1}$.

(ii) $\operatorname{Im}\left(\sigma_{f}\right) \subset W_{f}$.

(iii) $d\left(\operatorname{pr}_{2} \circ \sigma\right)=d \gamma=0$.

(iv) $d(D \circ \sigma)_{\mid Q_{f}}=0$,

will be called a solution of the Hamilton-Jacobi problem for the Lagrangian $L$ in the Skinner-Rusk setting.

Remark 4.4. The last proposition says that $T \sigma_{f}\left(X^{\sigma}\right)$ is a vector field along $\operatorname{Im}\left(\sigma_{f}\right)$ which is also a solution of Eq. (3.2). So if we find an integral curve $c(t)$ of $X^{\sigma}$ on $Q_{f}$, then $\left(\sigma_{f} \circ c\right)(t)$ is an integral curve of a solution of $(3.2)$.

Remark 4.5. The natural question is if $X$ and $X^{\sigma}$ are $\sigma_{f}$-related in the singular case, as it happens in the standard Hamilton-Jacobi theory, see [17]. The answer is that, as we discussed later (Sec. 6), in some cases the vector fields are not necessarily $\sigma_{f}$-related.

\section{Comparison with the Hamiltonian and Lagrangian Settings}

In Sec. 4 we have developed a Hamilton-Jacobi theory in the Skinner-Rusk setting. The Skinner-Rusk formalism unifies Lagrangian and Hamiltonian formalisms, so we would like to relate the present Hamilton-Jacobi theory to the corresponding ones for the two formalisms (see [17]). 


\subsection{The Hamiltonian setting}

\subsubsection{The regular case}

If the Lagrangian, $L$, is regular, that is, $F L$ is a local diffeomorphism, then we can define locally a Hamiltonian function $h: T^{*} Q \rightarrow \mathbb{R}$ by $h=E_{L} \circ F L^{-1}$. Let us now assume that the Lagrangian is hyper-regular, that is, $F L$ is a global diffeomorphism and $h$ is globally defined. Denote by $X_{h}$ the corresponding Hamiltonian vector field

$$
i_{X_{h}} \Omega_{Q}=d h .
$$

Let $\gamma$ be a closed 1 -form on $Q$; then we can define a vector field on $Q$ by

$$
X^{\gamma}(p)=T \pi_{Q}\left(X_{h}(\gamma(p))\right) \quad \text { for all } p \in Q .
$$

Then we have the following Hamilton-Jacobi theorem.

Proposition 5.1. The vector fields $X$ and $X^{\gamma}$ are $\gamma$-related if and only if $d(h \circ \gamma)=0$.

Proof. For a proof see [1].

\subsubsection{The singular case}

Since we are considering an almost regular Lagrangian $L: T Q \rightarrow \mathbb{R}$, then we can apply the Dirac theory of constraints developed in Sec. 2.

We have to study the presymplectic system given by $\left(M_{1}, \Omega_{1}=j_{1}^{*} \Omega_{Q}, d h_{1}\right)$, where $j_{1}: M_{1} \rightarrow T^{*} Q$ is the inclusion and $h_{1}$ is defined implicitly by $h_{1} \circ F L=E_{L}$.

If we apply the Gotay-Nester-Hinds algorithm, we obtain a sequence of submanifolds

$$
\cdots M_{k} \hookrightarrow \cdots \hookrightarrow M_{2} \hookrightarrow M_{1} \hookrightarrow T^{*} Q ;
$$

assume that we obtain a final constraint submanifold, denoted by $M_{f}$. We also assume that $Q_{i}=\pi_{Q}\left(M_{i}\right)$ are submanifolds and that $\pi_{i}=\pi_{Q_{\mid M_{i}}}: M_{i} \rightarrow Q_{i}$ are submersions.

Remark 5.2. It is important to notice that the algorithm of Gotay-Nester-Hinds applied to the same Lagrangian considered in the Skinner-Rusk setting and in the corresponding Hamiltonian setting does not necessarily stop at the same level. For example, the Lagrangian given by $L\left(q^{1}, q^{2}, v^{1}, v^{2}\right)=v^{1} q^{2}$ produces the two presymplectic systems $\left(M_{1}, \Omega_{1}, d h_{1}\right)$ and $\left(W_{0}=T Q \oplus T^{*} Q, \Omega, d D\right)$. The first algorithm stabilizes in $k=1$, but the second one does in $k=2$.

Let $\gamma$ be a 1-form on $Q$ satisfying the following conditions:

(i) $\operatorname{Im}(\gamma) \subset M_{1}$.

(ii) $\operatorname{Im}\left(\gamma_{f}\right) \subset M_{f}$, where $\gamma_{f}$ denotes the restriction to $Q_{f}$ of $\gamma$.

(iii) $d \gamma=0$. 
Then, if $Y$ is a vector field on $M_{f}$ solving the equation $i_{Y} \Omega_{1}=d h_{1}$, we can construct the vector field $Y^{\gamma}$ on $Q_{f}$ given by

$$
Y^{\gamma}(p)=T \pi_{Q}\left(Y\left(\gamma_{f}(p)\right)\right), \quad \text { for each } p \in Q_{f}
$$

and obtain an analogous of Theorem 4.2 (notice that in this case we can ensure that the vector fields are $\gamma_{f}$-related, see [17] for the details).

Proposition 5.3. We have

$$
d\left(h_{1} \circ \gamma\right)_{\mid Q_{f}}=0 \Leftrightarrow Y \text { and } Y^{\gamma} \text { are } \gamma_{f} \text {-related } \text {. }
$$

Proof. Given $q \in Q_{f}$, we obtain

$$
\begin{aligned}
\left(i_{\left(Y(\gamma(q))-T_{q} \gamma_{f}\left(Y^{\gamma}(q)\right)\right)} \Omega_{1}\right) \circ T_{q} \gamma & =i_{Y(\gamma(q))} \Omega_{1} \circ T_{q} \gamma-i_{T_{q} \gamma_{f}\left(Y^{\gamma}(q)\right)} \Omega_{1} \circ T_{q} \gamma \\
& =d_{\gamma_{f}(q)} h_{1} \circ T_{q} \gamma=d_{q}\left(h_{1} \circ \gamma\right),
\end{aligned}
$$

where we have $T_{q} \gamma_{f}\left(Y^{\gamma}\right)=T_{q} \gamma\left(Y^{\gamma}\right)$ and

$$
\begin{aligned}
i_{T_{q} \gamma_{f}\left(Y^{\gamma}(q)\right)} \Omega_{1} \circ T_{q} \gamma(U) & =\Omega_{1}\left(T_{q} \gamma\left(Y^{\gamma}\right), T_{q} \gamma(U)\right) \\
& =\left(\gamma^{*} \Omega_{1}\right)\left(Y^{\gamma}(q), U\right)=-d \gamma\left(Y^{\gamma}(q), U\right)=0,
\end{aligned}
$$

for all $U \in T_{q} Q$.

The previous discussion can be applied to every point $q \in Q_{f}$; therefore, taking into account that $\Omega_{1}$ vanishes acting on two vertical tangent vectors, we can deduce the following

$$
Y-T \gamma_{f}\left(Y^{\gamma}\right) \in \operatorname{ker}\left(\Omega_{1}\right) \Leftrightarrow d\left(h_{1} \circ \gamma\right){ }_{\mid Q_{f}}=0 .
$$

As we did before, we will see that $Y$ and $Y^{\gamma}$ are $\gamma_{f}$-related.

Remember that for any point $p$ of $M_{1}$ we have a decomposition

$$
T_{p}\left(T^{*} Q\right)=T_{p} M_{1}+V_{p}\left(T^{*} Q\right)
$$

where $V\left(T^{*} Q\right)$ denotes as above the space of vertical tangent vectors on $p$.

Since $Y-T \gamma_{f}\left(Y^{\gamma}\right)$ is vertical at the points of $\operatorname{Im}\left(\gamma_{f}\right)$, given any $U \in V_{p}$, $p \in \operatorname{Im}\left(\gamma_{f}\right)$, then

$$
\Omega_{Q}\left(Y-T \gamma\left(Y^{\gamma}\right), U\right)=0
$$

Now, given $U \in T_{p} M_{1}$ we get

$$
\Omega_{Q}\left(Y-T \gamma_{f}\left(Y^{\gamma}\right), U\right)=\Omega_{1}\left(Y-T \gamma_{f}\left(Y^{\gamma}\right), U\right)=0,
$$

because $\left(Y-T \gamma_{f}\left(Y^{\gamma}\right)\right) \in \operatorname{ker}\left(\Omega_{1}\right)$, and hence $\Omega_{Q}\left(Y-T \gamma_{f}\left(Y^{\gamma}\right), Z\right)=0$ for any tangent vector $Z \in T_{p}\left(T^{*} Q\right)$ at any point of $\operatorname{Im}\left(\gamma_{f}\right)$. Since $\Omega_{Q}$ is non-degenerate, we deduce that $Y=T \gamma_{f}\left(Y^{\gamma}\right)$ along $\operatorname{Im}\left(\gamma_{f}\right)$.

Definition 5.4. A 1-form $\gamma$ satisfying the previous conditions will be called a solution of the Hamilton-Jacobi problem for $L$ in the Hamiltonian setting. 
We are now going to relate the Hamilton-Jacobi problem in the Skinner-Rusk setting and the corresponding one in the Hamiltonian setting. First, the following result gives the relation between $W_{i}$ and $M_{i}$, and also a relation between solutions of Eqs. (2.3) and (3.2).

Lemma 5.5. (i) If $X \in T_{p} W_{1}$ satisfies $i_{X} \Omega=d D$, then $X_{2}=T \operatorname{pr}_{2}(X) \in T_{\operatorname{pr}_{2}(p)}$ $M_{1}$ satisfies $i_{X_{2}} \Omega=d h_{1}$.

(ii) For each step $k$ of the constraint algorithms applied to the presymplectic systems $\left(M_{1}, \Omega_{1}, d h_{1}\right)$ and $\left(W_{0}=T Q \oplus T^{*} Q, \Omega, d D\right)$ we have

$$
\operatorname{pr}_{2}\left(W_{k}\right) \subset M_{k}
$$

and, if we denote the respective final constraint submanifolds by $W_{f}$ and $M_{f}$, then

$$
\operatorname{pr}_{2}\left(W_{f}\right)=M_{f}
$$

(iii) We have $\operatorname{pr}\left(W_{f}\right)=\pi_{Q}\left(M_{f}\right)=Q_{f}$.

Proof. (i) Recall that a vector $\xi \in T_{\left(q^{A}, v^{A}\right)} T Q, \xi=u^{A} \frac{\partial}{\partial q^{A}}+w^{A} \frac{\partial}{\partial v^{A}}$ satisfies $i_{\xi} \Omega_{L}=d E_{L}$ if and only if

$$
\begin{aligned}
\frac{\partial^{2} L}{\partial v^{A} \partial v^{B}}\left(v^{B}-u^{B}\right) & =0 \\
\frac{\partial^{2} L}{\partial v^{A} \partial v^{B}} u^{B}+\frac{\partial^{2} L}{\partial v^{A} \partial q^{B}} w^{B}-\frac{\partial L}{\partial q^{A}} & =\frac{\partial^{2} L}{\partial v^{B} \partial q^{A}}\left(v^{B}-u^{B}\right) .
\end{aligned}
$$

If $X \in T_{p} W_{1}$ verifies $i_{X} \Omega=d D$, then $X$ has the expression (3.4) and satisfies (3.5). So, it is clear that $X_{1}=T \operatorname{pr}_{1}(X)$ satisfies $i_{X_{1}} \Omega_{L}=d E_{L}$. Since $X$ is tangent to $W_{1}, X_{2}=T \operatorname{pr}_{2}(X)=T F L \circ T \operatorname{pr}_{1}(X)=T F L\left(X_{1}\right)$ and using Proposition 2.1 we can conclude that $i_{X_{2}} \Omega_{1}=d h_{1}$.

(ii) It will be proved by induction.

For $k=1$ we have that $\operatorname{pr}_{2}\left(W_{1}\right)=M_{1}$ since $W_{1}=\operatorname{graph}(F L)$.

Assume that $\operatorname{pr}_{2}\left(W_{k}\right) \subset M_{k}$. Then

$W_{k+1}=\left\{x \in W_{k}\right.$ such that there exists $X \in T_{x} W_{k}$ satisfying $\left.i_{X} \Omega=d D\right\}$,

$M_{k+1}=\left\{y \in M_{k}\right.$ such that there exists $Y \in T_{y} M_{k}$ satisfying $\left.i_{Y} \Omega_{1}=d h_{1}\right\}$.

If $x \in W_{k+1}$, then there exists $X \in T_{x} W_{k}$, satisfying $i_{X} \Omega=d D$. Since $\operatorname{pr}_{2}\left(W_{k}\right) \subset M_{k}, T \operatorname{pr}_{2}(X) \in T M_{k}$ and by (i) $i_{T p_{2}(X)} \Omega_{1}=d h_{1}$. Thus, we have proved that $\operatorname{pr}_{2}(x) \in M_{k+1}$ and that $\operatorname{pr}_{2}\left(W_{k}\right) \subset M_{k}$.

To prove that $\operatorname{pr}_{2}\left(W_{f}\right)=M_{f}$, take a solution $Y$ of Eq. (2.3) on $M_{f}$. Then we can construct a vector field $\xi$ on $P_{f}$ which is $F L_{f}$-related with $Y$, and using Theorem 2.2 we obtain a vector field $Y_{\xi}$ along the image of the section $\beta_{\xi}$ which satisfies $(2.1)$ and (2.2). We can construct the map

$$
\begin{aligned}
\bar{\beta}_{\xi}: \quad M_{f} & \rightarrow T Q \oplus T^{*} Q, \\
\left(q^{A}, p_{A}\right) & \rightarrow\left(\beta_{\xi}\left(q^{A}, p_{A}\right),\left(q^{A}, p_{A}\right)\right) .
\end{aligned}
$$


It is easy to see, that the vector field $T \bar{\beta}_{\xi}(Y)$ on $\operatorname{Im}\left(\bar{\beta}_{\xi}\right)$ is a solution of (3.2). By the maximality of the final constraint manifold $W_{f}$, we can conclude that $\operatorname{Im}\left(\bar{\beta}_{\xi}\right) \subset$ $W_{f}$, but $M_{f}=\operatorname{pr}_{2}\left(\operatorname{Im}\left(\bar{\beta}_{\xi}\right)\right) \subset \operatorname{pr}_{2}\left(W_{f}\right) \subset M_{f}$ and then the result follows.

(iii) It is a direct consequence of (ii) and the commutativity of diagram (3.1).

A solution of the Hamilton-Jacobi problem as stated in Sec. 4 is given by a section $\sigma$ of $T Q \oplus T^{*} Q$, so $\sigma=(Z, \gamma)$, where $Z$ and $\gamma$ are a vector field and a 1-form on $Q$, respectively.

We will see that $\gamma$ satisfies the Hamilton-Jacobi problem in the Hamiltonian sense.

From the fact that $\sigma$ is a solution of the Hamilton-Jacobi problem in the Skinner-Rusk setting, we deduce:

(i) Since $\operatorname{Im}(\sigma) \subset W_{1}$, then $\operatorname{Im}(\gamma)=\operatorname{pr}_{2}(\operatorname{Im}(\sigma)) \subset \operatorname{pr}_{2}\left(W_{1}\right)=M_{1}$.

(ii) Since $\operatorname{Im}\left(\sigma_{f}\right) \subset W_{f}$, then $\operatorname{Im}\left(\gamma_{f}\right)=\operatorname{pr}_{2}\left(\operatorname{Im}\left(\sigma_{f}\right)\right) \subset \operatorname{pr}_{2}\left(W_{f}\right)=M_{f}$.

(iii) Since $d\left(\operatorname{pr}_{2} \circ \sigma\right)=d \gamma=0$, then $\gamma$ is closed.

(iv) Since $\operatorname{Im}(\sigma) \subset W_{1}$, then $D \circ \sigma=h_{1} \circ \gamma$ and thus, using that $d(D \circ \sigma)_{\mid Q_{f}}=0$, we finally get $d\left(h_{1} \circ \gamma\right){ }_{\mid Q_{f}}=0$.

On the other hand, given a vector field $X$ on $W_{f}$ which is a solution of (3.2), we can obtain a solution of $(2.3)$ along $\operatorname{Im}\left(\gamma_{f}\right)$ by defining

$$
X_{2}\left(\gamma_{f}(p)\right)=T \operatorname{pr}_{2}\left(X\left(\sigma_{f}(p)\right)\right), \quad \text { for all } p \in Q_{f}
$$

Now, from Lemma 5.5 it follows that $X_{2}$ is a solution of (2.3).

As above we can construct the projected vector field on $Q_{f}$, by putting

$$
X_{2}^{\gamma}(p)=T \pi_{f}\left(X_{2}\left(\gamma_{f}(p)\right)\right), \quad \text { for all } p \in Q_{f} \text {. }
$$

Remark 5.6. By the commutativity of the diagram (3.1) we deduce that pr= $\pi_{Q} \circ \mathrm{pr}_{2}$, and in consequence we have

$$
X^{\sigma}(p)=T \operatorname{pr}\left(X\left(\sigma_{f}(p)\right)\right)=T \pi_{Q} \circ \operatorname{pr}_{2}\left(X\left(\sigma_{f}(p)\right)\right)=T \pi_{f}\left(X_{2}\left(\gamma_{f}(p)\right)\right)
$$

for all $p \in Q_{f}$, and so, $X^{\sigma}=X_{2}^{\gamma}$.

Summarizing the above discussion, we can conclude that it is possible to relate the Hamilton-Jacobi theory in the Skinner-Rusk setting to the Hamilton-Jacobi theory on $T^{*} Q$. In this case the vector fields $X_{2}$ and $X_{2}^{\gamma}$ are $\gamma_{f}$-related.

\subsection{The Lagrangian setting}

In this section we will relate the Hamilton-Jacobi theory developed in the SkinnerRusk setting with the corresponding one on the Lagrangian side. 


\subsubsection{The regular case}

If the Lagrangian $L$ is regular, then we have a symplectic system given by $\left(T Q, \Omega_{L}, E_{L}\right)$. Then there exists a unique solution $\xi$ of Eq. (2.1) which automatically satisfies the SODE condition.

Given $Z$ a vector field on $Q$ such that $Z^{*} \Omega_{L}=0$ we can define the following vector field on $Q$

$$
\xi^{Z}(p)=T \tau_{Q}(\xi(Z(p))) \quad \text { for all } p \in Q
$$

and obtain the following result.

Proposition 5.7. Under the previous conditions, the vector fields $\xi$ and $\xi^{Z}$ are $Z$-related if and only if $d\left(E_{L} \circ Z\right)=0$.

Proof. The proof is a consequence of Proposition 5.1.

\subsubsection{The singular case}

In this case, we will discuss the presymplectic system given by $\left(T Q, \Omega_{L}, d E_{L}\right)$. Applying the Gotay-Nester-Hinds algorithm we obtain a sequence of submanifolds

$$
\cdots P_{k} \hookrightarrow \cdots \hookrightarrow P_{2} \hookrightarrow P_{1}=T Q .
$$

We also assume that $Q_{i}=\tau_{Q}\left(P_{i}\right)$ are submanifolds and that $\tau_{i}=\tau_{Q_{\mid P_{i}}}: P_{i} \rightarrow Q_{i}$ are submersions, for any index $i$.

Remember that the algorithm of Gotay-Nester-Hinds applied to the presymplectic systems $\left(M_{1}, \Omega_{1}, d h_{1}\right)$ and $\left(T Q, \Omega_{L}, d E_{L}\right)$ stop at the same step, so we will denote the final constraint manifold of the system $\left(T Q, \Omega_{L}, d E_{L}\right)$ by $P_{f}$.

Let $Z$ be a vector field on $Q$ satisfying the following properties:

(i) $\operatorname{Im}\left(Z_{f}\right) \subset P_{f}$, where $Z_{f}$ denotes the restriction of $Z$ to $Q_{f}$.

(ii) $Z^{*} \Omega_{L}=0$.

Then, if $\xi$ is a vector field on $P_{f}$ solving the equation $i_{\xi} \Omega_{L}=d E_{L}$, we can construct the vector field $\xi^{Z}$ on $Q_{f}$ by

$$
\xi^{Z}(p)=T \tau_{Q}\left(\xi\left(Z_{f}(p)\right)\right), \quad \text { for all } p \in Q_{f} .
$$

Now, we can develop the corresponding Hamilton-Jacobi theory in the Lagrangian setting.

Proposition 5.8. Under the above hypothesis for $Z$ we have

$$
d\left(E_{L} \circ Z\right)_{\mid Q_{f}}=0 \Leftrightarrow\left(\xi-T Z_{f}\left(\xi^{Z}\right)\right) \in \operatorname{ker}\left(\Omega_{L}\right)_{\mid \operatorname{Im}\left(Z_{f}\right)} .
$$

Proof. " $\Rightarrow$ " Assume that $d\left(E_{L} \circ Z\right)_{\mid Q_{f}}=0$ holds, then we will prove that

$$
\left(i_{\left(\xi-T Z_{f}\left(\xi^{Z}\right)\right)} \Omega_{L}=0\right)_{\mid \operatorname{Im}\left(Z_{f}\right)} .
$$


For any $x \in \operatorname{Im}\left(Z_{f}\right)$ we have the decomposition $T_{x}(T Q)=T_{x} \operatorname{Im}(Z)+V_{x}(T Q)$, where $V(T Q)$ denotes the vertical bundle of the projection $\tau_{Q}: T Q \rightarrow Q$.

Since $\Omega_{L}$ vanishes acting on two elements of $V(T Q)$ and $\xi-T Z_{f}\left(\xi^{Z}\right)$ is vertical, we have

$$
\left(i_{\left(\xi-T Z_{f}\left(\xi^{Z}\right)\right)} \Omega\right)(V(T Q))=0 .
$$

Since $\xi$ is a solution along $\operatorname{Im}\left(Z_{f}\right)$, we obtain

$$
\left(i_{\xi(p)} \Omega_{L}\right) \circ T \sigma(p)=d_{Z(p)} E_{L} \circ T Z_{f}(p)=d_{p}\left(E_{L} \circ Z\right)
$$

for any $p \in Q_{f}$.

On the other hand, $\left(i_{T Z_{f}\left(\xi^{Z}(p)\right)} \Omega_{L}\right) \circ T Z(p)=0$, since for any $Y \in T_{p} Q$ we get

$$
\begin{aligned}
\left(i_{\left(\xi-T Z_{f}\left(\xi^{Z}\right)\right)} \Omega_{L}\right) \circ T Z(p)(Y) & =\Omega_{L}\left(T Z_{f}\left(\xi^{Z}(p)\right), T Z(Y)\right) \\
& =\left(Z^{*} \Omega_{L}\right)\left(\xi^{Z}, Y\right)=-d \gamma\left(\xi^{Z}, Y\right)=0
\end{aligned}
$$

and so we can conclude that

$$
\begin{aligned}
& \left(i_{\left(\xi-T Z_{f}\left(\xi^{Z}\right)\right)} \Omega_{L}\right)(T \operatorname{Im}(Z))=0 . \\
& \text { " } \Leftarrow \text { " Since } i_{\left(\xi-T Z_{f}\left(\xi^{Z}\right)\right)} \Omega_{L}=d\left(E_{L} \circ Z\right) \text {, if }\left(\xi-T Z_{f}\left(\xi^{Z}\right)\right) \in \operatorname{ker}\left(\Omega_{L}\right) \text {, then } \\
& d\left(E_{L} \circ Z\right)_{\mid Q_{f}}=0 \text {. }
\end{aligned}
$$

Definition 5.9. A vector field on $Q, Z$ satisfying the above conditions will be called a solution of the Hamilton-Jacobi problem for $L$ in the Lagrangian setting.

The vector fields $\xi$ and $\xi^{Z}$ are not necessarily related as the next example shows.

Example 5.10. Let $L: T \mathbb{R}^{2} \rightarrow \mathbb{R}$ be the Lagrangian given by

$$
L\left(q^{1}, q^{2}, v^{1}, v^{2}\right)=q^{1} v^{2}+q^{2} v^{1} .
$$

We have

$$
\begin{aligned}
F L\left(q^{1}, q^{2}, v^{1}, v^{2}\right) & =\left(q^{1}, q^{2}, q^{2}, q^{1}\right) \\
E_{L}\left(q^{1}, q^{2}, v^{1}, v^{2}\right) & =q^{1} v^{2}+q^{2} v^{1}-q^{1} v^{2}-q^{2} v^{1}=0 \\
\Omega_{L} & =0
\end{aligned}
$$

so every vector field $\xi$ on $T \mathbb{R}^{2}$ satifies

$$
i_{\xi} \Omega_{L}=d E_{L} .
$$

Therefore, the algorithm of Gotay-Nester-Hinds stabilizes at the first step, and $P_{f}=P_{1}=T Q$.

Moreover, every vector field $Z$ on $\mathbb{R}^{2}$ is a solution of the Hamilton-Jacobi problem, since $E_{L} \circ Z=0$ and $Z^{*} \Omega_{L}=0$.

Let $\xi$ be the solution satisfying the SODE condition given by

$$
\xi\left(q^{1}, q^{2}, v^{1}, v^{2}\right)=v^{1} \frac{\partial}{\partial q^{1}}+v^{2} \frac{\partial}{\partial q^{2}}+\frac{\partial}{\partial v^{1}}+\frac{\partial}{\partial v^{2}} .
$$


Let $Z$ be

$$
Z\left(q^{1}, q^{2}\right)=\frac{\partial}{\partial q^{1}}+\frac{\partial}{\partial q^{2}} .
$$

An easy computation shows that

$$
T Z\left(Z\left(q^{1}, q^{2}\right)\right)=\frac{\partial}{\partial q^{1}}+\frac{\partial}{\partial q^{2}},
$$

but

$$
\xi\left(Z\left(q^{1}, q^{2}\right)\right)=\frac{\partial}{\partial q^{1}}+\frac{\partial}{\partial q^{2}}+\frac{\partial}{\partial v^{1}}+\frac{\partial}{\partial v^{2}} \neq T Z\left(Z\left(q^{1}, q^{2}\right)\right) .
$$

Thus, the vector fields $\xi$ and $\xi^{Z}$ are not $Z$-related.

Next we will show that a solution of the Hamilton-Jacobi problem in the Skinner-Rusk formalism induces a solution of the Hamilton-Jacobi theory in the Lagrangian setting.

The following lemma is analogous to Lemma 5.5.

Lemma 5.11. (i) If $X \in T_{p} W_{1}$ satisfies $i_{X} \Omega=d D$, then $X_{1}=T \operatorname{pr}_{1}(X)$ satisfies $i_{X_{1}} \Omega_{L}=d E_{L}$ and the SODE condition (2.2).

(ii) For each step $k$ of the constraint algorithm applied to the presymplectic systems $\left(T Q, \Omega_{L}, E_{L}\right)$ and $\left(W_{0}=T Q \oplus T^{*} Q, \Omega, d D\right)$, we have

$$
\operatorname{pr}_{1}\left(W_{k}\right) \subset P_{k} .
$$

(iii) We have $\operatorname{pr}\left(W_{f}\right)=\tau_{Q}\left(P_{f}\right)$.

Proof. (i) and (ii) are proved using similar arguments to that in Lemma 5.5.

(iii) Since the following diagram

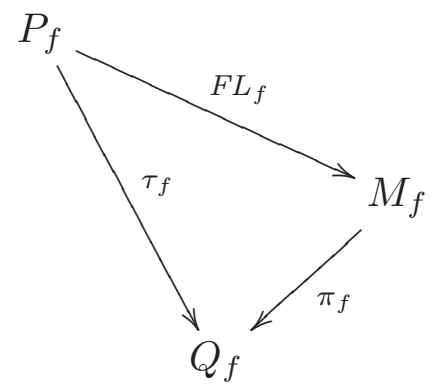

is commutative, and $F L_{f}$ is a surjective submersion, we deduce that $\pi_{Q}\left(M_{f}\right)=$ $\tau_{Q}\left(P_{f}\right)$. By Lemma 5.5(iii), we obtain $\pi_{Q}\left(M_{f}\right)=\operatorname{pr}\left(W_{f}\right)$, and the result follows. 
The situation can be summarized in the following commutative diagram

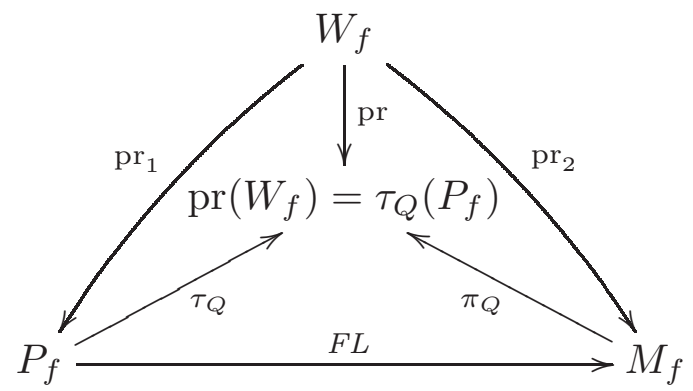

If $\sigma=(Z, \gamma)$ is a solution of the Hamilton-Jacobi problem, we deduce the following results:

(i) Since $\operatorname{Im}\left(\sigma_{f}\right) \subset W_{f}$, then $\operatorname{pr}_{1}\left(\operatorname{Im}\left(\sigma_{f}\right)\right) \subset \operatorname{pr}_{1}\left(W_{f}\right) \subset P_{f}$.

(ii) We have $Z^{*} \Omega_{L}=0$, since $Z^{*} \Omega_{L}=Z^{*}\left(d \theta_{L}\right)=d\left(Z^{*} \theta_{L}\right)=d(F L(Z))=d \gamma=0$.

(iii) Since $\operatorname{Im}(\sigma) \subset W_{1}$, then $D \circ \sigma(p)=E_{L} \circ Z(p)$ and, because $d(D \circ \sigma)_{\mid Q_{f}}=0$, then $d\left(E_{L} \circ Z\right)_{\mid Q_{f}}=0$.

Now, given a solution $X$ of $(3.2)$, we can obtain a solution of $(2.1)$ along $\operatorname{Im}\left(Z_{f}\right)$ using Lemma 5.11, and putting

$$
X_{1}\left(Z_{f}(p)\right)=T \operatorname{pr}_{1}(X(\sigma(p))), \quad \text { for all } p \in Q_{f} \text {. }
$$

We can also define the vector field on $Q_{f}$ given by

$$
X_{1}^{Z}(p)=T \tau_{Q}\left(X_{1}\left(Z_{f}(p)\right)\right) .
$$

The vector fields $X_{1}$ and $X_{1}^{Z}$ are not $Z_{f}$-related in general, as we have proved in Example 5.10.

Remark 5.12. By the commutativity of diagram (3.1) we have $\mathrm{pr}=\tau_{Q} \circ \mathrm{pr}_{1}$ and hence

$$
X^{\sigma}(p)=T \operatorname{pr}\left(X\left(\sigma_{f}(p)\right)\right)=T \tau_{Q} \circ \operatorname{pr}_{1}\left(X\left(Z_{f}(p)\right)\right)=T \tau_{Q}\left(X_{1}\left(Z_{f}(p)\right)\right)
$$

for all $p \in Q_{f}$, and so $X^{\sigma}=X_{1}^{Z}$.

Moreover, since $X_{1}$ satisfies the SODE condition, then

$$
X_{1}^{Z}(p)=T \tau_{Q}\left(X_{1}(Z(p))\right)=\tau_{T Q}\left(X_{1}(Z(p))\right)=Z(p)=Z_{f}(p),
$$

and we have

$$
X^{\sigma}=X_{1}^{Z}=X_{2}^{\gamma}=Z_{f}
$$

Note that this means that we only need to compute $X_{2}^{\gamma}$ to obtain $Z_{f}$. 


\section{Final Considerations}

In Sec. 5 we show that a solution of the Hamilton-Jacobi problem in the SkinnerRusk setting, $\sigma=(Z, \gamma)$, gives a solution of the Hamilton-Jacobi problem in the Lagrangian and Hamiltonian settings ( $Z$ and $\gamma$ respectively). A solution of Eq. (3.2) along $\operatorname{Im}(\sigma)$ can be also projected to solutions of (2.1) and (2.3) along $\operatorname{Im}(Z)$ and $\operatorname{Im}(\gamma)$, denoted respectively by $X_{1}$ and $X_{2}$.

If we take a vector field $X$ solution of Eq. (3.2) on $W_{f}$, using $\sigma$ we can compute $X^{\sigma}$. Now we can easily conclude that the vector fields $X$ and $X^{\sigma}$ are $\sigma_{f}$-related if and only if the corresponding vector fields $X_{1}$ and $X_{1}^{Z}$ are $Z_{f}$-related in the Lagrangian setting.

To illustrate the above results we revisited Example 5.10 in the Skinner-Rusk setting and apply the corresponding Hamilton-Jacobi theory.

Example 6.1. Consider the Lagrangian given in Example 5.10

$$
L\left(q^{1}, q^{2}, v^{1}, v^{2}\right)=q^{1} v^{2}+q^{2} v^{1} .
$$

Then, on $T \mathbb{R}^{2} \oplus T^{*} \mathbb{R}^{2}$ we have

$$
D\left(q^{1}, q^{2}, v^{1}, v^{2}, p_{1}, p_{2}\right)=v^{1} p_{1}+v^{2} p_{2}-v^{1} q^{2}-v^{2} q^{1},
$$

and hence

$$
\begin{aligned}
d D\left(q^{1}, q^{2}, v^{1}, v^{2}, p_{1}, p_{2}\right)= & -v^{2} d q^{1}-v^{1} d q^{2}+\left(p_{1}-q^{2}\right) d v^{1} \\
& +\left(p_{1}-q^{1}\right) d v^{2}+v^{1} d p_{1}+v^{2} d p_{2}
\end{aligned}
$$

Recall that we must compute

$$
\begin{aligned}
W_{1}= & \left\{\left(q^{A}, v^{A}, p_{A}\right) \text { such that there exists } X \in T_{\left(q^{A}, v^{A}, p_{A}\right)} T \mathbb{R}^{2} \oplus T^{*} \mathbb{R}^{2}\right. \\
& \text { satisfying } \left.i_{X} \Omega=d D\right\} .
\end{aligned}
$$

If

$$
X=a^{1} \frac{\partial}{\partial q^{1}}+a^{2} \frac{\partial}{\partial q^{2}}+b^{1} \frac{\partial}{\partial v^{1}}+b^{2} \frac{\partial}{\partial v^{2}}+c_{1} \frac{\partial}{\partial p_{1}}+c_{2} \frac{\partial}{\partial p_{2}}
$$

then

$$
i_{X} \Omega=-c_{1} d q^{1}-c_{2} d q^{2}+a^{1} d p_{1}+a^{2} d p_{2}
$$

and so

$$
a^{1}=v^{1}, \quad a^{2}=v^{2}, \quad c_{1}=v^{2}, \quad c_{2}=v^{1}, \quad p_{1}-q^{2}=0, \quad p_{2}-q^{1}=0
$$

must hold.

Therefore, $W_{1}=\left\{\left(q^{1}, q^{2}, v^{1}, v^{2}, q^{2}, q^{1}\right)\right.$ such that $\left.q^{A}, v^{A} \in \mathbb{R}\right\}=\operatorname{graph}(F L)$.

Next, we compute

$$
\begin{aligned}
W_{2}= & \left\{\left(q^{1}, q^{2}, v^{1}, v^{2}, q^{2}, q^{1}\right) \in W_{1}\right. \text { such that there exists } \\
& \left.X \in T_{\left(q^{1}, q^{2}, v^{1}, v^{2}, q^{2}, q^{1}\right)} W_{1} \text { satisfying } i_{X} \Omega=d D\right\} .
\end{aligned}
$$


If $X \in T W_{1}$ then $X$ can be locally expressed as

$$
\begin{aligned}
X= & a^{1} \frac{\partial}{\partial q^{1}}+a^{2} \frac{\partial}{\partial q^{2}}+b^{1} \frac{\partial}{\partial v^{1}}+b^{2} \frac{\partial}{\partial v^{2}} \\
& +\left(\frac{\partial^{2} L}{\partial v^{1} \partial q^{1}} a^{1}+\frac{\partial^{2} L}{\partial v^{1} \partial q^{2}} a^{2}+\frac{\partial^{2} L}{\partial v^{1} \partial v^{1}}+\frac{\partial^{2} L}{\partial v^{2} \partial v^{1}}\right) \frac{\partial}{\partial p_{1}} \\
& +\left(\frac{\partial^{2} L}{\partial v^{2} \partial q^{1}} a^{1}+\frac{\partial^{2} L}{\partial v^{2} \partial q^{2}} a^{2}+\frac{\partial^{2} L}{\partial v^{2} \partial v^{1}} b^{1}+\frac{\partial^{2} L}{\partial v^{2} \partial v^{2}} b^{2}\right) \frac{\partial}{\partial p_{2}} \\
= & a^{1} \frac{\partial}{\partial q^{1}}+a^{2} \frac{\partial}{\partial q^{2}}+b^{1} \frac{\partial}{\partial v^{1}}+b^{2} \frac{\partial}{\partial v^{2}}+a^{2} \frac{\partial}{\partial p_{1}}+a^{1} \frac{\partial}{\partial p_{2}} .
\end{aligned}
$$

Taking into account (6.4) and (6.5), for every point $\left(q^{1}, q^{2}, v^{1}, v^{2}, q^{2}, q^{1}\right)$ $\in W_{1}$ we obtain

$$
X=v^{1} \frac{\partial}{\partial q^{2}}+v^{2} \frac{\partial}{\partial q^{2}}+b^{1} \frac{\partial}{\partial v^{1}}+b^{2} \frac{\partial}{\partial v^{2}}+v^{2} \frac{\partial}{\partial p_{1}}+v^{1} \frac{\partial}{\partial p_{2}}
$$

for arbitrary $b^{1}, b^{2}$, and so $W_{2}=W_{1}$ and therefore the final constraint submanifold is $W_{1}$; consequently, $Q_{f}=Q$.

Now, a solution of the Hamilton-Jacobi problem in the Skinner-Rusk setting is given by $\sigma=(Z, \gamma)$ such that:

(i) $\operatorname{Im}(\sigma) \subset W_{1}$.

(ii) $\operatorname{Im}\left(\sigma_{f}\right) \subset W_{f}$.

(iii) $d\left(\mathrm{pr}_{2} \circ \sigma\right)=d \gamma=0$, that is, $\gamma$ is closed.

(iv) $d(D \circ \sigma)_{\mid Q_{f}}=0$.

It is easy to see that every pair given by a vector field $Z$ and its image by the Legendre transformation, that is $(Z, \gamma=F L(Z))$ is a solution of the problem. In fact, by construction $\operatorname{Im}(\sigma) \subset W_{1}$ and $D_{\mid W_{1}}=0 \Rightarrow D \circ \sigma=0$. Following the argument in Example 5.10 we can take $Z\left(q^{1}, q^{2}\right)=\frac{\partial}{\partial q^{1}}+\frac{\partial}{\partial q^{2}}$, and so

$$
\sigma\left(q^{1}, q^{2}\right)=\left(\frac{\partial}{\partial q^{1}}, \frac{\partial}{\partial q^{2}}, q^{2} d q^{1}+q^{1} d q^{2}\right) .
$$

If we consider the solution

$$
X\left(q^{1}, q^{2}, v^{1}, v^{2}\right)=v^{1} \frac{\partial}{\partial q^{1}}+v^{2} \frac{\partial}{\partial q^{2}}+\frac{\partial}{\partial v^{1}}+\frac{\partial}{\partial v^{2}}+v^{2} \frac{\partial}{\partial p_{1}}+v^{1} \frac{\partial}{\partial p_{2}},
$$

then

$$
X^{\sigma}\left(q^{1}, q^{2}\right)=\frac{\partial}{\partial q^{1}}+\frac{\partial}{\partial q^{2}}
$$

and

$$
T \sigma\left(X^{\sigma}\left(q^{1}, q^{2}\right)\right)=\frac{\partial}{\partial q^{1}}+\frac{\partial}{\partial q^{2}}+\frac{\partial}{\partial p_{1}}+\frac{\partial}{\partial p_{2}}
$$


A direct inspection shows that

$$
T \sigma\left(X^{\sigma}\left(q^{1}, q^{2}\right)\right) \neq X\left(\sigma\left(q^{1}, q^{2}\right)\right)=\frac{\partial}{\partial q^{1}}+\frac{\partial}{\partial q^{2}}+\frac{\partial}{\partial v^{1}}+\frac{\partial}{\partial v^{2}}+\frac{\partial}{\partial p_{1}}+\frac{\partial}{\partial p_{2}} .
$$

We can also obtain information of the Hamilton-Jacobi problem in the SkinnerRusk setting from a solution of the Hamilton-Jacobi problem in the Hamiltonian side.

If $\gamma$ is a solution of the Hamilton-Jacobi problem in the Hamiltonian setting and $Y$ a vector field on $M_{f}$ which is a solution of Eq. (2.3), then we can define $Y^{\gamma}$ as before.

We can also define a section $\tilde{\sigma}$ of $\operatorname{pr}_{f}: W_{f} \rightarrow Q_{f}$ given by $\tilde{\sigma}(p)=\left(Y^{\gamma}(p), \gamma(p)\right)$ for all $p \in Q_{f}$. An easy computation shows that $T \tilde{\sigma}\left(Y^{\gamma}\right)$ is a vector field along $\operatorname{Im}(\tilde{\sigma})$ which solves (3.2). Moreover if we find a vector field $Z$ on $Q$ such that $F L \circ Z=\gamma$ and $Z_{f}=Y^{\gamma}$, then the pair $(Z, \gamma)$ is a solution of the Hamilton-Jacobi problem in the Skinner-Rusk setting.

\section{Appendix A. The Gotay-Nester-Hinds Algorithm of Constraints}

In this section we will briefly review the constraint algorithm of constraints for presymplectic systems (see $[11,7]$ ).

Let $M_{1}$ be a manifold, $\Omega$ a presymplectic structure on $M_{1}$, i.e. $\Omega$ is a closed 2 -form, and $\alpha$ a 1-form on $M_{1}$. We will call $\left(M_{1}, \Omega, \alpha\right)$ a presymplectic system.

Gotay et al. developed an algorithm to find $N$, a submanifold of $M_{1}$ where we can solve the equation

$$
i_{X} \Omega=\alpha
$$

with $X$ tangent to $N$.

Equation (A.1) could not hold for every point of $M_{1}$, because $\alpha$ could not be in the range of $\Omega$. So it is necessary to introduce the following set

$$
M_{2}=\left\{p \in M_{1} \text { such that there exists } X \in T_{p} M_{1} \text { satisfying } i_{X} \Omega=\alpha\right\},
$$

and it is assumed that $M_{2}$ is a submanifold.

At the points of $M_{2}$ there exists solution to Eq. (A.1) but in an algebraic sense, that is, the solution could not be tangent to $M_{2}$. This forces a further restriction to

$$
M_{3}=\left\{p \in M_{2} \text { such that there exists } X \in T_{p} M_{2} \text { satisfying } i_{X} \Omega=\alpha\right\},
$$

which is also assumed to be a submanifold.

Proceeding as above, the algorithm will produce a sequence of submanifolds

$$
\cdots M_{3} \hookrightarrow^{j_{3}} M_{2} \hookrightarrow^{j_{2}} M_{1}
$$

where

$$
M_{l+1}=\left\{p \in M_{l} \text { such that there exists } X \in T_{p} M_{l} \text { satisfying } i_{X} \Omega=\alpha\right\},
$$

and $j_{l}$ denote the inclusions. 
There are three possibilities:

(i) There exists $k$ such that $M_{k}=\varnothing$.

(ii) There exists $k$ such that $M_{k}=M_{k+1}$.

(iii) The algorithm does not end.

In the second case the submanifold $M_{k}$ is called the final constraint submanifold and is denoted by $M_{f}$. By construction there exists a vector field on $M_{f}$ such as the solution of Eq. (A.1). The third case is only possible in the infinite-dimensional setting. In this case, the final constraint submanifold is defined by $M_{f}=\bigcap_{i=1} M_{i}$.

Note that the final constraint submanifold is maximal in the sense that if $R$ is submanifold of $M_{1}$ where there exists a tangent solution of Eq. (A.1), then $R \subset M_{f}$.

\section{Acknowledgments}

This work has been partially supported by MICINN (Spain) MTM2010-21186-C0201, the European project IRSES-project "Geomech-246981" and the ICMAT Severo Ochoa project SEV-2011-0087.

\section{References}

[1] R. Abraham and J. E. Marsden, Foundations of Mechanics, 2nd edn. (BenjaminCummings, Reading, MA, 1978).

[2] V. I. Arnold, Mathematical Methods of Classical Mechanics, 2nd edn., Graduate Texts in Mathematics, Vol. 60 (Springer-Verlag, New York, 1989).

[3] J. F. Cariñena, X. Gracia, G. Marmo, E. Martínez, M. Muñoz-Lecanda and N. Román-Roy, Geometric Hamilton-Jacobi theory, Int. J. Geom. Meth. Mod. Phys. 3(7) (2006) 1417-1458.

[4] J. F. Cariñena, X. Gracia, G. Marmo, E. Martínez, M. Muñoz-Lecanda and N. Román-Roy, Geometric Hamilton-Jacobi theory for nonholonomic dynamical systems, Int. J. Geom. Meth. Mod. Phys. 7(3) (2010) 431-454.

[5] J. F. Cariñena, X. Gracia, G. Marmo, E. Martínez, M. Muñoz-Lecanda and N. Román-Roy, Hamilton-Jacobi theory and the evolution operator, preprint (2009), arXiv: 0907.1039.

[6] P. A. M. Dirac, Lectures on Quantum Mechanics [Second printing of the 1964 original], Belfer Graduate School of Science Monographs Series, Vol. 2 (Academic Press, New York, 1967).

[7] M. J. Gotay, Presymplectic manifolds, geometric constraint theory and the DiracBergmann theory of constraints, Ph.D. thesis, University of Maryland (1979).

[8] M. J. Gotay and J. M. Nester, Presymplectic Lagrangian systems. I. The constraint algorithm and the equivalence theorem, Ann. Inst. H. Poincaré Sec. A (NS) 30(2) (1979) 129-142.

[9] M. J. Gotay and J. M. Nester, Presymplectic Lagrangian systems. II. The secondorder equation problem, Ann. Inst. H. Poincaré Sec. A (NS) 32(1) (1980) 1-13.

[10] M. J. Gotay and J. M. Nester, Generalized constraint algorithm and special presymplectic manifolds, in Geometric Methods in Mathematical Physics, Lecture Notes in Mathematics, Vol. 775 (Springer, Berlin, 1980), p. 78,104.

[11] M. J. Gotay, J. M. Nester and G. Hinds, Presymplectic manifolds and the DiracBergmann theory of constraints, J. Math. Phys. 19(11) (1978) 2388-2399. 
[12] M. Leok, T. Ohsawa and D. Sosa, Hamilton-Jacobi theory for Degenerate lagrangian systems with holonomic and nonholonomic constraints, J. Math. Phys. 53(7) (2012) 072905, 29 pp.

[13] M. de León, D. Iglesias-Ponte and D. Martín de Diego, Towards a Hamilton-Jacobi theory for nonholonomic mechanical systems, J. Phys. A, Math. Theor. 41(1) (2008) $14 \mathrm{pp}$.

[14] M. de León, J. C. Marrero and D. Martín de Diego, A geometric Hamilton-Jacobi theory for classical field theories, in Variations, Geometry and Physics (Nova Science Publishers, New York, 2009), pp. 129-140.

[15] M. de León, J. C. Marrero and D. Martín de Diego, Linear almost Poisson structures and Hamilton-Jacobi equation. Applications to nonholonomic mechanics, J. Geom. Mech. 2(2) (2010) 159-198.

[16] M. de León and D. Martín de Diego, A constraint algorithm for singular Lagrangians subjected to nonholonomic constraints, J. Math. Phys. 38(6) (1997) 3055-3062.

[17] M. de León, J. C. Marrero, D. Martín de Diego and M. Vaquero, A Hamilton-Jacobi theory for singular Lagrangian systems, preprint (2012), arXiv: 1204.6217.

[18] M. de León, D. Martín de Diego and M. Vaquero, A geometric Hamilton-Jacobi theory for multisymplectic field theories, in preparation.

[19] M. de León and P. R. Rodrigues, Methods of Differential Geometry in Analytical Mechanics, North-Holland Mathematics Studies, Vol. 158 (North-Holland Publishing, Amsterdam, 1989).

[20] G. Longhi, D. Dominici, J. Gomis and J. M. Pons, The Hamilton-Jacobi formalism for systems with constraints, Relativistic Action at a Distance: Classical and Quantum Aspects, Lecture Notes in Physics, Vol. 162 (Springer, Berlin, 1982), pp. 165-189.

[21] G. Longhi, D. Dominici, J. Gomis and J. M. Pons, Hamilton-Jacobi theory for constrained systems, J. Math. Phys. 25(8) (1984) 2439-2452.

[22] K. D. Rothe and F. G. Scholtz, On the Hamilton-Jacobi equation for second-class constrained systems, Ann. Physics 308(2) (2003) 639-651.

[23] H. Rund, The Hamilton-Jacobi Theory in the Calculus of Variations (Hazell, Watson and Viney, Aylesbury, Buckinghamshire, UK, 1966).

[24] R. Skinner and R. Rusk, Generalized Hamiltonian dynamics. I. Formulation on $T Q \oplus$ TQ, J. Math. Phys. 24(11) (1983) 2589-2594.

[25] R. Skinner and R. Rusk, Generalized Hamiltonian dynamics. II. Gauge transformations, J. Math. Phys. 24(11) (1983) 2595-2601.

[26] L. Vitagliano, The Hamilton-Jacobi formalism for higher-order field theories, Int. J. Geom. Math. Mod. Phys. 7 (2010) 1413-1436.

[27] H. Yoshimura and J. E. Marsden, Dirac structures in Lagrangian mechanics. I. Implicit Lagrangian systems, J. Geom. Phys. 57(1) (2006) 133-156. 


\section{Conclusions and Future Work}

We end this memory analyzing some of the results.

\subsection{Conclusions}

The extension of the Hamilton-Jacobi theory to more general geometrical settings has proved to be very fruitful along this dissertation. The Hamilton-Jacobi theory was extended to the following frameworks:

1. Reduction theory (actually Atiyah Bundles $T^{\star} Q / G$ ).

2. Dual bundles of Lie algebroids (linear Piosson structures).

3. Almost-Poisson structures.

4. Degenerate lagrangians.

The reduction theory for the Hamiton-Jacobi equation introduced in [22] should be applied to other examples. The importance of symmetry in mechanical systems is clearly everywhere. Many systems can be studied under this setting. We show that we were able to understand the geometry of the problem through the notion of lagrangian submanifolds. Although the geometry of the problem is clear now, it is far from being exploited. We present in the next section some future work in this regard.

Among all the results presented here, the work developed in [29] seems to be especially well suited for future research. One of the short term objectives is to work on all the possible numerical applications that it provides. Moreover, these numerical applications where already tested, but in a very restrictive framework, dual of Lie algebras with generating functions of type 1. All this can be shown by the big amount of work related to this approaches, [7, 11, 27, 33, 34, 43, 51]. For a survey of this previous work we refer to [44]. In that paper, it was asked how to generate the identity from a generating function, emulating the classical non free generating function. With our geometric approach, we developed an easy way to do that. To generate the identity is also very important because the symplectic flow is regarded as a perturbation to certain order of the lagrangian submanifold that gives the identity, through the image of the corresponding generating function. To develop Poisson integrators is very important, and it is justified by the main role played 
by symplectic integrators in the symplectic context. We discovered that similar ideas were discussed by Ge ([30]) and Scovel and Weinstein [52], but they did not deepen in this issue. Nonetheless, we still have to refine our numerical methods and apply them to more situations. On the other hand, the geometric setting seems to be very complete.

The Hamilton-Jacobi theory for almost-Poisson setting seems to be very clear now. We gave a neat explanation of the geometry and treated some examples. One of the main ideas of this approach was to develop a theory of complete solution for non-holonomic mechanical systems. However, it turns out, that such a theory seems not posible from this viewpoint. The problem is that although one can look for a lagrangian submanifold tangent to the flow, and obtain a "simple" solution of the Hamilton-Jacobi theory that allows the reduction of the dynamics, the flow of a non-holonomic system seems not to be a lagrangian submanifold in the product manifold $\mathbb{R}^{2} \times P \ominus P$, contrary to the symplectic case. More explanations about the failure of this approach were also discussed in [5].

At the end of this dissertation we developed a Hamilton-Jacobi theory for singular lagrangians. The aim of that theory was a two-fold. On one hand, the development of a Hamilton-Jacobi theory for singular lagrangians in mechanics is interesting by itself. On the other hand, it served as a test to see how to develop a Hamilton-Jacobi theory for classical field theory. This is also a field of study and some works are getting done in this direction. We analyzed some examples and showed how this theory works.

\subsection{Future Work}

- Relate our theory of generating functions with symmetry to the theory in [45, 46]. The theory developed there relies on generating function, so it seems that our theory should be the natural framework to deal with this kind of theories. Connections with the Poincaré generating function would be also very interesting.

- Extend our theory of reduction to general symmetries. Although quite useful our setting only deals at this moment with cotangent lifts of symmetries, to develop an analogous theory for any kind of symmetries should provide means to integrate more general systems. The results in [28] could be of some help in this regard.

- Implementation and improvement of numerical methods based on [29]. As we said before, this seems to be the first time that the whole geometric picture of the HamiltonJacobi is clear in this setting, after the attempt made in [33]. The application of the now well-known geometric results to concrete situations seems to be the most urgent work to be done. We also want to stress that the numerical methods that we present here are very general and conserve the geometry very well, but they are not very efficient from the computational viewpoint. Our aim in this paper was to show how to use our results, more that giving optimized numerical methods. Nonetheless, there is still a lot of room to improve these methods, see the last section of [29]. Those improvements can be applied in a straightforward fashion to our setting. Morevoer, the development of Ruth type integrators should be possible and clear now. 
- Truncation of infinite dimensional Hamiltonian systems. Hamiltonian truncation conserving the geometry, as much as possible, is very important to approximate infinite dimensional systems. This approximation can be used to understand the qualitative behavior of the infinite dimensional Hamiltonian dynamical system or to build numerical integrators. For instance, I would like to highlight the truncation in [52], which is a first instance about how this truncation should be carried out. The main ideas come from symplectic groupoids and Lie algebroids, as we explained in Chapter 3. The dual of a Lie algebroid has a natural linear Poisson structure that should be appropriate to develop the mentioned approximation.

- Study of the work by Moser and Veselov ([47]) to better understand completely integrable systems. The framework used by the authors was described in a groupoidalgebroid setting by A. Weinstein in [56], which is directly related to my previous work. Assuming that we are dealing with an integrable Poisson structure, which as I said is the case for most interesting cases in classical mechanics, uncovering relations between the completely integrable Poisson systems and the symplectic groupoids integrating it, would be geometrically very interesting.

- Extension of results based on generating functions to the Poisson setting. There are results in symplectic geometry strongly based on the idea of generating functions. With our theory of generating functions for Poisson manifolds, new extensions should be now applicable to Poisson geometry. For example, the results in Appendix 9 in [3] about fixed points of symplectomorphisms of the annulus. Another very interesting topic is the extension of Lindtstetd's method for eliminating fast variables. This method relies on the contruction of a series of canonical transformations through generating functions. To see to what extent the "wild" Poisson world admits these constructions is very interesintg from the geometric viewpoint.

Here I included some of most immediate research projects that I plan to develop. At the end of $[22,29]$ there are more interesting directions of research. 


\section{Conclusiones y Trabajo Futuro}

Finalizamos esta memoria analizamondo algunos de los resultados obtenidos y futuras lineas de trabajo.

\subsection{Conclusiones}

La extensión de la teoría de Hamilton-Jacobi a geometrías más generales ha demostrado ser muy útil a lo largo de esta memoria. La teoría de Hamilton-jacobi se ha extendido a los siguientes marcos geométricos:

1. Reducción (que se corresponde con los fibrados de Atiya $T^{\star} Q / G$ ).

2. Fibrados duales de algebroides de Lie (estructuras de Poisson lineales).

3. Estructuras casi-Poisson.

4. Lagrangianos degenerados.

Es destacable que la teoría de reducción para la ecuación de Hamilton-Jacobi que fue introducida en el trabajo [22], todavía puede ser utilizada en muchas más situaciones. La importancia de las simetrás en los sistemas mecánicos es clara y bien conocida desde los principios de la mecánica clásica, pues muchos sistemas se pueden estudiar bajo este marco. En este memoria hemos mostrados una comprensión completa de la geometrá subyacente del proceso de reducción y reconstrucción de la ecuación de Hamilton-Jacobi mediante el uso de subvariedades lagrangianas. Aunque la geometrá del problema esta ahora completamente desarrollada, todavía queda mucho margen para desarrollar nuevas aplicaciones. Listamos más abajo algunas de las lineas de trabajo futuro que planteamos seguir.

De entre los resultados presentados aquí, los obtenidos en el trabajo [29] parecen especialmente importantes para el futuro próximo. Uno de los objetivos a corto plazo es trabajar en las aplicaciones numéricas que dichos resultados proveen. Más aún, estas aplicaciones numéricas ya han sido testadas en trabajos previos, pero únicamente en ciertas situaciones muy restrictivas, duales de álgebras de Lie usando funciones generatrices del llamado tipo I. Una prueba de la importancia de estos resultados es la cantidad de trabajo que se ha llevado a cabo referente a estos métodos, [7, 11, 27, 33, 34, 43, 51]. Para un 
resumen del trabajo previo en este sentido referimos al lector a [44]. En dicho artículo, uno de los problemas abiertos planteados es cómo generar la transformación identidad desde las funciones generatrices, emulando la situación clásica. Con nuestro enfoque geométrico hemos conseguido responder a esa pregunta de manera afirmativa. Generar la identidad es una cuestión muy importante porque los métodos simplécticos presentados aquí se interpretan como una perturbación de la subvariedad lagrangiana que produce la transformación identidad, a través de la correspondiente función generatriz. Desarrollar integradores de Poisson se presenta como una tarea muy importante, que está sobradamente justificada por el papel principal que juegan los integradores simplécticos en la geometría simpléctica. Durante la elaboración de esta memoria descubrimos que ideas similares fueron discutidas por Ge ([30]) y Scovel y Weinstein [52], pero sin llegar a profundizar en estos temas. La descripción geométrica que permite la elaboración de estos integradores está ahora totalmente completa. Sin embargo, consideramos que todavía tenemos que refinar nuestros métods numéricos y aplicarlos a otras situaciones.

Una teorá de Hamilton-Jacobi para variedades de Poisson está finalmente descrita. Damos una explicación clara de la geometría correspondiente y tratamos algunos ejemplos. Una de las ideas principales de este punto de vista era la de desarrollar un concepto de soluciones completas para sistemas no-holónomos. Desafortunadamente, hemos comprobado que tal teoría no puede ser alcanzada desde este punto de vista. El problema reside en que aunque uno puede buscar subvariedades lagrangianas tangentes a un campo de vectores hamiltoniano, y de esta manera obtener soluciones "simples" de la ecuación de Hamilton-Jacobi que permiten una reducción de la dinámica, el flujo de un sistema noholónomo no es una subvariedad lagrangian en el producto $\mathbb{R}^{2} \times P \ominus P$, contrariamente al caso simpléctico. Más detalles sobre esta carencia se pueden encontrar en [5], trabajo que apareció simultaneamente a nuestros resultados.

En la parte final de esta memoria hemos desarrollado una teoría de Hamilton-Jacobi para lagrangianos singulares. La intención de dicha teoría era doble, por un lado, desarrollar una teoría de Hamilton-Jacobi para lagrangianos singulares, interesante por si sola, para sistemas gobernados por la mecánica clśica. Por otro, usar dicha teoría como modelo para la correspondiente extensión a al teoría clásica de campos. En esta última dirección se están llevando a cabo más trabajos que aparecerán próximamente en revistas de conocido prestigio internacional.

\subsection{Trabajo Futuro}

Listamos algunas de las lineas a seguir en el futuro próximo.

- Relacionar nuestra teoría de funciones generatrices con simetría con los resultados presentados en [45, 46]. La teoría presentada en dichos trabajos se basa en funciones generatrices y su invarianza bajo ciertas simetrías, por lo que parece que nuestra teoría es el ámbito natural para entender y mejorar este tipo de resultados. Conexiones con las funciones generatrices de Poincaré serían otro tema a tratar. 
- Extender nuestra teoría de reducción a simetrías más generales. Aunque muy útil, nuestra teoría solo contempla, en este momento, simetrías levantadas a fibrados cotangentes. Desarrollar una teoría análoga para otros tipos de simetrás proporcionaría métodos para integrar sistemas más generales. Los resultados de [28] deberían ayudar en este sentido.

- Implementación y mejora de los métodos numéricos basándose en [29]. Tal y como hemos dichos anteriormente, esta parece ser la primera vez que el esquema geométrico está claro, después de los intentos hechos en [33]. La aplicación de estos resultados a situaciones concretas parece ser uno de los principales trabajos a llevar a cabo. También es destacable que los métodos numéricos aquí presentados son muy generales y conservan la geometría de manera excepcional, pero no son muy eficientes desde el punto de vista computacional. Nuestra intención en este trabajo es mostrar como usar estos resultados, más que construir métodos optimizados. Sin embargo, hay todavía muchas mejoras que se pueden incorporar a estos métodos de manera muy directa, como mostramos en la parte final de [29]. Más aún, el desarrollo de integradores tipo Ruth en geometría de Poisson es posible ahora, gracias a nuestros resultados teóricos.

- Truncación de sistemas hamiltonianos en dimension infinita. Truncar un sistema hamiltoniano conservando la geometría al máximo es muy importante a la hora de aproximar sistemas de dimensión infinita. Estas aproximaciones se pueden usar para entender cualitativamente el comportamiento de sistemas hamiltonianos de dimensión infinita y para construir métodos numéricos. Por ejemplo, nos gustaría señalar la truncación llevada a cabo en [52], que es un claro ejemplo de como llevar a cabo este proceso. La idea principal es el uso de grupoides simplécticos y algebroides de Lie, como explicamos en el Capítulo 3. El fibrado dual de un algebroide de Lie tiene una estructura de Poisson lineal que parece ser la apropiada para desarrollar las aproximaciones mencionadas.

- Estudio de los resultados de Moser y Veselov ([47]). El esquema desarrollado por los autores fue formalizado usando grupoides y algebroides por A. Weinstein en [56], que está directamente relacionado con nuestros resultados anteriores. Si asumimos que estamos tratando con una estructura de Poisson integrable, que como hemos dicho previamente se corresponde con los casos más importantes en mecánica clásica, descubrir relaciones entre sistemas Poisson completamente integrables y el grupoide simpléctico que las integra será geométricamente muy interesante.

- Extensión de resultados basados en funciones generatrices al caso Poisson. Hay muchos resultados en geometría simpléctica basados en la idea de función generatriz. Con nuestra teoría de funciones generatrices para variedades de Poisson, nuevas extensiones de esos resultados podrían aplicarse ahora a la geometría de Poisson. Por ejemplo, los resultados en el apéndice 9 de [3] sobre puntos fijos de simplectomorfismos del annulus. Otra tema muy interesante sería la extensión del método de Lindstetd para eleminar las "fast vaariables". Este métodos se basa en la construcción de una 
serie de transformaciones canónicas usando funciones generatrices. Ver hasta que punto la geometría de Poisson admite estas construcciones es muy interesnate desde el punto de vista geométrico.

Aquí hemos incluido algunos de las lineas de trabajo más inmediatas que planeamos desarrollar. Al final de los trabajos [22, 29] se han presentado otras direcciones a seguir. 